\title{
A Numerical Model of an Active Magnetic Regenerator Refrigeration System
}

\author{
by
}

Kurt Engelbrecht

A thesis submitted in partial fulfillment of the requirements for the degree of

Master of Science

(Mechanical Engineering)

at the

UNIVERSITY OF WISCONSIN-MADISON

2004 

Approved by

Professor Gregory F. Nellis Date 



\begin{abstract}
$\underline{\text { Abstract }}$
Active magnetic regenerator refrigeration (AMRR) systems are an environmentally attractive space cooling and refrigeration alternative that do not use a fluorocarbon working fluid. Two recent developments have made AMRRs appear possible to implement in the near-term. A rotary regenerator bed utilizing practical and affordable permanent magnets has been demonstrated to achieve acceptable COP. Concurrently, families of magnetocaloric material alloys with adjustable Curie temperatures have been developed. Using these materials it is possible to construct a layered regenerator bed that can achieve a high magnetocaloric effect across its entire operating range, resulting in an improved COP.
\end{abstract}

There is currently no tool capable of predicting the performance of a layered AMRR. This project provides a numerical model that predicts the practical limits of these systems for use in space conditioning and refrigeration applications. The model treats the regenerator bed as a one dimensional matrix of magnetic material with a spatial variation in Curie temperature and therefore magnetic properties. The matrix is subjected to a spatially and temporally varying magnetic field and fluid mass flow. The variation of these forcing functions is based on the implementation of a rotating, multiple bed configuration. The numerical model is solved using a fully implicit (in time and space) discretization of the governing energy equations. The nonlinear aspects of the governing equations (e.g., fluid and magnetic property variations) are handled using a relaxation technique.

The model is used to optimize AMRR applications by varying model inputs such as matrix material, fluid mass flow rate, working fluid, reservoir temperatures, and the variation of the Curie temperature across the bed. The preliminary model has been verified qualitatively using simple cycle parameters and constant property materials and quantitatively by comparing the results with prior solutions to the regenerator governing equations in the limits of constant properties and no magnetocaloric effect. A second goal of this project is to create a cost estimate for a future project that will design, build, and test a prototype AMRR to be used to verify the numerical model. 



\section{Acknowledgements}

First and foremost, I would like to thank my advisors Professor Greg Nellis and Professor Sanford Klein for their guidance and help throughout this project. They have both helped me achieve more than I ever thought possible over the past 15 months.

I would like to thank my father for encouraging me to continue my education and my mother for her support. Thanks to my sisters Sarah, Claire, and Rosie who have always been there for me whenever I needed to talk, share stories, or just have a good laugh. I would also like to thank my grandparents for their continued love and support.

It has been a pleasure to work in the SEL due in large part to the company and tomfoolery of my fellow students. I would like to thank the following students in particular for breaking up the monotony of my studies and keeping my outlook on work positive: Seth Potratz, Mike Cheadle, Widalys DeSoto, Michael Frank, Kylie Fredrickson, Gayathri Vijayakumar, Scott Schuetter, Cory Hughes, Ryan Taylor, Holly Burnett, and Brandon Lachner.

I would like to thank Professor Doug Reindl, Professor Tim Shedd, Carl Zimm, and Alexander Sternberg for their technical assistance.

Finally, I would like to thank the Air-Conditioning and Refrigeration Institute for their financial support. 



\section{Table of Contents}

\section{Chapter 1 Introduction ........................................................................... 1}

$1.1 \quad$ The Magnetocaloric Effect.......................................................

$1.2 \quad$ Magnetic Refrigeration .........................................................

1.3 Active Magnetic Regenerative Refrigeration Cycle .................8

$1.4 \quad$ Tunable Magnetocaloric Materials ………………………….....18

$1.5 \quad$ Project Objectives ………………………………………....... 21

\section{Chapter 2 - Numerical Model of Regenerator Bed ................. 24}

2.1 Governing Equations..............................................................24

$2.2 \quad$ Numerical Solution Algorithm..................................................38

2.3 Model Verification ................................................................. 40

2.4 Material Properties ………………………………………........4

2.4.1 Heat Transfer Fluids ..........................................................43

2.4.2 Magnetocaloric Materials....................................................4

2.5 Regenerator Correlations............................................................

2.5.1 Pressure Drop ………………………………………….....

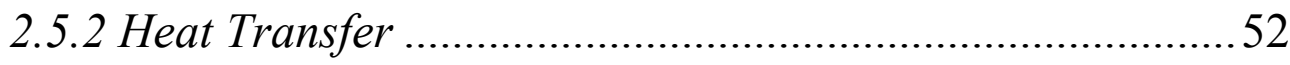

2.5.3 Axial Conduction ………………………………………...... 53

2.6 Correction for Internal Temperature Gradients...........................57

2.7 Correction for Entrained Heat Capacity ........................................60 
2.8 Validation of the Numerical Model...........................................61

\section{Chapter 3 - Passive Regenerator Test Results..................... 72}

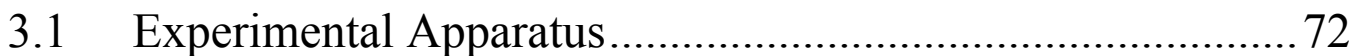

3.2 Comparison of Model Predictions with Data.........................74

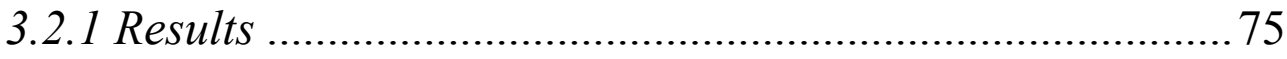

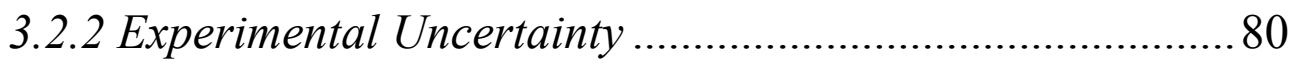

\section{Chapter 4 - System Modeling................................................... 82}

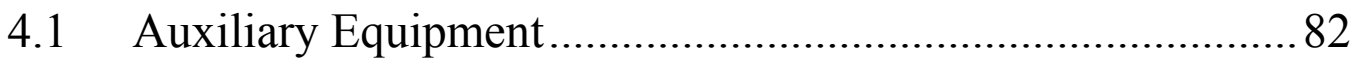

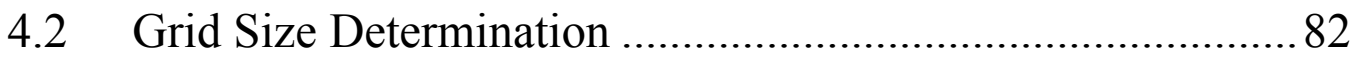

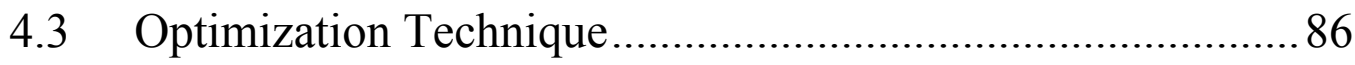

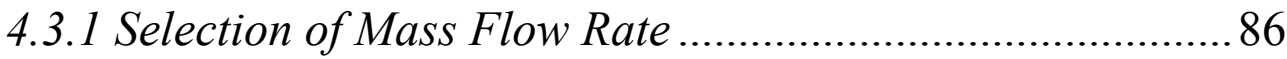

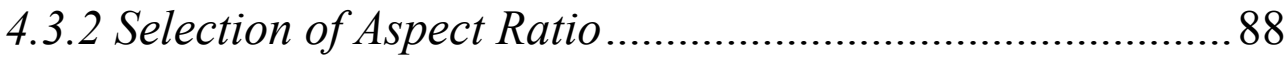

4.4 Space Conditioning Application ........................................ 90

4.4.1 Heat Exchanger Modeling ................................................90

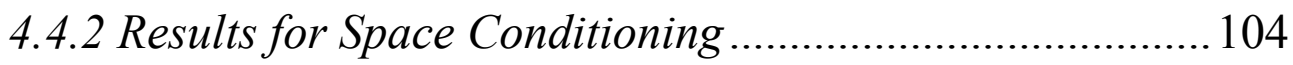

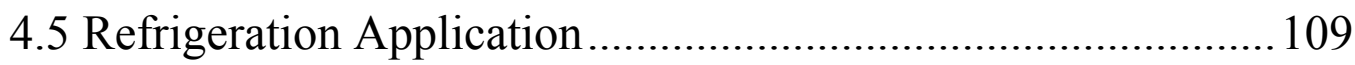

4.5.1 Heat Exchanger Modeling ................................................. 109

4.5.2 Results for Refrigeration Application ...............................109

\section{Chapter 5 - Parametric Studies .............................................. 116}

5.1 Advanced Regenerator Results .......................................... 116 
5.1.1 Advanced Regenerator Bed Correlations and Material Properties 116

5.1.2 Optimum Porosity for the Advanced Regnerator 120

5.1.3 Advanced Regenerator Results 122

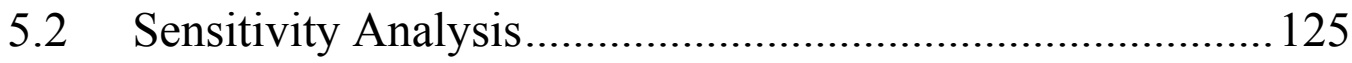

5.3 Identification of Risk Areas .............................................. 129

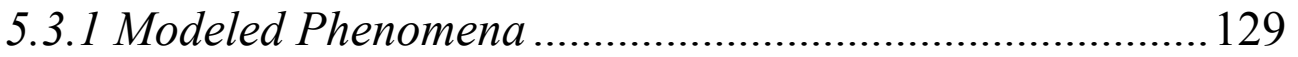

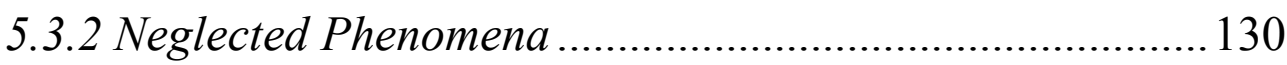

Chapter 6-Conclusions ....................................................... 133

References................................................................................ 138

Appendix A...................................................................... 142 


\section{List of Figures}

1.1 Analogy between magnetic and mechanical work transfer ........................................2

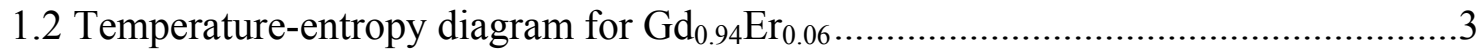

1.3 Adiabatic temperature change with magnetization for $\mathrm{Gd}_{0.94} \mathrm{Er}_{0.06} \ldots \ldots \ldots \ldots \ldots \ldots \ldots \ldots \ldots . . .4$

1.4 Temperature-entropy diagram for a one-shot refrigeration cycle ..............................6

1.5 Active magnetic regenerative refrigeration cycle .................................................11

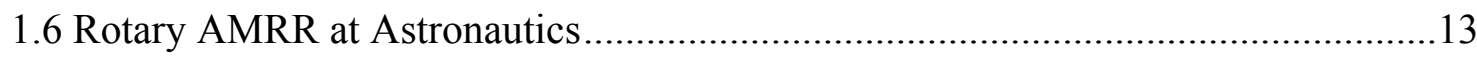

1.7 Type A bed entering the magnetic field and being magnetized from hot-to-

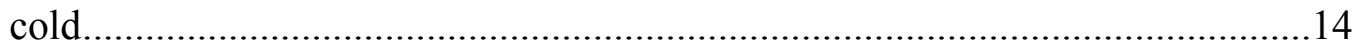

1.8 Type-A bed fully in the magnetic field and subjected to flow from the cold end to the hot end. Note the Type-B bed entering the magnetic field and being magnetized from its cold end to its hot end............................................... 15

1.9 Type A bed leaving the magnetic field ..................................................................16

1.10 Type A Bed experiencing hot-to-cold flow ………..........................................17

1.11 Magnetocaloric effect for pure gadolinium (source: Ames Laboratory) .................19

1.12 Magnetic entropy change for the $\mathrm{LaFe}_{11.5} \mathrm{Si}_{1.5} \mathrm{H}_{\mathrm{y}}$ for $\mathrm{y}=1.5,1.3,0.6$, and 0.3

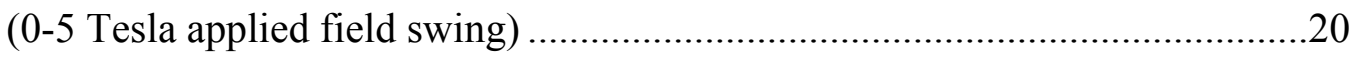

2.1 Conceptual drawing of a 1D AMR model showing the important parameters.........25

2.2 Differential segment of entrained fluid with energy terms indicated ........................28

2.3 Differential segment of regenerator with energy terms indicated .............................30

2.4 Numerical grid used for fluid temperature solution................................................32

2.5 Numerical grid used for regenerator temperature solution ........................................33

2.6 Mass flow rate variation for an idealized regenerator .............................................40

2.7 Numerical model predictions and published results for $\varepsilon_{\mathrm{t}}$ as a function of NTU and various values of $\mathrm{U}$ in the ideal regenerator limit ........................................42

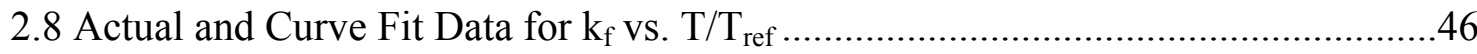

2.9 Specific heat as a function of temperature for some possible working fluids...........47

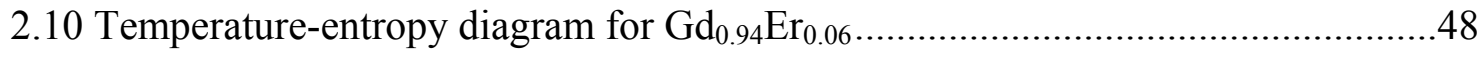


2.11 Partial derivative of entropy with respect to applied field based on data and

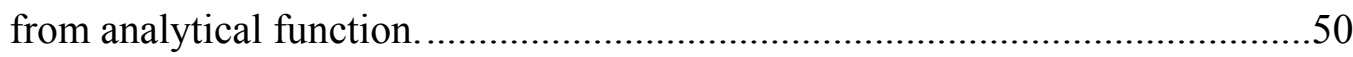

2.12 Comparison of Nusselt number correlations.........................................................

2.13 Comparison of effective static conductivity models under typical AMRR

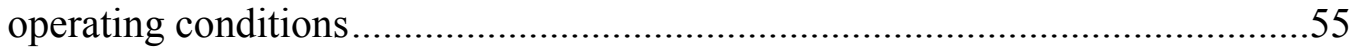

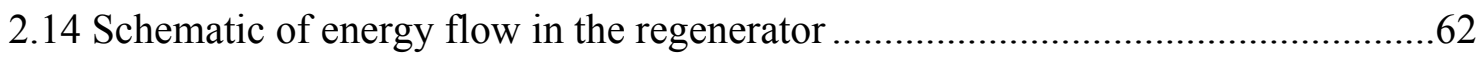

2.15 Differential segment for energy flow................................................................63

2.16 Schematic of entropy flow in the regenerator.....................................................64

2.17 Differential segment for entropy flow ................................................................64

2.18 Enthalpy flux plot for a steady-state cycle........................................................67

2.19 T-s Diagram of a Differential Regenerator Segment..............................................69

3.1 Schematic of the passive regenerator test setup......................................................73

3.2 Predicted results from the lumped capacitance and modified lumped capacitance models as a function of the experimentally measured results for Test Set A

3.3 Predicted results from the lumped capacitance and modified lumped capacitance models as a function of the experimentally measured results for Test Set B.

4.1 Predicted refrigeration per cycle as a function of the number of time steps (m) for various values of the number of axial steps (n)

4.2 Predicted refrigeration per cycle as a function of number of time steps (m) used in the simulation for a non-layered bed and the curve fit used to extrapolate to the true solution

4.3 Refrigeration per cycle as a function of number of time steps $(\mathrm{m})$ for a layered bed. .85

4.4 Refrigeration capacity and COP of a $25 \mathrm{~L}$ layered and non-layered bed with an aspect ratio of 0.34 as a function of the water mass flow rate.

4.5 $\mathrm{COP}$ of a layered bed producing function of the aspect ratio. .89

4.6 Hot heat exchanger schematic 
4.7 Heat exchanger model flow chart ...................................................................94

4.8 Condensing heat exchanger solution flow chart ...................................................99

4.9 Heat transfer rate as a function of the air flow rate predicted by the

DOE/ORNL Mark VI Model and the heat transfer analogy method.................102

4.10 Sensible heat ratio as a function of the air flow rate predicted by the

DOE/ORNL Mark VI Model and the heat transfer analogy method...

$4.11 \mathrm{COP}$ of a layered and non-layered bed producing $8.76 \mathrm{~kW}$ of refrigeration at its optimal aspect ratio. Also shown is the COP for a comparable vapor compression cycle.

4.12 COP of a $10 \mathrm{~L}$ layered and $25 \mathrm{~L}$ non-layered bed producing $8.76 \mathrm{~kW}$ of refrigeration at their optimal aspect ratios as a function of heat rejection temperature 108

4.13 COP of a layered and non-layered bed producing $150 \mathrm{~W}$ of refrigeration at its optimal aspect ratio. Also shown is the COP for a comparable vapor compression cycle

4.14 COP of a $0.7 \mathrm{~L}$ layered and 3.5 $\mathrm{L}$ non-layered bed producing $150 \mathrm{~W}$ of refrigeration at their optimal aspect ratios as a function of load temperature

4.15 COP of a $0.7 \mathrm{~L}$ layered and $3.5 \mathrm{~L}$ non-layered bed operating at their optimal aspect ratios and with constant mass flow rates as a function of load temperature

4.16 Cooling capacity of a $0.7 \mathrm{~L}$ layered and 3.5 L non-layered bed operating at their optimal aspect ratios and with constant mass flow rates as a function of load temperature

5.1 Entropy as a function of temperature for $\mathrm{La}\left(\mathrm{Fe}_{\mathrm{x}} \mathrm{Si}_{1-\mathrm{x}}\right)_{13} \mathrm{H}_{\mathrm{y}}$ from Fujita et al.(2003).....

5.2 Predicted entropy as a function of temperature for advanced material using GdEr properties with a scaling factor

$5.3 \mathrm{COP}$ of a layered advanced regenerator producing $8.76 \mathrm{~kW}$ of refrigeration versus regenerator porosity. Note that an optimal porosity of 0.55 
indicates that the channels and the material between the channels will be

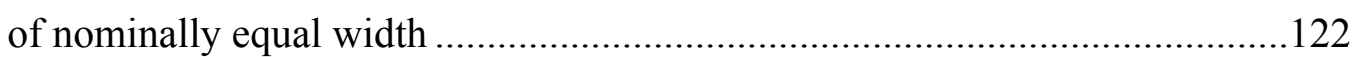

$5.4 \mathrm{COP}$ of an advanced regenerator capable of producing $8.76 \mathrm{~kW}$ of refrigeration at its optimal aspect ratio at conditions given in Table Table 5.1 with a porosity of 0.55 . Also shown is the COP for a comparable vapor compression cycle

5.5 COP of an advanced regenerator and layered and non-layered packed sphere regenerators each capable of producing $8.76 \mathrm{~kW}$ of refrigeration at its optimal aspect ratio 


\section{List of Tables}

2.1 Coefficients of property functions for water.........................................................4

2.2 Coefficients of property functions for ethylene glycol and water solution ...............44

2.3 Coefficients of property functions for propylene glycol and water solution.............45

3.1 Model inputs used to match the passive regenerator test results ..............................74

3.2 Experimentally measured and predicted passive regenerator results under conditions associated with Test Set A. ...........................................................76

3.3 Experimentally measured and predicted passive regenerator results for Test

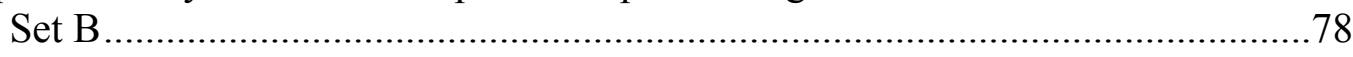

3.4 Passive regenerator test instrumentation and model inputs and associated

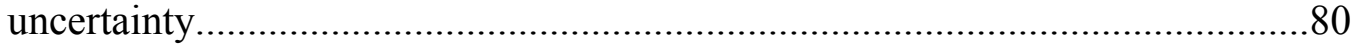

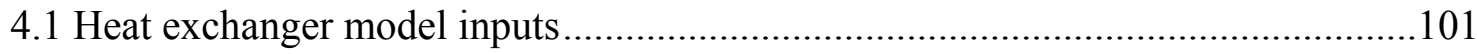

4.2 Parameters used for space cooling comparative study ............................................104

4.3 Parameters used for refrigeration analysis .........................................................110

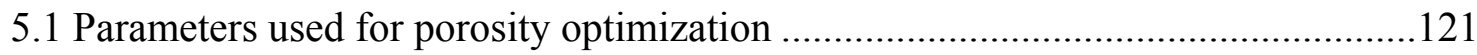

5.2 Parameters used for space cooling parametric study .............................................126

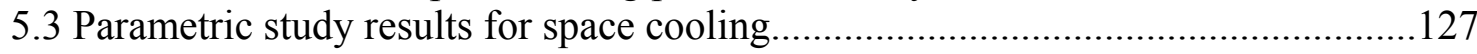

5.4 Model sensitivity to correlations and material properties ........................................128 


\title{
List of Variables
}

\author{
$\mathrm{a}_{\mathrm{s}} \quad$ surface area of regenerator bed \\ $\mathrm{A}_{\mathrm{c}} \quad$ cross sectional area of regenerator bed $\left(\mathrm{m}^{2}\right)$ \\ B wall thickness between flow passages $(\mathrm{m})$ \\ Bi Biot number \\ C Curie constant, heat capacity rate $(\mathrm{W} / \mathrm{K})$ \\ $\mathrm{C} 1 \quad$ constant used to evaluate Curie-Weiss equation of state \\ $\mathrm{C}_{1} \quad$ constant of integration \\ $\mathrm{C}_{2} \quad$ constant of integration \\ COP coefficient of performance \\ $\mathrm{C}_{\mathrm{r}} \quad$ ratio of heat capacity rates \\ $\mathrm{c}_{\mathrm{s}} \quad$ specific heat of air for condensing heat exchanger analysis $(\mathrm{J} / \mathrm{kg}-\mathrm{K})$ \\ $\mathrm{c}_{\mu \mathrm{oH}} \quad$ specific heat of the matrix material at constant magnetic field $(\mathrm{J} / \mathrm{kg}-\mathrm{K})$ \\ $c_{\mu o H=0}$ specific heat of the matrix material at 0 T magnetic field $(\mathrm{J} / \mathrm{kg}-\mathrm{K})$ \\ $\mathrm{D}^{\mathrm{d}} \quad$ dispersion coefficient \\ $\mathrm{d}_{\mathrm{h}} \quad$ hydraulic diameter $(m)$ \\ f friction factor \\ $\dot{g}^{\prime \prime \prime} \quad$ internal heat generation $\left(\mathrm{W} / \mathrm{m}^{3}\right)$ \\ $\mathrm{h} \quad$ specific enthalpy $(\mathrm{J} / \mathrm{kg})$, convection heat transfer coefficient $\left(\mathrm{W} / \mathrm{m}^{2}-\mathrm{K}\right)$ \\ $\mathrm{h}_{\mathrm{eff}} \quad$ effective heat transfer coefficient of the regenerator $\left(\mathrm{W} / \mathrm{m}^{2}-K\right)$ \\ $\mathrm{k} \quad$ thermal conductivity $(\mathrm{W} / \mathrm{m}-\mathrm{K})$ \\ $\mathrm{k}_{\mathrm{ef}} \mathrm{f} \quad$ effective thermal conductivity of regenerator and fluid (W/m-K) \\ $\mathrm{k}_{\text {static }}$ effective conductivity of regenerator and fluid with zero mass flow rate \\ $(W / m-K)$ \\ $\mathrm{H} \quad$ applied field $(\mathrm{A} / \mathrm{m})$ \\ i spatial subscript \\ $\mathrm{j} \quad$ temporal subscript \\ $\mathrm{J} \quad$ function of temperature only used to develop a magnetic equation of state \\ $\mathrm{K}$ function of applied field only used to develop a magnetic equation of state \\ $\mathrm{L} \quad$ length $(\mathrm{m})$ \\ $\mathrm{m} \quad$ number of time steps used in numerical solution \\ $\dot{m} \quad$ mass flow rate $(\mathrm{kg} / \mathrm{s})$ \\ M magnetic intensity $(\mathrm{A} / \mathrm{m})$ \\ $\mathrm{n} \quad$ number of axial control volumes used in numerical solution \\ $\mathrm{n}_{\text {bed }} \quad$ number of beds in the rotary regenerator \\ NTU number of transfer units \\ $\mathrm{Nu} \quad$ Nusselt number \\ $\mathrm{P} \quad$ pressure $(\mathrm{Pa})$ \\ $\mathrm{Pe} \quad$ Peclet number \\ Pr Prandtl number \\ Q heat transfer $(J)$ \\ $\dot{q} \quad$ heat transfer rate $(W)$ \\ $\mathrm{r} \quad$ radius $(m)$ \\ $\mathrm{R} \quad$ Resistance, ratio of fluid heat capacity to the regenerator heat capacity
}




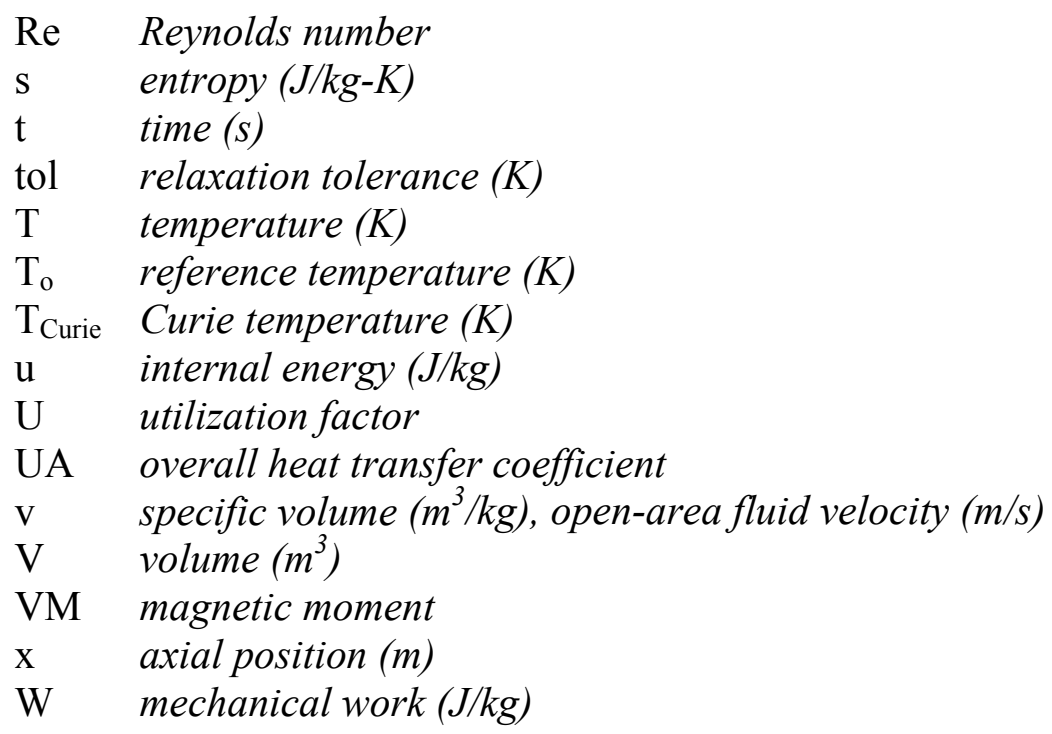

\section{Greek}

$\alpha \quad$ parameter from Ergun equation

$\beta \quad$ constant related to packing of spheres in regenerator bed

$\Delta \quad$ change

$\delta \quad$ small change, used for numerical integration

$\varepsilon \quad$ porosity of matrix, heat exchanger effectiveness

$\varepsilon_{\mathrm{t}} \quad$ thermal effectiveness

$\phi \quad$ constant used to calculate $k_{\text {eff }}$

$\eta \quad$ efficiency

$\mu \quad \operatorname{viscosity}\left(N-s / m^{2}\right)$

$\mu_{0} \mathrm{H} \quad$ applied field (Tesla)

$\mu_{0} \mathrm{H}_{0} \quad$ reference applied field (Tesla)

$\rho \quad \operatorname{density}\left(\mathrm{kg} / \mathrm{m}^{3}\right)$

$\sigma \quad$ entropy production $(\mathrm{J} / \mathrm{kg}-\mathrm{K})$

$\tau \quad$ cycle duration $(s)$

\section{$\underline{\text { Subscripts }}$}
back back
cond conduction
conv convection
$\mathrm{C} \quad$ cold or refrigeration temperature
eff effective
exp experimentally determined value
f fluid
flux through-flow
front front
$\mathrm{H} \quad$ hot or heat rejection temperature
$\mathrm{HX}$ heat exhanger 


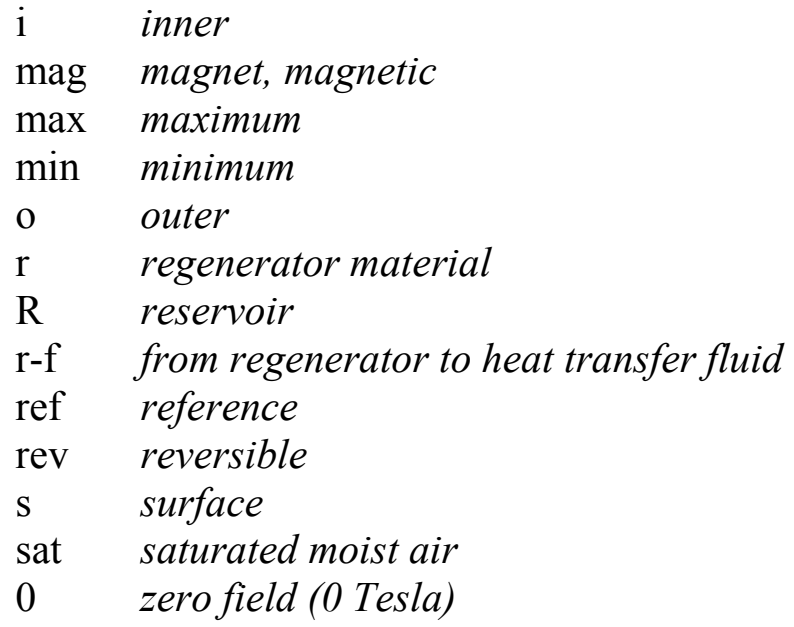

\section{$\underline{\text { Superscripts }}$}

* guess value, modified value

$+\quad$ new value of guess value

, per unit length, modified value 



\section{Chapter 1 Introduction}

\subsection{The Magnetocaloric Effect}

The thermal and magnetic properties of some substances are highly coupled over certain, typically limited operating ranges, allowing them to be used within energy conversion systems. A thermodynamic substance can change its internal energy $(U)$ as a result of either a work transfer or a heat transfer, leading to the differential energy balance:

$$
d U=T \cdot d S+d W
$$

The $1^{\text {st }}$ term in Eq. (1) corresponds to an inflow of heat $(T d S)$ and the $2^{\text {nd }}$ to an inflow of work $(d W)$. In general, work can flow in many forms (e.g., mechanical, electrical, etc.). The familiar fundamental property relationship results when only volumetric compression work $(P-V)$ is considered:

$$
d U=T \cdot d S-P \cdot d V
$$

Temperature $(T)$ and entropy $(S)$ form what in thermodynamics is called a canonical conjugate property pair that, together, defines the transfer of heat. Pressure $(P)$ and volume $(V)$ form another such property pair defining the transfer of mechanical work. If hysteresis is ignored, then it is possible to define a similar pair of properties to describe the transfer of magnetic work: applied field $\left(\mu_{o} H\right)$ and magnetic moment $(V M)$, Guggenheim (1967).

$$
d U=T \cdot d S+\mu_{o} \cdot H \cdot d(V \cdot M)
$$

Examination of Eq. (1.3) reveals that the applied field is analogous to pressure and magnetic moment is analogous to (the inverse of) volume. This analogy is physically 
revealing; increasing the applied field tends to align the magnetic dipoles, which can be thought of as small torsional springs, and the result is an increase in the magnetization. The process of aligning the magnetic dipoles requires work and reduces entropy. In a compressible substance, increasing the pressure reduces the space between molecules, which is analogous to compressing small linear springs. Reducing the volume requires work and reduces entropy. This analogy is illustrated in Figure 1.1.
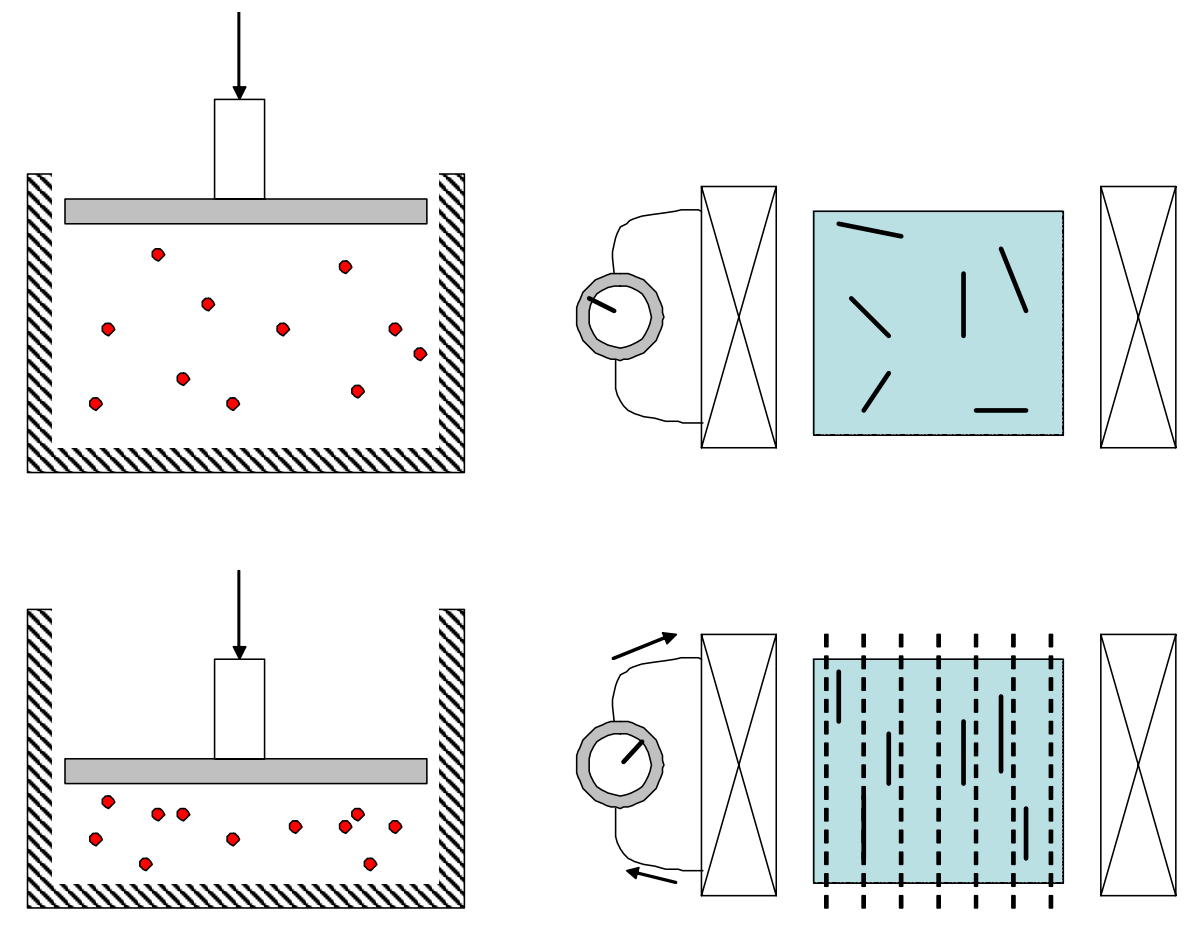

mechanical work transfer to a compressible subtance magentic work transfer to a magnetic subtance

Figure 1.1. Analogy between magnetic and mechanical work transfer

Using the analogy described above, it becomes possible to apply all of the typical thermodynamic results and identities ordinarily used with a pure compressible substance to a magnetic material; including Maxwell's relations that describe relationships between 
partial derivatives of properties and the idea of an equation of state that describes the magnetization as a function of temperature and applied field. A temperature-entropy diagram for a magnetic material will therefore include lines of constant applied magnetic field rather than pressure but is otherwise analogous to a similar diagram for air or any other compressible working fluid. Figure 1.2 illustrates the temperature-entropy diagram for $\mathrm{Gd}_{0.94} \mathrm{Er}_{0.06}$.

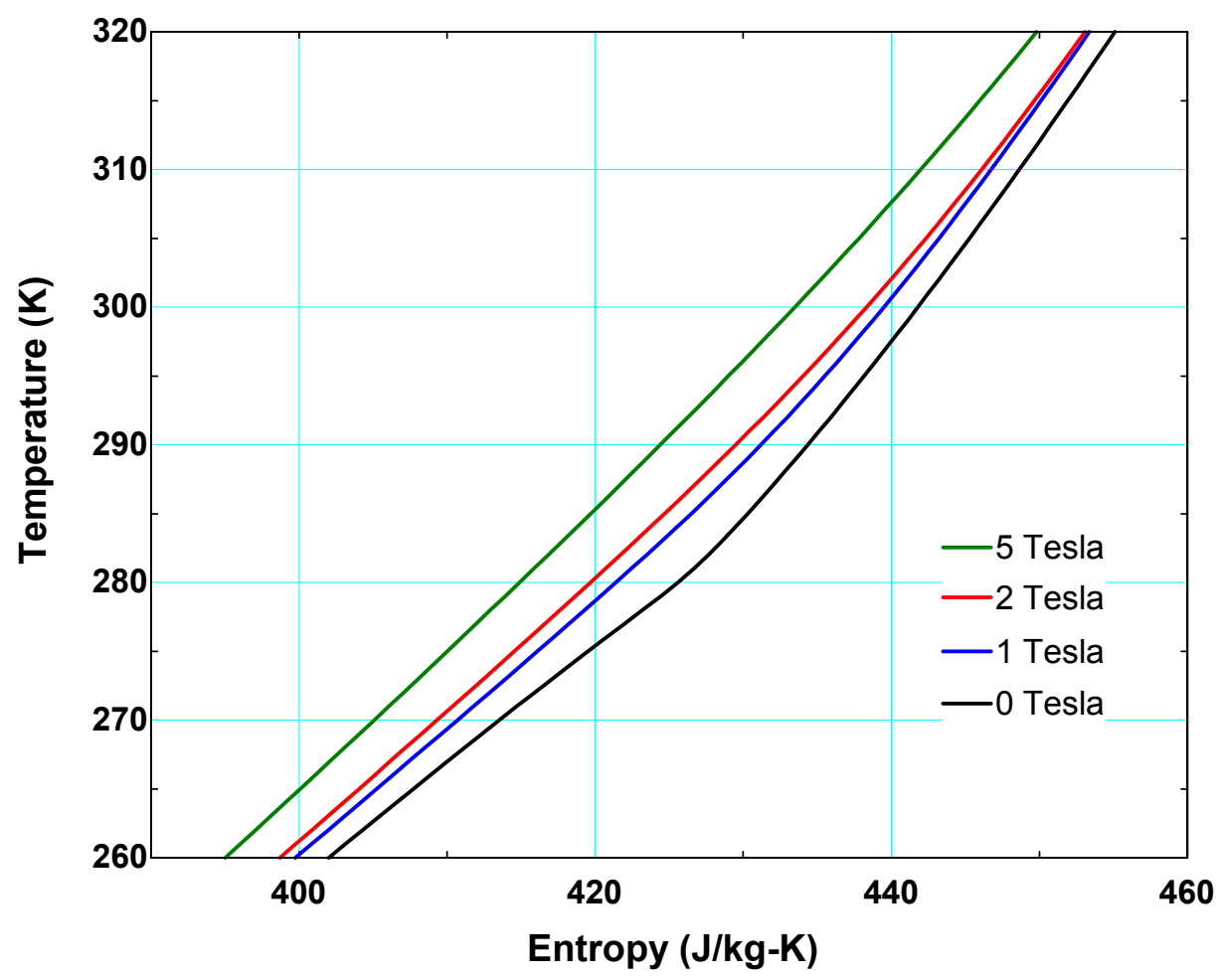

Figure 1.2. Temperature-entropy diagram for $\mathrm{Gd}_{0.94} \mathrm{Er}_{0.06}$

Closer examination of Figure 1.2 reveals that it is possible to change the temperature of a magnetic material in an adiabatic process by changing the applied magnetic field. Figure 
1.3 illustrates the adiabatic temperature change of an alloy of $94 \%$ Gadolinium and $6 \%$ Erbium, $\mathrm{Gd}_{0.94} \mathrm{Er}_{0.06}$, when the magnetic field is increased from 0 Tesla to 2 or 5 Tesla. Figure 1.3 shows that the adiabatic temperature change (magnetocaloric effect) depends on the initial temperature of the material, and that a high magnetocaloric effect occurs only over a relatively limited temperature span. The temperature at which the maximum magnetocaloric effect occurs is referred to as the Curie point of the material. Neglecting magnetic hysteresis, adiabatic magnetization and demagnetization are isentropic processes in the thermodynamically reversible limit. Therefore, when the material is demagnetized, its temperature will decrease to its zero-field state.

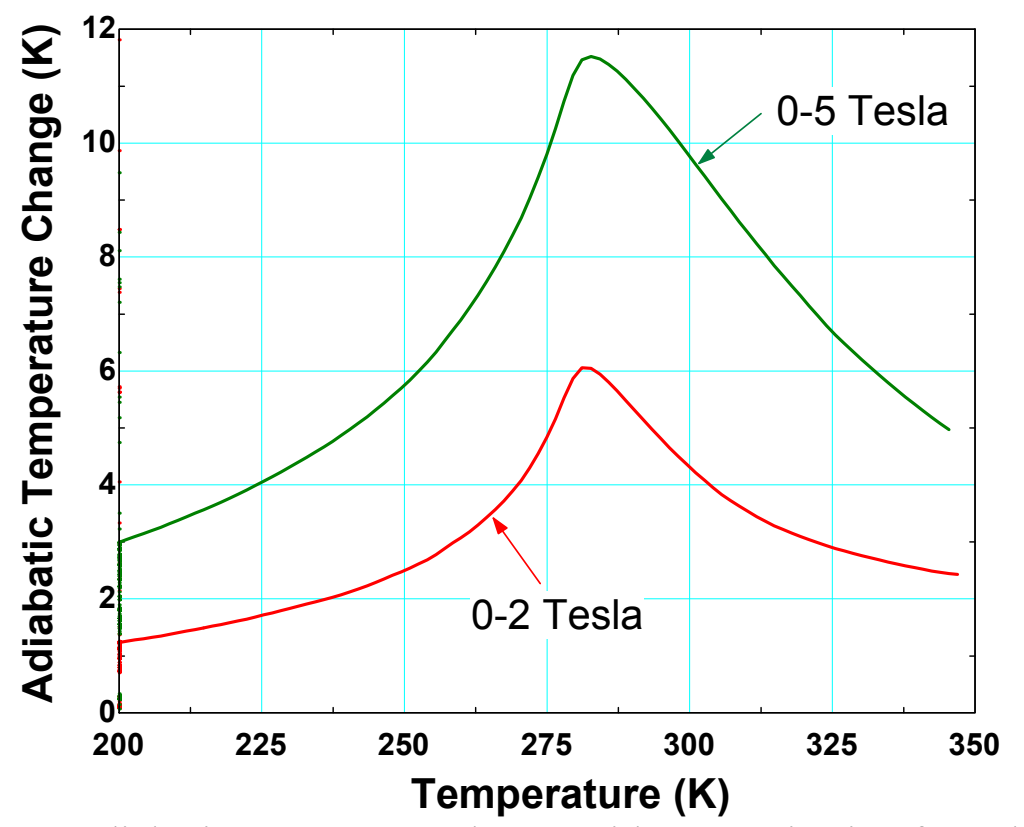

Figure 1.3. Adiabatic temperature change with magnetization for $\mathrm{Gd}_{0.94} \mathrm{Er}_{0.06}$

\subsection{Magnetic Refrigeration}

Materials that exhibit the magnetocaloric effect described in section 1.1 are thermodynamically coupled; their entropy depends not only on temperature but also on 
some other quantity, in this case magnetic field. Therefore, these materials can be forced to undergo various types of thermodynamic cycles that cause entropy to move from a low temperature to a high temperature due to a work input. Magnetic cooling is an alternative refrigeration technology that may be competitive with vapor compression systems. The metallic refrigerant has essentially zero vapor pressure and therefore is ecologically sound with no Ozone Depletion Potential (ODP) and zero direct Global Warming Potential (GWP). Vapor compression is a mature technology with well-known fundamental performance limits resulting from the throttling process, the compression process, and imbalances in the condenser and evaporator. Throttling and compression losses are avoided in a magnetic system, although new dissipation mechanisms are encountered in their place. In theory, a well-designed magnetic system may be competitive with or even more efficient than vapor compression systems. The objective of this thesis is to investigate the practical limits of the efficiency of a magnetic cooling cycle; this section describes various thermodynamic cycle alternatives that may be implemented using a magnetocaloric substance.

Giauque and MacDougall (1933) used a magnetic cooling system to reach temperatures below $1 \mathrm{~K}$, breaking the temperature barrier that was previously imposed by the properties of compressible fluids. They and other researchers used a "one-shot" refrigeration technique which consisted of a solid piece of magnetocaloric alloy and utilized an isothermal magnetization, in which the material is put in contact with a hot 
reservoir, followed by an adiabatic demagnetization. Figure 1.4 shows a one-shot refrigeration cycle on a T-s diagram.

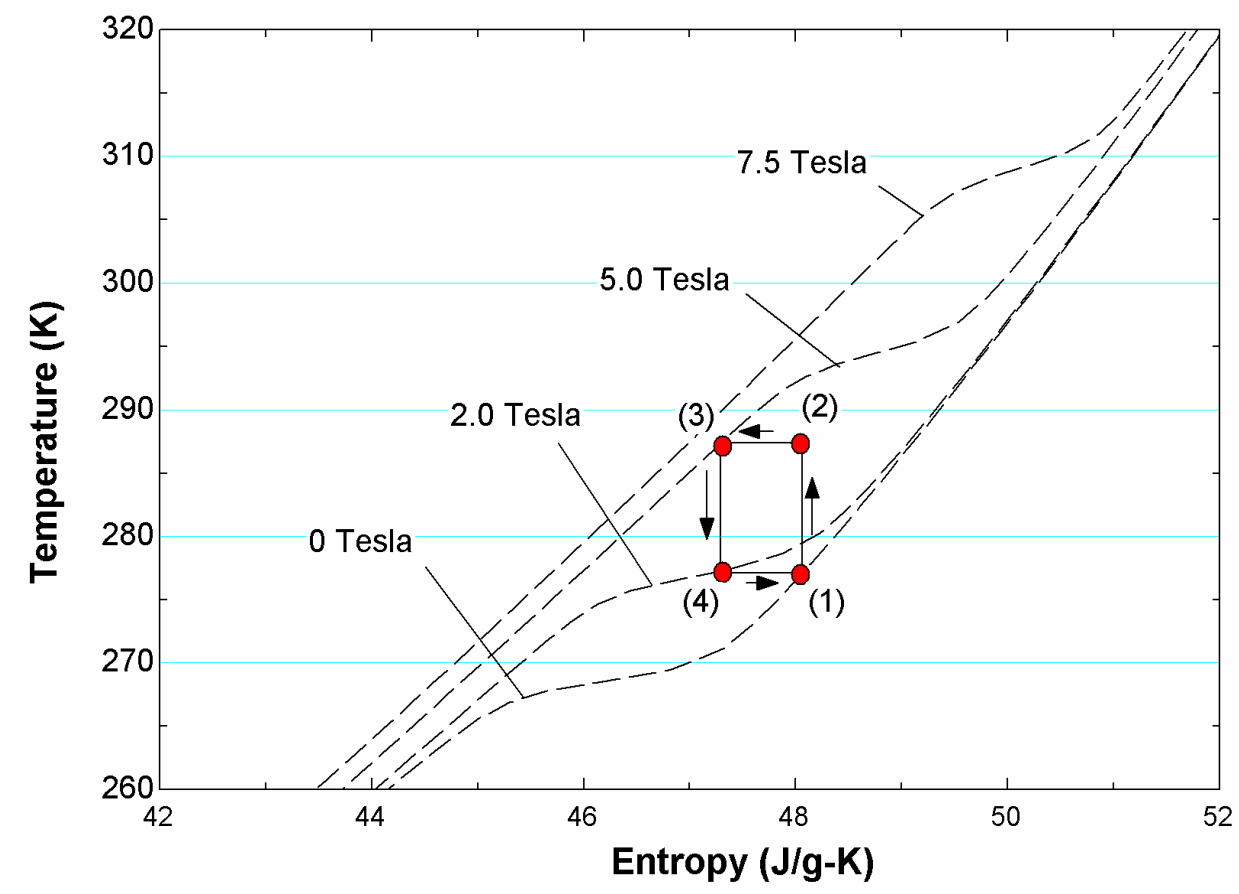

Figure 1.4. Temperature-entropy diagram for a one-shot refrigeration cycle

In Figure 1.4, process (1) to (2) is an adiabatic magnetization, which is followed by isothermal magnetization, (2) to (3), where heat is rejected to the hot reservoir. (3) to (4) is an adiabatic partial demagnetization which lowers the temperature of the material to that of the cold reservoir. A cooling load is accepted by the magnetocaloric material from the cold reservoir during the isothermal demagnetization process from (4) to (1).

More advanced, continuously operating versions of adiabatic demagnetization refrigeration systems have been developed. A rotating wheel configuration has been 
demonstrated at Los Alamos National Lab according to Steyart (1978), the Hughes Aircraft Company, and by researchers in Grenoble, France. In these systems, a mass of magnetic material is rotated sequentially through a cold thermal reservoir, an adiabatic magnetic field, a warm thermal reservoir, and an adiabatic region with no magnetic field. The process undergone by the magnetic material approaches the Carnot cycle that is shown in Figure 1.4. Reciprocating devices have also been fabricated at Los Alamos National Lab - Barclay et al. (1979), the Jet Propulsion Lab - Johnson (1984), the David Taylor Naval Research Center Patton et al. (1986), and by researchers in Grenoble France - Lacaze et al. (1986). In these systems, the material is linearly moved into and out of a magnetic field and into and out of contact with two thermal reservoirs. Again, the theoretically optimal cycle approaches the Carnot limit. More recently, researchers at NASA - King et al. (2002) - and elsewhere - Numazawa et al. (1986) - have used ramped field configurations with static heat switches to accomplish the same cycle.

These cycles all suffer from limited temperature lift related to the fact that all of the magnetocaloric material undergoes the same thermodynamic cycle. Figure 1.3 shows that for $\mathrm{Gd}_{0.94} \mathrm{Er}_{0.06}$, the maximum temperature change that can be induced in this material with a 5 Tesla field change is approximately $11 \mathrm{~K}$; this corresponds to the absolute maximum temperature difference that can be sustained between the two reservoirs and is much too low to be useful for most refrigeration applications. These cycles also require complex heat switches with limited capacities that are not practical for near room temperature, commercial devices. 


\subsection{Active Magnetic Regenerative Refrigeration (AMRR) Cycle}

The technical barriers described in section 1.2 have been overcome by the active magnetic regenerative refrigeration (AMRR) cycle. A porous, packed bed of magnetic material is exposed to a time-varying magnetic field and a time-varying flow of heat transfer fluid. Different mechanical realizations of this cycle are possible. For example, the magnetic material may be stationary and the field varied by controlling the current in a solenoid. This configuration is feasible at very low temperatures where superconductors can be used to efficiently handle the large currents that are required and a number of implementations of this idea have been analyzed and tested. At cryogenic temperatures, researchers at MIT - Taussig et al. (1986) - have built and tested several systems, including one in which the magnetic regeneration cycle is implemented in addition to the typical Gifford-McMahon cycle in order to simultaneously realize both magnetic and mechanical cooling according to Nellis and Smith (1998). Other researchers in France from Seyfert et al. (1998) and Japan form Matsumoto and Hashimoto (1989) have built similar systems at cryogenic temperatures.

The AMRR concept has allowed magnetic cooling to be extended to near room temperature by removing the limitation on the overall temperature swing. Typically, the applied field variation is achieved by physically moving the magnetic regenerator into and out of a stationary magnetic field either linearly as described by $\mathrm{Wu}$ (2003) or rotationally as shown by Zimm et al. (2002). An AMRR constructed at Los Alamos 
National Lab in collaboration with Astronautics, Blumenfeld et al. (2002), utilizes a high temperature superconducting solenoid to generate a time-varying magnetic field over a stationary bed of pure gadolinium spheres and was able to achieve a COP that was competitive with vapor compression systems (note that the COP reported for this system was defined as the cooling provided over the power delivered to the magnetic material and therefore neglected the power required to cool the HTS magnet, inefficiencies within the magnet power supply, and other parasitic power consumptions).

A very simplified version of the AMRR cycle is illustrated in Figure 1.5. The porous regenerator bed consists of a magnetic material and initially has a quasi-linear temperature profile from the warm reservoir temperature to the cold reservoir temperature, shown in state (1). In the magnetization process, (1) to (2), the magnetic field in the bed is increased with no fluid flow which causes the temperature of the material everywhere in the bed to increase due to the magnetocaloric effect, as shown in state (2). The temperature of the material at the hot end of the bed rises above the hot reservoir temperature. In the cold-to-hot flow process, the heat transfer fluid is pushed through the bed from the cold to the hot end, causing the bed temperature to decrease as it contacts the cooler fluid as shown in state (3). During this process, fluid at a temperature above the hot reservoir temperature is pushed from the hot end of the bed, rejecting heat. At the end of this process the material temperature may have returned to its original distribution. Now the bed is demagnetized so that the temperature everywhere decreases as shown in state (4). Finally, the heat transfer fluid is pushed back through the bed from 
the hot to the cold end. This hotter fluid cools the bed back to its original temperature distribution. The fluid is pushed out of the cold end at a temperature below the cold reservoir temperature, producing a cooling load. 


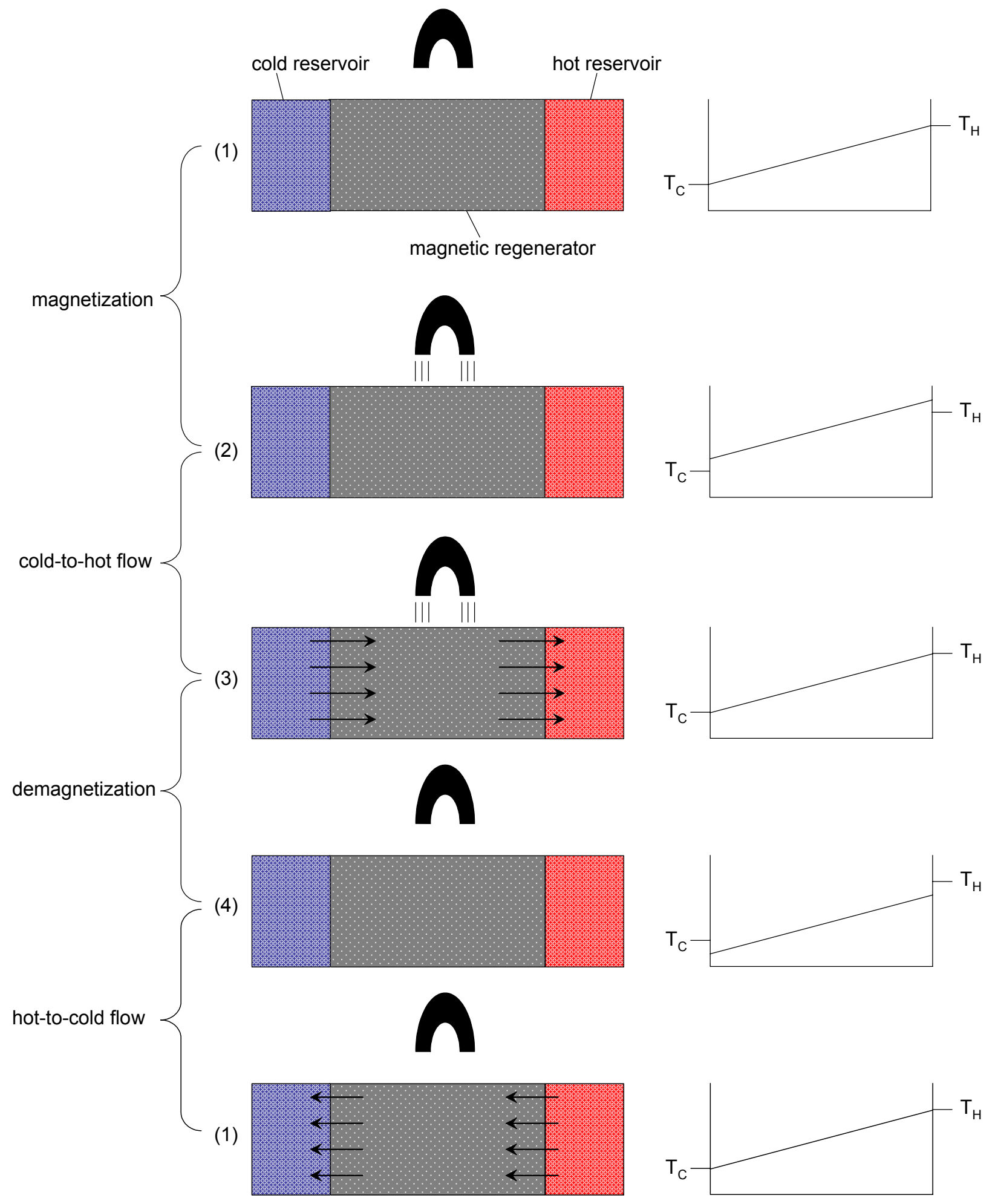

Figure 1.5. Active magnetic regenerative refrigeration cycle 
At Astronautics, an AMRR system has been constructed in which a wheel with multiple regenerators composed of packed gadolinium spheres is rotated through the gap of a 1.5 Tesla permanent magnet. Figure 1.6 is a photograph of the device. The wheel is divided into six, separate active magnetic regenerator (AMR) beds. The beds are subjected to an oscillating flow of heat transfer fluid (water) that is correctly phased relative to the position of the bed in the magnetic field. Fluid flow is provided by a single pump, and fluid exiting the hot side of a bed is sent through a heat rejection heat exchanger before it enters the hot side of a regenerator on the opposite side of the wheel. Therefore, fluid exiting a bed during a cold-to-hot flow exits the bed at an elevated temperature, is cooled to the hot reservoir (in this case ambient air) temperature, and is sent into a bed that is experiencing hot-to-cold flow. The flow setup is analogous for fluid exiting and entering the cold side of the regenerator beds. This flow configuration is enabled by a sophisticated valve system; each bed contains its own fluid manifold system that passes under a distribution and recovery point at a specific angular location. 


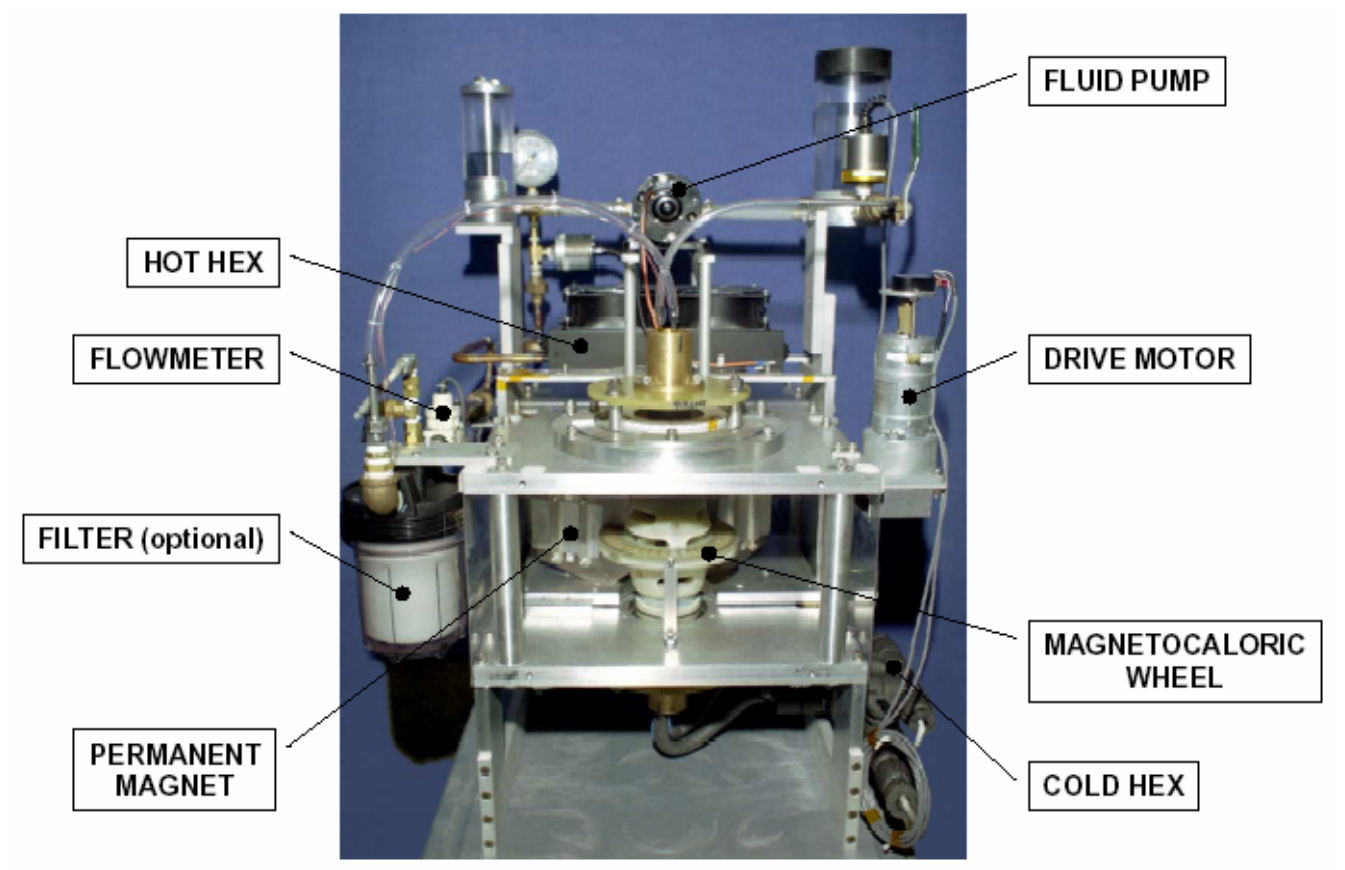

Figure 1.6. Rotary AMRR at Astronautics

The rotary AMRR system undergoes the same cycle shown in Figure 1.5. The rotary regenerator considered is a ring that contains several regenerator beds which are aligned back-to-back (i.e. the cold and hot ends of adjacent beds are adjacent). Flow through the bed is in the tangential direction. The regenerator beds spin at a constant angular velocity $(\omega)$ through a permanent magnetic that covers a specified arc over the regenerator bed. Due to the back-to-back nature of the bed configuration, there are two types of beds in the device and these beds experience slightly different variations in applied field over a cycle. In an A-type bed, the hot end of the regenerator enters the magnetic field and therefore the bed is magnetized from the hot end to the cold end. Figure 1.7 demonstrates how an A-type bed enters the magnetic field. The Type A bed is highlighted in the figure, and the disk is rotating in the clockwise direction so that the bed is drawn into the magnetic field of a fixed $120^{\circ}$ permanent magnet. Figure 1.7 demonstrates process (1) - 
(2) in Figure 1.5, showing approximately half the bed in the magnetic field and half outside the magnetic field. The cold end of Type B bed enters the magnetic field and therefore the bed is magnetized from the cold end to the hot end. The hot and cold side of each bed is labeled with a $C$ or $H$.

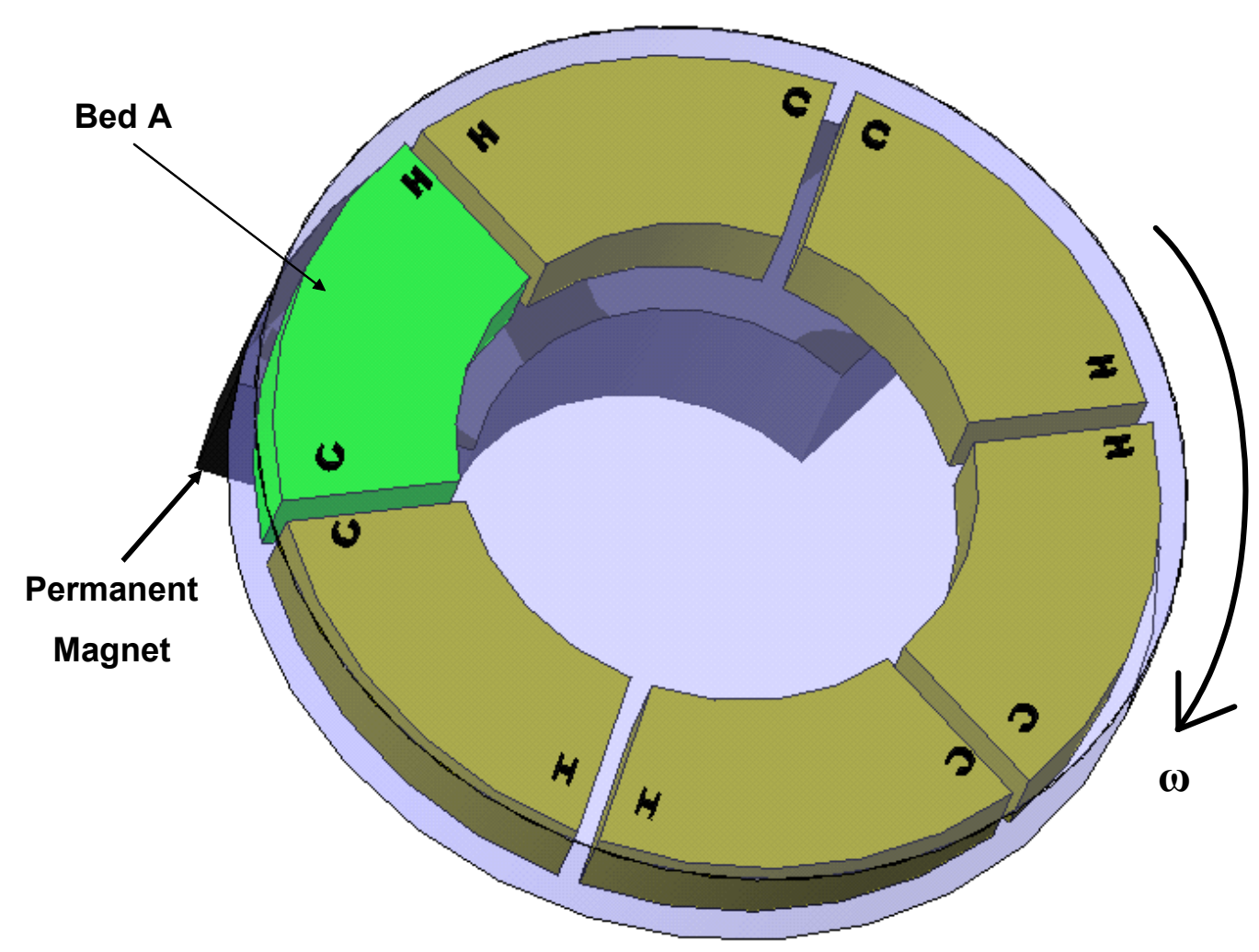

Figure 1.7. Type A bed entering the magnetic field and being magnetized from hot-tocold

Flow from cold end to the hot end of either type of bed is assumed to occur while the entire bed is inside the magnetic field. Note that this cycle configuration is sometimes 
referred to as a Carnot-type cycle because the magnetic field variations are decoupled from the flow processes. Figure 1.8 illustrates a Type A bed fully in the magnetic field and experiencing a flow process, process (2) - (3) in Figure 1.5. The figure also shows a Type B bed entering the magnetic field and being magnetized from its cold end to its hot end.

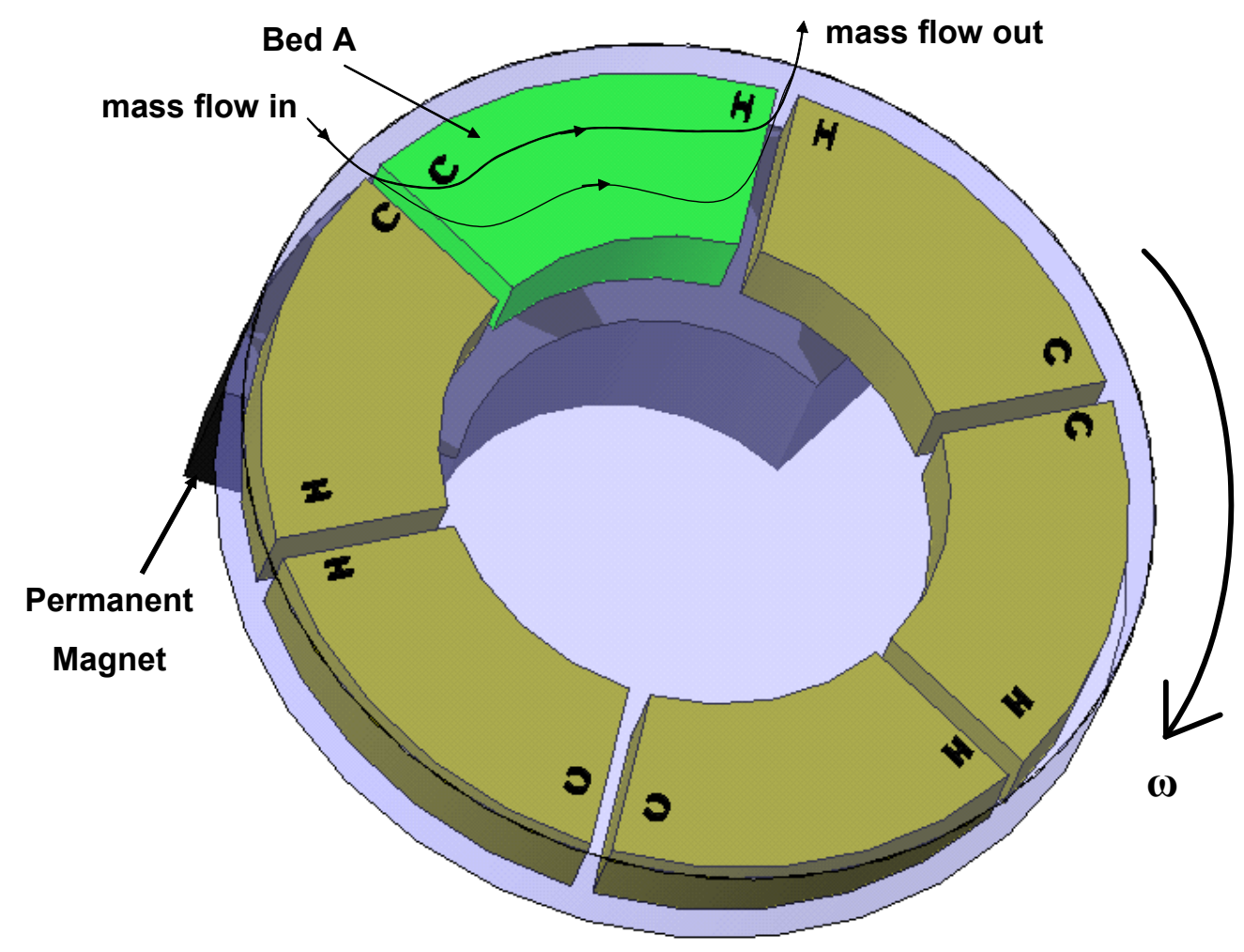

Figure 1.8. Type-A bed fully in the magnetic field and subjected to flow from the cold end to the hot end. Note the Type-B bed entering the magnetic field and being magnetized from its cold end to its hot end. 
Demagnetization is shown in Figure 1.9. The highlighted, Type A bed is leaving the magnetic field (the bed is approximately half in the magnetic field and half outside the magnetic field) and is being demagnetized from its hot end to its cold end, (3) - (4) in Figure 1.5. The demagnetization process causes the bed's temperature to decrease and there is no fluid flow during this process.

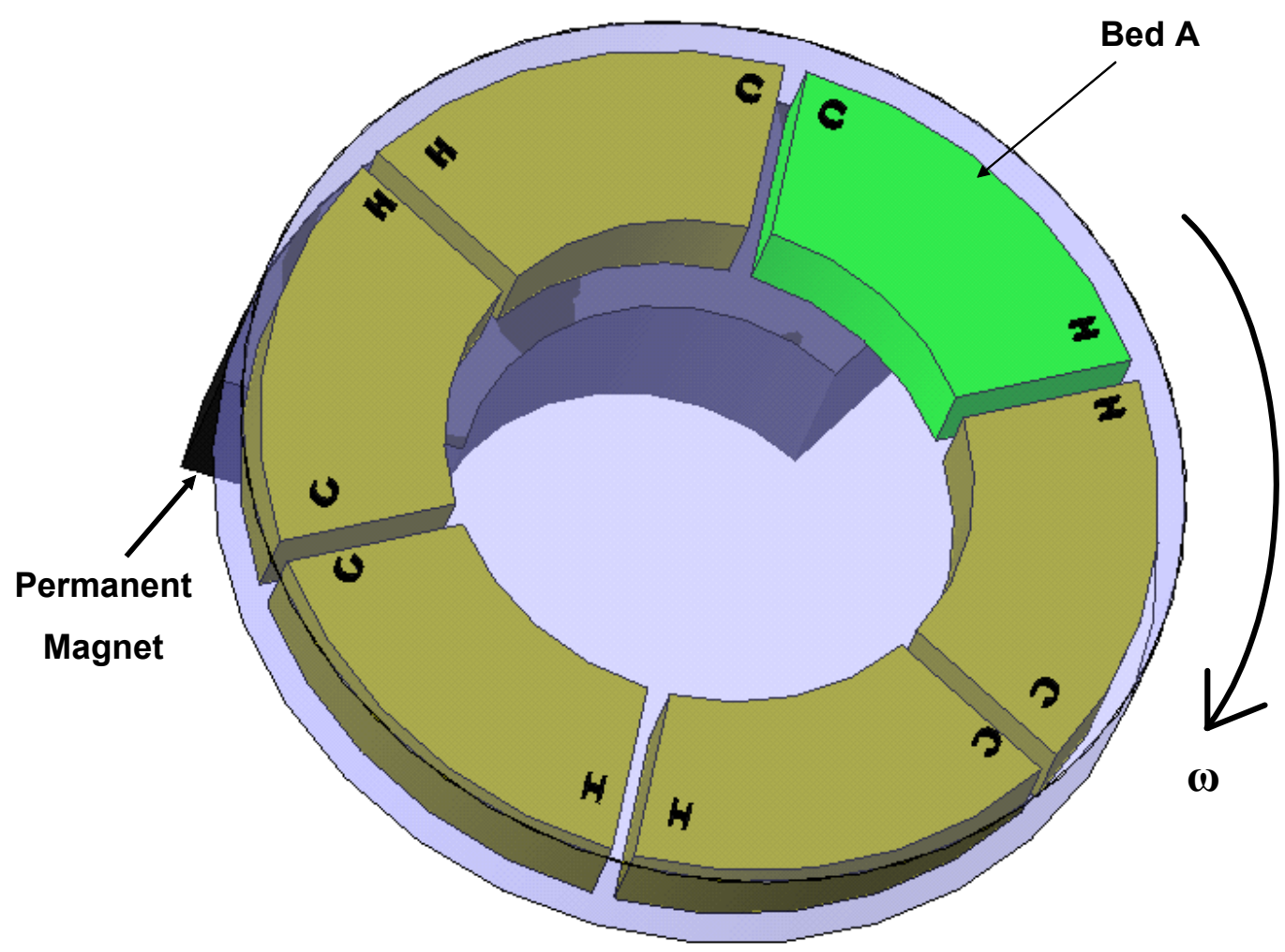

Figure 1.9: Type A bed leaving the magnetic field.

Flow from the hot end to the cold end is assumed to occur while the entire bed is outside the magnetic field; again, consistent with a Carnot type cycle. Figure 1.10 shows the 
Type A outside the magnetic field and subjected to the hot-to-cold flow, (4) - (1) on

Figure 1.5. Hot-to-cold flow occurs under the same conditions for the Type B bed but demagnetization occurs from its cold end to its hot end.

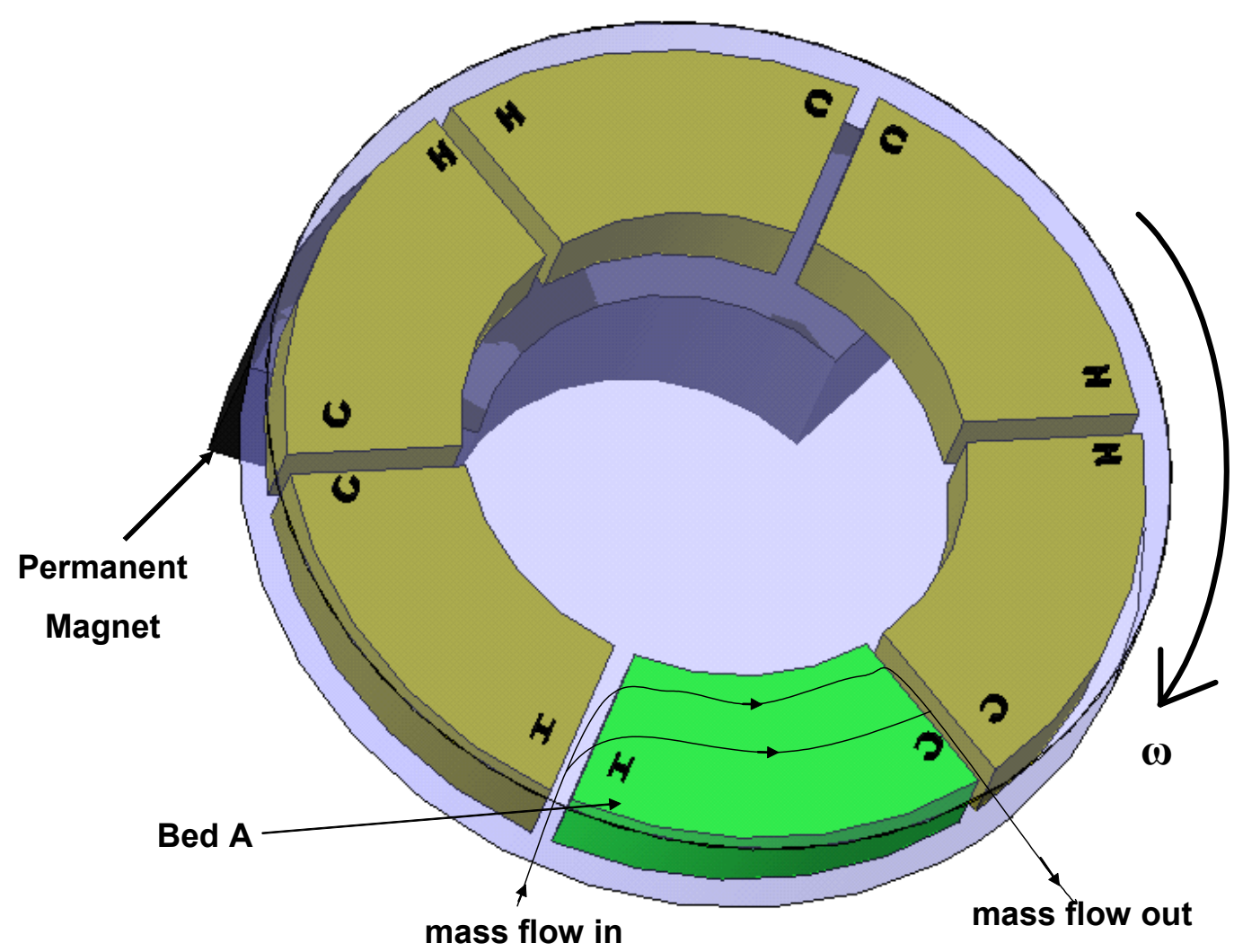

Figure 1.10. Type A Bed experiencing hot-to-cold flow

This system demonstrates a practical implementation of the AMRR cycle described by Zimm et al. (2002). The measured COP of the system is low, primarily due to losses that are concentrated in the regenerator matrix which is composed of small spheres. These spheres provide a large specific area for heat transfer but lead to high pressure loss due to their random and isotropic geometry. The pressure loss limitation leads to low operating 
frequencies and therefore also low cooling power and/or large pumping losses. A bed of spheres is also relatively conductive in the axial direction compared to alternative geometries, which leads to additional parasitic losses. Regenerator matrices composed of parallel plates or, more commonly, screens have been widely adopted over packed beds in cryogenic refrigeration systems. The practical limit to the efficiency that can be realized by an AMRR is limited primarily by losses that can be directly related to the regenerator. These losses can only be accurately investigated through the development of a detailed and realistic model of this component, as described in subsequent chapters.

\subsection{Tunable Magnetocaloric Materials}

Most AMRR matrices that have been fabricated and tested for near room-temperature cooling applications use regenerator beds that are fabricated from pure gadolinium spheres. These matrices exhibit a large magnetocaloric effect (i.e., adiabatic temperature change) over a small temperature range that is centered at the Curie temperature, as shown in Figure 1.11 for a given applied field variation from Ames Laboratory obtained with the permission of Professor Gschneidner . 


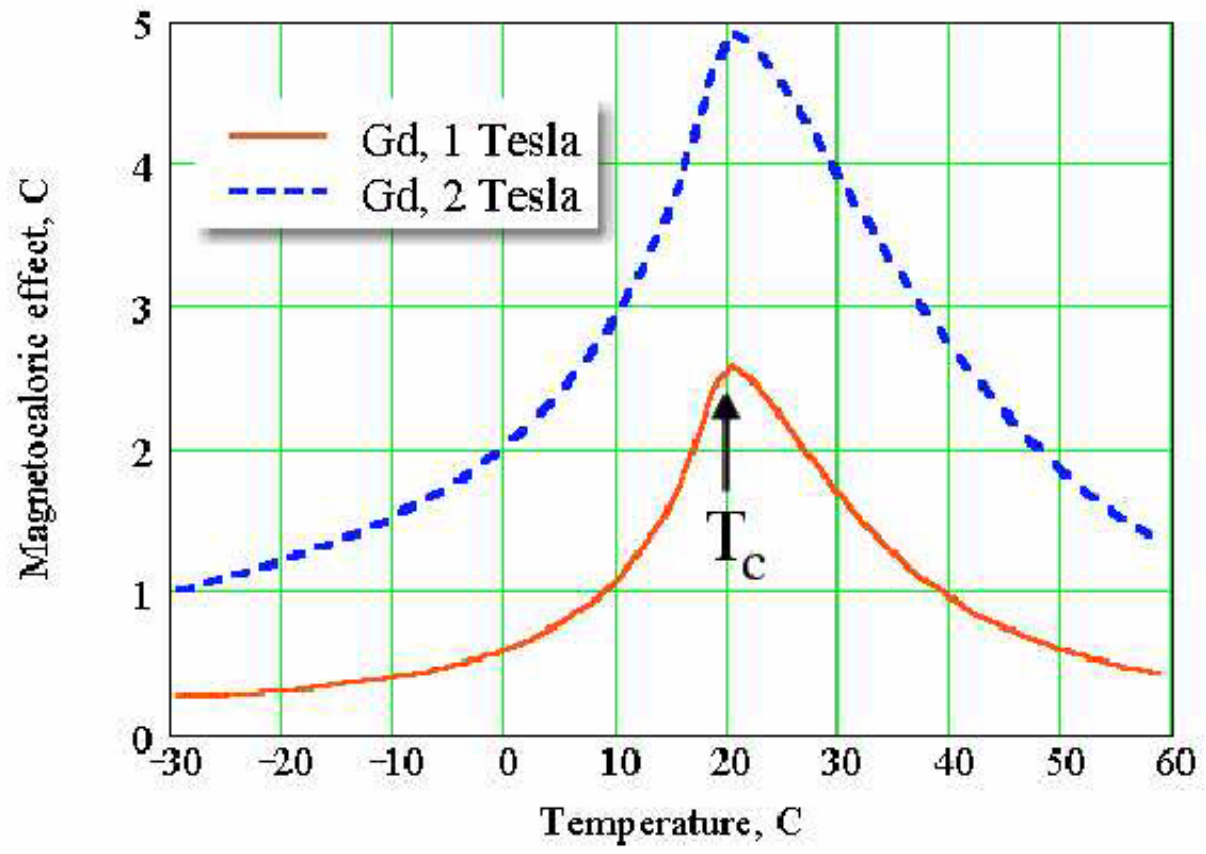

Figure 1.11. Magnetocaloric effect for pure gadolinium (source: Ames Laboratory)

A regenerator bed of pure gadolinium will therefore have a fairly narrow temperature range over which it is useful. The Curie temperature $\left(T_{C}\right)$, and therefore the temperature at which the peak shown in Figure 1.11 occurs, can be adjusted by manipulating the composition of the magnetocaloric material. For example, the Curie temperature can be shifted to lower temperature by alloying with dysprosium and/or erbium without any reduction in the magnetocaloric effect. Also, the operating temperature can be increased to $335 \mathrm{~K}$ by using Si-rich alloy such as $\mathrm{Gd}_{5}\left(\mathrm{Si}_{\mathrm{x}} \mathrm{Ge}_{1-\mathrm{x}}\right)_{4}$ for $1 \leq \mathrm{x} \leq 0.5\left(T_{C}=335 \mathrm{~K}\right.$ for $\mathrm{x}=$ 1, i.e. $\left.\mathrm{Gd}_{5} \mathrm{Si}_{4}\right)$. Examples of alternative materials include the solid solution $\mathrm{Gd}_{5}\left(\mathrm{Si}_{\mathrm{x}} \mathrm{Ge}_{1}\right.$ x) $)_{4}$ for $0 \leq \mathrm{x}<0.5$ developed at Ames Laboratory from Pecharsky and Gschneidner (1997). Other materials that show a similar large and tunable magnetic entropy change include 
$\mathrm{LaFe}_{11.5} \mathrm{Si}_{1.5} \mathrm{H}_{\mathrm{y}}$ from Chen et al. (2003), $\mathrm{MnFeP}_{1-\mathrm{x}} \mathrm{As}_{\mathrm{x}}$ from Tegus et al (2002), $\mathrm{LaFe}_{13-}$ ${ }_{x} \mathrm{Si}_{\mathrm{x}}$ from Hu et al. (2001), and $\mathrm{MnAs}_{1-\mathrm{x}} \mathrm{Sb}_{\mathrm{x}}$ from Wada and Tanabe (1999). Figure 1.12 illustrates the magnetic entropy change for the $\mathrm{LaFe}_{11.5} \mathrm{Si}_{1.5} \mathrm{H}_{\mathrm{y}}$ family of hydrides for several values of the parameter $y$; note the similar shape of the adiabatic temperature change for each alloy that is shifted in temperature.

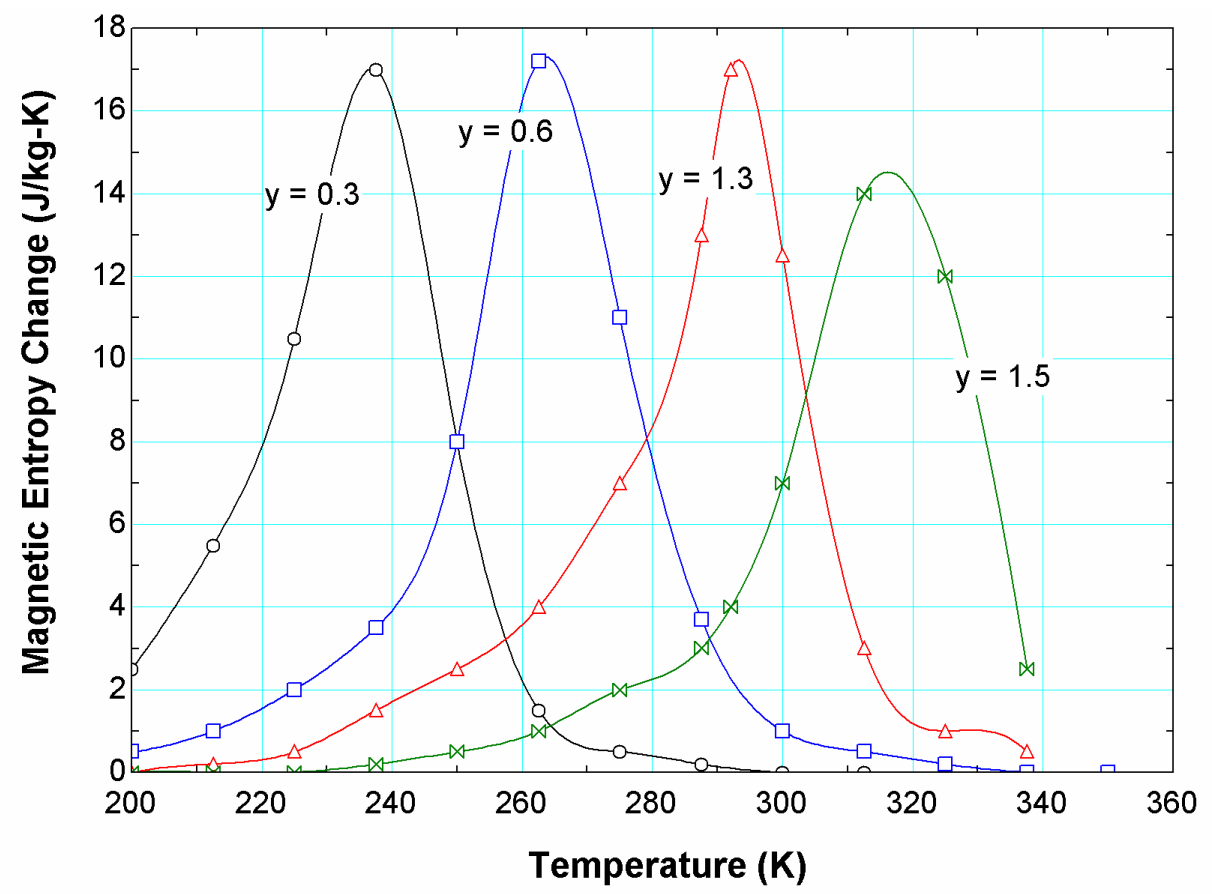

Figure 1.12. Magnetic entropy change for the $\mathrm{LaFe}_{11.5} \mathrm{Si}_{1.5} \mathrm{H}_{\mathrm{y}}$ for $\mathrm{y}=1.5,1.3,0.6$, and 0.3 (0-5 Tesla applied field swing)

A layered regenerator bed is one that consists of more than one type of magnetocaloric material. Using the new families of tunable materials a bed can be manufactured from layers of several materials each of which is placed at the optimal location in the regenerator. For example, a bed might consist of two or more materials that are chosen 
with Curie temperatures that are near the local average temperature in the regenerator so that they operate near their maximum magnetocaloric effect. Layering a regenerator bed allows an AMRR to produce a larger cooling load for a given amount of solid refrigerant when compared to a single-material bed.

\subsection{Project Objectives}

In a first order analysis, the theoretical limit of the efficiency (or COP) of an active magnetic regenerator refrigeration (AMRR) system is, essentially, the Carnot efficiency and the temperature lift of such a system is limited by the material properties of the magnetocaloric alloy. Practical limits to the performance of an AMRR have yet to be established as there is currently no directly applicable, non-proprietary model that considers the detailed, coupled thermal-fluid processes occurring within a layered magnetic regenerator bed.

The potential implementation of near room temperature AMRR systems appears to be practical in the near-term. A rotary bed configuration has been developed and demonstrated, achieving moderate levels of COP and utilizing affordable permanent magnet materials. Concurrently, the development of magnetic materials with "tunable" Curie temperatures allow layered magnetic regenerator beds to be fabricated that exhibit a large magnetocaloric effect over a large temperature range. The practical limit to the efficiency of an AMRR has not yet been firmly established, primarily because no 
adequate model is currently available for predicting the behavior of a layered bed undergoing temporally varying magnetic fields and subjected to oscillating fluid flow.

The primary focus of the effort of this project has been to develop a finite difference model of a layered regenerator. The model can account for the magnetocaloric properties of the layered bed by allowing the Curie temperature of the material in the bed to be an arbitrary function of axial position, thereby reflecting a layered bed in which the composition of the magnetic material is spatially adjusted. The bed's geometry is captured using a hydraulic diameter, porosity, and specific surface area. Correlations for the thermal and hydraulic performance and axial conduction of the matrix can be implemented for common regenerator configurations (e.g., spheres, screens, and parallel plates). The governing equations, conservation of energy applied to the magnetic material and heat transfer medium and the heat transfer rate equation, are discretized and linearized in the model. The resulting set of algebraic equations is solved for the spatial and temporal distribution of fluid and matrix temperatures using sparse matrix decomposition, subject to periodic time-varying boundary conditions on the fluid inlet temperature and flow direction, an imposed temporal applied field distribution, and the requirement of cyclic steady state. An iterative calculation process is used to determine the final, cyclic steady state condition which occurs when the linearized coefficients remain unchanged between iterations. Pressure loss through the bed and pumping power are post-calculated. 
The regenerator bed model was developed in MATLAB. Realistic models of the refrigeration system hardware and power conversion efficiencies are implemented in the MATLAB environment. The simulation tool was used to carry out a detailed study of two particular applications: a 3-ton space cooling unit and a $500 \mathrm{Btu} / \mathrm{hr}$ refrigeration unit. Optimal configurations were identified for these applications and simulations at industry standard rating conditions were carried out in order to compare the performance of an AMRR system to existing vapor compression systems. Critical modeling risk areas in the simulation tool were identified; these include both those assumptions that have the greatest impact on the performance and those with the highest uncertainty. The uncertainty in the simulation results due to these risk areas was determined. These topics are discussed in the following chapters. 


\section{Chapter 2 Numerical Model of Regenerator Bed}

\subsection{Governing Equations}

In this section, the layered bed AMR problem is described and the details of the numerical model are presented. The problem description and solution is purposefully not specific with regard to geometry, material, and fluid in order to maximize the flexibility of the numerical tool. Figure 2.1 illustrates the concept of an active magnetic regenerator modeled using a one dimensional (1D) approximation. The equipment outside of the bed, including the pumps, heat exchangers, and permanent magnets, are not explicitly modeled. Rather, their effect on the bed is felt through an imposed time variation of the mass flow rate $(\dot{m}(t))$ and the variation of the magnetic field in time and space $\left(\mu_{o} H(x, t)\right)$; these variations must somehow be related to the fluid-mechanical-magnetic configuration that is employed to operate the bed. The interface between these boundary conditions for this regenerator model and these auxiliary pieces of equipment is handled by a system-level model that interacts with this component level model. 


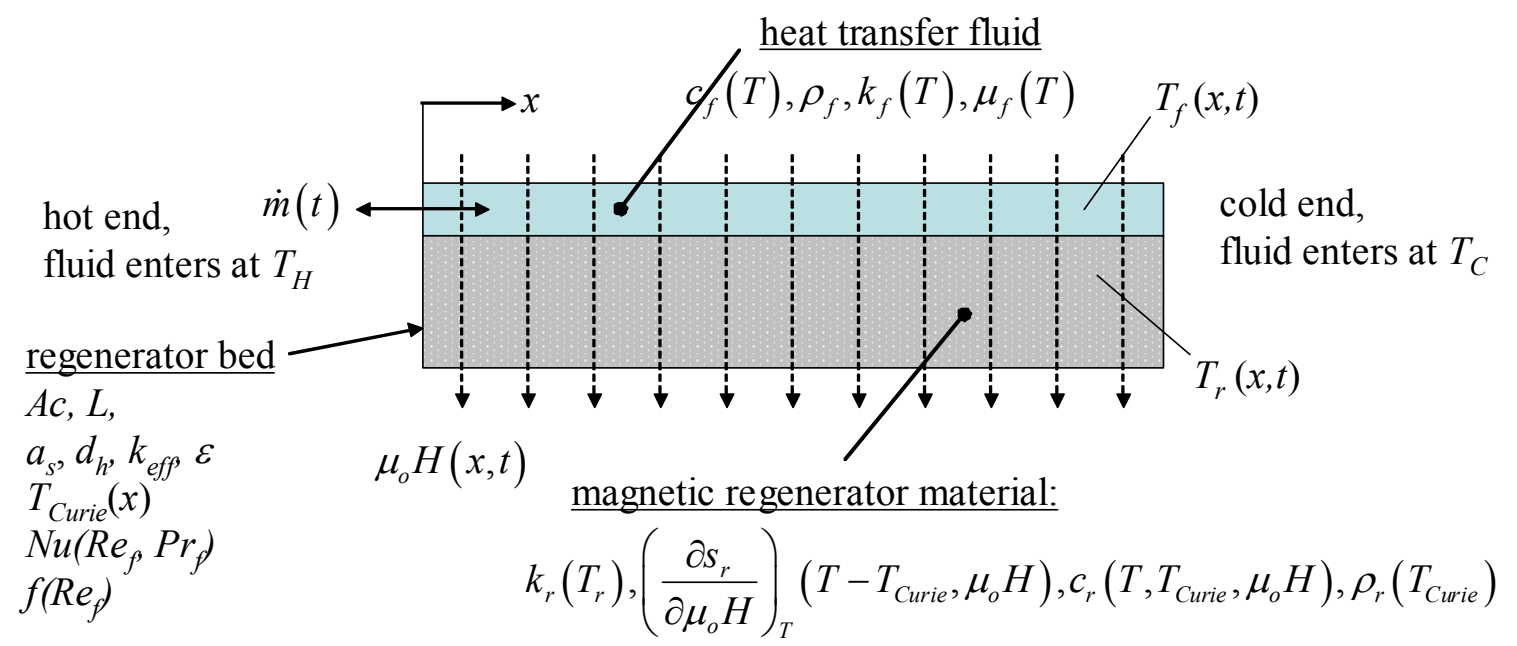

Figure 2.1. Conceptual drawing of a $1 D$ AMR model showing the important parameters

When the fluid mass flow rate is positive, flow is in the positive $x$ direction shown in Figure 2.1 and therefore enters the hot end of the regenerator bed; when it is negative it enters at the cold end. The fluid is assumed to be incompressible and therefore there can be no time variation in the mass of fluid entrained in the bed; consequently, the mass flow rate must be spatially uniform. The flow entering the bed is assumed to have the temperature of the adjacent thermal reservoir, $T_{H}$ or $T_{C}$ depending on whether the flow rate is positive or negative, respectively. The required fluid properties include the density $\left(\rho_{f}\right)$, specific heat capacity $\left(c_{f}\right)$, viscosity $\left(\mu_{f}\right)$, and thermal conductivity $\left(k_{f}\right)$. The specific heat capacity, viscosity, and thermal conductivity are assumed to be some function of temperature but not pressure. The fluid is assumed to be incompressible and so its density is unaffected by either temperature or pressure. 
The fluid flows within a regenerator matrix consisting of a magnetic material. The magnetic material may be layered; this layering is represented as a spatial variation in the Curie temperature $\left(T_{\text {Curie }}(x)\right)$. The partial derivative of the material's entropy with respect to applied field at constant temperature is a function of the temperature of the material relative to the local Curie temperature and of the applied magnetic field $\left(\frac{\partial s_{r}}{\partial \mu_{o} H_{T}}\left(T-T_{\text {Curie }}, \mu_{o} H\right)\right)$; this dependence implies that the magnetic effect of different alloys can be collapsed onto a common shape by shifting the temperature scale according to the Curie temperature which simplifies the magnetic property calculations. The specific heat capacity of the material at constant, zero applied field and the thermal conductivity of the material are assumed to be a function of the material's temperature, applied field, and its Curie temperature $\left(c_{\mu_{o} H}\left(T, T_{\text {Curie }}, \mu_{o} H\right)\right.$ and $\left.k_{r}\left(T, T_{\text {Curie }}, \mu_{o} H\right)\right)$. The material is assumed to be incompressible and therefore its density is assumed to be only a function of its Curie temperature $\left(\rho_{r}\left(T_{\text {Curie }}\right)\right)$ and not of the local temperature or applied field.

The geometry of the matrix must consist of many small passages that allow the fluid to flow in intimate thermal contact with the regenerator material. Regenerator geometries ranging from packed beds of spheres to screens to perforated plates may all be considered by this model. In order to maintain this flexibility, the regenerator geometry is characterized by a hydraulic diameter $\left(d_{h}\right)$, porosity $(\varepsilon)$, and specific surface area $\left(a_{s}\right)$. The Nusselt number of the matrix is assumed to be a function of the local Reynolds 
number and Prandtl number of the fluid $\left(N u\left(\operatorname{Re}_{f}, \operatorname{Pr}_{f}\right)\right)$. The friction factor is assumed to be a function of the local Reynolds number $\left(f\left(R e_{f}\right)\right)$. The matrix is also assumed to be characterized by an effective thermal conductivity $\left(k_{\text {eff }}\right)$ that relates the actual, axial conduction heat transfer to the heat transfer through a comparable solid piece of material. The above constraints will be specified more completely based on the particular geometry and materials that are selected. The overall size of the regenerator is specified according to its length $(L)$ and total cross-sectional area $\left(A_{c}\right)$.

The fluid and regenerator temperature variations over a steady-state cycle are ultimately the output of this model $\left(T_{f}(x, t)\right.$ and $\left.T_{r}(x, t)\right)$. These variations, coupled with the prescribed mass flow rate will allow the calculation of cycle performance metrics such as refrigeration load and the magnetic power required. The temperature variations are obtained by solving a set of coupled, partial differential equations in time and space that are obtained from $1^{\text {st }}$ law considerations on the fluid and the matrix. Figure 2.2 illustrates a differential segment of the fluid with the various energy flows indicated. 


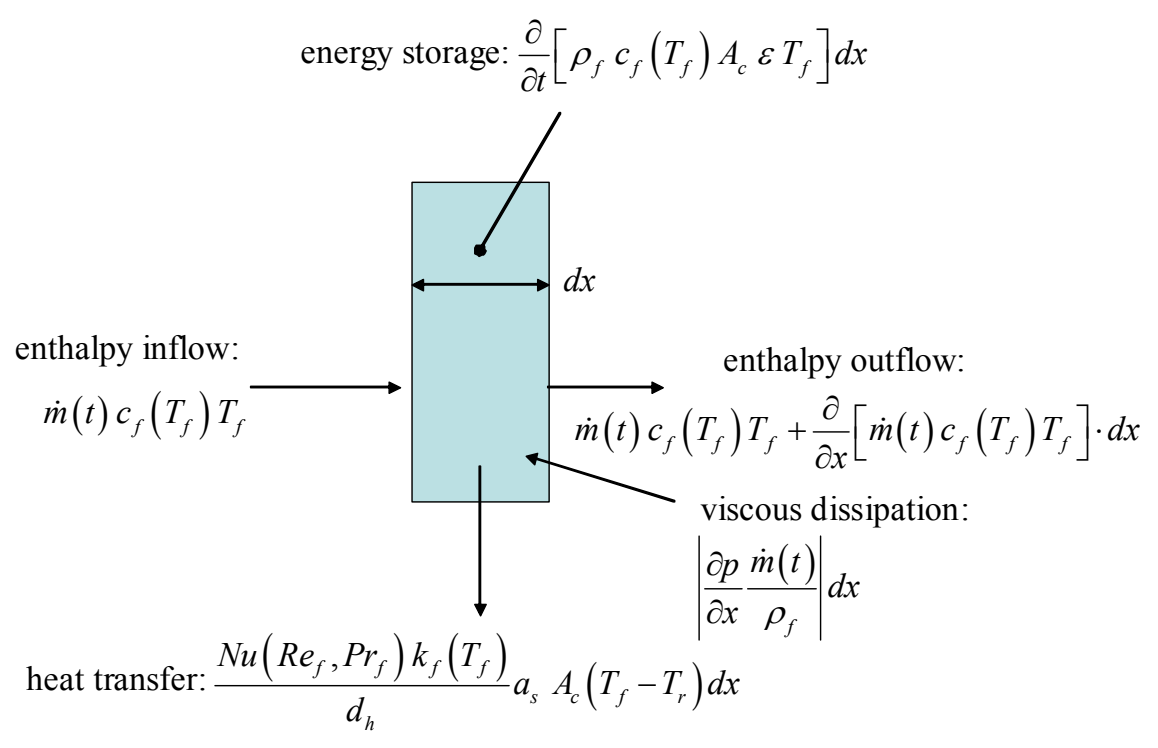

Figure 2.2. Differential segment of entrained fluid with energy terms indicated

After some simplification, the energy balance on the fluid suggested by Fig. 2.2 is:

$$
\dot{m}(t) \frac{\partial}{\partial x}\left[c_{f}\left(T_{f}\right) T_{f}\right]+\frac{N u\left(R e_{f}, P r_{f}\right) k_{f}\left(T_{f}\right)}{d_{h}} a_{s} A_{c}\left(T_{f}-T_{r}\right)+\rho_{f} A_{c} \varepsilon \frac{\partial}{\partial t}\left[c_{f}\left(T_{f}\right) T_{f}\right]=\left|\frac{\partial p}{\partial x} \frac{\dot{m}(t)}{\rho_{f}}\right|
$$

Note that axial conduction through the fluid is neglected. The axial conduction in the AMR is split between the fluid and the bed; the amount of conduction in the fluid may be non-negligible due to its relatively high thermal conductivity and the effects of fluid dispersion. However, the axial conduction is applied to the matrix and modeled using the concept of an effective bed conductivity. The third term in Equation (2.1) represents the heat capacity of the fluid entrained in the matrix. It simplifies the governing equations and stabilizes the numerical solution considerably if this term is included with the heat capacity of the matrix itself; therefore this term is removed from the fluid energy equation but it will be included in the regenerator energy equation. This approximation 
will underpredict the performance of the regenerator and therefore a method to correct for this assumption during periods of fluid flow is described in section 2.6. The approximation, even with the correction, will fail to account for losses related to the heat transfer between the bed and the fluid required during the zero- or low-flow periods of the cycle where the bed temperature is changing and the fluid temperature lags. The final term represents viscous dissipation in the fluid.

After expanding the derivatives in Equation (2.1) and substituting the friction factor for the pressure gradient, it becomes:

$$
\dot{m}(t)\left(c_{f}\left(T_{f}\right)+T_{f} \frac{\partial c_{f}\left(T_{f}\right)}{\partial T}\right) \frac{\partial T_{f}}{\partial x}+\frac{N u\left(R e_{f}, P r_{f}\right) k_{f}\left(T_{f}\right)}{d_{h}} a_{s} A_{c}\left(T_{f}-T_{r}\right)=\left|\frac{f_{f}\left(R e_{f}\right) \dot{m}(t)^{3}}{2 \rho_{f}^{2} A_{c}^{2} d_{h}}\right|
$$

The product of temperature and the partial derivative of the specific heat capacity with temperature in the $1^{\text {st }}$ term is much smaller than the specific heat capacity itself for any practical heat transfer fluid and therefore Eq. (2.2) can be written as:

$$
\dot{m}(t) c_{f}\left(T_{f}\right) \frac{\partial T_{f}}{\partial x}+\frac{N u\left(\operatorname{Re}_{f}, \operatorname{Pr}_{f}\right) k_{f}\left(T_{f}\right)}{d_{h}} a_{s} A_{c}\left(T_{f}-T_{r}\right)=\left|\frac{f_{f}\left(\operatorname{Re}_{f}\right) \dot{m}(t)^{3}}{2 \rho_{f}^{2} A_{c}^{2} d_{h}}\right|
$$

Figure 2.3 illustrates a differential segment of the regenerator material with the various energy flows indicated: 


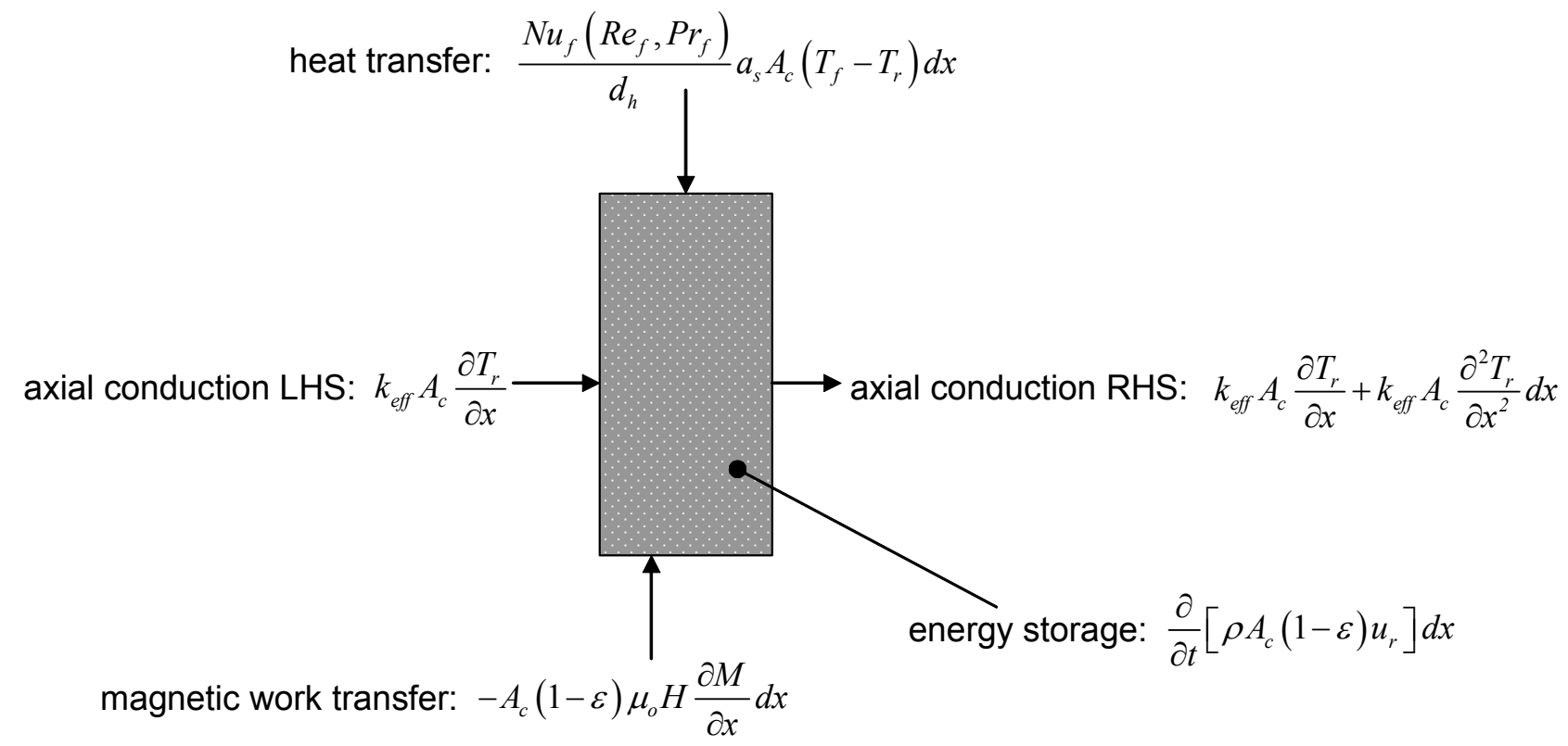

Figure 2.3. Differential segment of regenerator with energy terms indicated

The energy balance suggested by Figure 2.3 is:

$$
\frac{N u\left(R e_{f}, P r_{f}\right) k_{f}\left(T_{f}\right)}{d_{h}} a_{s} A_{s}\left(T_{f}-T_{r}\right)+A_{c}(1-\varepsilon) \mu_{o} H \frac{\partial M}{\partial t}+k_{e f f} A_{c} \frac{\partial^{2} T_{r}}{\partial x^{2}}=\rho_{r} A_{c}(1-\varepsilon) \frac{\partial u_{r}}{\partial t}
$$

The heat capacity of the fluid is added to Eq. (2.4); however, the fluid is assumed to be at the regenerator temperature. The magnetic work term is grouped with the internal energy to obtain:

$$
\begin{aligned}
& \frac{N u\left(R e_{f}, P r_{f}\right) k_{f}\left(T_{f}\right)}{d_{h}} a_{s} A_{s}\left(T_{f}-T_{r}\right)+k_{e f f} A_{c} \frac{\partial^{2} T_{r}}{\partial x^{2}}= \\
& A_{c}(1-\varepsilon) \rho_{r}\left(\frac{\partial u_{r}}{\partial t}-\mu_{o} H \frac{\partial\left(v_{r} M\right)}{\partial t}\right)+\rho_{f} A_{c} \varepsilon \frac{\partial}{\partial t}\left[c_{f}\left(T_{r}\right) T_{r}\right]
\end{aligned}
$$


As with the fluid energy balance, the specific heat capacity is pulled out of the partial derivative. The first term on the right hand side of Equation (2.5) is the sum of a differential change in internal energy and a differential work transfer; this must be equal to a differential heat transfer, which is related to a change in entropy. Therefore, Equation (2.5) may be rewritten according to:

$$
\frac{N u\left(R e_{f}, P r_{f}\right) k_{f}\left(T_{f}\right)}{d_{h}} a_{s} A_{s}\left(T_{f}-T_{r}\right)+k_{e f f} A_{c} \frac{\partial^{2} T_{r}}{\partial x^{2}}=A_{c}(1-\varepsilon) \rho_{r} T_{r} \frac{\partial s_{r}}{\partial t}+\rho_{f} A_{c} \varepsilon c_{f}\left(T_{r}\right) \frac{\partial T_{r}}{\partial t}
$$

The change in regenerator entropy is divided into a temperature driven and a magnetic driven component to yield the final, regenerator governing equation:

$$
\begin{aligned}
& \frac{N u\left(R e_{f}, P r_{f}\right) k_{f}\left(T_{f}\right)}{d_{h}} a_{s} A_{s}\left(T_{f}-T_{r}\right)+k_{e f f} A_{c} \frac{\partial^{2} T_{r}}{\partial x^{2}}= \\
& A_{c}(1-\varepsilon) \rho_{r} T_{r} \frac{\partial s_{r}}{\partial \mu_{o} H}\left(T_{r}-T_{\text {Curie }}, \mu_{o} H\right) \frac{\partial \mu_{o} H}{\partial t} \\
& +A_{c}\left[\rho_{f} \varepsilon c_{f}\left(T_{r}\right)+(1-\varepsilon) \rho_{r} c_{\mu_{o} H}\left(T_{r}, T_{\text {Curie }}, \mu_{o} H\right)\right] \frac{\partial T_{r}}{\partial t}
\end{aligned}
$$

The fluid is assumed to enter the matrix at the temperature of the associated heat exchanger, providing the required spatial boundary conditions:

$$
\begin{aligned}
& \text { if } \dot{m}(t) \geq 0 \text { then } T_{f}(x=0, t)=T_{H} \\
& \text { if } \dot{m}(t)<0 \text { then } T_{f}(x=L, t)=T_{C}
\end{aligned}
$$

The regenerator must undergo a steady-state cycle, leading to the required temporal boundary condition:

$$
T_{r}(x, t=0)=T_{r}(x, t=\tau)
$$


where $\tau$ is the duration of a cycle.

The numerical solution for the fluid temperature is obtained over a grid that extends from 0 to $L$ in space and from 0 to $\tau$ in time, as shown in Figure 2.4.

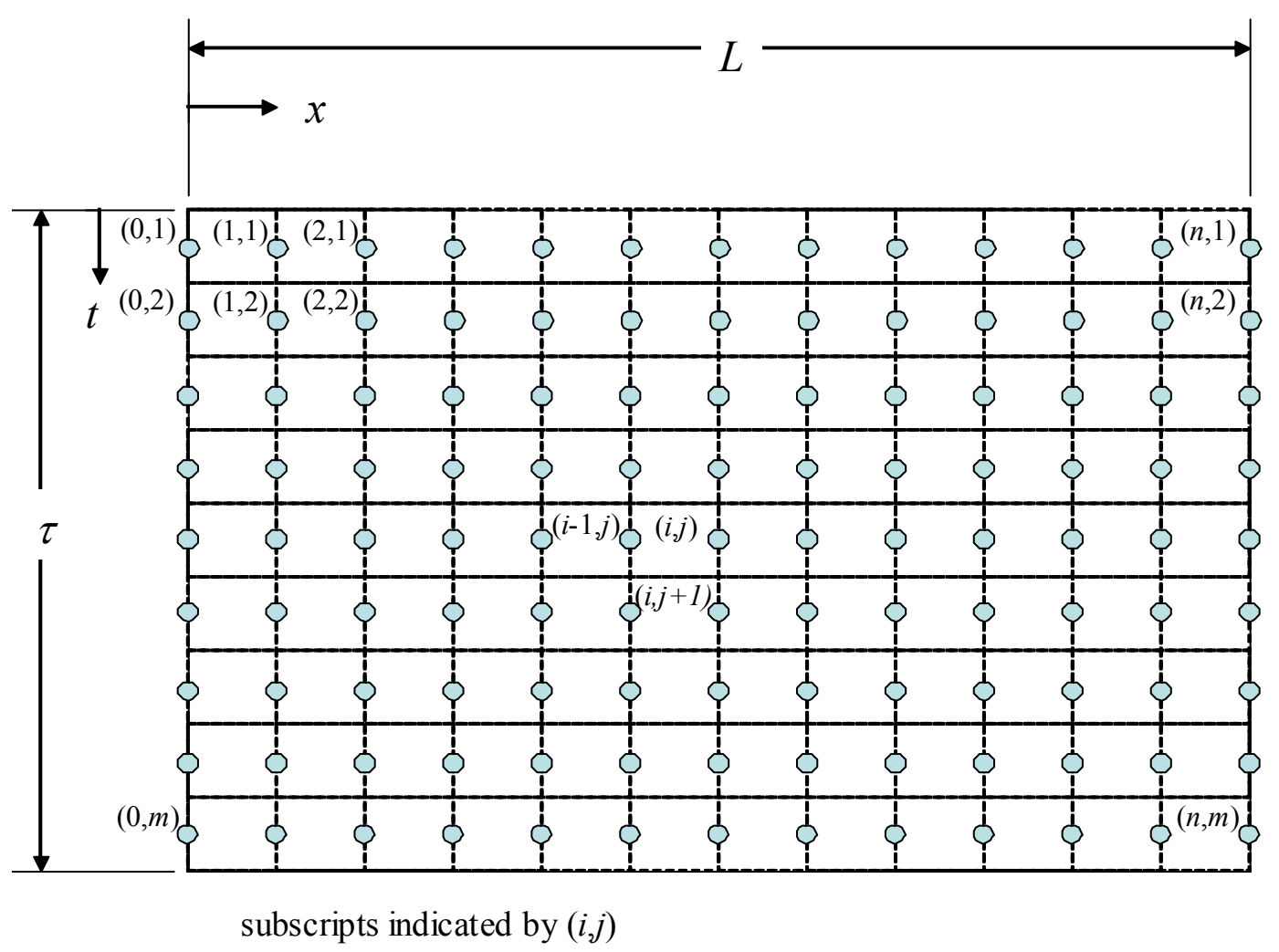

Figure 2.4. Numerical grid used for fluid temperature solution

The axial location of each fluid temperature node $\left(x_{f i}\right)$ is given by:

$$
x_{f i}=i \frac{L}{n} \quad i=0 . . n
$$


where $i$ is the axial subscript and $n$ is the total number of axial control volumes that are used. The time associated with each fluid temperature node $\left(t_{f j}\right)$ is given by:

$$
t_{f j}=\left(j-\frac{1}{2}\right) \frac{\tau}{m} j=1 . . m
$$

The numerical solution for the regenerator temperature is obtained over a numerical grid shown in Figure 2.5.

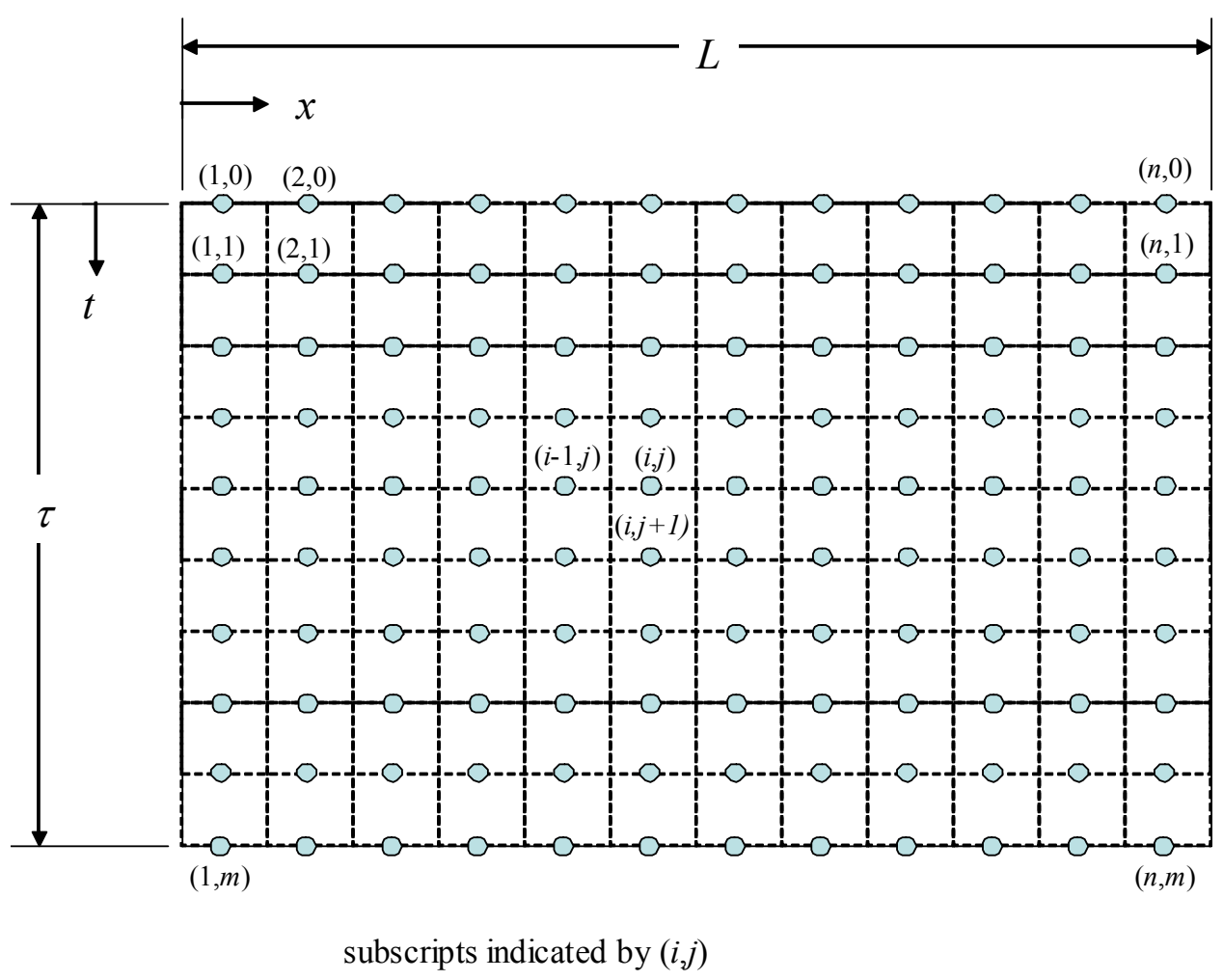

Figure 2.5. Numerical grid used for regenerator temperature solution

The axial location of each regenerator temperature node $\left(x_{r}\right)$ is given by:

$$
x_{r i}=\left(i-\frac{1}{2}\right) \frac{L}{n} \quad i=1 . . n
$$


where $i$ is the axial subscript and $n$ is the total number of axial control volumes that are used. The time associated with each fluid temperature node $\left(t_{f j}\right)$ is given by:

$$
t_{f j}=j \frac{\tau}{m} j=0 . . m
$$

Initially, "guess" values for the temperatures at each node $\left(T_{r i, j}^{*}\right.$ and $\left.T_{f i, j}^{*}\right)$ are assigned based on a spatially linear and time invariant assumption.

$$
\begin{aligned}
& T_{r i, j}^{*}=T_{H}-\frac{x_{r i}}{L}\left(T_{H}-T_{C}\right) i=1 . . n j=0 . . m \\
& T_{f i, j}^{*}=T_{H}-\frac{x_{f i}}{L}\left(T_{H}-T_{C}\right) i=0 . . n j=1 . . m
\end{aligned}
$$

The fluid energy balance is discretized and written for each control volume. The energy balances differ according to the direction of the mass flow rate:

if $\dot{m}\left(t_{f j}\right)>0$ then $N u_{i, j}^{*} k_{f i, j}^{*} \frac{a_{s} A_{c}}{d_{h}}\left(\frac{T_{f i, j}+T_{f i-1, j}}{2}-\frac{T_{r i, j}+T_{r i, j-1}}{2}\right)+\dot{m}\left(t_{f j}\right) c_{f i, j}^{*} \frac{\left(T_{f i, j}-T_{f i-1, j}\right) n}{L}=\left|\frac{f_{f i, j}^{*} \dot{m}\left(t_{f, j}\right)^{3}}{2 \rho_{f}^{2} A_{c}^{2} d_{h}}\right|$ $i=1 . . n \quad j=1 . . m$ 
if $\dot{m}\left(t_{f j}\right)>0$ then

$N u_{i, j}^{*} k_{f i, j}^{*} \frac{a_{s} A_{c}}{d_{h}}\left(\frac{T_{f i, j}+T_{f i-1, j}}{2}-\frac{T_{r i, j}+T_{r i, j-1}}{2}\right)+\dot{m}\left(t_{f j}\right) c_{f i, j}^{*} \frac{\left(T_{f i, j}-T_{f i-1, j}\right) n}{L}=\left|\frac{f_{f i, j}^{*} \dot{m}\left(t_{f, j}\right)^{3}}{2 \rho_{f}^{2} A_{c}^{2} d_{h}}\right|$

$i=1 . . n \quad j=1 . . m$

where $N u_{f i, j}^{*}$ is the Nusselt number based on the guess fluid temperature values in the node,

$$
N u_{f i, j}^{*}=N u\left(\operatorname{Re}_{f i, j}^{*}, \operatorname{Pr}_{f i, j}^{*}\right)
$$

$R e_{f}^{*}$ is the Reynolds number for the fluid computed using the "guess" fluid temperature and the free flow velocity and $\operatorname{Pr}_{f i, j}^{*}$ is the Prandtl number of the fluid, also computed using the "guess" fluid temperature:

$$
\begin{aligned}
& \operatorname{Re}_{f i, j}^{*}=\frac{d_{h} \dot{m}\left(t_{f j}\right)}{A_{c} \mu_{f i, j}^{*}} \\
& \operatorname{Pr}_{f i, j}^{*}=\frac{c_{f i, j}^{*} \mu_{f i, j}^{*}}{k_{f i, j}^{*}}
\end{aligned}
$$

The friction factor $\left(f_{f i, j}^{*}\right)$ in Equation (2.16) is also based on the guess fluid temperatures within the node:

$$
f_{f i, j}^{*}=f_{f}\left(\operatorname{Re}_{f i, j}^{*}\right)
$$


The fluid properties in equations (2.18) through (2.20) are based on the average temperature in the time and space occupied by the control volume. Collecting like terms in equations (2.16a) and (2.16b) leads to:

$$
\begin{aligned}
& \text { if } \dot{m}\left(t_{f j}\right)>0 \text { then } \\
& T_{f i, j}\left[N u_{f i, j}^{*} k_{f i, j}^{*} \frac{a_{s} A_{c}}{2 d_{h}}+\dot{m}\left(t_{f j}\right) c_{f i, j}^{*} \frac{n}{L}\right]+T_{f i-1, j}\left[N u_{f i, j}^{*} k_{f i, j}^{*} \frac{a_{s} A_{c}}{2 d_{h}}-\dot{m}\left(t_{f j}\right) c_{f i, j}^{*} \frac{n}{L}\right] \\
& +T_{r i, j}\left[-N u_{f i, j}^{*} k_{f i, j}^{*} \frac{a_{s} A_{c}}{2 d_{h}}\right]+T_{r i, j-1}\left[-N u_{f i, j}^{*} k_{f i, j}^{*} \frac{a_{s} A_{c}}{2 d_{h}}\right]=\left|\frac{f_{i, j}^{*} \dot{m}\left(t_{f, j}\right)^{3}}{2 \rho_{f}^{2} A_{c}^{2} d_{h}}\right| \\
& i=1 . . n j=1 . . m
\end{aligned}
$$

$$
\begin{aligned}
& \text { if } \dot{m}\left(t_{f j}\right)<0 \text { then } \\
& T_{f i, j}\left[N u_{f i, j}^{*} k_{f i, j}^{*} \frac{a_{s} A_{c}}{2 d_{h}}-\dot{m}\left(t_{f j}\right) c_{f i, j}^{*} \frac{n}{L}\right]+T_{f i+1, j}\left[N u_{f i, j}^{*} k_{f i, j}^{*} \frac{a_{s} A_{c}}{2 d_{h}}+\dot{m}\left(t_{f j}\right) c_{f i, j}^{*} \frac{n}{L}\right] \\
& +T_{r i+1, j}\left[-N u_{f i, j}^{*} k_{f i, j}^{*} \frac{a_{s} A_{c}}{2 d_{h}}\right]+T_{r i+1, j-1}\left[-N u_{f i, j}^{*} k_{f i, j}^{*} \frac{a_{s} A_{c}}{2 d_{h}}\right]=\left|\frac{f_{i, j}^{*} \dot{m}\left(t_{f, j}\right)^{3}}{2 \rho_{f}^{2} A_{c}^{2} d_{h}}\right| \\
& i=0 . .(n-1) j=1 . . m
\end{aligned}
$$

The boundary conditions for the fluid temperatures are specified by:

$$
\begin{aligned}
& \text { if } \dot{m}\left(t_{f j}\right) \geq 0 \text { then } T_{f 0, j}[1]=T_{H} \\
& \text { if } \dot{m}\left(t_{f j}\right)<0 \text { then } T_{f n, j}[1]=T_{C} \\
& j=1 . . m
\end{aligned}
$$

The regenerator energy balances are likewise discretized and written for each control volume: 


$$
\begin{aligned}
& N u_{f i, j}^{*} k_{f i, j}^{*} \frac{a_{s} A_{s}}{d_{h}}\left(\frac{T_{r i, j}+T_{r i, j-1}}{2}-\frac{\left.T_{f i, j}+T_{f i-1, j}\right)}{2}\right) \\
& +A_{c}\left[\rho_{f} \varepsilon c_{f i, j}^{*}+(1-\varepsilon) \rho_{r} c_{\mu_{o} H i, j}^{*} \frac{\left(T_{r i, j}-T_{r i, j-1}\right) m}{\tau}\right. \\
& +\frac{n^{2} k_{e f f i, j}^{*} A_{c}}{L^{2}}\left[\frac{\left(T_{r i, j-1}+T_{r i, j}\right)}{2}-\frac{\left(T_{r i-1, j-1}+T_{r i-1, j}\right)}{2}\right] \\
& +\frac{n^{2} k_{e f f i, j}^{*} A_{c}}{L^{2}}\left[\frac{\left(T_{r i, j-1}+T_{r i, j}\right)}{2}-\frac{\left(T_{r i+1, j-1}+T_{r i+1, j}\right)}{2}\right]= \\
& -A_{c}(1-\varepsilon) \rho_{r} \frac{\left(T_{r i, j}^{*}+T_{r i, j-1}^{*}\right)}{2}\left(\frac{\partial s_{r}}{\partial \mu_{o} H}\right)_{i, j}^{*} \frac{\partial \mu_{o} H}{\partial t}\left(x_{r i}, \frac{t_{r j}+t_{r j-1}}{2}\right) \\
& i=1 . . n j=1 . . m
\end{aligned}
$$

where $c_{\mu_{o} H i, j}^{*}$ and $\left(\frac{\partial s}{\partial \mu_{o} H}\right)_{i, j}^{*}$ are the magnetic properties evaluated at the average guess value for the regenerator over the temporal and spatial extent of the control volume and at the local value of the magnetic field and the Curie temperature. Note that the $3^{\text {rd }}$ and $4^{\text {th }}$ terms in Eq. (2.23) represent conduction to the control neighboring control volumes on the left- and right-hand sides, respectively, and therefore are eliminated at the warm and cold ends, respectively. Collecting like terms leads to: 


$$
\begin{aligned}
& T_{r i, j}\left[N u_{i, j}^{*} k_{f i, j}^{*} \frac{a_{s} A_{s}}{2 d_{h}}+A_{c}\left[\rho_{f} \varepsilon c_{f i, j}^{*}+(1-\varepsilon) \rho_{r} c_{\mu_{o} H i, j}^{*}\right] \frac{m}{\tau}+\frac{n^{2} k_{e f f i, j}^{*} A_{c}}{L^{2}}\right] \\
& +T_{r i, j-1}\left[N u_{i, j}^{*} k_{f i, j}^{*} \frac{a_{s} A_{s}}{2 d_{h}}-A_{c}\left[\rho_{f} \varepsilon c_{f i, j}^{*}+(1-\varepsilon) \rho_{r} c_{\mu_{o} H i, j}^{*}\right] \frac{m}{\tau}+\frac{n^{2} k_{e f f i, j}^{*} A_{c}}{L^{2}}\right] \\
& +T_{r i-1, j-1}\left[-\frac{n^{2} k_{e f f i, j}^{*} A_{c}}{2 L^{2}}\right]+T_{r i-1, j}\left[-\frac{n^{2} k_{e f f i, j}^{*} A_{c}}{2 L^{2}}\right]+T_{r i+1, j-1}\left[-\frac{n^{2} k_{e f f i, j}^{*} A_{c}}{2 L^{2}}\right]+T_{r i+1, j}\left[-\frac{n^{2} k_{e f f i, j}^{*} A_{c}}{2 L^{2}}\right] \\
& +T_{f i, j}\left[-N u_{i, j}^{*} k_{f i, j}^{*} \frac{a_{s} A_{s}}{2 d_{h}}\right]+T_{f i-1, j}\left[-N u_{i, j}^{*} k_{f i, j}^{*} \frac{a_{s} A_{s}}{2 d_{h}}\right] \\
& =-A_{c}(1-\varepsilon) \rho_{r} \frac{\left(T_{r i, j}^{*}+T_{r i, j-1}^{*}\right)}{2}\left(\frac{\partial s_{r}}{\partial \mu_{o} H}\right)_{i, j}^{*} \frac{\partial \mu_{o} H}{\partial t}\left(x_{r i}, \frac{t_{r j}+t_{r j-1}}{2}\right) \\
& i=1 . . n j=1 . . m
\end{aligned}
$$

The boundary condition for the regenerator temperature distribution is expressed by:

$$
T_{r i, 0}[1]+T_{r i, m}[-1]=0 \quad i=1 . . n
$$

\subsection{Numerical Solution Algorithm}

Equations (2.21), (2.22), (2.24), and (2.25) form a system of linear equations in each of the nodal temperatures shown in Figures 2.4 and 2.5 for the regenerator and fluid. These equations are solved using a sparse matrix decomposition algorithm. The absolute value of the maximum error between the "guess" values of the regenerator and fluid temperatures and the calculated values is determined at each iteration. If the error is less than a relaxation tolerance $(t o l)$ then the relaxation process is complete. If not, then a new set of guess values are used in a subsequent iteration. These new "guess" values $\left(T^{*+}\right)$ are computed as the weighted average of the calculated and "guess" values. 


$$
\begin{aligned}
& T_{f i, j}^{*_{+}}=w t T_{f i, j}+(1-w t) T_{f i, j}^{*} \quad i=0 . . n j=1 . . m \\
& T_{r i, j}^{*_{+}}=w t T_{r i, j}+(1-w t) T_{r i, j}^{*} i=1 . . n j=0 . . m
\end{aligned}
$$

The numerical model in its current MATLAB implementation is listed as Appendix A.

The assumptions used to derive the numerical model were described as the model was derived in the previous section and are summarized below:

- the heat transfer fluid is incompressible; therefore the mass flow rate does not vary spatially within the matrix and the mass of fluid entrained in the matrix is constant,

- the "shape" of the magnetic entropy variation must be normalized against the Curie temperature of the alloy; this allows the layering of the bed to be captured by the distribution of the Curie temperature of the bed material,

- the bed geometry is uniform; no spatial gradients exist in the bed characteristics such as hydraulic diameter, porosity, etc.,

- the fluid flow is one-dimensional; the flow maldistribution effects are neglected,

- the magnetization and demagnetization processes are assumed to be internally reversible with no hysteresis or temperature gradients,

- the thermal capacity of the fluid entrained in the matrix is lumped together with the thermal capacity of the matrix itself; this implies that the time derivatives of the fluid and regenerator temperatures are of similar magnitude which is a good assumption provided that the local temperature difference between the fluid and regenerator is small in comparison with the overall temperature range spanned by 
the regenerator; note that this assumption is at least approximately relaxed by correcting for the entrained fluid capacity, as described in a subsequent section,

- the product of temperature and the partial derivative of the fluid heat capacity with temperature is assumed to be much less than the specific heat capacity itself; therefore, the terms in the energy equation associated with the fluid flow and fluid energy storage are related only to the local specific heat capacity,

\subsection{Verification of Model}

There are no general analytical solutions to the regenerator equations presented in section 2.2. However, in the limit of constant properties and no axial conduction, the thermal effectiveness $(\varepsilon)$ of a conventional regenerator (i.e., one with no magnetocaloric effect) subjected to a mass flow rate variation with the shape shown in Figure 2.6 is known.

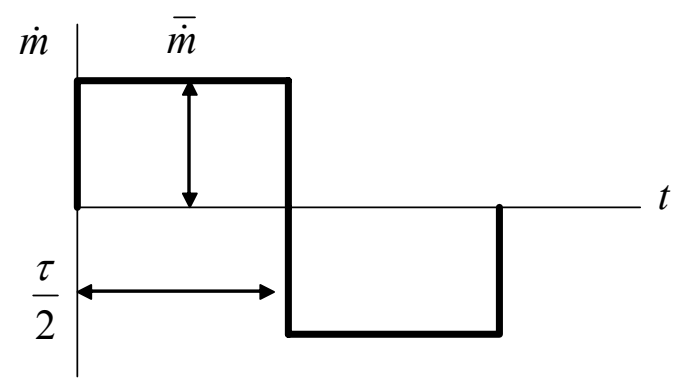

Figure 2.6. Mass flow rate variation for an idealized regenerator

The thermal effectiveness is defined as:

$$
\varepsilon_{t} \equiv \frac{\dot{q}_{f-r}}{\overline{\dot{m}} c_{f}\left(T_{H}-T_{C}\right)}
$$


where $\overline{\dot{m}}$ is the magnitude of the mass flow rate and $\dot{q}_{f-r}$ is the average heat transfer rate between the fluid and the matrix during the cycle:

$$
\dot{q}_{f-r}=\overline{\dot{m}} c_{f} \int_{0}^{\tau / 2}\left(T_{H}-T_{f}(x=L, t)\right) d t
$$

The typical variables used to characterize this problem are the number of transfer units (NTU, sometimes also referred to as the reduced length of the regenerator) and the utilization ratio ( $U$, the inverse of the matrix capacity rate ratio, Ackermann (1997)).

$$
\begin{gathered}
N T U \equiv \frac{N u k_{f} a_{s} L A_{c}}{d_{h} \overline{\dot{m}} c_{f}} \\
U=\frac{\overline{\dot{m}} c_{f} \tau}{2 A_{c} L(1-\varepsilon) \rho_{r} c_{\mu_{o} H}}
\end{gathered}
$$

Dragutinovic and Baclic (1998) present tables for the $\varepsilon_{t}$ as a function of $N T U$ and $U$ in this limit. The numerical model can be verified against these solutions by:

1. setting all fluid properties $\left(c_{f}, k_{f}, \rho_{f}\right.$, and $\left.\mu_{f}\right)$ equal to constants,

2. setting the partial derivative of entropy with respect to magnetic field equal to zero,

3. setting the remaining regenerator properties $\left(c_{\mu_{o} H}\right.$ and $\left.\rho_{r}\right)$ equal to constants,

4. setting the Nusselt number $\left(N u_{f}\right)$ equal to a constant,

5. setting the effective thermal conductivity of the matrix $\left(k_{\text {eff }}\right)$ equal to zero,

6. setting the friction factor $(f)$ equal to zero,

7. setting the bed parameters $\left(\varepsilon, a_{s}\right)$ and bed size $\left(A_{c}\right.$ and $\left.L\right)$ equal to constants, and 
8. applying the functional form of the mass flow rate shown in Figure 2.6 for a fixed cycle duration $(\tau)$

$$
\dot{m}(t)=\operatorname{sign}\left(\frac{\tau}{2}-t\right) \overline{\dot{m}}
$$

By varying the hydraulic diameter $\left(d_{h}\right)$ and mass flow rate $(\overline{\dot{m}})$, it is possible to set the $N T U$ and $U$. The numerical model was implemented under these conditions using a grid with 80 spatial control volumes $(n=80)$ and 100 time steps $(m=100)$. The results are illustrated in Figure 2.7. Notice the excellent agreement between the published and predicted results, verifying the accuracy of the numerical model in this limit.

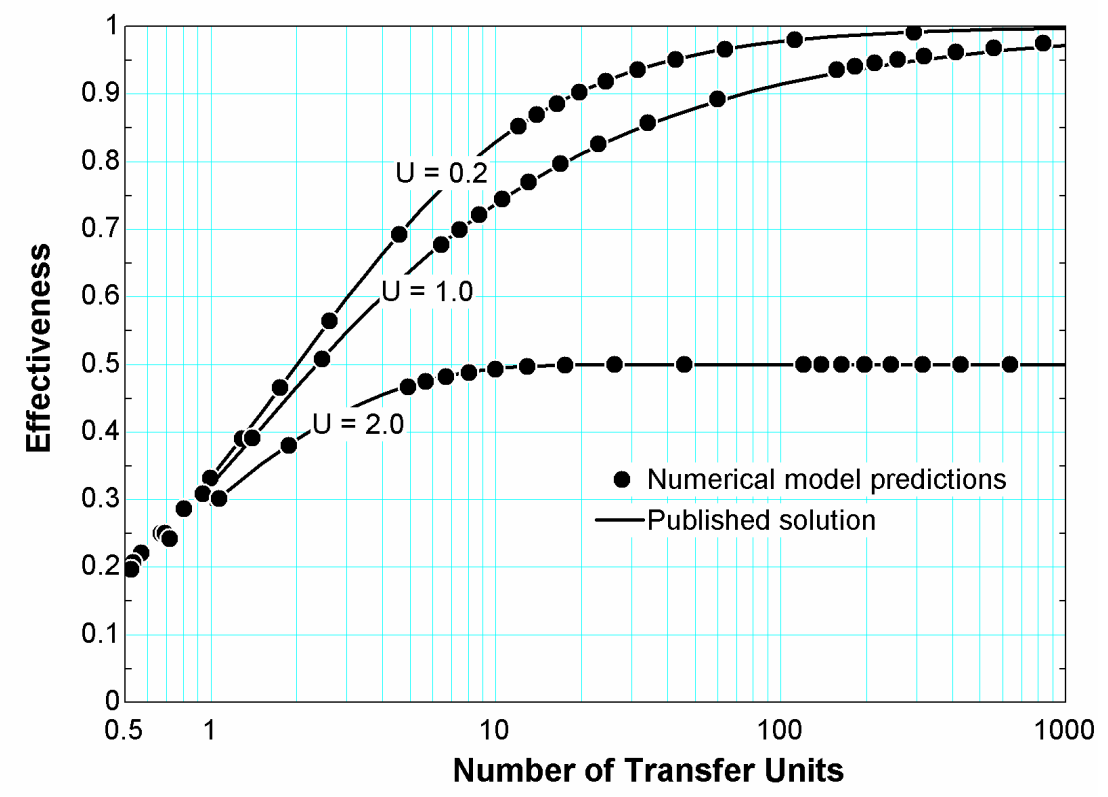

Figure 2.7. Numerical model predictions and published results for $\varepsilon_{t}$ as a function of $N T U$ and various values of $U$ in the ideal regenerator limit 


\subsection{Material Properties}

\subsubsection{Heat Transfer Fluid Properties}

The fluid properties $\mu_{f}, c_{f}, h_{f}$ and $k_{f}$ are determined within the model assuming that the fluid is incompressible. The properties are calculated within a Matlab function that takes the fluid temperature as its only input. Interpolation from a fluid property data table requires significant computational time given the iterative nature of the program. Therefore, the fluid properties are curve fit to a polynomial function of temperature. The property correlations built into EES were used to generate property data over a relevant range of temperatures. For water, the data was generated between $273.3 \mathrm{~K}$ to $372.6 \mathrm{~K}$ and then imported into MATLAB where a polynomial function was fit to each fluid property in terms of a dimensionless temperature, $T_{f} / T_{r e f}$, where $T_{r e f}$ is an arbitrarily chosen reference temperature. For water, $T_{\text {ref }}$ was chosen as $273.3 \mathrm{~K}$. The order of the polynomial was determined by using the lowest order fit that resulted in a maximum discrepancy of less than $0.5 \%$ between the fit data and the data obtained from EES. The equation for conductivity of the heat transfer fluid is

$$
k_{f}\left(T_{f}\right)=\sum_{i=0}^{N} a_{i}\left(\frac{T_{f}}{T_{\text {ref }}}\right)^{i}
$$

where $a$ is a set of curve-fit coefficients and $N$ is the order of the curve fit used.

Equations for specific heat and viscosity have identical forms. The coefficients used for each property curve fit along with the freezing point and reference temperature of each fluid investigated are shown in Tables $2.1-2.3$. 
Table 2.1. Coefficients of property functions for water

\begin{tabular}{|c|c|c|c|}
\hline fluid & water & & \\
\hline$T_{\text {solid }}$ & $273.15 \mathrm{~K}$ & & \\
\hline \multirow[t]{2}{*}{$\mathrm{T}_{\text {ref }}$} & $273.3 \mathrm{~K}$ & & \\
\hline & $k_{f}$ & $\overline{C_{f}}$ & $\mu_{f}$ \\
\hline$a_{0}$ & -0.774277 & 7526.83 & 1.803302 \\
\hline$a_{1}$ & 2.055221 & -7522.49 & -7.216117 \\
\hline$a_{2}$ & -0.733619 & 5491.12 & 11.58833 \\
\hline$a_{3}$ & & -1288.18 & -9.32406 \\
\hline$a_{4}$ & & & 3.756011 \\
\hline$a_{5}$ & & & -0.605683 \\
\hline
\end{tabular}

Table 2.2. Coefficients of property functions for ethylene glycol and water solution

\begin{tabular}{|c|c|c|c|}
\hline fluid & \multicolumn{3}{|c|}{$50 \%$ ethylene glycol, $50 \%$ water } \\
\hline $\mathrm{T}_{\text {solid }}$ & $273.15 \mathrm{~K}$ & & \\
\hline \multirow[t]{2}{*}{$\mathrm{T}_{\text {ref }}$} & $273.3 \mathrm{~K}$ & & \\
\hline & $k_{f}$ & $C_{f}$ & $\mu_{\mathrm{f}}$ \\
\hline$a_{0}$ & 0.226624 & 1874.60 & 667.5282 \\
\hline$a_{1}$ & 0.116852 & 1035.78 & -3235.9249 \\
\hline$a_{2}$ & 0.011815 & 91.88 & 6538.4483 \\
\hline$a_{3}$ & & & -7046.8471 \\
\hline$a_{4}$ & & & 4271.6129 \\
\hline$a_{5}$ & & & -1380.5999 \\
\hline$a_{6}$ & & & 185.8452 \\
\hline
\end{tabular}


Table 2.3. Coefficients of property functions for propylene glycol and water solution

\begin{tabular}{|c|c|c|c|}
\hline fluid & \multicolumn{3}{|c|}{$50 \%$ propylene glycol, $50 \%$ water } \\
\hline $\mathrm{T}_{\text {solid }}$ & $240.05 \mathrm{~K}$ & & \\
\hline \multirow[t]{2}{*}{$\mathrm{T}_{\text {ref }}$} & $240.15 \mathrm{~K}$ & & \\
\hline & $k_{f}$ & $c_{f}$ & $\mu_{f}$ \\
\hline$a_{0}$ & 0.198722 & 3460.27 & 57474.9042 \\
\hline$a_{1}$ & 0.151743 & -439.50 & -332716.4882 \\
\hline$a_{2}$ & -0.016103 & 436.08 & 824928.7105 \\
\hline$a_{3}$ & & & -1135480.0882 \\
\hline$a_{4}$ & & & 937049.2192 \\
\hline$a_{5}$ & & & -463600.2822 \\
\hline$a_{6}$ & & & 127315.5228 \\
\hline$a_{7}$ & & & -14971.1625 \\
\hline
\end{tabular}

Where $T_{\text {solid }}$ is the freezing point of the fluid. Figure 2.8 shows a curve fit of $k_{f}$ as a function of the reduced temperature for water. 


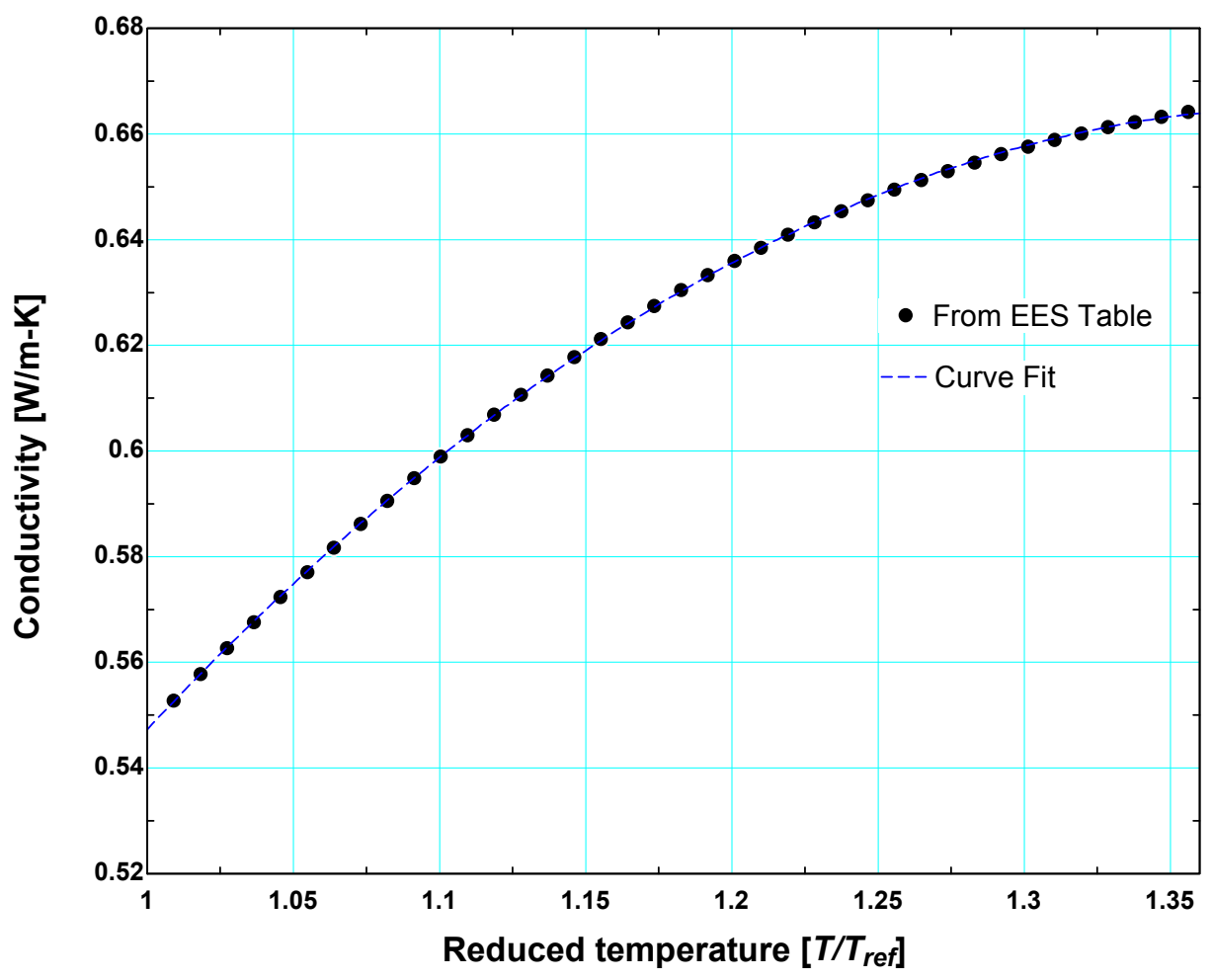

Figure 2.8. Actual and Curve Fit Data for $k_{f}$ vs. $T / T_{r e f}$

The enthalpy for each fluid is determined by integrating the $c_{f}(T)$ function with respect to temperature. The fluid is assumed to be incompressible and the integral is shown in Eq. (2.33). The zero reference enthalpy is chosen at $T_{r e f}$, where $T_{r e f}$ may be different for each fluid.

$$
h_{f}(T)=\int_{T_{r e f}}^{T} c_{f}(T) d T
$$

Figure 2.9 illustrates $c_{f}(T)$ for the different fluids considered for the AMRR application. 


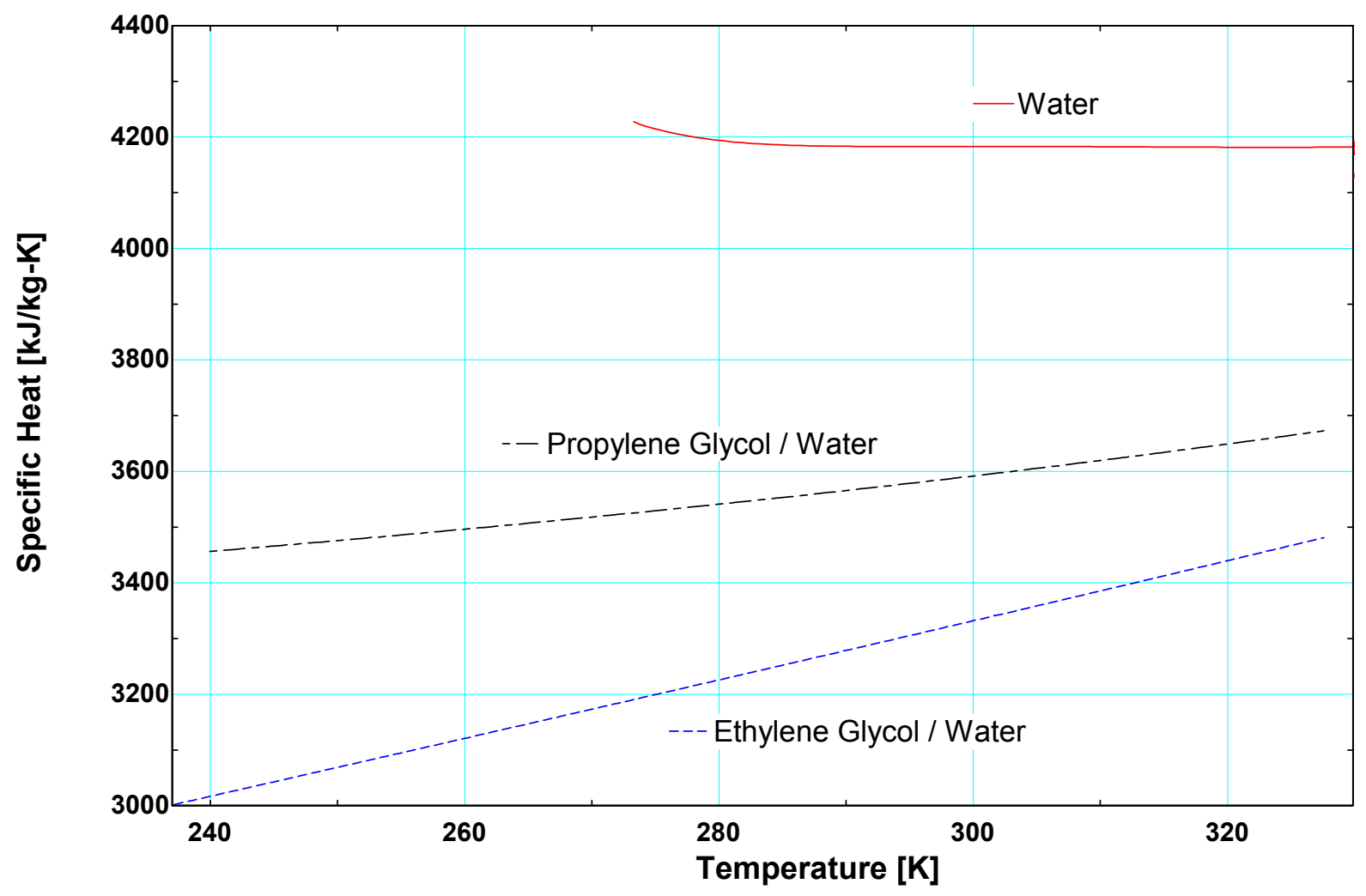

Figure 2.9. Specific heat as a function of temperature for some possible working fluids

\subsubsection{Magnetocaloric Material Properties}

Experimental property data for a 94\% gadolinium $6 \%$ erbium alloy, $\mathrm{Gd}_{0.94} \mathrm{Er}_{0.06}$, measured at Ames Lab and provided by Astronautics Corporation were used to provide entropy over a range of temperatures and applied fields, and the data is shown in Figure 2.10 . 


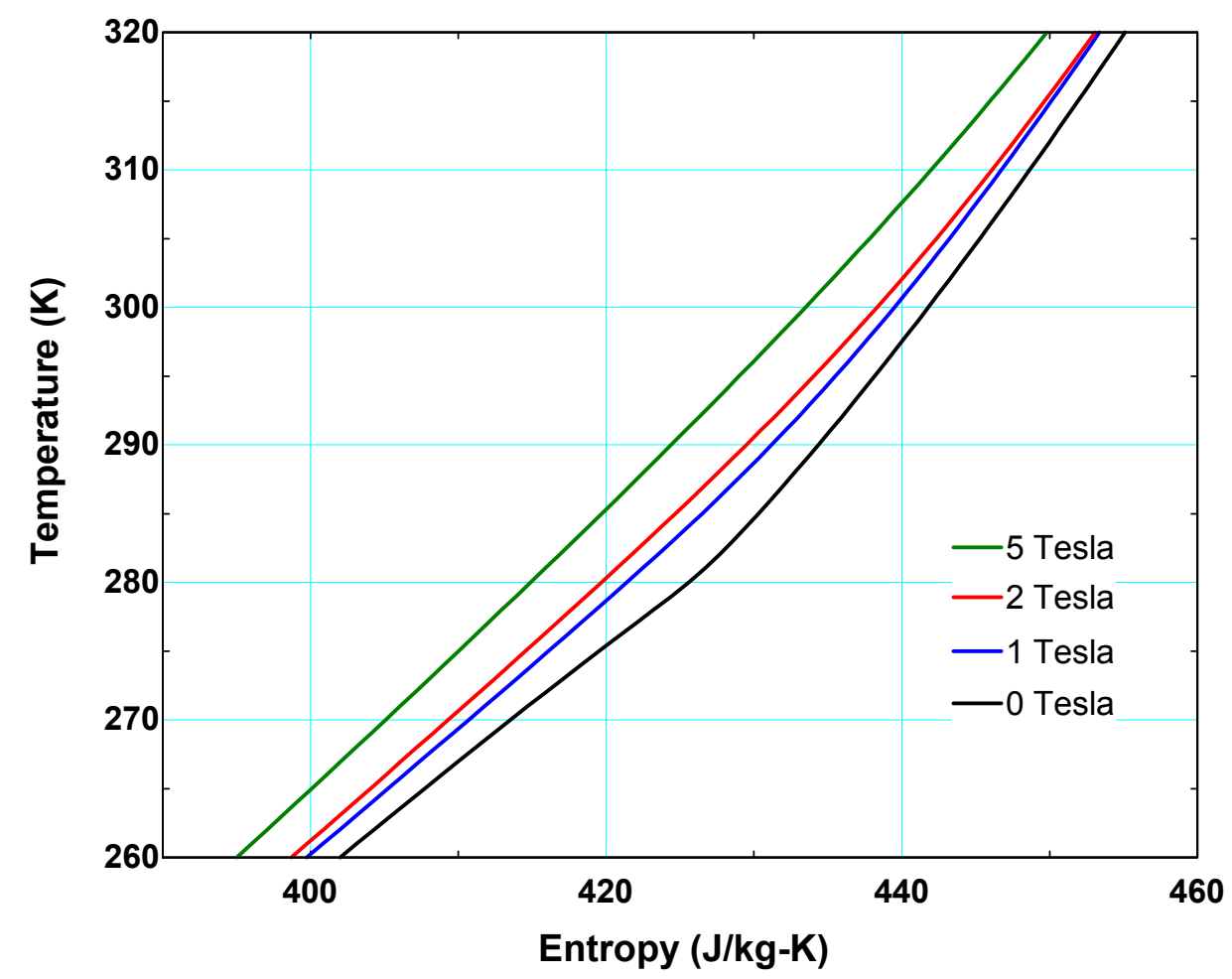

Figure 2.10. Temperature-entropy diagram for $\mathrm{Gd}_{0.94} \mathrm{Er}_{0.06}$

These experimental data are interpolated using a two-dimensional spline technique and the interpolated entropy data are numerically differentiated in order to determine the required model inputs: constant field specific heat capacity and the partial derivative of entropy with respect to applied field. To determine material properties when the Curie point of the material is shifted, the shape of the material properties is assumed constant and is shifted along the temperature scale according to the specified Curie point. Shifting the Curie point in the model is accomplished by evaluating the properties of the $94 \%$ gadolinium 6\% erbium alloy at temperatures that are modified according to Eq. (2.34). 


$$
c_{\mu_{o} H}\left(T, T_{\text {curie }}, \mu_{o} H\right)=c_{\mu_{o} H, \text { ref }}\left(T \frac{T_{\text {Curie }}}{T_{\text {Curie, }, \text { ref }}}, T_{\text {curie }}, \mu_{o} H\right)
$$

where $T_{\text {Curie,ref }}$ is the Curie temperature of the experimental property data and $c_{\mu_{o} H, \text { ref }}$ is the constant magnetic field specific heat of the experimental data. Property data for $\frac{\partial s}{\partial \mu_{o} H}$ are obtained in the same manner. Based on material property data in Chen et al. (2003), material properties of tunable alloys can exhibit nearly identical property shapes that are shifted along the temperature axis according to the Curie temperature of the material. Therefore, shifting material properties based on the Curie temperature of the alloy can provide reasonable material properties in a layered bed. By shifting the experimental properties along the temperature scale as shown in Eq. (2.34), material properties can be estimated for alloys with any desired Curie temperature without requiring detailed experimental data for each material.

A magnetic equation of state was initially developed and used to obtain the magnetic properties required to implement the numerical model. It was difficult to obtain a functional form for the magnetic equation of state that fulfilled all of the thermodynamic requirements (e.g., continuity of $1^{\text {st }}$ derivative, integratability, etc.) and still accurately represented the data obtained from Astronautics Inc. Small deviations from the data, particularly where the magnetocaloric effect is weak, can result in large changes in performance; this is especially true when modeling a non-layered bed where the bed must operate in these non-optimal thermodynamic regions. The best-fit approximation of the 
magnetic material properties is shown in Figure 2.11, note that agreement between experimental data and the curve fit was poor.

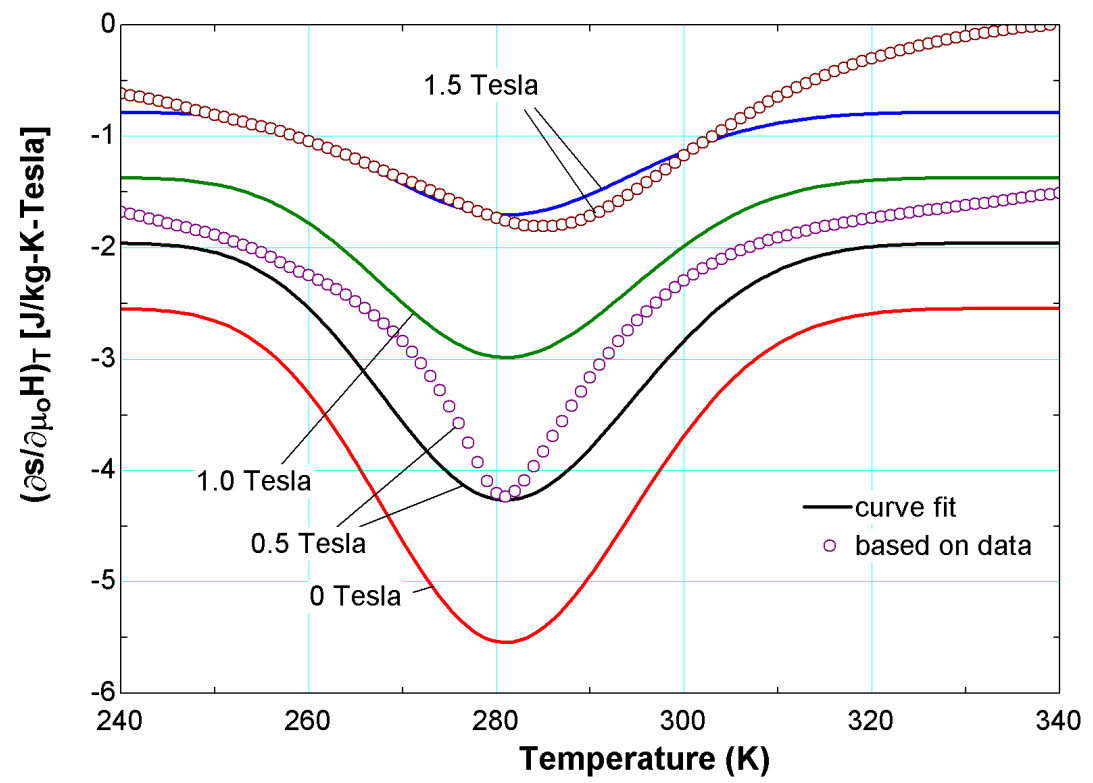

Figure 2.11. Partial derivative of entropy with respect to applied field based on data and from analytical function.

The benefit of the magnetic equation of state approach was computational efficiency and absolutely thermodynamically consistent properties. The drawback is the lack of accuracy and flexibility with regards to simulating various materials. Therefore, at the suggestion of Astronautics, bi-cubic spline interpolation of entropy data spaced over uniform grid of temperature and applied field has been implemented in order to obtain the property data. This approach is less computationally efficient and, due to limitations in the interpolation technique, not completely thermodynamically consistent. In other words, the data itself may not be completely thermodynamically consistent due to experimental errors. An entropy surface (in the temperature-applied field space) cannot have an arbitrary shape; it must satisfy some constraints associated with thermodynamics. 
For example, entropy must always increase with temperature. Any inconsistencies in the data will be reflected in the entropy surface obtained by an interpolation procedure. However, we have found that proper implementation of the interpolation results in minimal thermodynamic inconsistency (as evident by the local cyclic balance between fluid and matrix entropy changes) and a computationally efficiency model.

\subsection{Regenerator Correlations}

\subsubsection{Pressure Drop}

The Ergun (1952) equation is used to predict pressure drop in a packed particle porous media. The equation calculates the negative of pressure gradient as shown in Eq. (2.35).

$$
\begin{aligned}
& \frac{d P}{d x}=\left(\frac{A \alpha \mu}{d_{h}^{2}}\right) v+\left(\frac{B \beta \rho}{d_{h}}\right) v^{2} \\
& \alpha=\frac{(1-\varepsilon)^{2}}{\varepsilon^{2}} \text { and } \beta=\frac{1-\varepsilon}{\varepsilon^{3}}
\end{aligned}
$$

where $d_{h}$ is the diameter of the regenerator packing material and $v$ is the open area fluid velocity. Kaviany (1995) suggests a value of 180 for the constant $A$ and 1.8 for $B$ for smooth particles. The pressure gradient from Equation (2.35) is converted to a friction factor for use in the governing equations and rearranged in terms of Reynolds number.

$$
f_{f}=360 \frac{(1-\varepsilon)^{2}}{\varepsilon^{3} \cdot R e_{f}}+3.6 \frac{1-\varepsilon}{\varepsilon^{3}}
$$

From Rohsenow (1998), the friction factor of a packed bed of spheres is

$$
f=300 \frac{(1-\varepsilon)^{2}}{\varepsilon^{3} \cdot R e_{f}}+3.75 \frac{1-\varepsilon}{\varepsilon^{3}}
$$


Based on experimental findings by Astronautics for packed sphere regenerators, Equation (2.36) suggested by Kaviany predicts the pressure drop within acceptable accuracy.

\subsubsection{Heat Transfer}

Wakao and Kaguei (1982) have fit data for air and other gases flowing through a range of matrix geometries (cylinders, spheres, sintered spheroids) made from a number of materials (Celite, steel, bronze, glass, alumina) and suggest the following empirical correlation for Nusselt number in a packed sphere bed

$$
N u_{f}=2+1.1 \operatorname{Re}_{f}^{0.6} \operatorname{Pr}_{f}^{1 / 3}
$$

This correlation is applicable for all values of Reynolds numbers. Rohsenow also presents a Nusselt number correlation for packed spheres

$$
N u_{f}=2+1.8 \operatorname{Re}_{f}^{1 / 2} \operatorname{Pr}_{f}^{1 / 3}
$$

for $R e_{f}>50$. Because the Reynolds number in an AMRR regenerator may be below 50 in practical applications, the correlation presented by Wakao and Kaguei was used. A comparison of the two correlations is shown in Figure 2.12. 


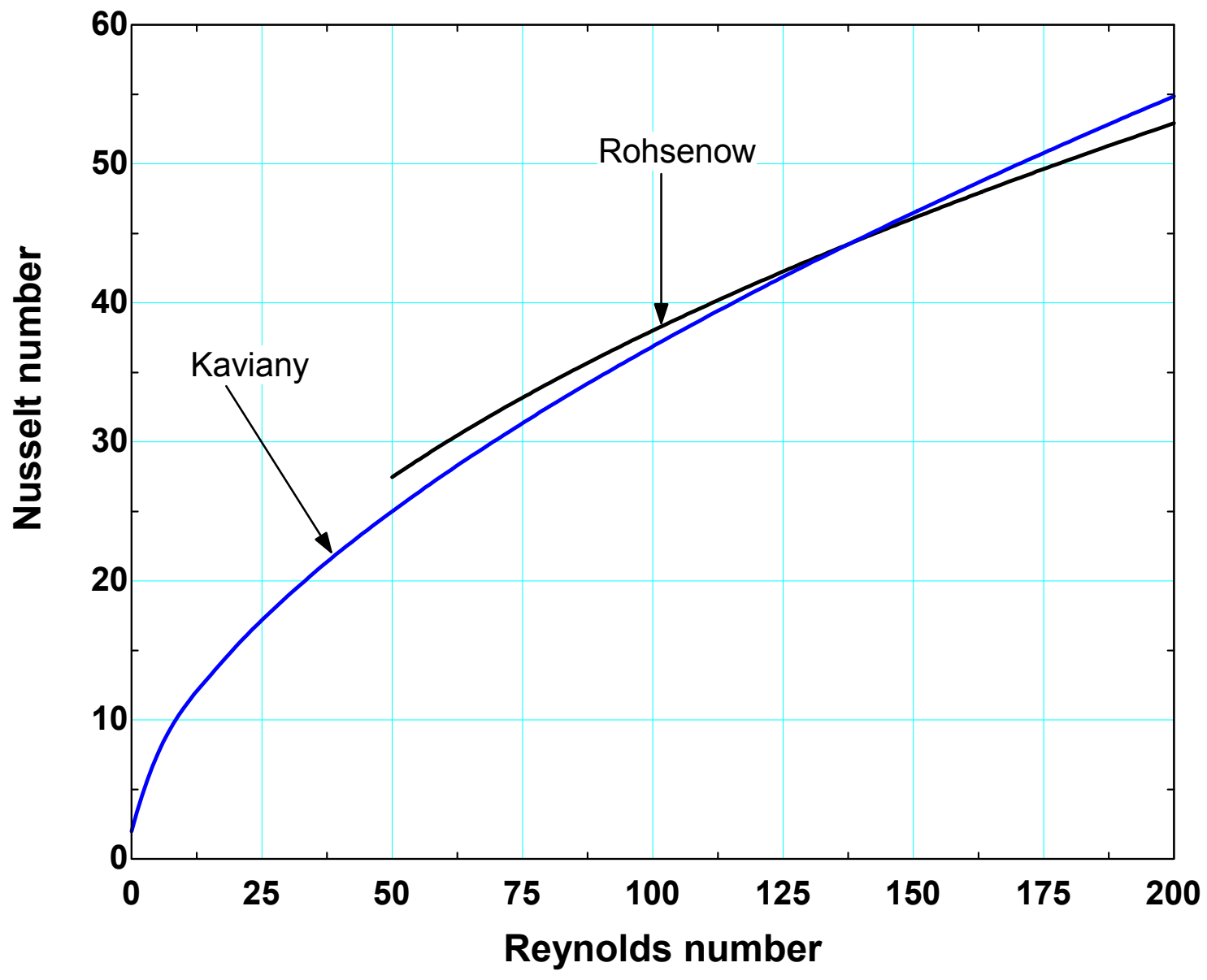

Figure 2.12. Comparison of Nusselt number correlations

\subsubsection{Axial Conduction}

Dispersion in the regenerator acts to mix fluid along the bed in the direction of flow and can be treated as an axial conduction term. Therefore, the total axial conductivity of a regenerator bed is a function of dispersion, $k_{f} D^{d}$, and static effective thermal conductivity, $k_{\text {static. }} D^{d}$ is a unitless dispersion coefficient that is a function of the Peclet number of the fluid. From Kaviany (1995), the total effective conductivity, $k_{\text {eff, }}$ can be expressed as

$$
k_{\text {eff }}=k_{\text {static }}+k_{f} D^{d}
$$


where $k_{\text {static }}$ is the effective conductivity of the regenerator bed when there is no flow. Kunii and Smith (1960) developed a method to determine the effective static thermal conductivity of a matrix of packed spheres in a fluid using equation (2.41). In this equation, $\phi$ is a function of porosity, and $\beta$ is an experimentally determined function of the bed packing geometry that ranges from $0.9-1$. Because the value of $\beta$ does not greatly affect values of $k_{\text {static }}$ and is generally very close to $1, \beta$ is initially set to 1 for this study.

$$
k_{\text {static }}=k_{f}\left(\varepsilon+\frac{\beta(1-\varepsilon)}{\phi+\frac{k_{f}}{k_{r}}}\right)
$$

for $\varepsilon<0.476$. Hadley (1986) presents a correlation for the static fluid conductivity in a periodic porous structure

$$
\begin{aligned}
& k_{\text {static }}=k_{f}\left[\left(1-\alpha_{0}\right) \frac{\varepsilon f_{0}+k_{r} / k_{f}\left(1-\varepsilon f_{0}\right)}{1-\varepsilon\left(1-f_{0}\right)+k_{r} / k_{f} \varepsilon\left(1-f_{0}\right)}+\alpha_{0} \frac{2\left(k_{r} / k_{f}\right)^{2}(1-\varepsilon)+(1+2 \varepsilon) k_{r} / k_{f}}{(2+\varepsilon) k_{r} / k_{f}+1-\varepsilon}\right] \\
& f_{0}=0.8+0.1 \varepsilon \\
& \log \alpha_{0}=-4.898 \varepsilon, \quad 0 \leq \varepsilon \leq 0.0827 \\
& \log \alpha_{0}=-0.405-3.154(\varepsilon-0.0827), \quad 0.0827 \leq \varepsilon \leq 0.298 \\
& \log \alpha_{0}=-1.084-6.778(\varepsilon-0.298), \quad 0.298 \leq \varepsilon \leq 0.580
\end{aligned}
$$

for $\varepsilon<0.58$. Kaviany suggests a correlation to determine the effective conductivity that was developed by Krupiczka in 1967 and is shown in equation (2.45). 


$$
k_{\text {static }}=k_{f}\left(\frac{k_{r}}{k_{f}}\right)^{0.28-0.757 \log \varepsilon-0.057 \log \left(k_{r} / k_{f}\right)}
$$

The predictions of these two correlations for effective static conductivity were compared for the typical conditions seen in the regenerator bed. The fluid is water $\left(k_{f}=0.6 \mathrm{~W} / \mathrm{m}\right.$ $\mathrm{K})$ and Gadolinium is used as the matrix material $\left(k_{r}=10 \mathrm{~W} / \mathrm{m}-\mathrm{K}\right)$ over a range of porosities. The results are shown in Figure 2.13. The correlation proposed by Hadley is used in the numerical model because the work is more recent and uses a more detailed approach.

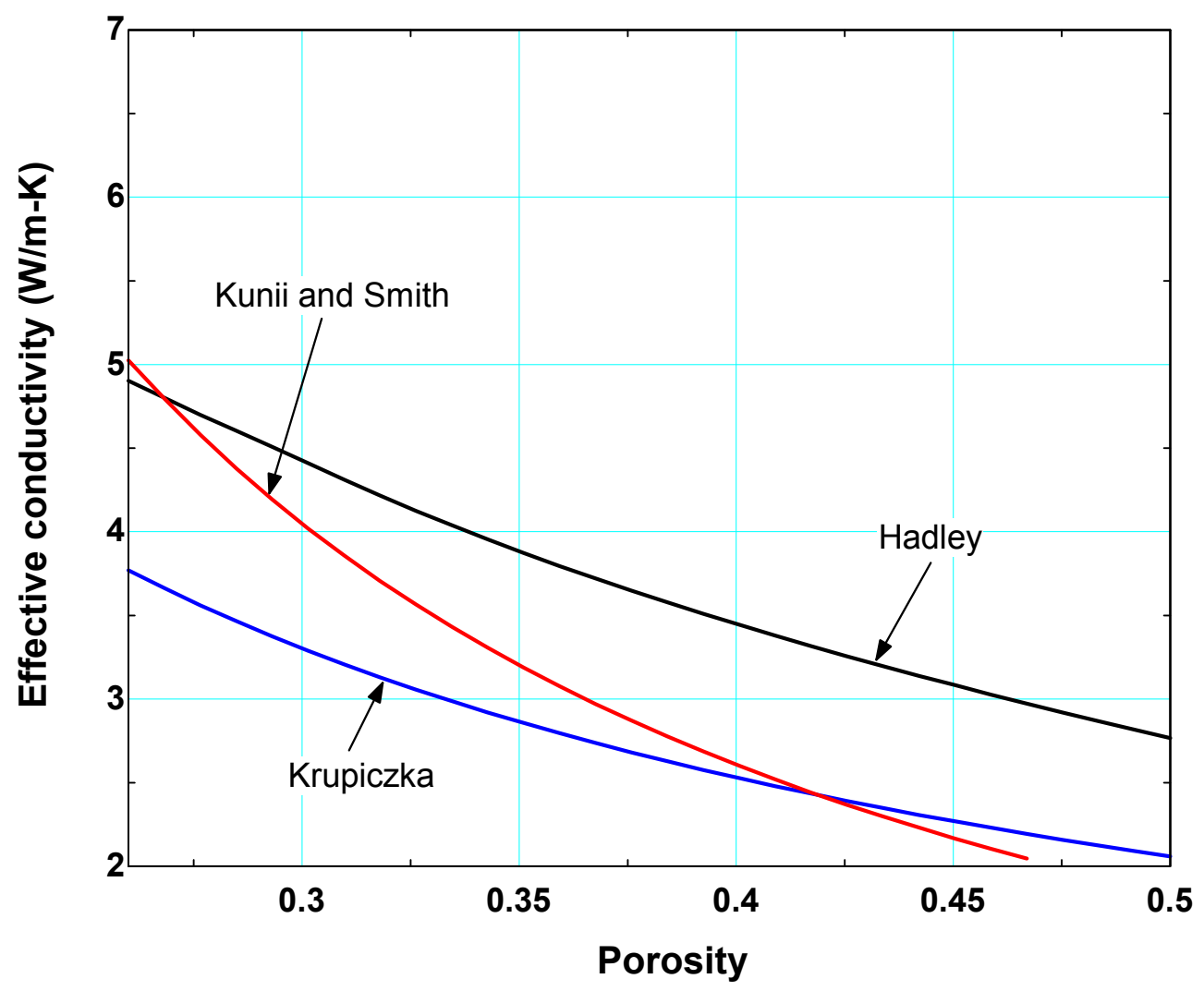

Figure 2.13. Comparison of effective static conductivity models under typical AMRR operating conditions 
A theoretical model of dispersion in a uniformly packed bed of spheres is presented by Kaviany and is given in Equation (2.46).

$$
D^{d}=\varepsilon \frac{3}{4} P e_{f}, \quad P e_{f} \gg 1
$$

where $P e$ is the Peclet number of the flow, defined as $P e=R e_{f} P r_{f}$. Vafai (2000) presents an empirical correlation for thermal dispersion in the direction of flow shown in equation (2.47).

$$
D^{d}=0.5 \mathrm{P} e_{f}
$$

A correlation developed by Kamiuto and Saitoh in 1996 is also presented by Vafai.

$$
D^{d}=0.3519(1-\varepsilon)^{2.3819} P e_{f}
$$

Yagi and Wakao from 1959 suggest the following value for dispersion in a constant porosity packed tube according to Vafai.

$$
D^{d}=0.1 P e_{f}
$$

Equation (2.46) is used in the current model because it is a recent correlation and its predictions are between the maximum and minimum predictions presented here. The values for static conduction and dispersion coefficient, $D^{d}$, may be substituted into Equation (2.40) in order to determine the total effective conductivity of the regenerator bed with axial dispersion. 


\subsection{Correction for Internal Temperature Gradients}

The Biot number is tradionally used to determine whether the thermal resistance with a solid material can be ignored. The Biot number, $B i$, is defined in terms of the heat

transfer coefficient between the fluid and the regenerator, $h$, as $B i=\frac{h \cdot d_{h}}{2 k_{r}}$. Values of

Biot number less than 0.1 indicate that the resistance within a solid is small relative to resistance at the solid surface. The Biot number associated with the magnetic material is not generally less than 0.1 throughout the entire cycle. Therefore, the temperature gradients that exist within the regenerator matrix are not negligible relative to the temperature difference between the matrix and the fluid. As a result, there is a significant entropy generation related to conduction from the center of the matrix to the outer surface as well as from convection from the matrix surface to the fluid. This effect is approximately accounted for by determining the difference between the volumetric mean temperature of the sphere and its surface temperature and using this result to modify the heat transfer coefficient. This method has been used previously for rock beds by Jeffreson (1972) .

In the case of a matrix composed of spheres, each sphere is modeled as having a uniform internal heat generation per unit volume, $\dot{g}^{\prime \prime \prime}$. The governing equation for this situation is:

$$
4 \pi r^{2} \dot{g}^{\prime \prime \prime}=\frac{d}{d r}\left(-k_{r} 4 \pi r^{2} \frac{d T}{d r}\right)
$$


Rearranging terms yields:

$$
d\left[r^{2} \frac{d T}{d r}\right]=-\frac{r^{2} \dot{g}^{\prime \prime}}{k_{r}} d r
$$

Integrating twice leads to:

$$
T=-\frac{r^{2} \dot{g}^{\prime \prime}}{6 k_{r}}-C_{1} r^{-1}+C_{2}
$$

The boundary conditions are:

$$
\begin{aligned}
& \left.\frac{d T}{d r}\right|_{r=0}=0 \\
& T\left(r=r_{o}\right)=T_{s}
\end{aligned}
$$

where $r_{o}$ is the radius of the sphere and $T_{s}$ is the surface temperature. $C_{l}$ must be zero in order to satisfy the first boundary condition as well as to avoid a singularity at $r=0$. The second boundary condition allows $C_{2}$ to be solved in terms of $T_{s}$ in order to obtain the temperature profile through the sphere.

$$
T(r)=T_{s}+\frac{r_{o}^{2} \dot{g}^{\prime \prime \prime}}{6 k_{r}}-\frac{r^{2} \dot{g}^{\prime \prime}}{6 k_{r}}
$$

The volumetric average temperature of the sphere material can be determined by integrating the temperature distribution.

$$
\bar{T}=\frac{3}{4 \pi r_{o}^{3}} \int_{0}^{r_{o}}\left[T_{s}+\frac{r_{o}^{2} \dot{g}^{\prime \prime \prime}}{6 k_{r}}-\frac{r^{2} \dot{g}^{\prime \prime \prime}}{6 k_{r}}\right] d r
$$

Carrying out the integration, the average temperature is: 


$$
\bar{T}=\frac{1}{15} \frac{\dot{g}^{\prime \prime} r_{o}^{2}}{k_{r}}+T_{s}
$$

Equation (2.56) can be written in terms of the heat transfer from the sphere, $\dot{q}$ where $\dot{q}=4 / 3 \pi r_{o}^{3} \dot{g}^{\prime \prime}$

$$
\bar{T}=T_{s}+\frac{\dot{q}}{20 \pi r_{o} k_{r}}
$$

The temperature of the surface of the sphere can be related to the temperature of the fluid to which it is convecting according to:

$$
T_{s}=T_{f}+\frac{\dot{q}}{h 4 \pi r_{o}^{2}}
$$

where $h$ is the heat transfer coefficient. Substituting (2.57) into (2.58) and solving for $\bar{T}$ yields:

$$
\bar{T}=T_{f}+\frac{\dot{q}}{h 4 \pi r_{o}^{2}}+\frac{\dot{q}}{20 \pi r_{o} k_{r}}
$$

which simplifies to:

$$
\bar{T}-T_{f}=\frac{\dot{q}}{h 4 \pi r_{o}^{2}}\left(1+\frac{r_{o} h}{5 k_{r}}\right)
$$

Substituting the definition of the Biot number $\left(B i=r h / k_{r}\right)$ into equation (2.60) yields

$$
\bar{T}-T_{f}=\frac{\dot{q}}{h 4 \pi r_{o}^{2}}\left(1+\frac{B i}{5}\right)
$$


which suggests the definition of a modified or effective heat transfer coefficient, $h_{\text {eff }}$, to correct for temperature gradients in the regenerator spheres:

$$
h_{e f f}=\frac{h}{1+\frac{B i}{5}}
$$

Although the derivation above was carried out for spheres, the technique is equally valid for alternative matrix geometries provided that the Biot number is appropriately defined.

\subsection{Correction for Entrained Fluid Heat Capacity}

Regenerative heat exchangers are used in many applications including cryogenic refrigeration systems, building energy recovery, and gas turbines. The heat capacity of the fluid, often a gas that is entrained in the void volume of the regenerator matrix, is typically very small relative to the heat capacity associated with the regenerator matrix itself. However, AMRR systems use heat transfer fluids such as water, and the heat capacity of the fluid entrained in the matrix is non-negligible in this application as the capacity of the entrained fluid may be about equal to the matrix heat capacity in a practical design. As a result, the entrained fluid heat capacity should not be neglected when modeling an AMRR system. Nellis and Klein (2004) developed a method to correct for the lumped capacitance assumption used to derive the regenerator model; that is, the combining of the fluid and regenerator heat capacity in the governing equations. The method suggests enhancing the heat transfer between the fluid and regenerator in order to correct for the lumped capacitance assumption. In the regenerator model, the fluid to regenerator heat transfer is calculated using an augmented heat transfer 
coefficient, $h_{\text {aug }}$, in order to correct for lumped capacitance. The augmented number of transfer units is given by

$$
h^{*}=h_{\text {aug }}\left(1+1.7640 R+1.0064 R^{2}\right)
$$

where $R$ is the ratio of fluid heat capacity to the regenerator heat capacity defined in equation (2.64). For an AMRR with water as the heat transfer and a GdEr alloy as the solid refrigerant, $R$ is typically near 1 .

$$
R=\frac{\rho_{f} c_{f} \varepsilon}{\rho_{r} c_{r}(1-\varepsilon)}
$$

This method of correcting for the lumped capacitance assumption has been verified experimentally on a passive regenerator experimental setup. The results are presented in Chapter 3.

\subsection{Validation of the Numerical Model}

Initial checks of the model described in section 2.3 used constant property fluids and magnetic materials as well as a spatially uniform magnetic field over the regenerator bed. As the model becomes increasingly complex with regard to material properties, magnetic field variation, regenerator geometry, and other cycle considerations, it is important to continually check that the model is predicting results that are numerically stable and thermodynamically consistent. With this in mind, the following checks on the model predictions have been developed and were used whenever a new element was added to the model. 
Figure 2.14 shows a schematic of energy flow in the regenerator. Energy enters the regenerator as heat transfer from the cold reservoir and magnetic work interaction with the magnetic regenerator material. The magnetic work is converted to heat transfer through a thermal resistance. The incoming energy is carried through the bed from cold to hot as a net enthalpy flux. The net enthalpy flux at the hot end of the regenerator becomes the heat rejected to the hot reservoir. A differential segment of the regenerator in Figure 2.14 is shown in Figure 2.15.

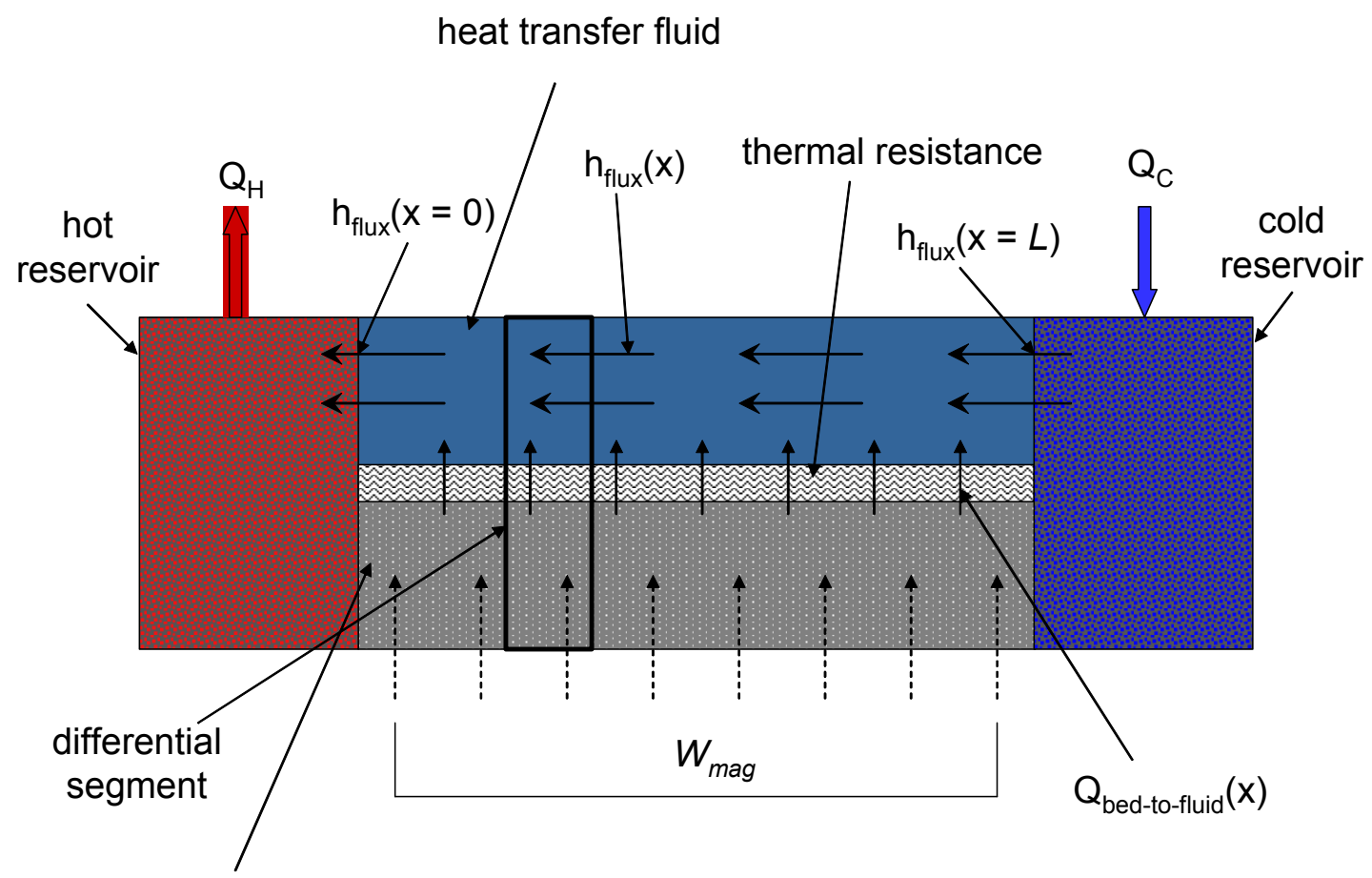

Magnetic regenerator material

Figure 2.14. Schematic of energy flow in the regenerator 


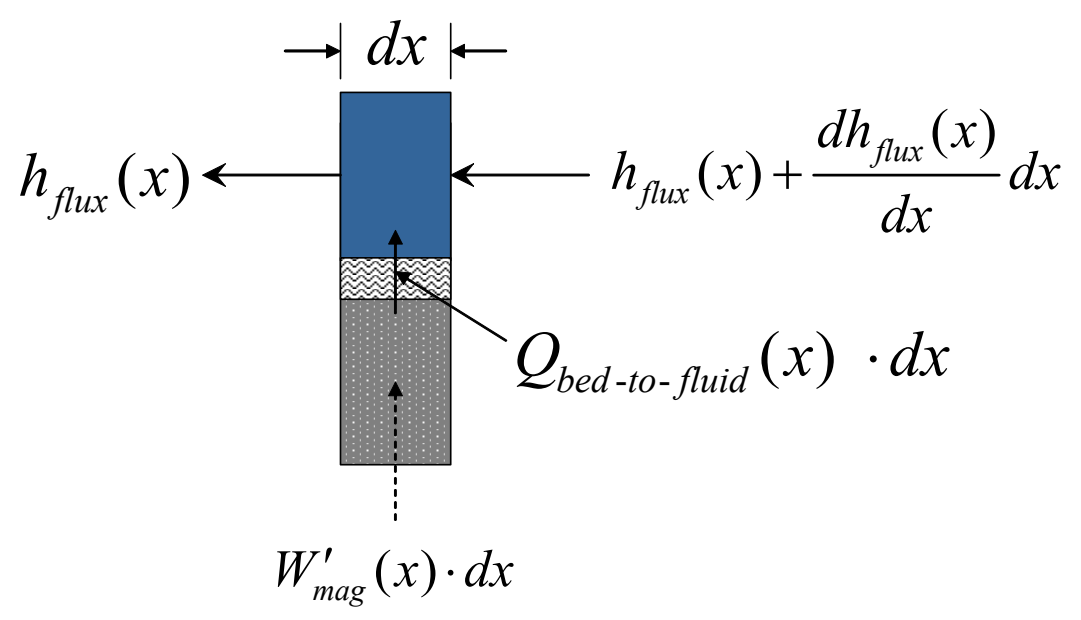

Figure 2.15. Differential segment for energy flow

Figure 2.16 shows a schematic of entropy flow in the regenerator. Similar to enthalpy flow, entropy enters the regenerator as heat transfer from the cold reservoir. There is no entropy interaction due to magnetic work because it is modeled as a reversible process. The entropy from the cold reservoir is carried through the bed from the cold to hot end as a net entropy flux. Entropy is also generated along the bed due to heat transfer $\left(\dot{S}_{g e n, Q}\right)$ through the thermal resistance separating the magnetic material from the fluid. The net entropy flux at the hot end of the regenerator is rejected in the form of heat transfer to the hot reservoir. A differential segment of the regenerator in Figure 2.16 is shown in Figure 2.17 . 


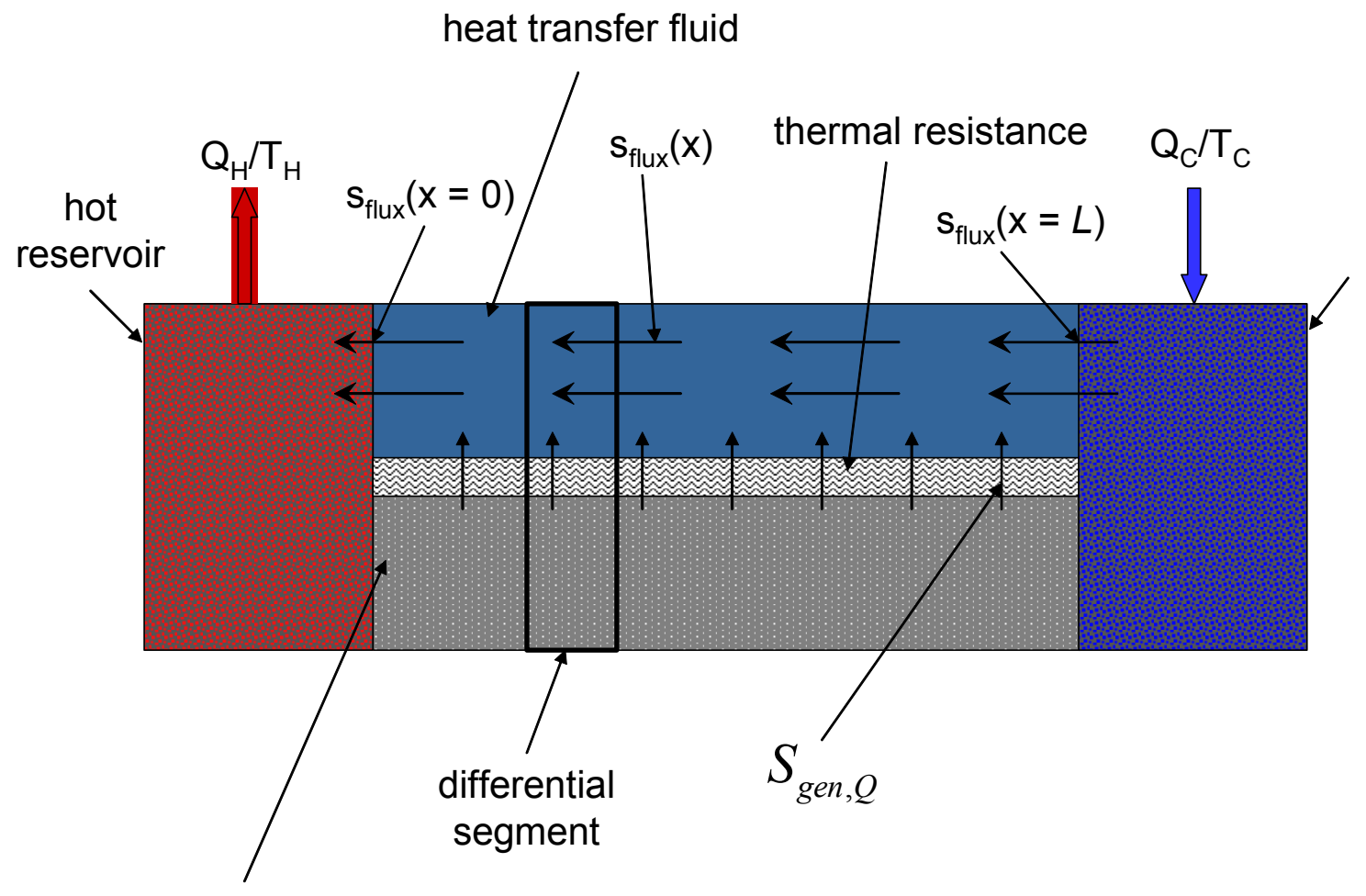

Magnetic regenerator material

Figure 2.16. Schematic of entropy flow in the regenerator

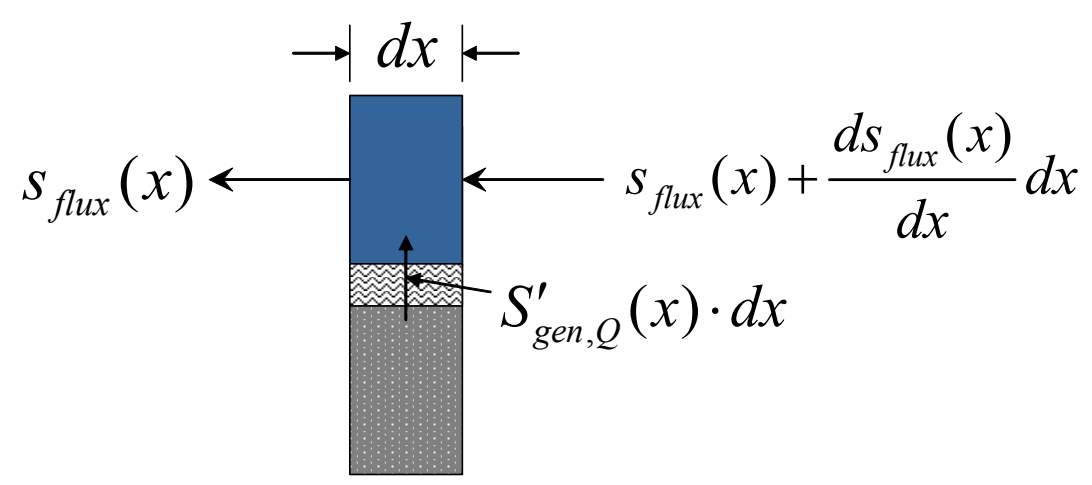

Figure 2.17. Differential segment for entropy flow 
The basic outputs of the model are the refrigeration capacity, heat rejected, and the magnetic work into the regenerator. In this case, the work input to the bed from the magnet is related to the work provided by a motor in order to rotate the regenerator through the magnet field. However, the magnetic work does not include any irreversibilities associated with hysteresis, motor inefficiency, bearing friction, etc; note that these losses are separately calculated in the system model. In this limit, the net amount of heat transfer to the regenerator material per unit length of bed at any location, $Q^{\prime}(x)$, can be calculated by integrating the temperature with respect to entropy for each segment of the bed.

$$
Q^{\prime}(x)=\rho_{r} A_{c}(1-\varepsilon) \int_{0}^{\tau} T_{r}(x, t) \frac{d s_{r}(x, t)}{d t} d t
$$

where $T_{r}$ and $s_{r}$ are the temperature and entropy of the magnetic material predicted by the model as a function of space and time, $\rho_{r}$ is the density of the material, $A_{c}$ is the crosssectional area of the bed, and $\varepsilon$ is the porosity of the bed. The governing equations in the model are derived by assuming that the magnetic material undergoes internally reversible processes. Therefore, the work input per unit length of the bed over a steady-state cycle $\left(W_{m a g}^{\prime}\right)$ must be equal to the heat input per unit length of bed calculated according to Equation (2.66).

$$
W_{\text {mag }}^{\prime}(x)=Q^{\prime}(x)
$$

The integration in Equation (2.66) is performed numerically in the model using the trapezoid method. The work done by the magnet over one cycle is calculated by 
summing values for work input per unit length from equation (2.66) and multiplying by the length of the regenerator as shown in (2.67).

$$
\oint T d s=W_{\text {mag }}=L \cdot \sum^{L} Q^{\prime}(x)
$$

The magnet work results in a net heat transfer to the regenerator which is transferred to the working fluid in the form of increase in enthalpy flux. The work of the magnet can therefore be determined independently by taking the difference in the enthalpy flux at the cold and hot reservoirs. The net enthalpy flux through each spatial step in the regenerator bed is calculated in the model. Figure 2.18 shows a plot of net enthalpy flux across a regenerator of length $0.25 \mathrm{~m}$ during a steady-state cycle. The difference between the net enthalpy flux at the hot end $\left(x_{r}=0\right)$ and the cold end $\left(x_{r}=L\right)$ is the net energy transported away from the regenerator by the fluid. For steady state operation of the system, the work transferred to the regenerator by the magnet must equal the net energy transferred from the regenerator to the fluid. If the two values differ, the solution is not thermodynamically consistent. 


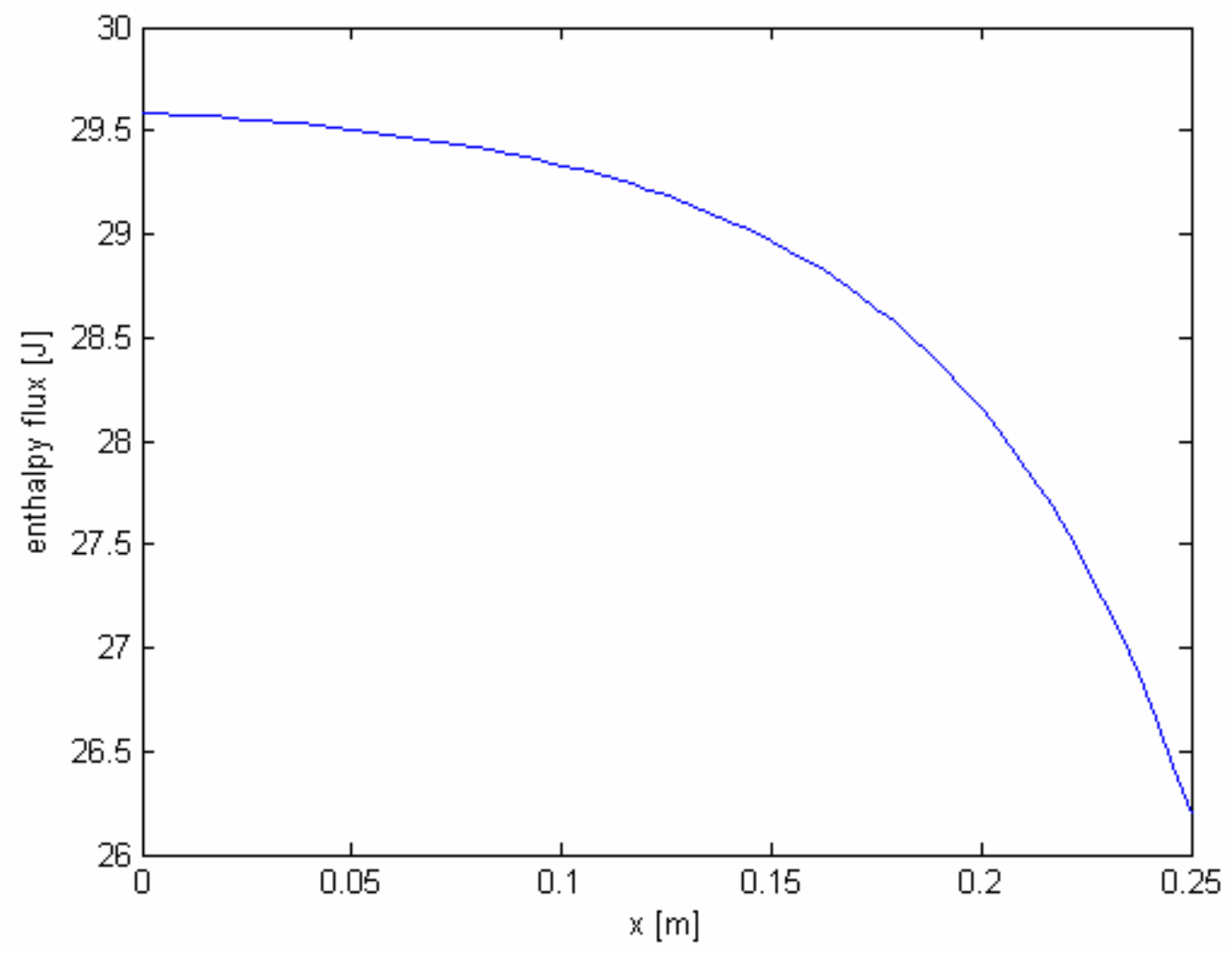

Figure 2.18. Enthalpy flux plot for a steady-state cycle

The heat rejection and refrigeration loads for the cycle can be determined by calculating the net heat flux carried by the fluid through the hot and cold end of the regenerator; these values are calculated numerically in the model as shown below. In Eq. (2.68) $h_{f}[\mathrm{i}, 1]$ is the enthalpy at the first spatial step in the regenerator bed $\left(x_{f}=0\right)$ which is nearest the hot reservoir. Similarly, the $n^{\text {th }}$ spatial step in Eq. (2.69) is nearest the cold reservoir.

$$
Q_{\text {rejected }}=h_{f l u x, H}=\sum_{i=1}^{m} \dot{m}_{f}[i] h_{f}[i, 1] \frac{\tau}{m}
$$




$$
Q_{\text {refrigeration }}=h_{f l u x, C}=\sum_{i=1}^{m} \dot{m}_{f}[i] h_{f}[i, n] \frac{\tau}{m}
$$

By performing a first law energy balance, the work input of the magnet is given as the difference between the heat rejected and the refrigeration load.

$$
W_{\text {mag }}=Q_{\text {rejected }}-Q_{\text {refrigeration }}
$$

The results of the model are checked by verifying that $W_{\text {mag }}$ calculated independently using (2.67) and (2.70) are equal to within numerical accuracy. If the two values are not equal, then the model is not thermodynamically consistent.

Entropy production, $\dot{S}_{g e n}$, for any cycle must be greater than or, in the reversible limit, equal to zero. To check that the entropy production is positive for the model, the entropy flux through each spatial step in the regenerator is calculated in the same manner as the enthalpy flux and is shown in Eq. (2.71). Figure 2.18 shows a graph of entropy flux for a bed of length $0.25 \mathrm{~m}$. The fluid entering the bed from the cold reservoir increases in entropy as it increases in temperature and is rejected to the hot reservoir. This process is shown in Figure 2.18 where the entropy rejection (negative entropy flux) at the hot reservoir is greater than at the cold reservoir. This shows that entropy production for the cycle is positive. The entropy rejected in the figure is decreasing from the hot end to the cold end of the bed which also shows that entropy production across the cycle is positive.

$$
s_{f l u x}[j]=\sum_{i=1}^{m} \dot{m}_{f}[i] s_{f}[i, j] \frac{\tau}{m}
$$




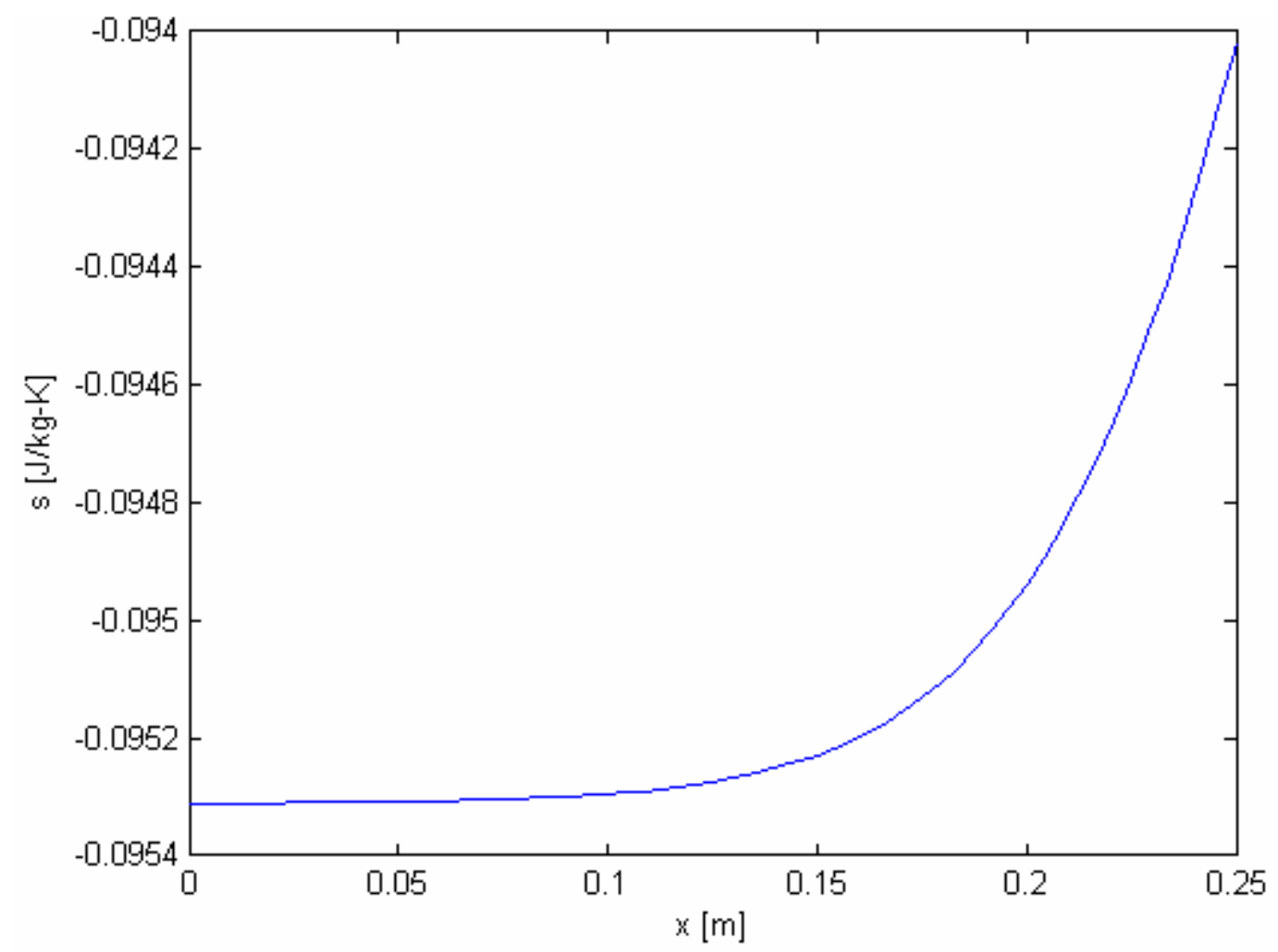

Figure 2.18. Entropy Flux in the Regenerator

A final investigation of the cycle can be realized by examining a temperature-entropy plot for the magnetic material over a steady-state cycle at selected axial locations within the regenerator. The $T-s$ diagram of a consistent model for a steady-state cycle is closed, as shown in Figure 2.19. Qualitatively, it should have a shape that makes sense for the cycle and also helps with the visualization of the cycle. Temperature-entropy diagrams can be plotted for any desired segment of the regenerator; an example of a $T-s$ diagram at an arbitrary location in the regenerator is shown in Figure 2.19. In Figure 2.19, the process from state $1-2$ is magnetization; note that the magnetization and demagnetization of the regenerator result in a change in entropy. Although the 
magnetization/demagnetization of the magnetic material is considered reversible, there is a decrease in entropy from state $1-2$ related to the fact that the magnetic material transfers heat to the cooler fluid through a finite temperature difference as it increases in temperature due to the increased magnetic field (the magnetocaloric effect). $2-3$ is cold-to-hot flow which is heat rejection. $3-4$ is demagnetization. $4-1$ is hot-to-cold flow which results in refrigeration.

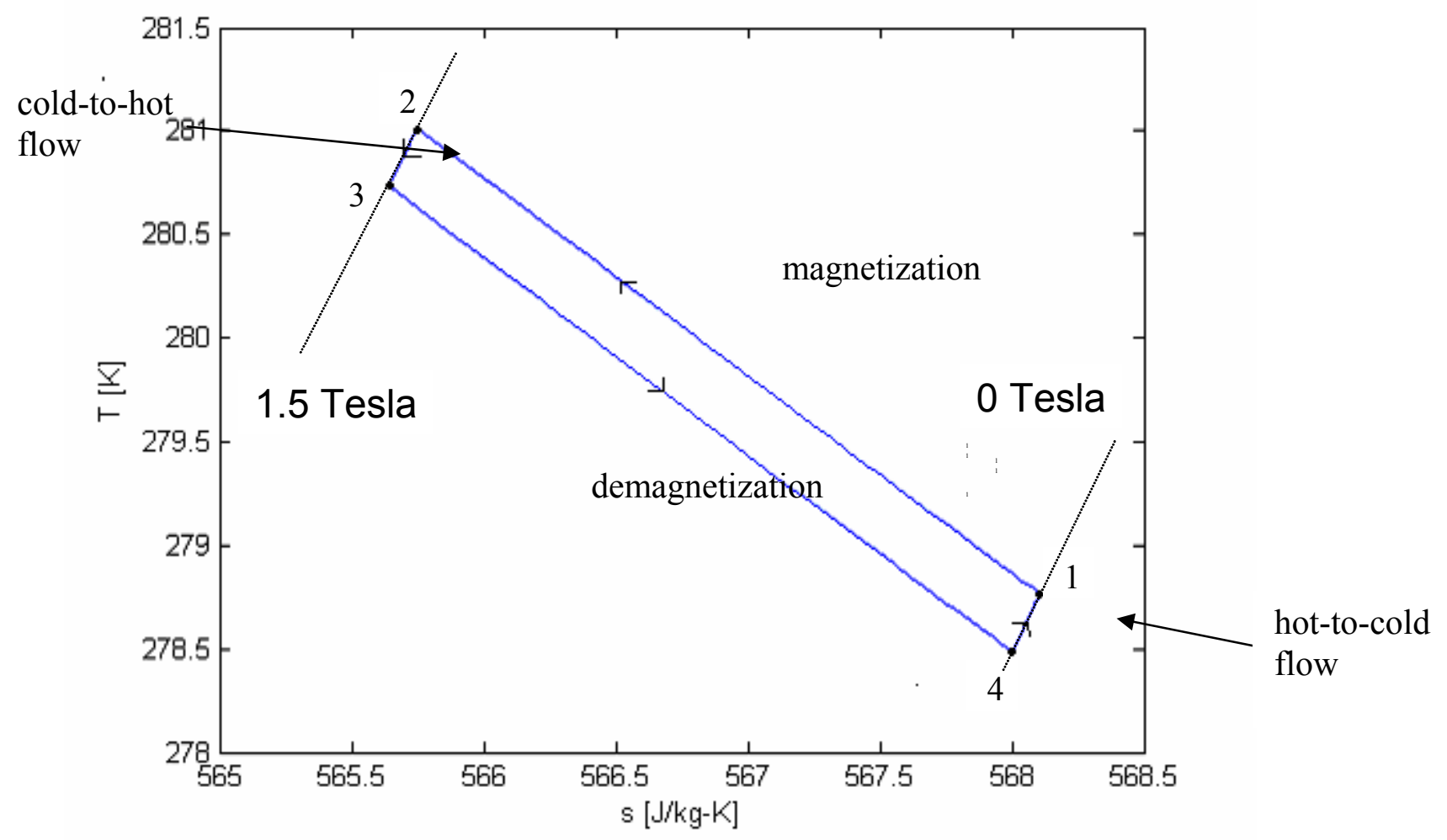

Figure 2.19. T-s Diagram of a Differential Regenerator Segment

The three methods described in this section are used to check that the model is behaving properly whenever a new feature is added or any change is made. For example, if a new 
working fluid is implemented, the model is checked to ensure that the fluid properties produce thermodynamically consistent results. When changes are made to the model, it is checked for errors and inconsistencies by comparing the results of $W_{\text {mag }}$ calculated independently using equations (2.67) and (2.70), the plot of the entropy flux is examined to ensure that entropy production is positive, and the $T$-s diagrams for the magnetic material in the regenerator are examined. 


\section{Chapter $3 \quad$ Passive Regenerator Test Results}

\subsection{Experimental Apparatus}

Astronautics has developed an experimental setup capable of measuring the performance of a passive regenerator. This experimental apparatus is valuable to this project in that it allows the investigation of the performance of a regenerator in which the heat capacity of the fluid is comparable to the heat capacity of the regenerator matrix; this is a situation that is not typical and therefore not well understood.

The experimental setup is shown in Figure 3.1. The experiment is designed to allow the cycle time, dwell ratio (the ratio of the portion of cycle with no flow to the total cycle time), the heater power, and the mass flow rate of the water to be varied. The pressure drop across the bed and the hot and cold reservoir temperatures seen by the bed are measured. The cold inlet water is provided from a constant-temperature bath and the heater power, $Q_{h, e x p}$, determines the steady state hot reservoir temperature. The heater power is a measure of the performance of the regenerator; a perfect regenerator would require no heater power to sustain an arbitrary hot reservoir temperature whereas a real regenerator suffers from a heat loss that is directly related to the regenerator ineffectiveness.

Experimental data were collected using this passive regenerator test facility with back-toback beds of packed stainless steel spheres under conditions that correspond to various values of dwell ratio, flow rates, reservoir temperatures, and cycle times. The experimental data were used to test the accuracy of two versions of the model: the 
lumped capacitance model and the modified lumped capacitance model. The lumped capacitance model assumes that the thermal effect of the fluid thermal capacitance can be considered by combining (i.e. lumping) the thermal capacitance of the fluid with the thermal capacitance of the regenerator bed material. The modified lumped capacitance model (described in section 2.7) applies an empirical correction factor to the heat transfer coefficient between the fluid and regenerator bed material to account for the reduction in performance that results from combining the fluid and regenerator capacitance. The model inputs used to predict the regenerator performance for each data point are given in Table 3.1; these values were chosen to match the conditions in the experiment.

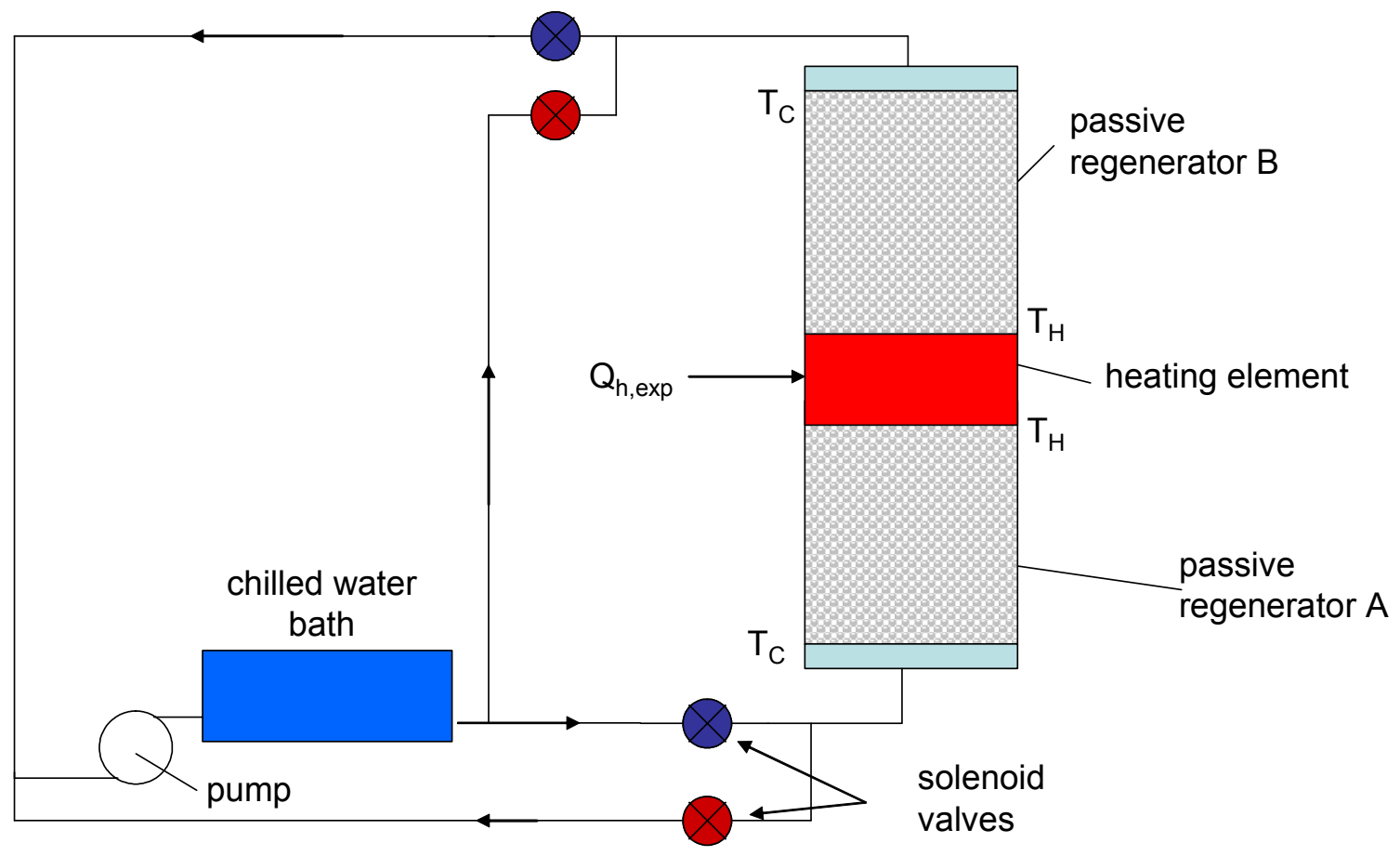

Figure 3.1. Schematic of the passive regenerator test setup 
Table 3.1. Model inputs used to match the passive regenerator test results

\begin{tabular}{|l|c|l|c|}
\hline \multicolumn{1}{|c|}{ Parameter } & Value & \multicolumn{1}{c|}{ Parameter } & Value \\
\hline bed length & $0.0982 \mathrm{~m}$ & average sphere size for packing & $0.389 \mathrm{~mm}$ \\
\hline bed cross section & $0.000459 \mathrm{~m}^{2}$ & regenerator material & $\begin{array}{c}\text { stainless steel } \\
\text { (constant prop) }\end{array}$ \\
\hline porosity & 0.358 & heat transfer fluid & $\begin{array}{c}\text { Water (constant } \\
\text { prop) }\end{array}$ \\
\hline maximum applied field & 0 Tesla & bed layering & single mat. \\
\hline
\end{tabular}

\subsection{Comparison of Model Predictions with Data}

\section{Lumped Capacitance Model}

The lumped capacitance model combines the heat capacity of the heat transfer fluid that is entrained in the void volume of the bed with the heat capacity of the regenerator material when solving the system governing equations. This assumption makes the model more stable and computationally efficient; however, it is a conservative assumption that under predicts the performance of a regenerator bed. For this study, the lumped capacitance model uses a numerical grid of 80 spatial steps and 120 times steps. Sensitivity studies have shown that this numerical grid is sufficient to obtain accurate results.

\section{Modified Lumped Capacitance Model}

The modified lumped capacitance model uses the method described in section 2.7 to correct for the lumped capacitance assumption. In the modified lumped capacitance model, the fluid to regenerator heat transfer is calculated using an augmented heat transfer coefficient. The modified lumped capacitance model is identical to the lumped capacitance model with the exception that the heat transfer in the bed is enhanced by a 
factor that depends on the ratio of the fluid thermal capacitance to the bed thermal capacitance.. The modified lumped capacitance model was run with the same numerical grid of 80 spatial steps and 120 times steps as the lumped capacitance model.

\section{$\underline{3.2 .1 \text { Results }}$}

Two experiments were run using the passive regenerator test apparatus at Astronautics' Madison facility. During the first set of tests (Set A), the hot and cold reservoirs were maintained at nearly constant temperatures while all other parameters were varied. During the second set of tests (Set B), the dwell ratio was held constant while the mass flow rate was varied; the reservoir temperatures also varied somewhat during these tests. Table 3.2 summarizes the test conditions and measured data for the Test Set A and also lists the predicted heater power based on the lumped capacitance and modified lumped capacitance models. In Table 3.2, the column labeled $\%$ ineff $H$ is the regenerator ineffectiveness based on the hot-side, $Q_{h, \text { exp }}$ is the experimental heater power, $Q_{h, L C}$ is the required heater power predicted by the lumped capacitance model, and $Q_{h, \text { cor }}$ is the heater power predicted by the modified lumped capacitance model. The heater power required is a measure of regenerator ineffectiveness, and higher heater powers indicate less efficient regeneration. 
Table 3.2. Experimentally measured and predicted passive regenerator results under conditions associated with Test Set A

\begin{tabular}{|c|c|c|c|c|c|c|c|c|c|c|}
\hline $\begin{array}{c}\text { flow rate } \\
{[\mathrm{kg} / \mathrm{s}]}\end{array}$ & $\begin{array}{c}\text { cycle time } \\
{[\mathrm{s}]}\end{array}$ & $\begin{array}{c}\text { dwell ratio } \\
{[\%]}\end{array}$ & $\begin{array}{c}\text { Tc } \\
{[\mathrm{K}]}\end{array}$ & $\begin{array}{c}\text { Th } \\
{[\mathrm{K}]}\end{array}$ & $\%$ ineff $\mathrm{H}$ & $\begin{array}{c}\mathrm{Q}_{\mathrm{h}, \exp } \\
{[\mathrm{W}]}\end{array}$ & $\begin{array}{c}\mathrm{Q}_{\mathrm{h}, \mathrm{LC}} \\
{[\mathrm{W}]}\end{array}$ & $\begin{array}{c}\text { Err }_{\mathrm{LC}} \\
{[\%]}\end{array}$ & $\begin{array}{c}Q_{\mathrm{h}, \text { cor }} \\
{[\mathrm{W}]}\end{array}$ & $\begin{array}{c}\text { Err }_{\text {cor }} \\
{[\%]}\end{array}$ \\
\hline 0.0088 & 2.027 & 33.5 & 297.2 & 317.1 & 2.7 & 13.1 & 12.4 & 5.1 & 6.4 & 51.0 \\
\hline 0.0179 & 1.024 & 33.8 & 297.4 & 317.2 & 2.2 & 22.0 & 33.6 & 52.8 & 16.9 & 23.1 \\
\hline 0.0352 & 0.522 & 33.8 & 297.2 & 316.9 & 1.8 & 34.3 & 88.5 & 157.9 & 46.9 & 36.7 \\
\hline 0.0090 & 4.036 & 33.4 & 297.7 & 317.4 & 3.3 & 16.2 & 13.8 & 14.8 & 7.3 & 54.9 \\
\hline 0.0177 & 2.049 & 33.1 & 297.5 & 317.7 & 2.5 & 24.8 & 37.2 & 49.9 & 19.7 & 20.6 \\
\hline 0.0352 & 1.026 & 33.7 & 297.4 & 317.2 & 2.3 & 45.0 & 94.6 & 110.3 & 51.5 & 14.5 \\
\hline 0.0088 & 2.028 & 33.5 & 297.1 & 306.8 & 2.1 & 5.1 & 6.1 & 20.5 & 3.1 & 38.7 \\
\hline 0.0176 & 1.024 & 33.7 & 297.1 & 306.8 & 1.9 & 9.1 & 16.3 & 78.4 & 8.3 & 9.2 \\
\hline 0.0342 & 0.523 & 33.8 & 297.1 & 306.8 & 1.9 & 17.7 & 42.7 & 141.4 & 22.2 & 25.5 \\
\hline 0.0182 & 1.024 & 33.8 & 297.2 & 317.3 & 1.8 & 18.4 & 34.9 & 90.1 & 18.0 & 1.9 \\
\hline 0.0147 & 1.025 & 17.3 & 297.2 & 317.2 & 1.6 & 16.8 & 32.2 & 91.7 & 16.5 & 1.8 \\
\hline 0.0130 & 1.024 & 9.3 & 297.2 & 317.1 & 1.5 & 15.0 & 29.0 & 93.2 & 14.8 & 1.4 \\
\hline 0.0176 & 1.024 & 33.7 & 297.3 & 317.2 & 1.9 & 18.3 & 32.9 & 79.3 & 17.0 & 7.4 \\
\hline 0.0143 & 1.025 & 17.3 & 297.2 & 317.3 & 1.6 & 15.8 & 31.0 & 96.2 & 15.9 & 0.6 \\
\hline 0.0131 & 1.024 & 9.2 & 297.2 & 317.1 & 1.5 & 14.9 & 29.6 & 99.1 & 15.2 & 2.3 \\
\hline
\end{tabular}

The experimental and predicted results for the lumped and modified lumped capacitance models are summarized in Figure 3.2 which illustrates the predicted heater power as a function of the measured heater power; note that a perfect model would predict results that fall on a line with a slope of 1.0 that intercepts the origin. As expected, the lumped capacitance model predicts values of $Q_{h}$ that are generally higher than the experimental results; this result verifies the conservatism of this model - the lumped capacitance model predicts lower regenerator efficiencies than are experimentally observed. The modified lumped capacitance model predicts more accurate results on average for this test, especially when flow rates are sufficiently high and dwell ratios are relatively low; note that the modified lumped capacitance model was derived for the condition of zero dwell time. 


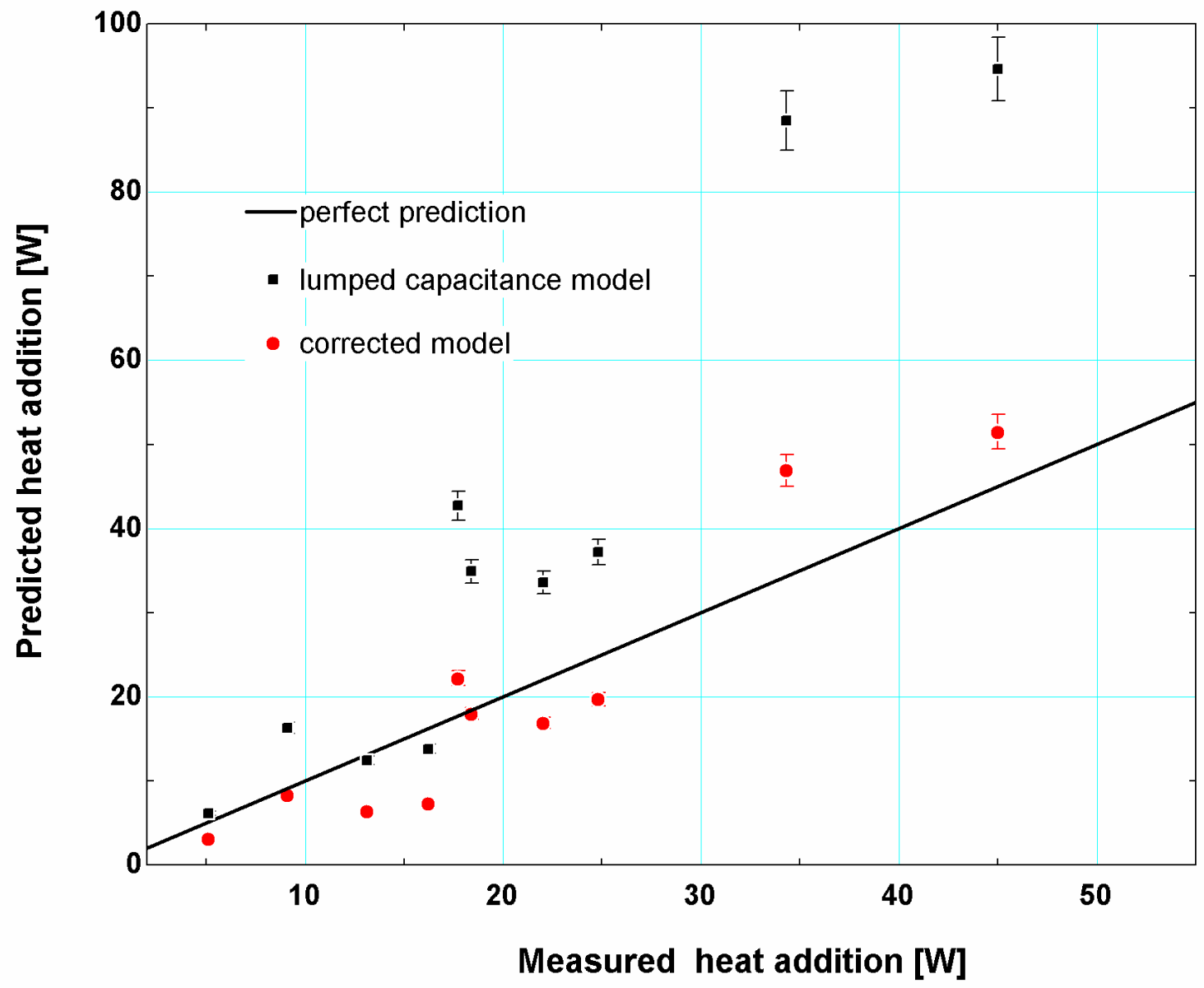

Figure 3.2. Predicted results from the lumped capacitance and modified lumped capacitance models as a function of the experimentally measured results for Test Set A.

Table 3.3 shows experimental data and predicted performance for Test Set B. In Table 3.3, $Q_{h, p r e d}$ is the heater power predicted by the modified lumped capacitance model, which represents the primary method used by the AMRR numerical model to account for the true, separated nature of the fluid and matrix heat capacity. Again, the heater power required is a measure of regenerator ineffectiveness, and higher heater powers indicate less efficient regeneration. 
Table 3.3. Experimentally measured and predicted passive regenerator results for Test

Set B

\begin{tabular}{|c|c|c|c|c|c|c|c|}
\hline $\begin{array}{c}\text { flow rate } \\
\text { [kg/s] }\end{array}$ & $\begin{array}{c}\text { cycle time } \\
\text { [s] }\end{array}$ & $\begin{array}{c}\text { dwell ratio } \\
{[\%]}\end{array}$ & $\begin{array}{l}\text { Tc } \\
{[\mathrm{K}]}\end{array}$ & $\begin{array}{l}\text { Th } \\
{[\mathrm{K}]}\end{array}$ & $\begin{array}{c}Q_{h, \exp } \\
{[W]}\end{array}$ & $\begin{array}{c}Q_{h, \text { pred }} \\
{[W]}\end{array}$ & $\begin{array}{c}\text { Err }_{\text {pred }} \\
{[\%]}\end{array}$ \\
\hline 0.0067 & 1.025 & 10.9 & 273.15 & 315.31 & 10.3 & 4.7 & 54.5 \\
\hline 0.0091 & 1.025 & 10.9 & 273.15 & 321.59 & 11.6 & 10.0 & 14.0 \\
\hline 0.0113 & 1.025 & 10.9 & 273.15 & 316.48 & 11.6 & 11.1 & 4.5 \\
\hline 0.0133 & 5 & 10.9 & 273.15 & 313.05 & 12.2 & 11.7 & 4.4 \\
\hline 0.0183 & 1.025 & 10.9 & 273.15 & 315.18 & 20.6 & 22.0 & 7.0 \\
\hline 0.0234 & 1.025 & 10.9 & 273.15 & 312.37 & 24.3 & 27.8 & 14.5 \\
\hline 0.0264 & 1.025 & 11.0 & 273.15 & 314.64 & 32.6 & 39.2 & 20.4 \\
\hline 0.0308 & 1.025 & 10.9 & 273.15 & 314.29 & 40.3 & 50.1 & 24.4 \\
\hline
\end{tabular}

The experimental measurements and predicted results for the lumped capacitance and the modified lumped capacitance models are summarized in Figure 3.3 which again illustrates the predicted heater power as a function of the measured power. Again, the lumped capacitance model predicts values of $Q_{h}$ that are generally much higher than the experimental results. The modified lumped capacitance model predicts more accurate results. 


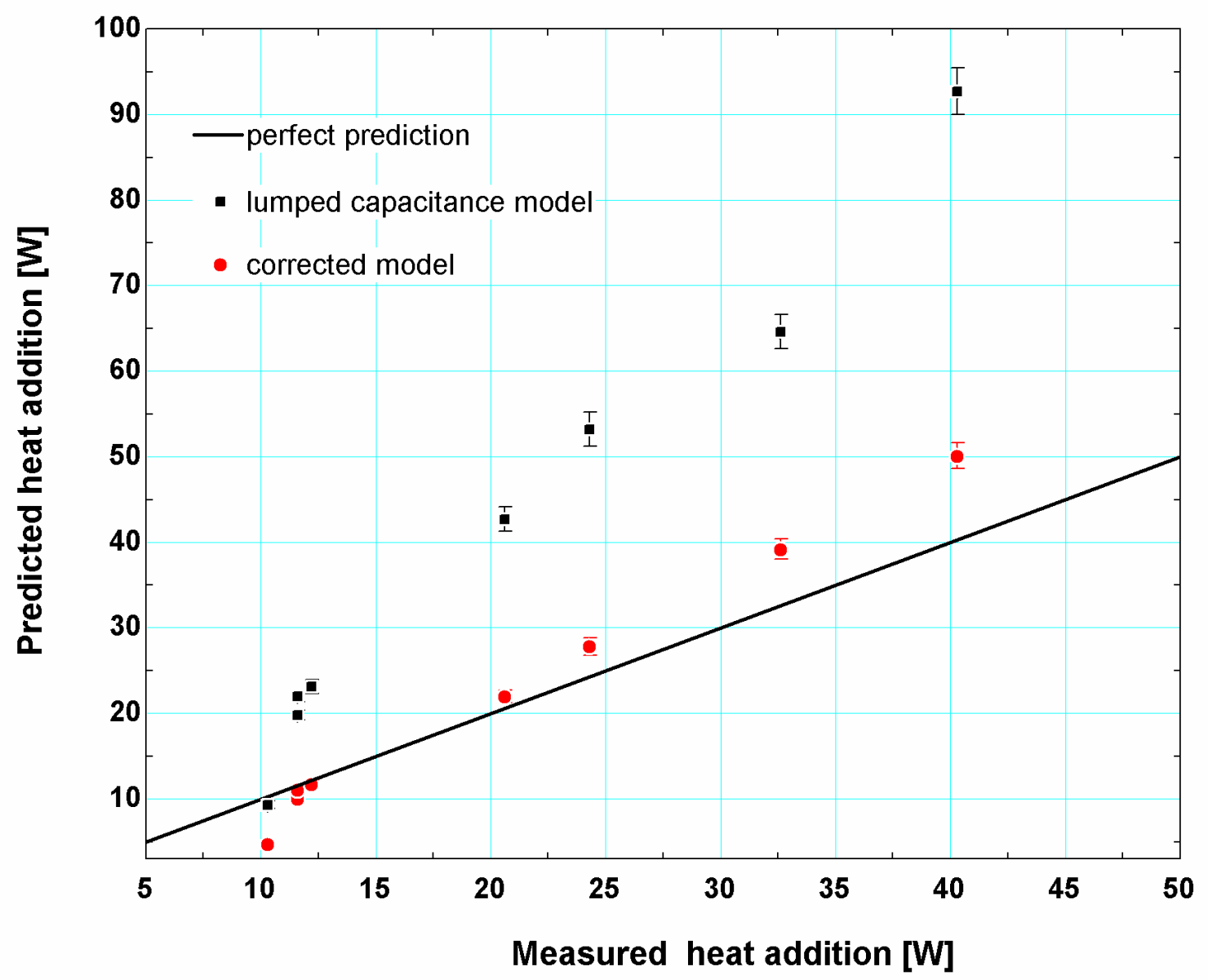

Figure 3.3. Predicted results from the lumped capacitance and modified lumped capacitance models as a function of the experimentally measured results for Test Set B.

Figures 3.2 and 3.3 show that the modified lumped capacitance model (which uses an enhanced heat transfer coefficient to account for the lumped fluid capacity) is a viable, approximate method for correcting for the lumped capacitance assumption in the passive regenerator case and likely also represents the most attractive means of accounting for this phenomenon in the magnetic refrigeration model. Two experiments were conducted at Astronautics and they support the fact that the corrected lumped capacitance model is 
capable of accurately predicting passive regenerator performance at lower dwell ratios and mass flow rates that are sufficiently high. At high dwell ratios and very low mass flow rates, the model does not agree well with experimental results, possibly because of high experimental uncertainty at these conditions as well as inadequacy of the model with nonzero dwell ratio. It is possible to augment the model in order to account for nonzero dwell times as well as other effects.

\subsubsection{Experimental Uncertainty}

An error analysis of the passive regenerator test setup shown in Figure 3.1 is described in this section. A listing of the instrumentation used to collect the experimental data and specify the model inputs as well as their associated uncertainty is shown in Table 3.4.

Table 3.4. Passive regenerator test instrumentation and model inputs and associated uncertainty

\begin{tabular}{|l|c|}
\hline \multicolumn{1}{|c|}{ Instrument } & Uncertainty \\
\hline Data Acquisition time step & $10 \mu \mathrm{s}$ \\
\hline CAP SD-1 Solenoid timing & $25 \mu \mathrm{s}$ \\
\hline YSI 44033 Thermistor & $0.1{ }^{\circ} \mathrm{C}$ \\
\hline Flow Technology FT Series Turbine Flowmeters & $.00016 \mathrm{~kg} / \mathrm{s}$ \\
\hline Heater voltage & $0.015 \%$ of reading \\
\hline Heater current & $0.015 \%$ of reading \\
\hline Sphere diameter & $7 \mu \mathrm{m}$ \\
\hline Regenerator bed dimensions & $0.05 \mathrm{~mm}$ \\
\hline
\end{tabular}

In order to determine the effect of measurement uncertainty on the predicted values, each measured input to the model (e.g., the sphere diameter) was increased by the uncertainty 
in each measurement separately. The resulting change in the predicted performance was assumed to be the prediction uncertainty related to the uncertainty in that given input. In this way, a model uncertainty related to each of the measurement uncertainties was estimated. To determine the total uncertainty in the model predictions, the root sum squared of all the calculated prediction uncertainties was taken. The results are indicated as the error bars evident in Figures 3.2 through 3.4. 


\section{Chapter $4 \quad$ System Modeling}

\subsection{Auxiliary Equipment}

The electric motor used to rotate the regenerator and the pump that provides the desired fluid mass flow rate are both modeled by assigning an overall efficiency relative to an ideal thermodynamic process to these components. Using this approach, the power input to the pump, $\dot{W}_{\text {pump }}$, is:

$$
\dot{W}_{\text {pump }}=\frac{\dot{m}_{f} \Delta P}{\eta_{\text {pump }} \rho_{f}}
$$

where $\eta_{\text {pump }}$ is the overall efficiency of the pump, $\Delta P$ is the pressure drop across the bed, $\rho_{f}$ is the fluid density, and $\dot{m}_{f}$ is the fluid mass flow rate. Similarly, the power to run the electric motor is based on the work input to the regenerator required by the magnet from section 2.8.

$$
\dot{W}_{\text {mag }}=\frac{W_{\text {mag }}}{\tau \eta_{\text {motor }}}
$$

where $W_{\text {mag }}$ is the magnetic work done on the regenerator per cycle, $\tau$ is the cycle time, and $\eta_{\text {motor }}$ is the overall motor efficiency.

\subsection{Grid Size Determination}

The model uses a numerical grid of $m$ time steps and $n$ spatial steps to generate temperature profiles with a relaxation tolerance of $0.005 \mathrm{~K}$. The parameter used to evaluate the accuracy of the model with respect to numerical grid size is the predicted refrigeration load. The model was run with a constant Curie temperature (i.e. non- 
layered) bed and with varying values of $m$ and $n$. The results are shown in Figure 4.1.

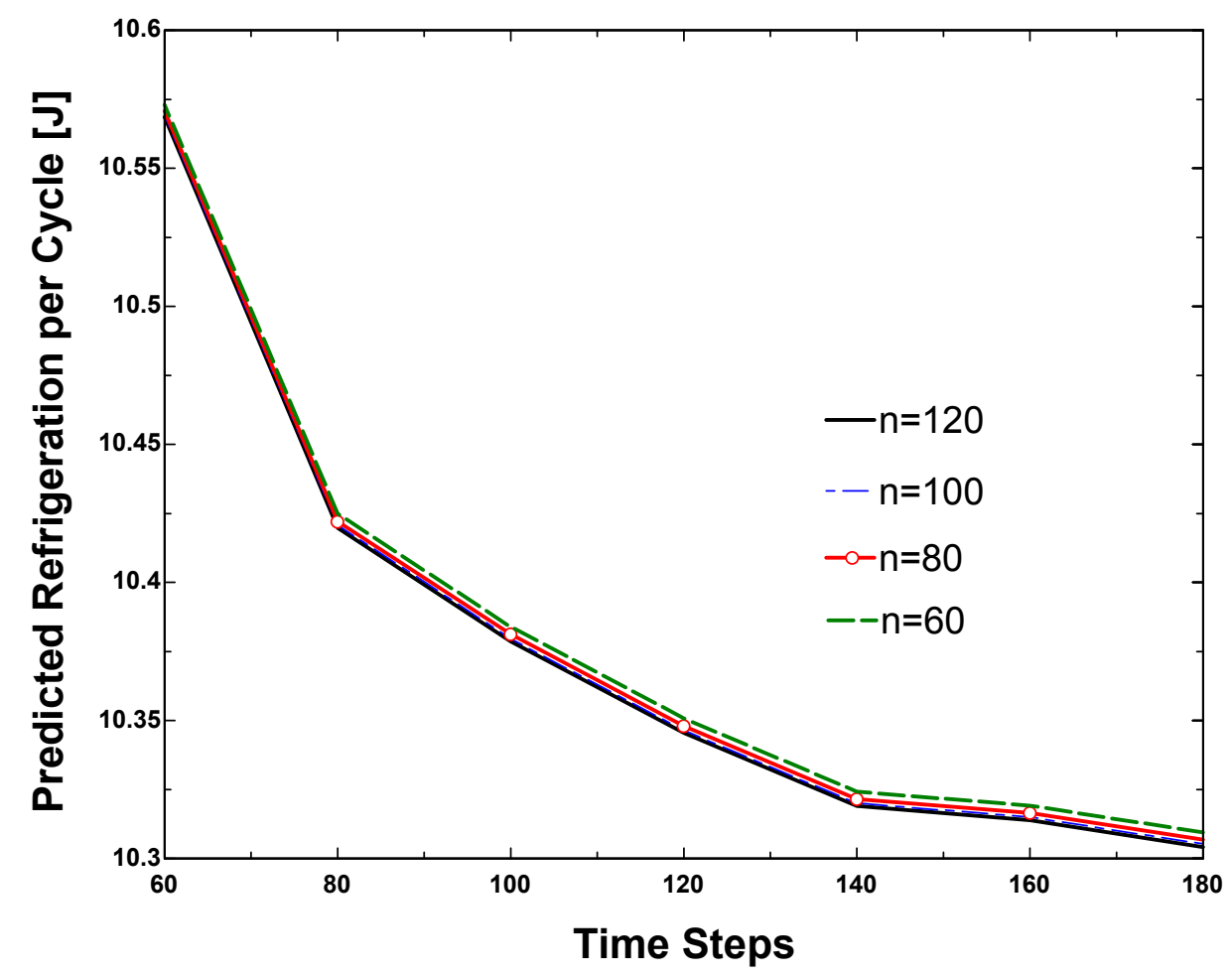

Figure 4.1. Predicted refrigeration per cycle as a function of the number of time steps $(m)$ for various values of the number of axial steps $(n)$.

Figure 4.1 indicates that the predicted refrigeration per cycle has a weak dependence on the number of spatial steps, $n$. For example, the lines associated with $n=120$ and $n=100$ are essentially indistinguishable; therefore, the results at $n=120$ are assumed to be nominally equal to results with an infinite number of spatial steps. However, a stronger dependence on the number of time steps is observed. The refrigeration with an infinite number of time and spatial steps (i.e., the true refrigeration) may be approximately 
extrapolated using $n=120$ data from Figure 4.1. Figure 4.2 shows a curve fit of the predicted refrigeration per cycle as a function of time steps, $m$, shown in Eq. (4.3).

$$
\text { refrigeration }=10.2999-\frac{6.26432}{m}+\frac{1334.12}{m^{2}}
$$

As $m$ approaches infinity, the curve fit indicates that the refrigeration will limit to 10.2999 J. This extrapolation approach is similar to the Richardson extrapolation technique described in some textbooks, for example Jaluria (2002). The "correct" value of refrigeration obtained using this extrapolation technique was subsequently used to determine the error in the model output for each value of numerical grid.

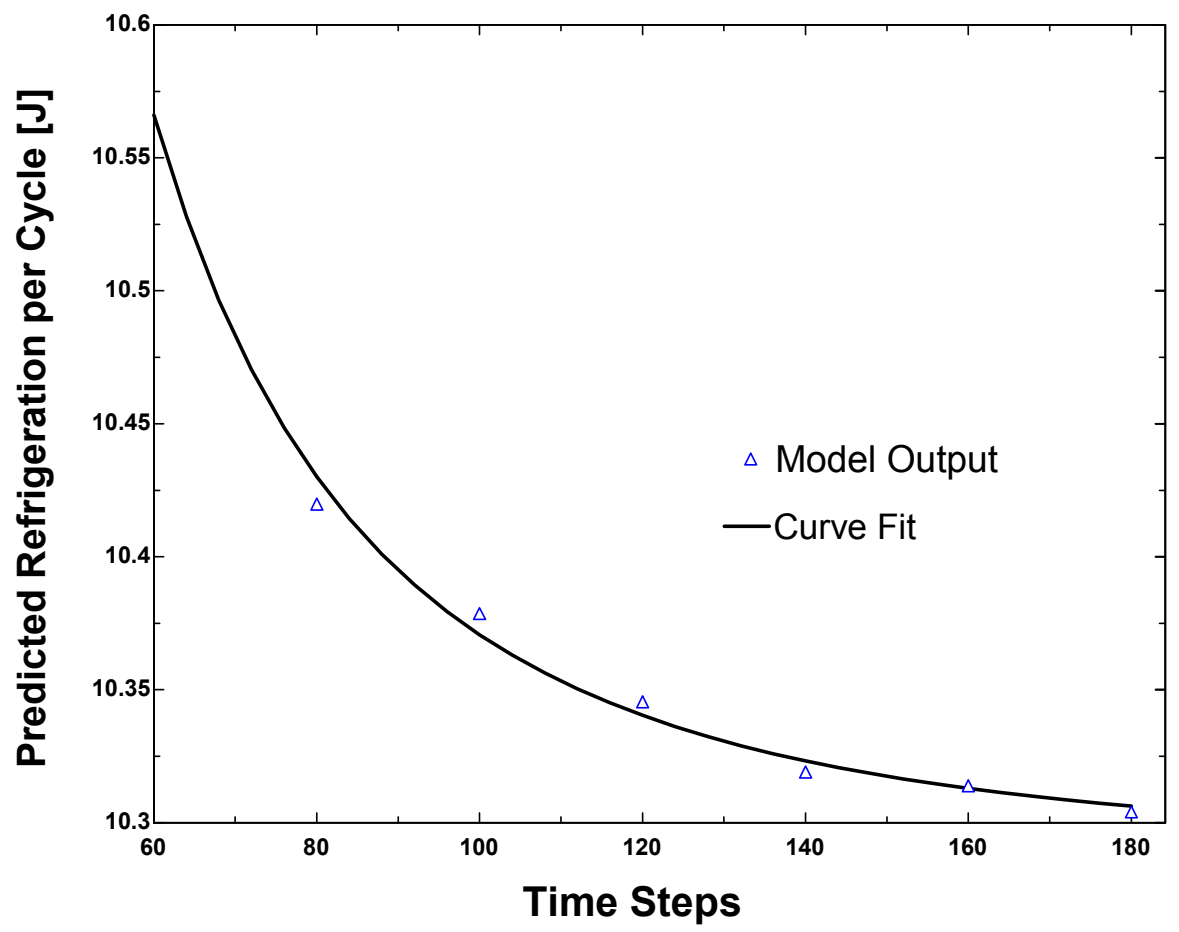

Figure 4.2. Predicted refrigeration per cycle as a function of number of time steps $(m)$ used in the simulation for a non-layered bed and the curve fit used to extrapolate to the true solution. 
This investigation of the grid dependence indicates that satisfactory accuracy may be obtained using a numerical grid consisting of 60 spatial steps and 120 time steps. This size grid was selected based on an optimal balance between practical computational time and a reasonable numerical error, approximately $0.5 \%$ of refrigeration per cycle. The same procedure was used to determine the optimal grid size for a layered bed and the associated plot is shown in Figure 4.3.

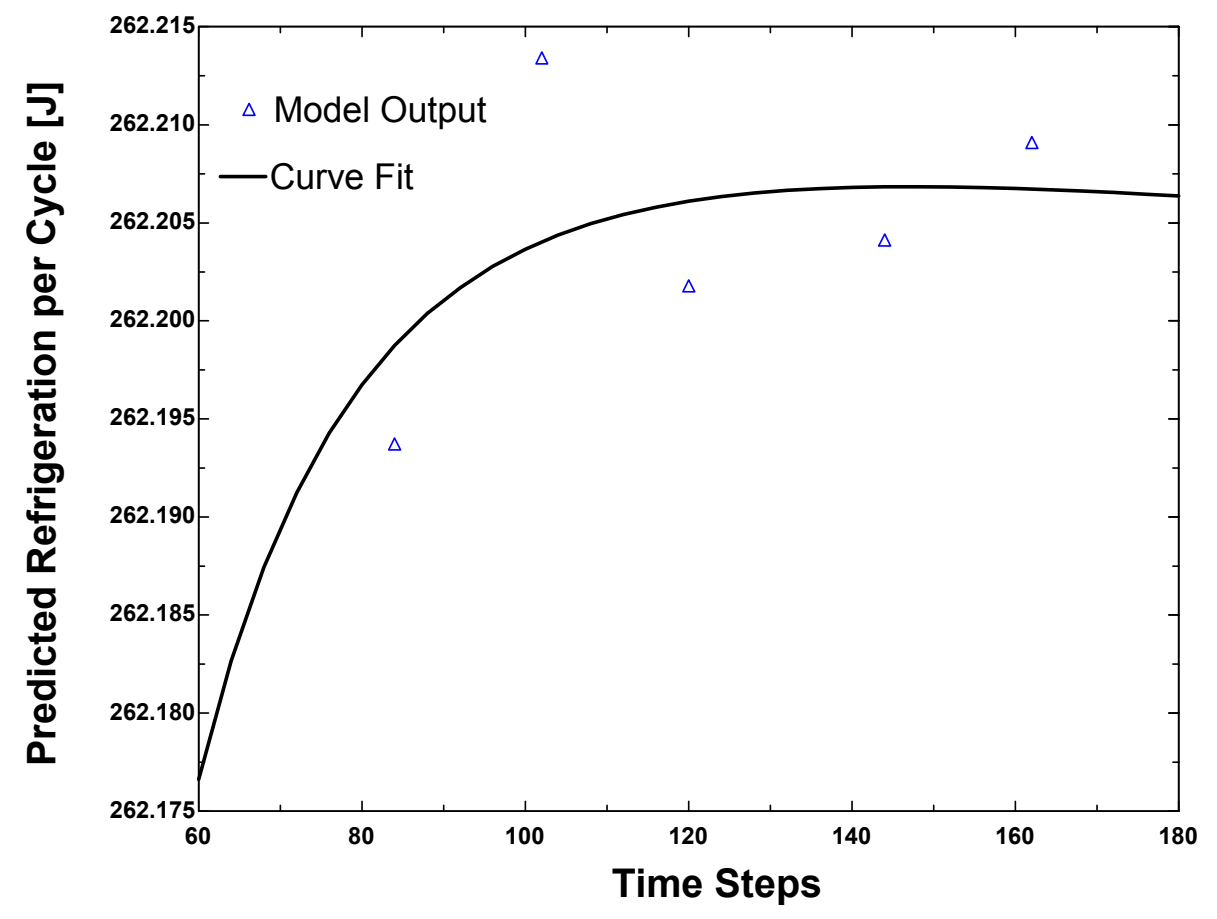

Figure 4.3. Refrigeration per cycle as a function of number of time steps $(m)$ for a layered bed

Results for a layered bed show weaker dependence on the number of timesteps than the results for a non-layered bed did, as evident by the extremely narrow scale in Figure 4.3 compared to Figure 4.2. As a result of this weak dependence, a smaller number of 
timesteps can be used without sacrificing accuracy. Therefore, a grid consisting of 60 spatial steps to 84 time steps was selected for a layered bed.

\subsection{Optimization Technique}

\subsubsection{Selection of Mass Flow Rate}

Magnetic coolers inherently have a variable cooling capacity which is dependent on the volume of the regenerator, bed length to cross sectional area (aspect ratio), and the heat transfer fluid flow rate. In order to design for a particular application, it is necessary to determine the fluid mass flow rate that must be used to achieve the cooling capacity required by the application given a regenerator bed geometry. For a regenerator of a fixed length and diameter, the functional dependence of the refrigeration capacity as a function of mass flow rate of water may be determined. Figure 4.4 illustrates the refrigeration capacity and COP for a typical AMR system using a regenerator of a fixed volume (25 L) and aspect ratio $(L / d=0.34)$. The results are shown both for a regenerator consisting of a single magnetic material alloy (i.e. a non-layered bed) as well as for a layered regenerator bed. The layered regenerator has a Curie temperature that varies linearly along the bed from the AMR hot reservoir temperature to the cold reservoir temperature, and the nonlayered regenerator contains a constant Curie temperature magnetic material that is set at the average of the hot and the cold reservoir temperatures. For each mass flow rate and refrigeration capacity, there is a corresponding value of the coefficient-of-performance, COP, also shown in Figure 4.4. The COP curve of the non-layered bed lies almost directly on top of the COP curve of the layered bed when these are plotted against the water mass flow rate, indicating that a layered bed is capable of producing a higher 
cooling capacity than a non-layered bed at approximately the same efficiency. COP reported in Figure 4.4 includes power to run the electric motor and the pump.

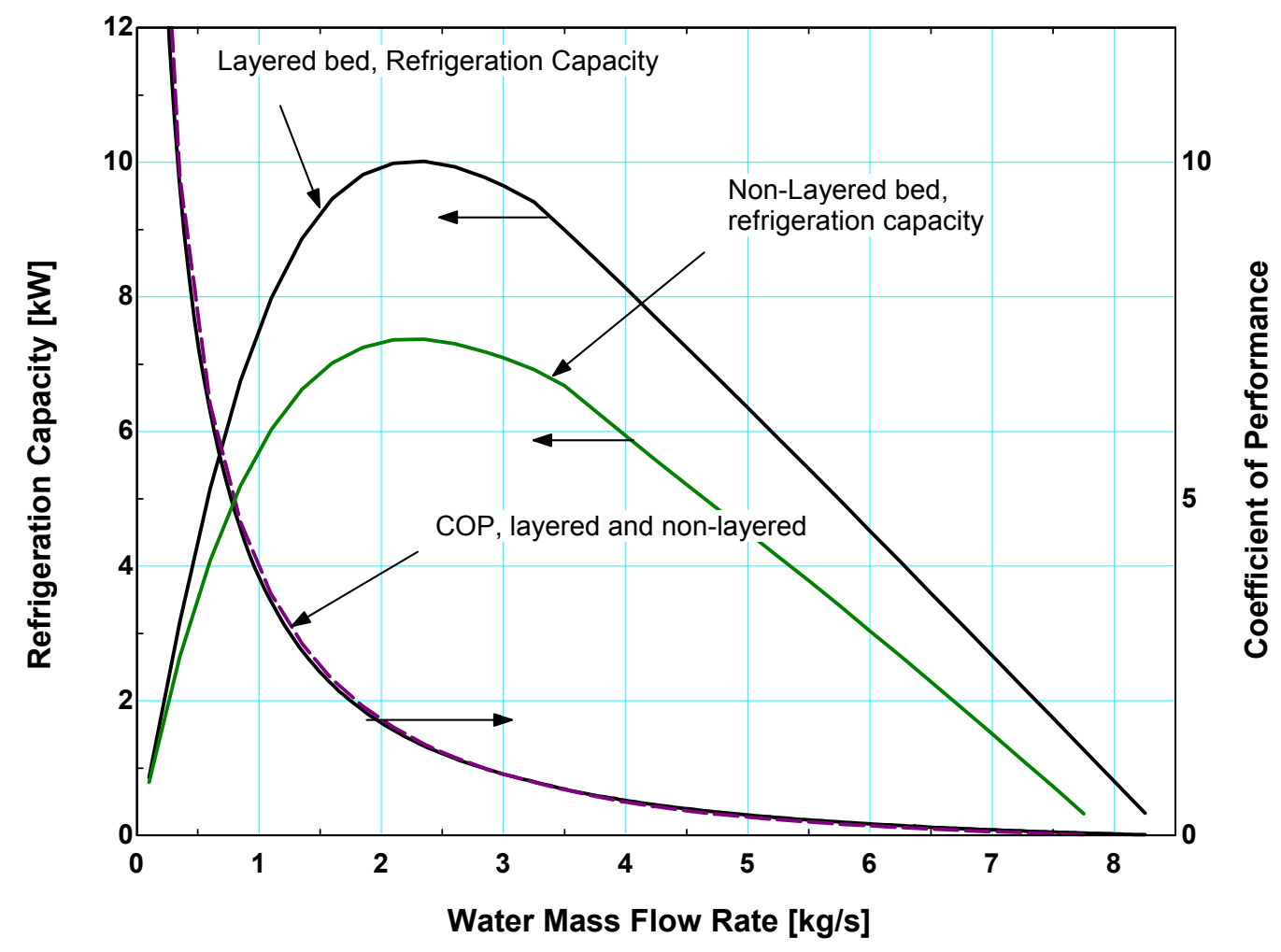

Figure 4.4. Refrigeration capacity and COP of a $25 \mathrm{~L}$ layered and non-layered bed with an aspect ratio of 0.34 as a function of the water mass flow rate.

Notice that if a horizontal line were drawn on Figure 4.4 corresponding to a constant refrigeration capacity of $6 \mathrm{~kW}$, it would intersect the capacity curves for the layered and non-layered beds at two points, each corresponding to a different mass flow rate. At each of these mass flow rates there is a corresponding coefficient of performance (COP); it is always true that the lower mass flow rate corresponds to a higher COP. The higher mass flow rate operating condition corresponds to an over-loaded bed; the potential 
refrigeration is high due to the high mass flow rate but the losses related to the mass flow rate are also large, hence the moderate capacity and low COP. At the lower mass flow rate the bed is appropriately loaded and the COP is higher. Therefore, for each type of bed configuration it is possible to produce a specified refrigeration capacity using one of two possible mass flow rates. The lower mass flow rate operating point with the higher COP is chosen for all following results. The procedure for determining the mass flow rate corresponding to a given refrigeration capacity has been automated. The AMR model is run over a range of fluid mass flow rates; the values of cooling capacity and COP are calculated for each value of mass flow rate. These data are interpolated using a piecewise cubic hermite interpolating polynomial in order to select the mass flow rate required to produce the desired cooling capacity; the lower mass flow rate is selected by constraining the solution to those values where the slope of capacity against mass flow rate is positive.

\subsubsection{Selection of Aspect Ratio}

The effect of regenerator geometry at a given regenerator volume was studied by choosing a total regenerator volume and varying the aspect ratio $(L / d)$. All other inputs to the model are identical to those that were used to generate Figure 4.4. For each regenerator geometry, the mass flow rate was selected in order to obtain $6 \mathrm{~kW}$ of refrigeration capacity using the procedure described in the previous section. Figure 4.5 illustrates the COP as a function of the aspect ratio for a layered regenerator bed. The scale of the figure is limited because there are some aspect ratio/volume combinations 
that are not capable of producing the desired cooling capacity for any value of mass flow rate.

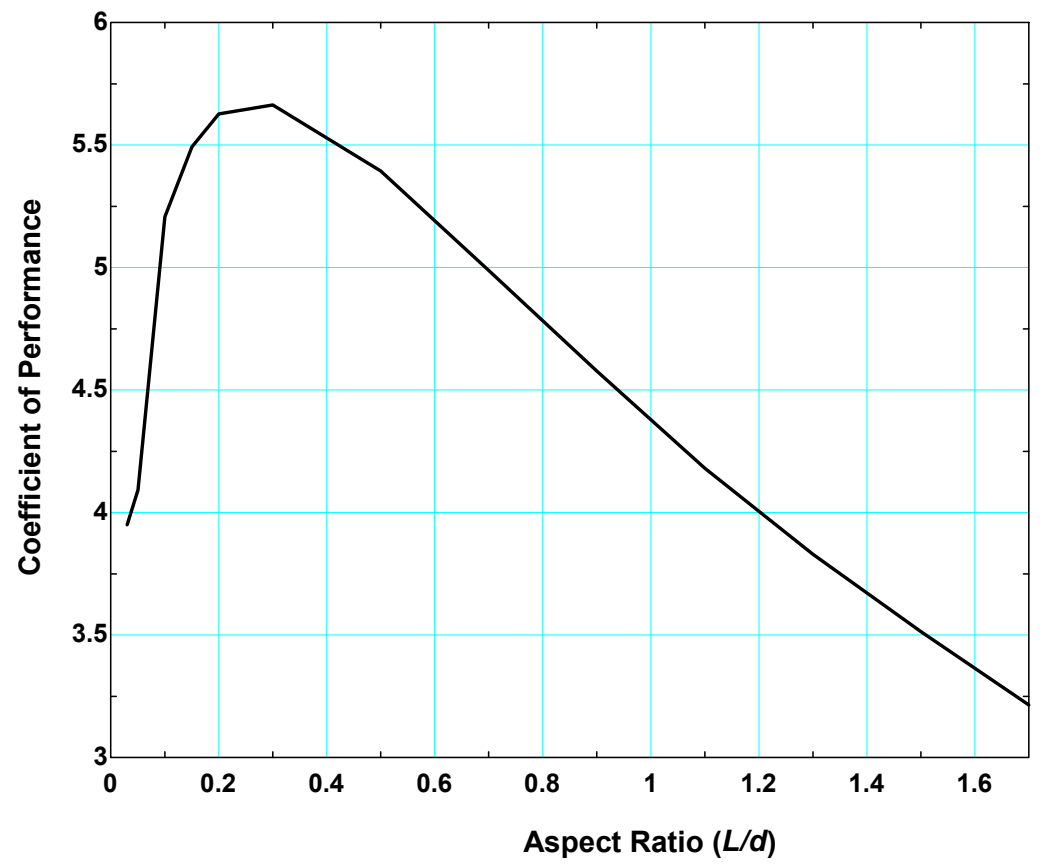

Figure 4.5. COP of a layered bed producing function of the aspect ratio.

Figure 4.5 shows that there exists an optimal aspect ratio which maximize the COP of the bed; lower aspect ratios result in excessive conduction losses and higher aspect ratios result in excessive pumping losses. This optimization technique is used to choose the combination of the optimum mass flow rate and aspect ratio of an AMRR of specified regenerator volume and cooling capacity for various applications. 
90

\subsection{Space Conditioning Application}

\subsubsection{Heat Exchanger Modeling}

In order to accurately model the performance of an AMRR in a space conditioning application it is necessary to adequately account for the performance of the heat exchangers which provide the thermal communication between the indoor air and the refrigerator (the cold heat exchanger) and the outdoor air and the refrigerator (the hot heat exchanger). The hot heat exchanger, the equivalent of the condenser, is modeled as a cross flow air-to-liquid heat exchanger for space cooling applications. The overall heat transfer coefficient, $U A_{H}$, and mass flow rate of air through the hot heat exchanger are assigned values that are reasonable for an equivalent vapor compression air-conditioning system. Since the temperature change across the heat exchanger is small, the specific heat of the fluid is assumed to be constant allowing conventional effectiveness-NTU equations to be used. Figure 4.6 shows a schematic of the hot heat exchanger. 


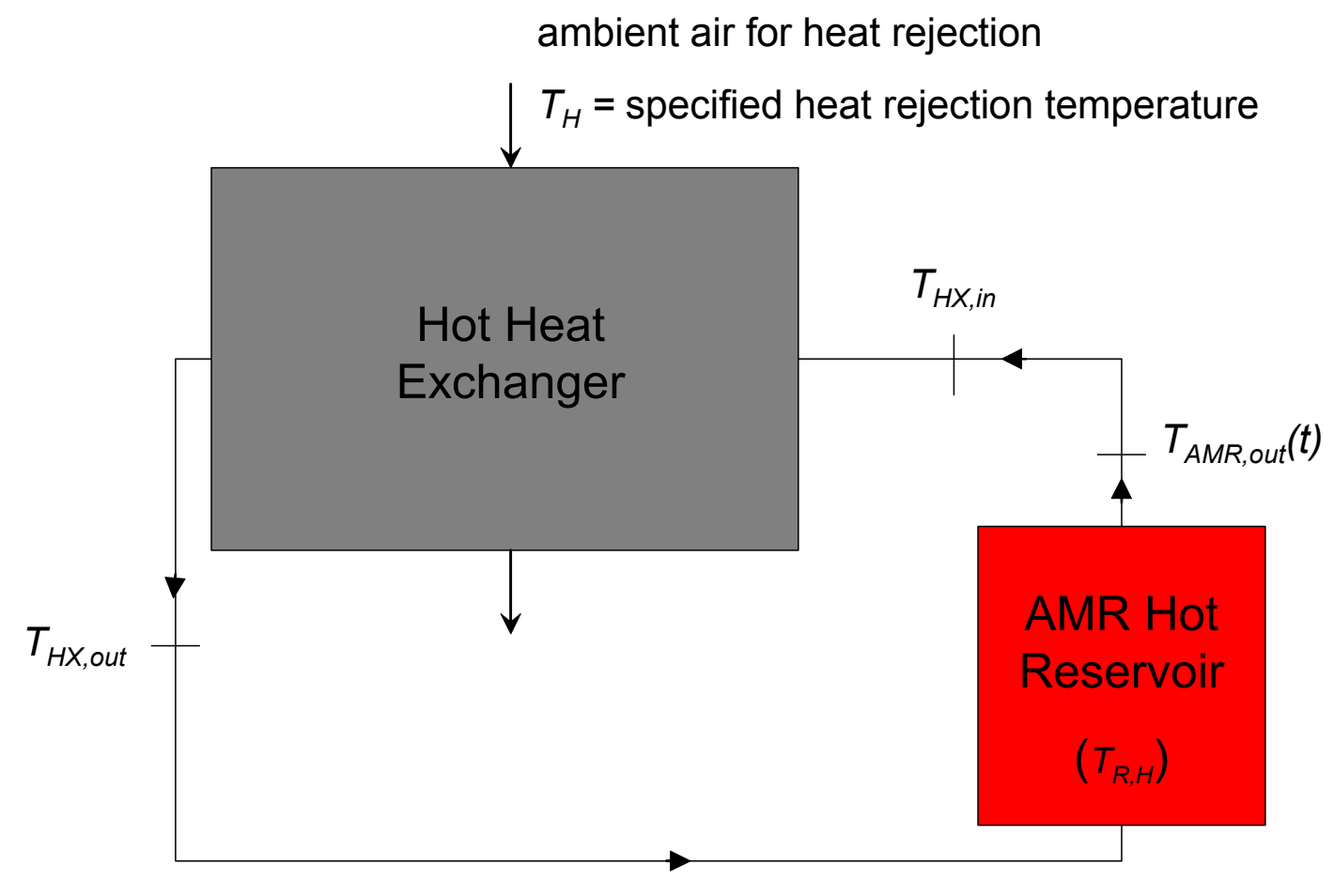

Figure 4.6. Hot heat exchanger schematic

The fluid entering the hot end of the AMR is assumed to be at a constant, hot reservoir temperature $T_{R, H}$. During the cold-to-hot flow process, fluid leaves the hot end of the AMR bed with temperature $T_{A M R, \text { out }}(t)$, which varies with time in a manner predicted by the model. The fluid exiting the AMR is assumed to mix fully and therefore enters the hot heat exchanger at a constant temperature $T_{H X, i n, H}$ which is higher than the hot reservoir temperature, $T_{R, H}$, in order to drive the heat transfer. $T_{H X, i n, H}$ is the enthalpy average temperature in time of the fluid leaving the hot end of the AMR. Air from the surroundings is used as a heat transfer medium and enters the heat exchanger at $T_{H}$, the heat rejection temperature specified by ARI. 
The maximum possible heat transfer rate in the hot heat exchanger $\left(\dot{q}_{\max , H}\right)$ is calculated.

$$
\dot{q}_{\max , H}=C_{\min , H}\left(T_{H X, i n, H}-T_{H}\right)
$$

where $C_{\min , H}$ is the minimum of the air side and fluid side heat capacity rates. To determine the effectiveness of the hot heat exchanger, the number of heat transfer units, $N T U_{H}$, for the hot side is calculated according to:

$$
N T U_{H}=\frac{U A_{H}}{C_{\min , H}}
$$

The hot heat exchanger effectiveness, $\varepsilon_{H}$, is calculated using the relationship given by Incropera and DeWitt (1996) for a cross flow heat exchanger with both fluids unmixed.

$$
\varepsilon_{H}=1-\exp \left[\left(\frac{1}{C_{r, H}}\right)\left(N T U_{H}\right)^{0.22} \cdot\left(\exp \left[-C_{r, H}\left(N T U_{H}\right)^{0.78}\right]-1\right)\right]
$$

where $C_{r, H}$ is the heat capacity ratio in the hot heat exchanger. The actual heat transfer in the hot heat exchanger $\left(\dot{q}_{H}\right.$ is calculated using the effectiveness.

$$
\dot{q}_{H}=\varepsilon_{H} \cdot \dot{q}_{\max , H}
$$

The enthalpy of the heat transfer fluid leaving the hot heat exchanger, $h_{H X, \text { out } H}$ is calculated from an energy balance:

$$
h_{H X, \text { out }, H}=h_{H X, i n, H}-\frac{\dot{q}_{H}}{\dot{m}_{f}}
$$

where $h_{H X, i n, H}$ is the enthalpy entering the hot heat exchanger. The temperatures can be obtained from the calculated enthalpies. 
In this thesis, the heat rejection temperature, $T_{H}$, is taken as the air temperature entering the hot heat exchanger, and the cold reservoir temperature, $T_{C}$, is the air temperature entering the cold heat exchanger. Although the hot and cold air temperatures are specified in the model, the temperature of the AMR hot and cold reservoirs, $T_{R, H}$ and $T_{R, C}$, are not directly specified and therefore must determined by iteration. The model assigns initial guess values to the hot and cold reservoirs of the AMR, $T_{R, C}^{*}$ and $T_{R, H}^{*}$.

$$
\begin{aligned}
& T_{R, H}^{*}=T_{H}+2 \\
& T_{R, C}^{*}=T_{C}-2
\end{aligned}
$$

where $T_{C}$ is the ARI specified temperature of the cold air supplied to the refrigeration application, and $T_{H}$ is temperature of the hot air supplied for heat rejection.

The numerical AMR model is exercised using the AMR reservoir guess values to obtain the temperature of the fluid leaving each reservoir. The equations described above can then be used to determine the temperature of the fluids leaving the hot and cold heat exchangers. The absolute value of the error between the guess values and the calculated values of these temperatures is determined. If the maximum error is less than a specified relaxation constant, the process is complete. If not, the guess values are updated as shown in equation (4.10) and used in a subsequent iteration. The results of the heat exchanger model are checked by verifying that the heat transfer between the fluid and the air is equal to the enthalpy flux per unit time at the corresponding end of the regenerator. A flow chart that summarizes the process is shown in Figure 4.7. 


$$
\begin{aligned}
& T_{R, H}^{*_{+}}=T_{H X, \text { out }, H} \\
& T_{R, C}^{*_{+}}=T_{H X, \text { out }, C}
\end{aligned}
$$

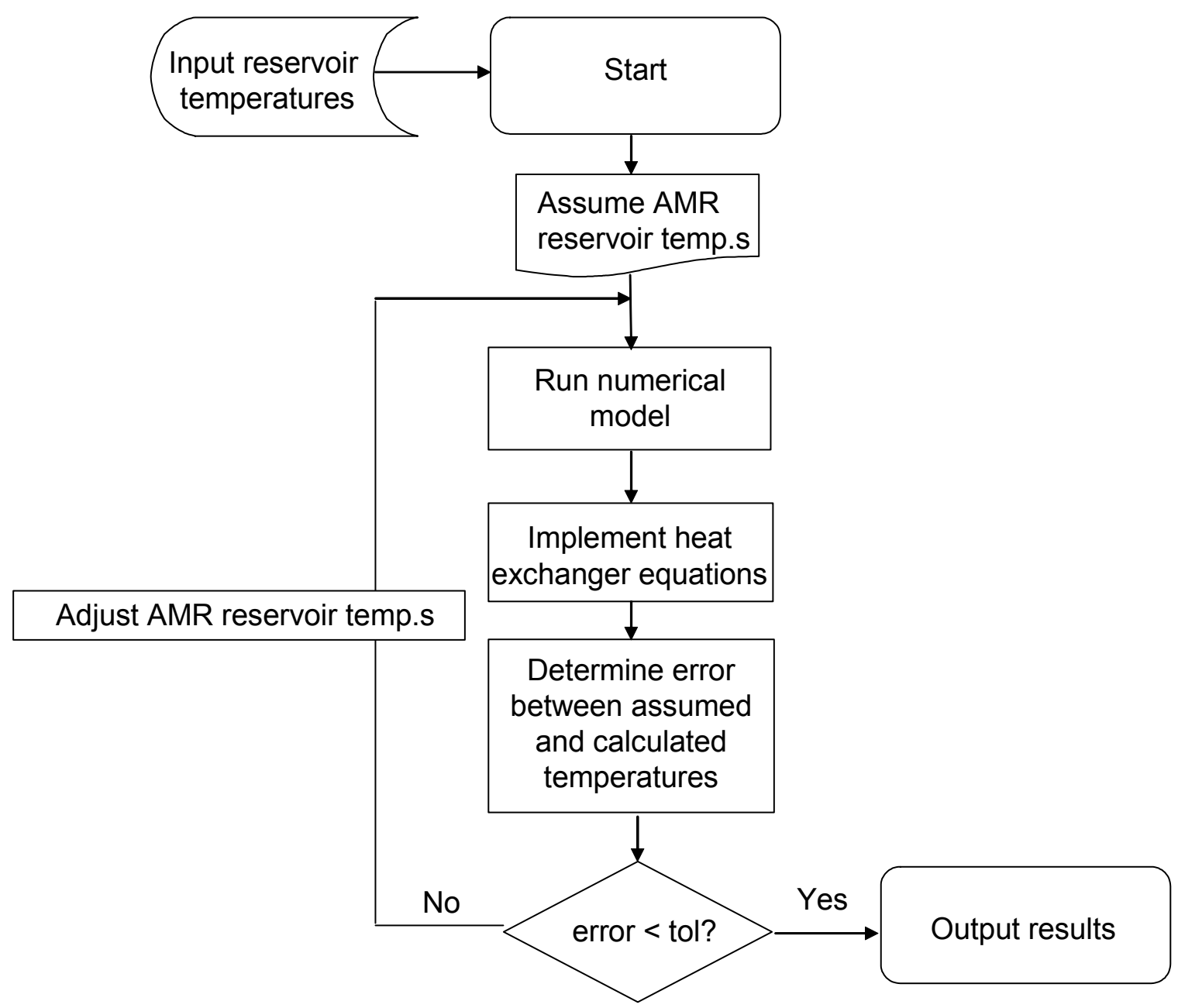

Figure 4.7. Heat exchanger model flow chart

Because a significant portion of the total heat transfer is related to a latent load in most evaporators within residential air conditioning systems, it is important to model this effect in the AMRR configuration. Braun et al. (1989) present a method to determine the heat exchanger effectiveness that is similar to the $\varepsilon-N T U$ method using a heat transfer 
analogy. To perform the analysis, the coil is assumed to be uniformly wet and the air is assigned a specific heat, $c_{s}$, based on the entrance and exit enthalpies divided by the temperature drop across the heat exchanger, as shown in Eq. (4.11).

$$
c_{s}=\frac{h_{a, s a t}\left(T_{H X, i n, C}\right)-h_{a, s a t}\left(T_{H X, o u t, C}\right)}{T_{H X, i n, C}-T_{H X, o u t, C}}
$$

where $T_{H X, i n, C}$ and $T_{H X, o u t, C}$ are the enthalpy averaged cold heat exchanger inlet and outlet liquid temperatures and $h_{a, s a t}$ is the enthalpy of saturated moist air. When using this method, the effectiveness of the heat exchanger, $\varepsilon_{C}$, is a function of $m^{*}$ and $N T U^{*}$, where $m^{*}$ is defined as

$$
m^{*}=\frac{\dot{m}_{a} c_{s}}{\dot{m}_{f} c_{f}}
$$

when the air side capacitance is less than the water side capacitance. The term $m^{*}$ is equivalent to the capacitance ratio in a sensible heat exchanger, $\dot{m}_{a}$ and $\dot{m}_{f}$ are the mass flow rates of the air and liquid, and $c_{f}$ is the specific heat of the liquid. The other variable needed to calculate efficiency, $N T U^{*}$, is given below.

$$
N T U^{*}=1.33 \frac{U^{*} A_{a}}{\dot{m}_{a}}
$$

where $A_{a}$ is the air side heat exchanger area. $N T U^{*}$ in Equation (4.13) has been increased by a factor of 1.33 over the value suggested by Braun et al. This is because heat exchanger performance predictions made without the 1.33 factor agreed well with the DOE/ORNL Heat Pump Design Model Mark V, which was first investigated; however, the results do not agree as well with those predicted by the Mark VI update to the model. 
This may be due to the use of slightly different (and presumably more accurate) heat transfer and air flow correlations in the Mark VI model. In order to continue using the heat transfer analogy technique it is necessary to modify the $N T U^{*}$ predicted by Equation (4.13). A correction factor of 1.33 was found to yield adequate agreement between the heat transfer analogy technique and the Mark VI model; therefore, when evaluating the heat exchanger effectiveness in Equation (4.15), $N T U^{*}$ is calculated using the correction factor of 1.33

$U^{*}$ is the overall air side conductance with condensation and is calculated using Eq. (4.14).

$$
U^{*}=\frac{\frac{U_{a}}{c_{a}}}{1+\frac{c_{s} U_{a} A_{a}}{c_{a} U_{f} A_{f}}}
$$

where $U_{a}$ and $U_{f}$ are the air side and liquid side conductances, respectively, $A_{f}$ is the liquid side heat exchanger area, and $c_{a}$ is the specific heat of the moist air entering the coil.

For heat exchangers exposed to humid air, the heat transfer analogy method described above is implemented in place of the conventional $\varepsilon-N T U$ technique that was described above. All heat exchanger inputs, such as $U_{a}, A_{a}, U_{f}$, and $A_{f}$, are determined using the DOE/ORNL Heat Pump Design Model operated at its default settings; therefore, these values adequately represent the condenser within a conventional space cooling application. The effectiveness of a cross-flow heat exchanger with condensation and no 
mixing is given by Eq. (4.15).

$$
\varepsilon_{C}=1-\exp \left[\left(\frac{1}{m^{*}}\right)\left(N T U^{*}\right)^{0.22}\left\{\exp \left[-m^{*}\left(N T U^{*}\right)^{0.78}\right]-1\right\}\right]
$$

Equation (4.15) is the same correlation for a cross-flow sensible heat exchanger with $m^{*}$ substituted for the capacitance ratio and $N T U^{*}$ used in place of $N T U$. The actual heat transfer in a heat exchanger with condensation is the product of the maximum heat transfer and the heat exchanger effectiveness. The maximum enthalpy change on the air side occurs when the incoming air is cooled until it is saturated and at the same temperature as the fluid that is entering the heat exchanger, $h_{a, s a t}\left(T_{H X, i n, C}\right)$. The heat transfer for the heat exchanger, $\dot{q}_{h x, C}$ is given by equation (4.16).

$$
\dot{q}_{H X, C}=\varepsilon_{C} \dot{m}_{a}\left(h_{a, i n}-h_{a, s a t}\left(T_{H X, i n, C}\right)\right)
$$

where $h_{a, i n}$ is the enthalpy of the air entering the heat exchanger. Using the heat transfer determined from Eq. (4.16), the outlet enthalpies of the air and water streams can be determined using an energy balance.

$$
\begin{aligned}
& h_{a, o u t}=h_{a, \text { in }}-\frac{\dot{q}_{H X, C}}{\dot{m}_{a}} \\
& h_{H X, \text { out }, C}=h_{H X, \text { in }, C}+\frac{\dot{q}_{H X, C}}{\dot{m}_{f}}
\end{aligned}
$$

Because the effective specific heat of air, $c_{s}$, in Eq. (4.11) is dependent on the outlet temperature of the heat transfer fluid; therefore, this heat exchanger model is not explicit and an iterative solution to the equations is required, as shown by the flow chart shown in Figure 4.8. 
Braun et al. suggest calculating the heat transfer for the heat exchanger configuration assuming that the heat exchanger is completely wet and completely dry; subsequently, it is suggested that the higher heat transfer predicted by the two methods is chosen since both assumptions tend to under-predict the actual heat transfer in the heat exchanger when the coil is partially wet and therefore the higher value will be more accurate. With the completely dry assumption, the latent heat transfer is neglected and the heat transfer is too low. With the completely wet assumption, the model predicts that the air is humidified in the portion of the heat exchanger that is actually dry. The model artificially humidifies the air in the dry section and reduces the total cooling capacity compared to the actual heat exchanger. The assumption that yields higher heat transfer therefore depends on the heat exchanger operating conditions. 


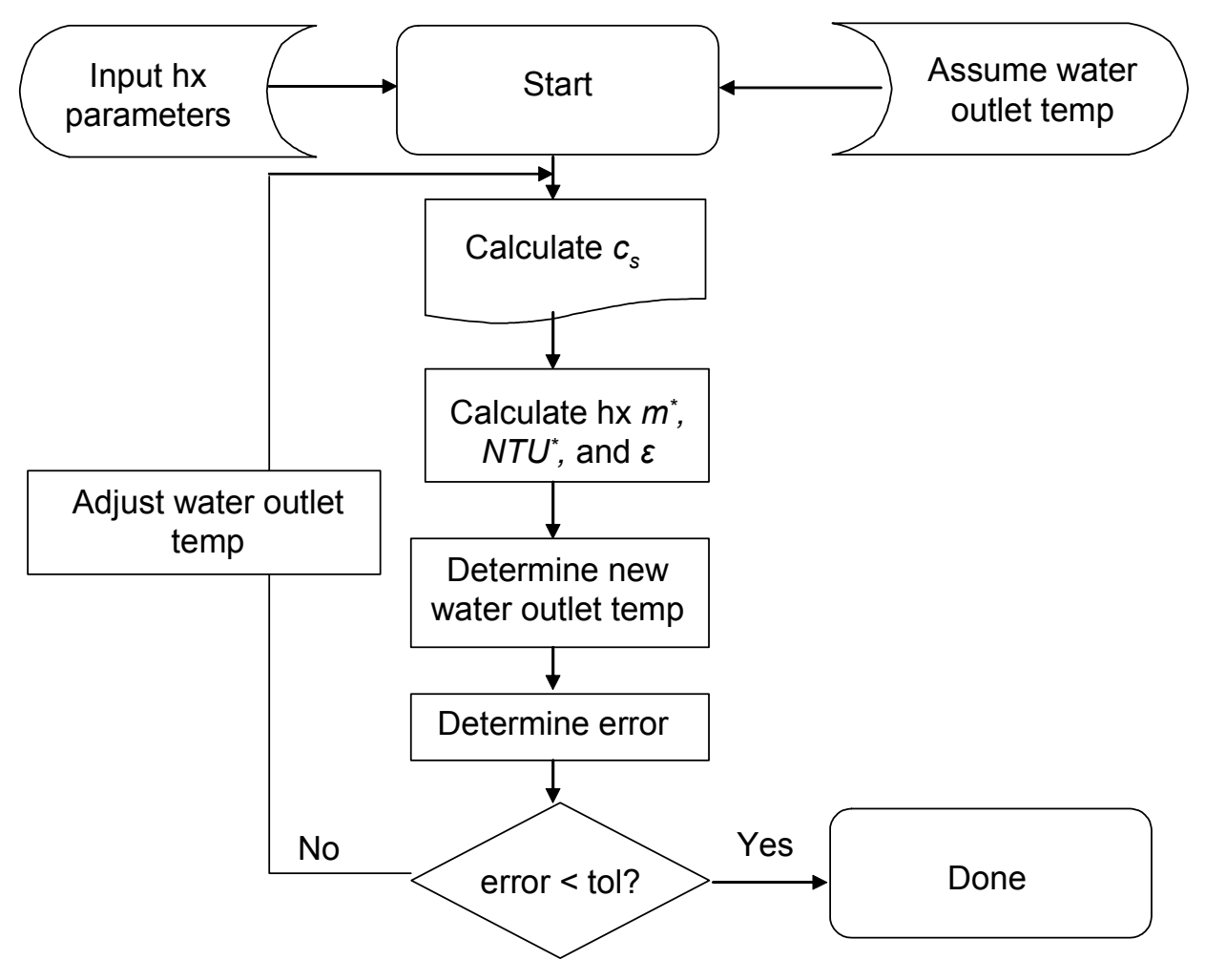

Figure 4.8. Condensing heat exchanger solution flow chart

The rate of heat transfer and therefore the air exit enthalpy is determined based on the equations described above, however the exit relative humidity of the air must still be determined. To determine the outlet air temperature, the air is assumed to flow over a condensed water layer with a constant surface temperature, $T_{s, e f f}$. The process associated with the air flowing over the water surface is similar to a sensible heat exchanger in which a fluid flow is exposed to a constant wall temperature. Using this analogy, the airside number of transfer units, $N T U_{a}$, can be determined for this sensible heat exchanger using the air side conductance and is given by Eq. (4.18).

$$
N T U_{a}=\frac{U_{a} A_{a}}{\dot{m}_{a} c_{a}}
$$

Since the water layer acts as a reservoir with infinite capacity, the effectiveness, $\varepsilon_{a}$, may 
be determined based on a heat exchanger with a thermal capacitance ratio of 0 , given in Eq. (4.19).

$$
\varepsilon_{a}=1-\exp \left(-N T U_{a}\right)
$$

The effective surface enthalpy of the air is determined in Eq. (4.20).

$$
h_{s, \text { eff }}=h_{a, \text { in }}+\frac{h_{a, \text { out }}-h_{a, \text { in }}}{1-\exp \left(-N T U_{a}\right)}
$$

$T_{s, \text { eff }}$ is the temperature associated with saturated moist air with enthalpy, $h_{s, \text { eff. Assuming }}$ constant specific heat for the air stream, Eq. (4.20) is rearranged to yield

$$
T_{a, \text { out }}=T_{s, \text { eff }}+\left(T_{a, \text { in }}-T_{s, \text { eff }}\right) \exp \left(-N T U_{a}\right)
$$

The relative humidity can be determined using $T_{a, \text { out }}$ and $h_{a, \text { out }}$. Using the heat transfer analogy method, the exiting air temperature, relative humidity, and condensation rate can be determined for the heat exchanger.

The results using the method described above can be compared to the results from the DOE/ORNL Heat Pump Design Model Mark VI by using the default air inlet conditions and varying the air volumetric flow rate. The model was run using the default heat exchanger configuration in the DOE/ORNL model and the parameters are given in Table 4.1. At each flow rate the DOE/ORNL model calculates different values for air side conductance, total conductance, and water temperature at the inlet to the cold heat exchanger. The water side conductance may be calculated using the values of total conductance and air side conductance. The DOE/ORNL model uses a cold heat exchanger with a phase change on the refrigerant side that acts like a fluid flow with an 
infinite capacity. In the AMRR, the refrigerant (water) experiences a temperature change as it progresses through the evaporator. In order to compare the performance of the AMRR with the DOE/ORNL results, the water mass flow rate is set to a high value in order to achieve effectively the same infinite capacity rate seen in the refrigerant in the DOE/ORNL model. The two models were then compared over varying volumetric flow rates of air into the heat exchanger and the results are shown in Figure 4.9.

Table 4.1. Heat exchanger model inputs

\begin{tabular}{|c|c|}
\hline Parameter & Value \\
\hline inlet air dry bulb temperature & $80^{\circ} \mathrm{F}$ \\
\hline inlet air wet bulb temperature & $67^{\circ} \mathrm{F}$ \\
\hline inlet water temperature & varies with air flow rate \\
\hline air side conductance & varies with air flow rate \\
\hline total conductance & varies with air flow rate \\
\hline air side area & $223.2 \mathrm{ft}^{2}$ \\
\hline water side area & $14.74 \mathrm{ft}^{2}$ \\
\hline water flow rate & $\begin{array}{r}\text { set to a high value to simulate infinite } \\
\text { water side capacitance }\end{array}$ \\
\hline
\end{tabular}




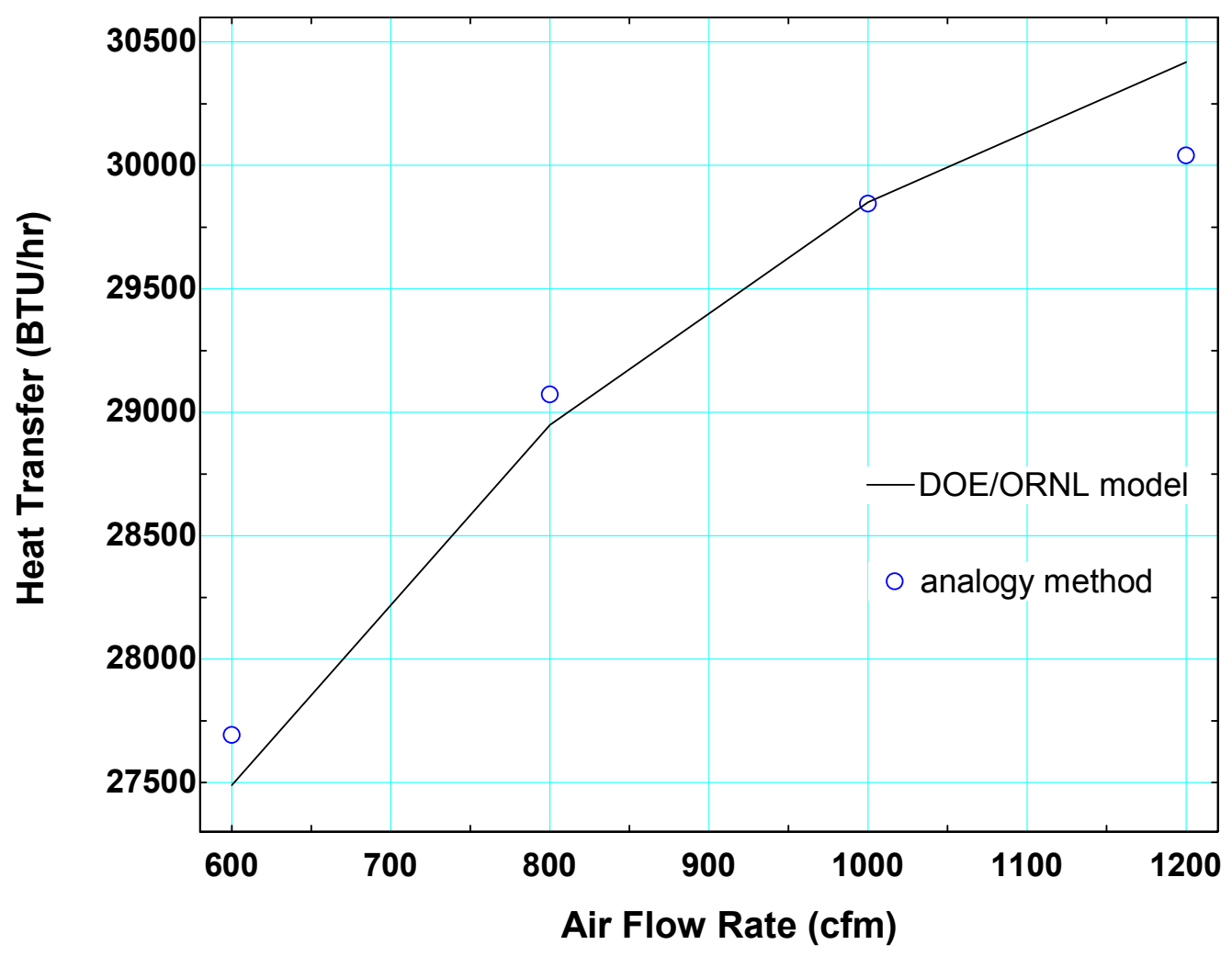

Figure 4.9. Heat transfer rate as a function of the air flow rate predicted by the DOE/ORNL Mark VI Model and the heat transfer analogy method

Figure 4.9 indicates that the analogy method using the correction factor described from Equation (4.13) predicts a heat transfer rate that agrees well with existing and accepted models of condensing heat exchangers over a range of inlet airflow rates. The maximum observed discrepancy between the DOE/ORNL model and the analogy method is less than $1.5 \%$.

The results of the outlet air relative humidity from the analogy method were also compared to the DOE/ORNL model. The parameter chosen to compare the results is the sensible heat ratio, which is defined as the ratio of sensible heat transfer divided by the 
total heat transfer. The sensible heat ratio from the DOE/ORNL model and the analogy method are shown in Figure 4.10 for varying air flow rates.

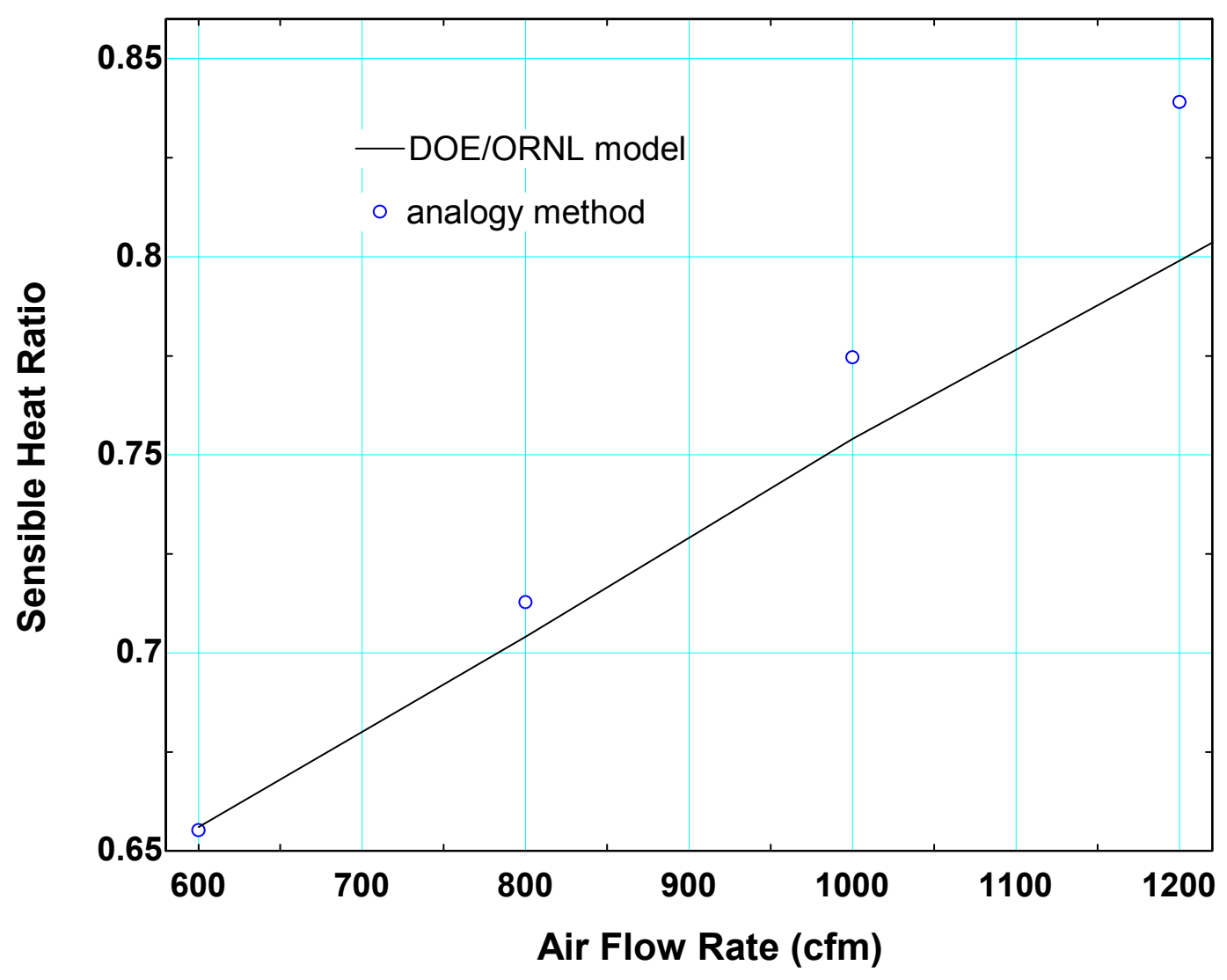

Figure 4.10. Sensible heat ratio as a function of the air flow rate predicted by the DOE/ORNL Mark VI Model and the heat transfer analogy method

The maximum error between the analogy method and DOE/ORNL model is less than 5\% for the flow rates shown in Figure 4.10. Inaccurate predictions of the sensible heat ratio will cause errors in condensation rate predictions. The discrepancies between the analogy method and the DOE/ORNL model are acceptable. This method has been shown 
to predict the total heat transfer in the heat exchanger more accurately than water condensation rates. Therefore, this model is an effective way to determine heat transfer in a condensing coil using a computationally simple method while obtaining accurate results.

\subsubsection{Results for Space Conditioning}

It is useful to directly compare the predicted performance of an AMR to a standard vapor compression system. To accomplish this, the DOE/ORNL Heat Pump Design Model developed and available online was exercised using its default conditions. The parameters used to carry out the comparison are summarized in Table 4.2. The AMR bed model coupled to the heat exchanger models described above were operated in order to determine how system performance varies with the regenerator volume, aspect ratio $(L / d)$, and bed layering.

Table 4.2. Parameters used for space cooling comparative study.

\begin{tabular}{|l|c|l|c|}
\hline \multicolumn{1}{|c|}{ Parameter } & Value & \multicolumn{1}{c|}{ Parameter } & Value \\
\hline heat rejection temp. & $308.2 \mathrm{~K}$ & hot heat exchanger UA & $1430 \mathrm{~W} / \mathrm{K}$ \\
\hline load temperature & $299.8 \mathrm{~K}$ & motor efficiency & 0.9 \\
\hline cooling capacity & $8.76 \mathrm{~kW}$ & pump efficiency & 0.7 \\
\hline maximum applied field & $1.5 \mathrm{Tesla}$ & heat transfer fluid & Water \\
\hline cold air mass flow rate & $0.57 \mathrm{~kg} / \mathrm{s}$ & number of beds & 6 \\
\hline hot air mass flow rate & $1.42 \mathrm{~kg} / \mathrm{s}$ & period & $0.2 \mathrm{sec}(5 \mathrm{~Hz})$ \\
\hline cold heat exchanger UA & $877.3 \mathrm{~W} / \mathrm{K}$ & sphere size for packing & $0.2 \mathrm{~mm}$ \\
\hline
\end{tabular}

The DOE/ORNL Heat Pump Design Model predicts a COP of 3.10 for the baseline vapor compression cycle, including $0.59 \mathrm{~kW}$ of fan power. The AMR model was subsequently 
run at the same conditions and using the same heat exchanger sizes (as indicated by the $U A$ values in Table 4.2) and air flow rates. The numerical model does not account for fan power; therefore the fan power predicted by the Heat Pump Design Model was added to the total power predicted by the numerical model and used to correct the predicted COP for the AMR cycle.

Figure 4.11 illustrates the predicted COP as a function of regenerator volume for a layered and non-layered bed; these curves were generated using a refrigeration capacity of $8.76 \mathrm{~kW}$ and the optimal aspect ratio for each volume. Figure 4.11 indicates that an AMR cycle may be capable of achieving higher values of COP than an equivalent vapor compression cycle; however, the COP that can be achieved depends strongly on the volume of the magnetic regenerator bed used. As the AMR regenerator volume increases, the operating efficiency increases. The layered bed significantly out-performs the non-layered bed for any volume. 


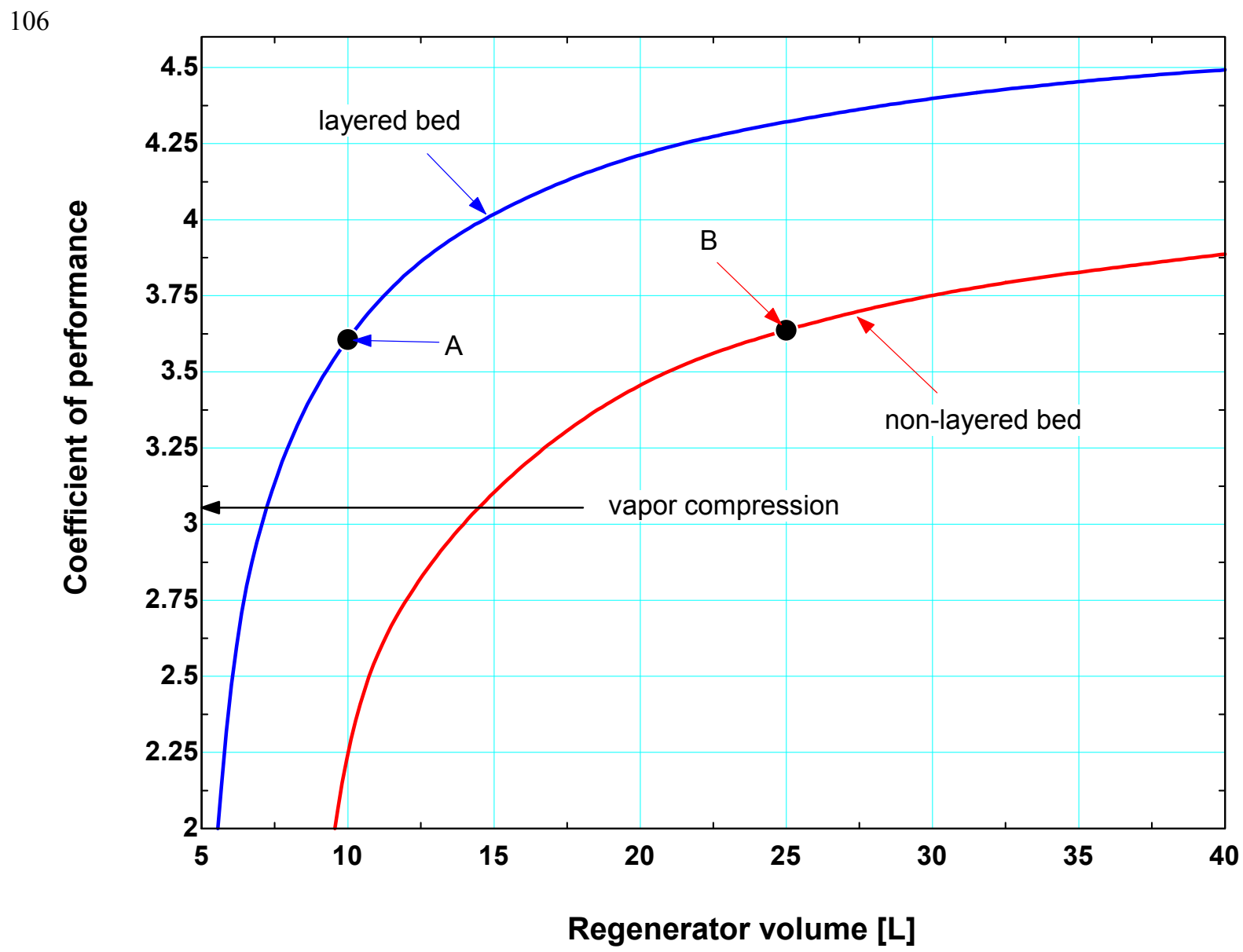

Figure 4.11. COP of a layered and non-layered bed producing $8.76 \mathrm{~kW}$ of refrigeration at its optimal aspect ratio. Also shown is the COP for a comparable vapor compression cycle.

The sensitivity of the COP of the AMR cycle to the heat rejection temperature was investigated by carrying out a parametric study using a particular regenerator design (volume and aspect ratio) for a layered and a non-layered bed. The regenerator designs selected for this parametric analysis were selected such that the COP was higher than the equivalent vapor compression system at the design condition and also so that the COP of the layered and non-layered regenerators were approximately equal. For this study, a 10 
$\mathrm{L}$ layered bed and a $25 \mathrm{~L}$ non-layered, both operating at their optimum aspect ratios were chosen; these are shown in Figure 4.11 as designs A and B, respectively. The regenerator bed material, and therefore the Curie temperature distribution in the layered regenerator bed, was held constant at the values used to develop Figure 4.11. The heat rejection temperature (the temperature of the air entering the hot heat exchanger) was varied while the cooling capacity was held constant at $8.76 \mathrm{~kW}$. The constant cooling load was achieved under the varying operating conditions by selecting the appropriate mass flow rate. The results are shown in Figure 4.12. 


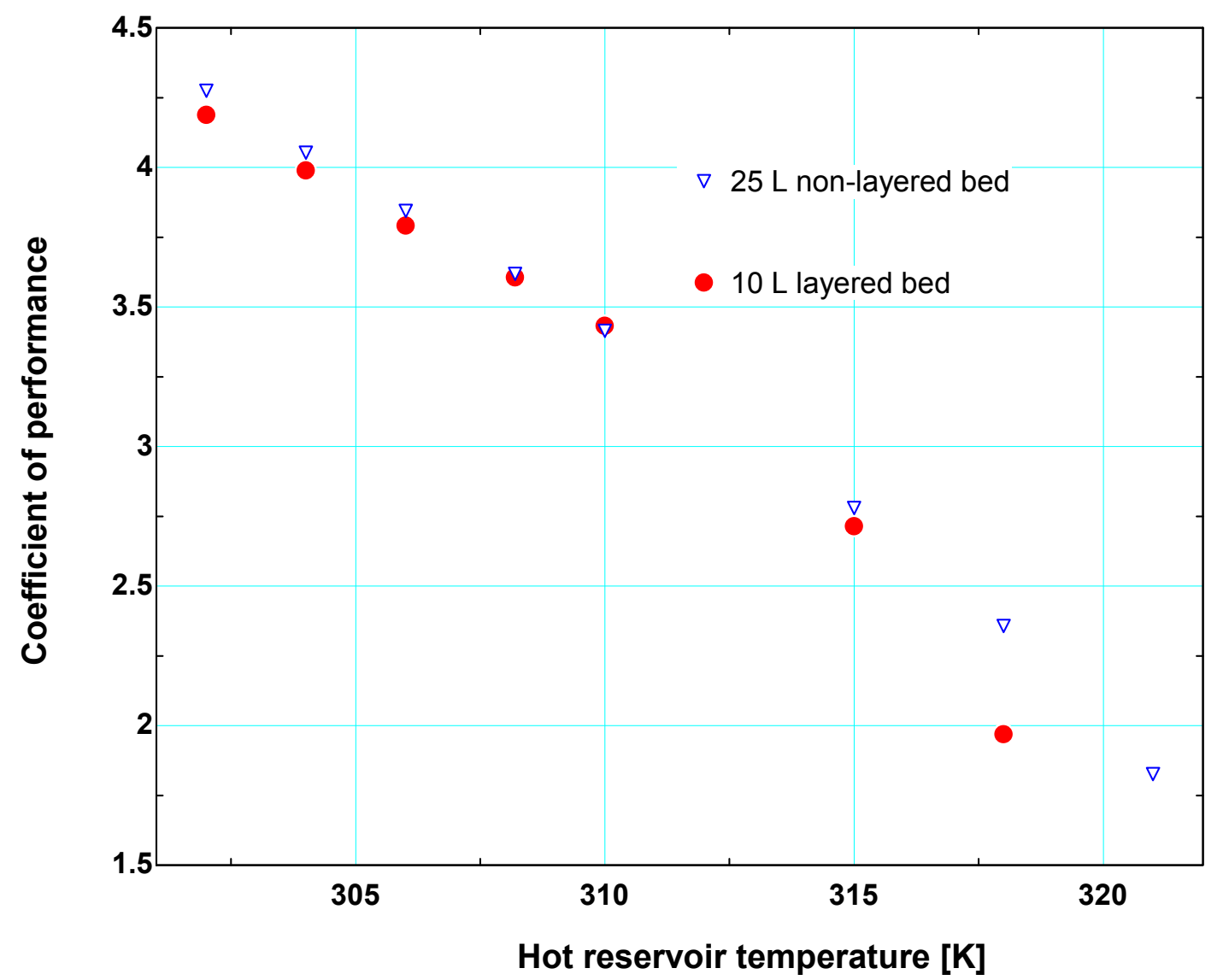

Figure 4.12. $\mathrm{COP}$ of a $10 \mathrm{~L}$ layered and $25 \mathrm{~L}$ non-layered bed producing $8.76 \mathrm{~kW}$ of refrigeration at their optimal aspect ratios as a function of heat rejection temperature.

In Figure 4.12, the layered bed is no longer capable of producing $8.76 \mathrm{~kW}$ when the heat rejection temperature rises above $318 \mathrm{~K}\left(113^{\circ} \mathrm{F}\right)$ and the non-layered bed is unable to produce the desired cooling when the heat rejection temperature is above $321 \mathrm{~K}\left(118^{\circ} \mathrm{F}\right)$. The non-layered bed operates at a slightly higher coefficient of performance than the layered bed as the heat rejection temperature deviates from the design temperature of 308.2 K although their volumes are considerably different. 


\subsection{Refrigeration Application}

\subsubsection{Heat Exchanger Modeling}

The hot and cold heat exchangers, the equivalent of the condenser and evaporator, for the refrigeration application are modeled as cross flow air-to-liquid sensible heat exchangers. The same method is used to model the hot and cold heat exchangers for the refrigeration application as was previously described for the hot heat exchanger in the space cooling application. Condensation and frost accumulation are not considered when modeling the cold heat exchanger. The heat exchanger configuration for the refrigeration application is identical to the space cooling application as shown in Figure 4.3. Therefore, Equations (4.4) - (4.10) apply to the hot heat exchanger and analogous equations are used to calculate the cold heat exchanger performance. The same process illustrated in Figure 4.4 is used to obtain a system performance solution.

\subsubsection{Results for Refrigeration Application}

The model was used to predict the performance of an AMRR operating at freezer temperatures. Gan (1998) studied the total conductance of heat exchangers in a residential $500 \mathrm{BTU} / \mathrm{hr}$ refrigerator/freezer with a split freezer and refrigerator cycle. The conductance of the AMRR hot heat exchanger conductance is taken to be the sum of the conductances of the refrigerator and freezer condensers, and the cold heat exchanger conductance is the sum of the conductances of the refrigerator and freezer evaporators.

The resulting heat exchanger parameters and other model inputs used to carry out the analysis are summarized in Table 4.3. 
Table 4.3. Parameters used for refrigeration analysis.

\begin{tabular}{|l|c|l|c|}
\hline \multicolumn{1}{|c|}{ Parameter } & Value & \multicolumn{1}{c|}{ Parameter } & Value \\
\hline heat rejection temp. & $305.4 \mathrm{~K}$ & hot heat exchanger UA & $72.8 \mathrm{~W} / \mathrm{K}$ \\
\hline load temperature & $255.4 \mathrm{~K}$ & motor efficiency & 0.9 \\
\hline cooling capacity & $150 \mathrm{~W}$ & pump efficiency & 0.7 \\
\hline maximum applied field & $1.5 \mathrm{Tesla}$ & heat transfer fluid & $\begin{array}{c}50 \% \text { ethylene glycol, } \\
50 \% \text { water }\end{array}$ \\
\hline cold air mass flow rate & $0.0607 \mathrm{~kg} / \mathrm{s}$ & number of beds & 6 \\
\hline hot air mass flow rate & $0.1091 \mathrm{~kg} / \mathrm{s}$ & period & $0.2 \mathrm{sec}(5 \mathrm{~Hz})$ \\
\hline cold heat exchanger UA & $85.99 \mathrm{~W} / \mathrm{K}$ & sphere size for packing & $0.2 \mathrm{~mm}$ \\
\hline
\end{tabular}

Figure 4.13 illustrates the predicted COP as a function of regenerator volume for a layered and non-layered bed; these curves were generated using a refrigeration capacity of $150 \mathrm{~W}$ and the optimal aspect ratio for each volume, and fan power is ignored. From Jaehnig (1999), a typical vapor compression system operating under these condition has a COP of 1.9. Comparing Figure 4.13 to Figure 4.11 shows that layering the regenerator bed of an AMR refrigerator has a more significant impact on performance than layering a space cooling AMR. This behavior results because as the hot and cold reservoir temperature span increases, the regenerator material of a non-layered bed operates further from the temperature corresponding to its maximum magnetocaloric point (Curie temperature) on average. The material in a layered bed is chosen such that the local temperature is near the Curie temperature of the material at that regenerator location. For the refrigerator application, the non-layered bed is unable to achieve a COP that is competitive with current vapor compression technology, while the layered bed is able to exceed vapor compression performance with an adequately large regenerator. As with 
the space cooling application, as the AMR regenerator volume increases the operating efficiency also increases and the layered bed out-performs the non-layered bed.

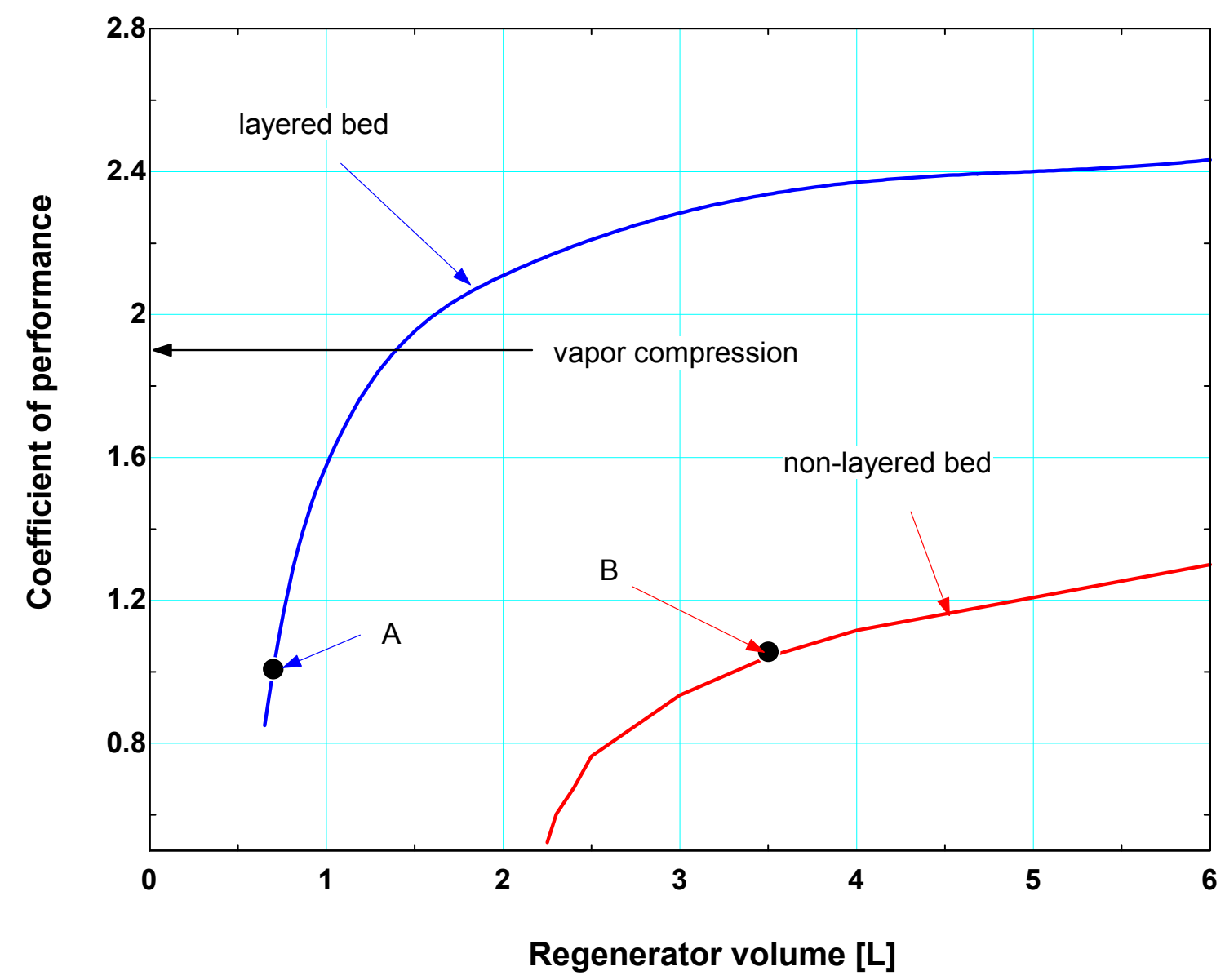

Figure 4.13. $\mathrm{COP}$ of a layered and non-layered bed producing $150 \mathrm{~W}$ of refrigeration at its optimal aspect ratio. Also shown is the COP for a comparable vapor compression cycle.

The sensitivity of the COP of the AMR refrigeration cycle to the load temperature was investigated by carrying out a parametric study using a particular regenerator design 
(volume and aspect ratio) for a layered and a non-layered bed. A moderate refrigerator COP was chosen from the data presented in Figure 4.13 and the volume of the layered and non-layered regenerators were chosen such that the COP of each system was approximately equal. For this study, a $0.7 \mathrm{~L}$ layered bed and a $3.5 \mathrm{~L}$ non-layered both operating at their optimum aspect ratios were chosen; these are shown in Figure 4.13 as designs $\mathrm{A}$ and $\mathrm{B}$, respectively. The regenerator bed material, and therefore the Curie temperature distribution in the layered regenerator bed, was held constant. The load temperature (the temperature of the air entering the cold heat exchanger) was varied between $255 \mathrm{~K}\left(0^{\circ} \mathrm{F}\right)$ and $267 \mathrm{~K}\left(20^{\circ} \mathrm{F}\right)$ while the cooling capacity was held constant at $150 \mathrm{~W}$. The constant cooling load was achieved under the varying operating conditions by selecting the appropriate mass flow rate. The AMR beds selected for this study were not able to produce $150 \mathrm{~W}$ at lower load temperatures; thus only systems operating above the load temperatures used to generate Figure 4.13 were constrained to a $150 \mathrm{~W}$ cooling capacity. The results are shown in Figure 4.14. 


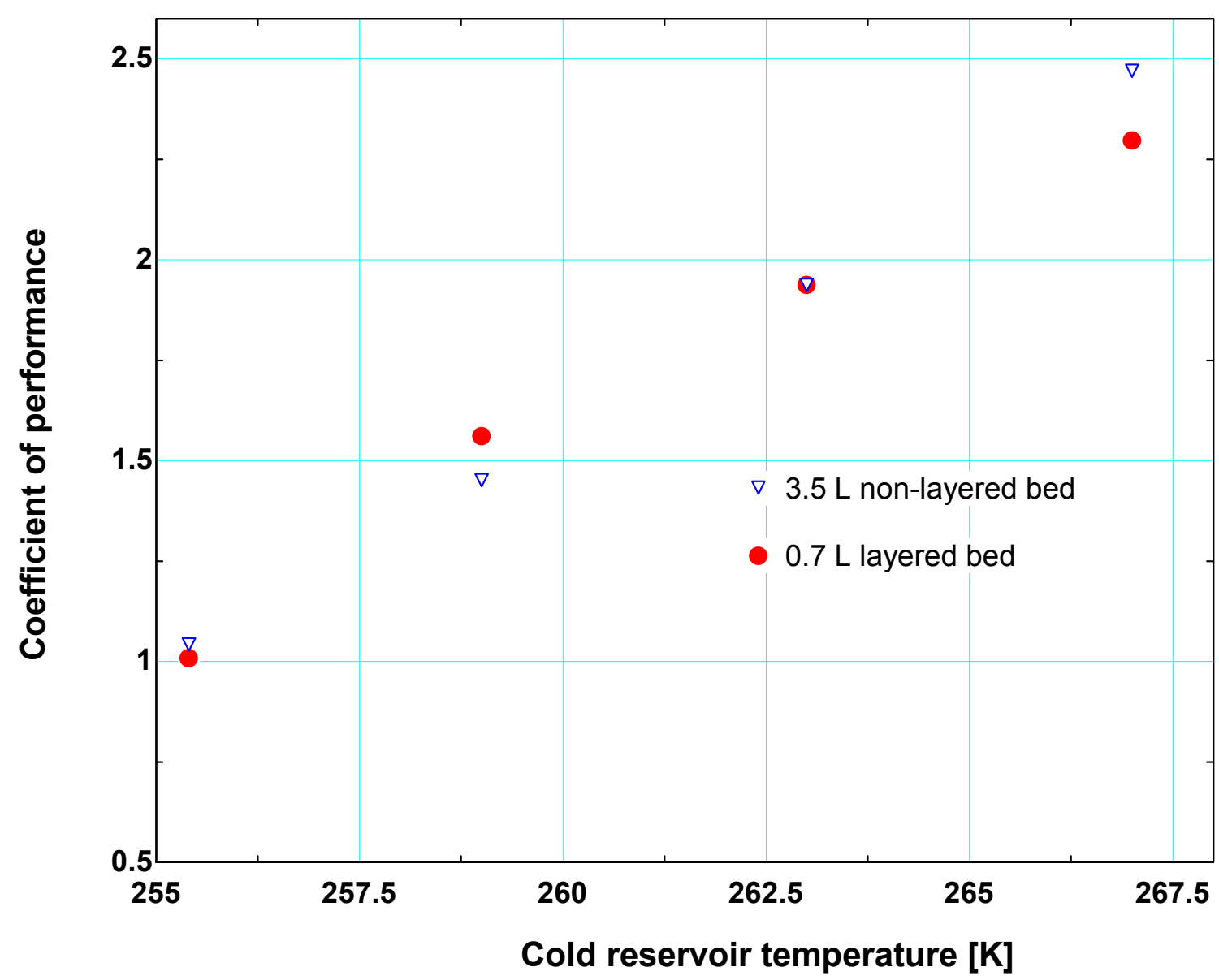

113

Figure 4.14. $\mathrm{COP}$ of a $0.7 \mathrm{~L}$ layered and $3.5 \mathrm{~L}$ non-layered bed producing $150 \mathrm{~W}$ of refrigeration at their optimal aspect ratios as a function of load temperature.

For temperatures below the load temperature used to generate Figure 4.13, the calculated mass flow rate required to produce $150 \mathrm{~W}$ the original load temperature was held constant while the load temperature was varied between $255 \mathrm{~K}\left(0^{\circ} \mathrm{F}\right)$ and $244 \mathrm{~K}\left(-20^{\circ} \mathrm{F}\right)$. At each load temperature a new cooling capacity and $C O P$ were calculated and the results are shown in Figure 4.15 and 4.16. 
114

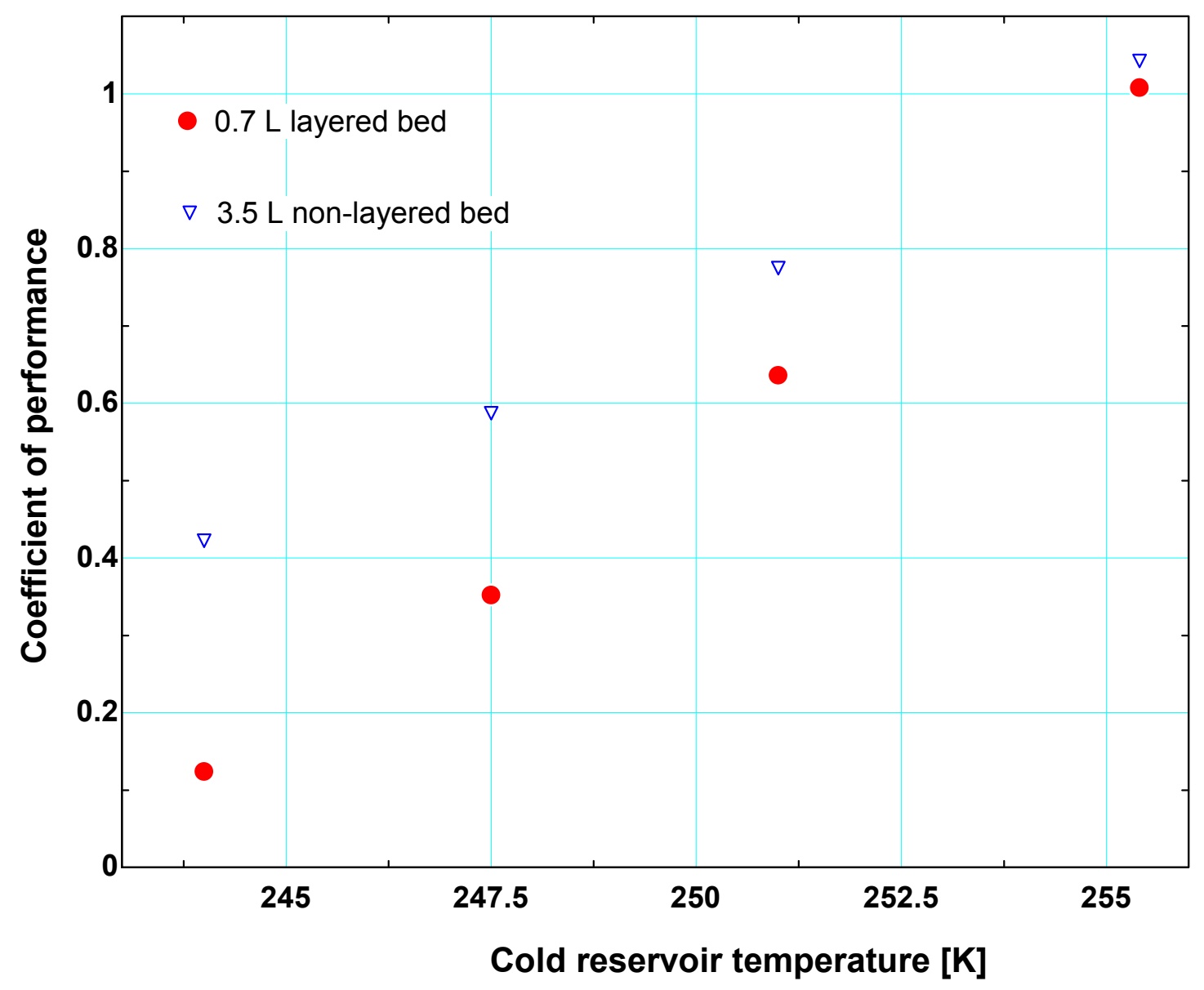

Figure 4.15. COP of a $0.7 \mathrm{~L}$ layered and $3.5 \mathrm{~L}$ non-layered bed operating at their optimal aspect ratios and with constant mass flow rates as a function of load temperature. 


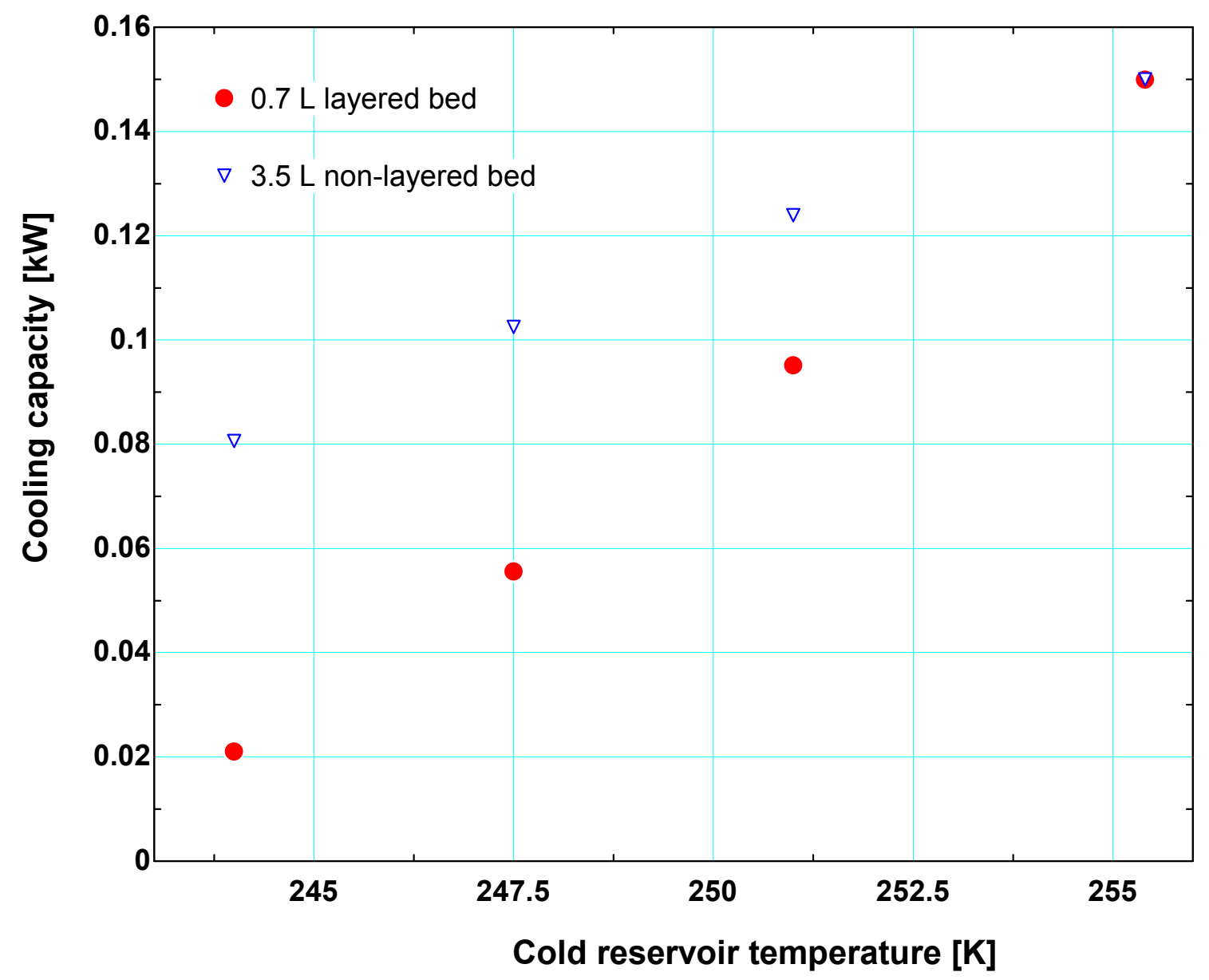

Figure 4.16. Cooling capacity of a $0.7 \mathrm{~L}$ layered and $3.5 \mathrm{~L}$ non-layered bed operating at their optimal aspect ratios and with constant mass flow rates as a function of load temperature.

In Figures 4.15 and 4.16, the non-layered bed produces a larger cooling load at a higher COP than the layered bed as the load temperature decreases. These figures also show that the efficiency and cooling capacity of an AMRR used for a refrigerator are very sensitive to the load temperature. 


\section{Chapter $5 \quad$ Parametric Studies}

\subsection{Advanced Regenerator Results}

The results presented in the previous chapters were entirely related to a specific regenerator geometry (200 $\mu \mathrm{m}$ spheres) and material (an Erbium/Gadolinium alloy). This configuration represents a relatively near term technology; the Erbium/Gadolinium alloy has been fabricated and tested and can be made in spherical form. However, in the long term there are more advanced regenerator geometries and materials that may become available. It is important to quantify the potential of these more advanced configurations in order to assess the long-term promise of magnetic refrigeration technology. In this section, an advanced AMRR geometry with a high-performance magnetocaloric material is simulated.

The advanced geometry suggested by Astronautics consists of a parallel plate regenerator with flow channels that are $50 \mu \mathrm{m}$ wide; the distance between the channels is adjusted to achieve a specific porosity. The plates are assumed to be aligned such that the flow channel is uninterrupted in the flow direction, resulting in a configuration with high heat transfer coefficients and low pressure drop. In this section the flow in the channels is modeled as being fully developed over the entire length of the regenerator. The regenerator material is assumed to be a layered bed of $\mathrm{La}\left(\mathrm{Fe}_{\mathrm{x}} \mathrm{Si}_{1-\mathrm{x}}\right)_{13} \mathrm{H}_{\mathrm{y}}$ compound with properties consistent with those described by Fujita et al. (2003).

\subsubsection{Advanced Regenerator Bed Correlations and Material Properties}

The friction factor for flow between two infinite plates $\left(f_{f}\right)$ is given by Kays and London (1964) 


$$
f_{f}=\frac{24}{R e_{f}}
$$

and the Nusselt number in the fluid $\left(N u_{f}\right)$ for constant heat flux on each side is a constant value of 8.235. The effective static conduction through the regenerator material and fluid $\left(k_{\text {static }}\right)$ is calculated by treating the thermal resistances of the fluid and solid in parallel.

$$
k_{\text {static }}=k_{f} \varepsilon+k_{r}(1-\varepsilon)
$$

where $k_{f}$ is the conductivity of the fluid and $k_{r}$ is the conductivity of the regenerator material, which is approximately a constant $10.4 \mathrm{~W} / \mathrm{m}-\mathrm{K}$ at space conditioning temperatures according to Fujieda et al. (2004) for the Lanthanum alloy. The axial dispersion for a fluid flowing between two infinite plates is given by Beard (2001).

$$
D^{d}=1+\frac{P e_{f}^{2}}{210}
$$

The total effective conductivity, $k_{\text {eff }}$, of the bed can then be calculated according to:

$$
k_{e f f}=k_{\text {static }}+k_{f} D^{d}
$$

As with a packed sphere regenerator, the Biot number for a parallel plate regenerator is not generally below 0.1 , and therefore the magnetic material in the regenerator cannot be considered spatially uniform in temperature. This effect is accounted for by determining the difference between the volumetric mean temperature of the wall between flow channels and its surface temperature and using this result to modify the heat transfer coefficient, as described in Section 2.6 for a sphere. After following through the derivation in Cartesian rather than spherical coordinates, the result becomes: 


$$
h_{e f f}=\frac{h}{1+\frac{B i}{3}}
$$

where $h_{\text {eff }}$ is the heat transfer coefficient considering internal temperature gradients in the regenerator matrix. The Biot number for this case is defined as

$$
B i=\frac{h \cdot b}{2 k_{r}}
$$

where $b$ is the wall thickness between flow passages.

A sufficiently resolved set of material property data for $\mathrm{La}\left(\mathrm{Fe}_{\mathrm{x}} \mathrm{Si}_{1-\mathrm{x}}\right){ }_{13} \mathrm{H}_{\mathrm{y}}$ was not available for this study; a relatively coarse set of data was presented in the paper by Fujita et al. (2003). Therefore, the thermodynamic properties of these compounds were simulated by using the existing GdEr data and multiplying it by a constant scaling factor. Based on figures presented by Fujita et al. (2003), a scaling factor of 2.0 was chosen for the specific heat at constant magnetic field and the partial differential of entropy with respect to magnetic field. This multiplier reflects the enhanced magnetocaloric effect of the advanced material. Figure 5.1 shows material properties given by Fujita et al. and Figure 5.2 shows material properties generated using a scaling factor for GdEr data. 


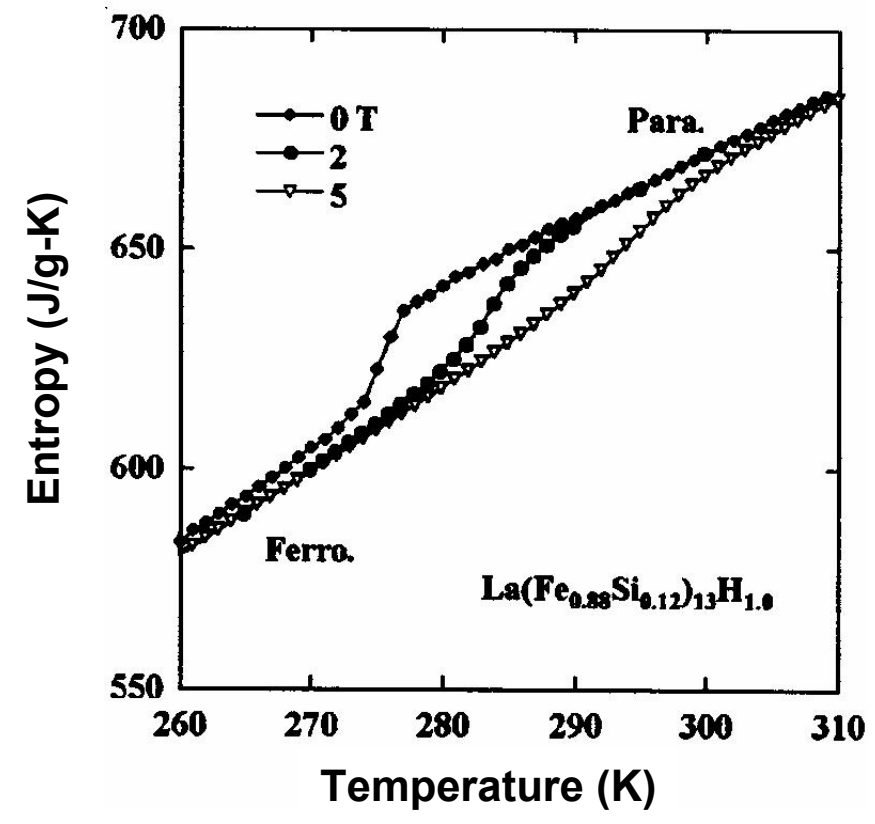

Figure 5.1. Entropy as a function of temperature for $\mathrm{La}\left(\mathrm{Fe}_{\mathrm{x}} \mathrm{Si}_{1-\mathrm{x}}\right)_{13} \mathrm{H}_{\mathrm{y}}$ from Fujita et al.(2003) 


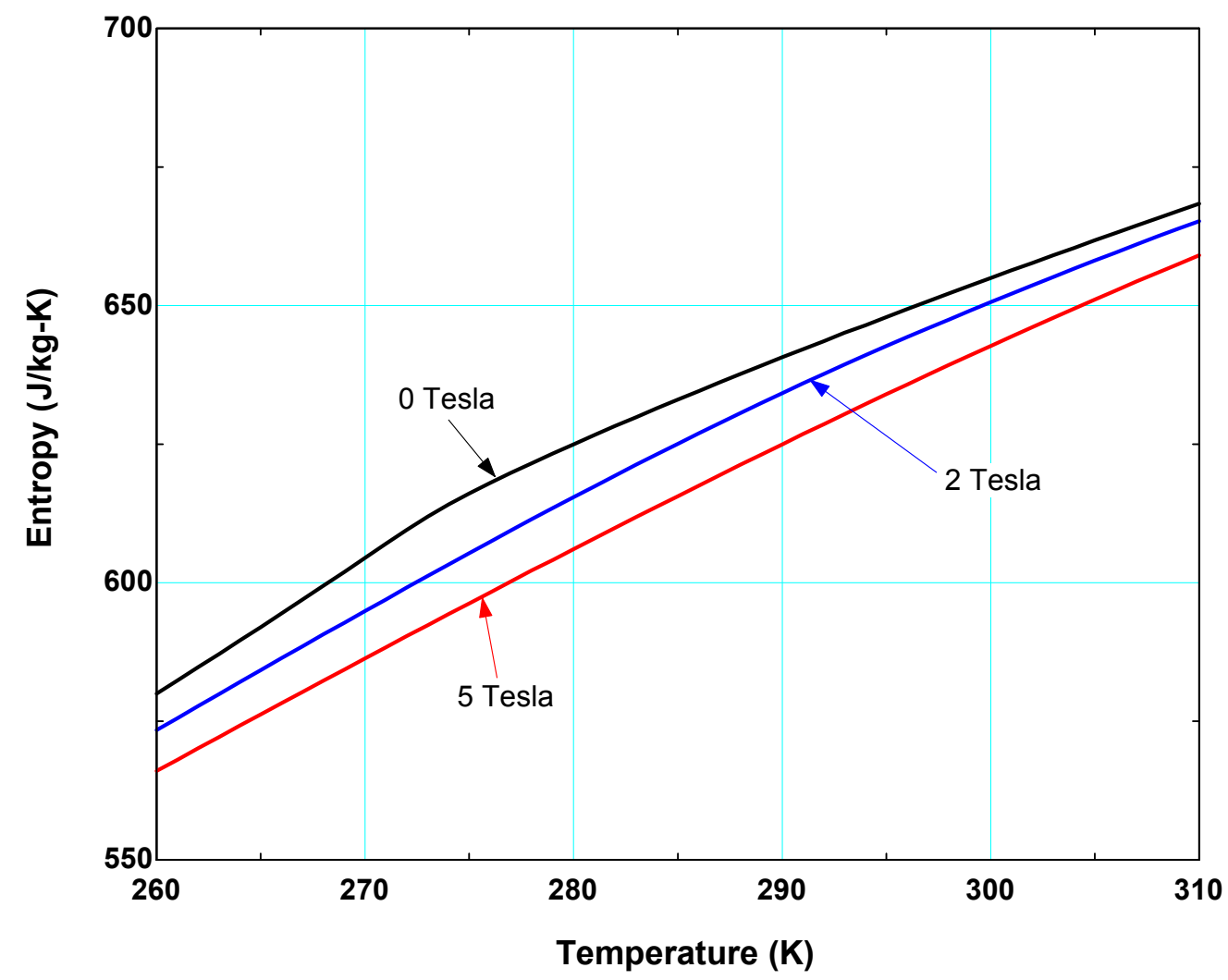

Figure 5.2. Predicted entropy as a function of temperature for advanced material using GdEr properties with a scaling factor.

Note that the increased magnitude of the magnetocaloric effect is captured but the sharp discontinuities in the lines of constant applied field are not reproduced. Nevertheless, we feel that this approach will provide an adequate indication of the potential of this material in the context of an AMRR.

\subsubsection{Optimum Porosity for the Advanced Regenerator}

The effect of the porosity of the advanced regenerator was determined by varying the porosity (i.e., the distance between adjacent channels) while holding all other system parameters constant; the optimal porosity was the value that yielded the highest 
coefficient of performance for a desired cooling capacity. It is assumed that the optimum regenerator porosity is approximately independent of other system parameters and therefore the porosity is not re-optimized during the system studies presented in the subsequent section. The model inputs for the porosity optimization are given in Table 5.1 .

Table 5.1. Parameters used for porosity optimization.

\begin{tabular}{|l|l|l|l|}
\hline Parameter & Value & Parameter & Value \\
\hline heat rejection temp. & $308.2 \mathrm{~K}$ & hot heat exchanger UA & $1430 \mathrm{~W} / \mathrm{K}$ \\
\hline load temperature & $299.8 \mathrm{~K}$ & motor efficiency & 0.9 \\
\hline cooling capacity & $8.76 \mathrm{~kW}$ & pump efficiency & 0.7 \\
\hline maximum applied field & $1.5 \mathrm{Tesla}$ & heat transfer fluid & Water \\
\hline cold air mass flow rate & $0.57 \mathrm{~kg} / \mathrm{s}$ & number of beds & 6 \\
\hline hot air mass flow rate & $1.42 \mathrm{~kg} / \mathrm{s}$ & period & $0.07 \mathrm{sec}(14.2 \mathrm{~Hz})$ \\
\hline cold heat exchanger UA & $877.3 \mathrm{~W} / \mathrm{K}$ & width of flow passage & $0.05 \mathrm{~mm}$ \\
\hline aspect ratio $(L / D)$ & 0.25 & bed layering & linear layering \\
\hline regenerator volume & $2 \mathrm{~L}$ & magnetic material & $\mathrm{La}\left(\mathrm{Fe}_{\mathrm{x}} \mathrm{Si}_{1-\mathrm{x}}\right)_{13} \mathrm{H}_{\mathrm{y}}$ \\
\hline
\end{tabular}

Figure 5.3 shows the effect of porosity on the efficiency of an AMRR producing $8.76 \mathrm{~kW}$ of cooling for a space cooling application. The figure shows that a porosity near 0.55 yields a maximum coefficient of performance under the conditions considered for this simulation. For all subsequent simulations of the advanced regenerator performance, the optimum porosity is assumed to be 0.55 . 


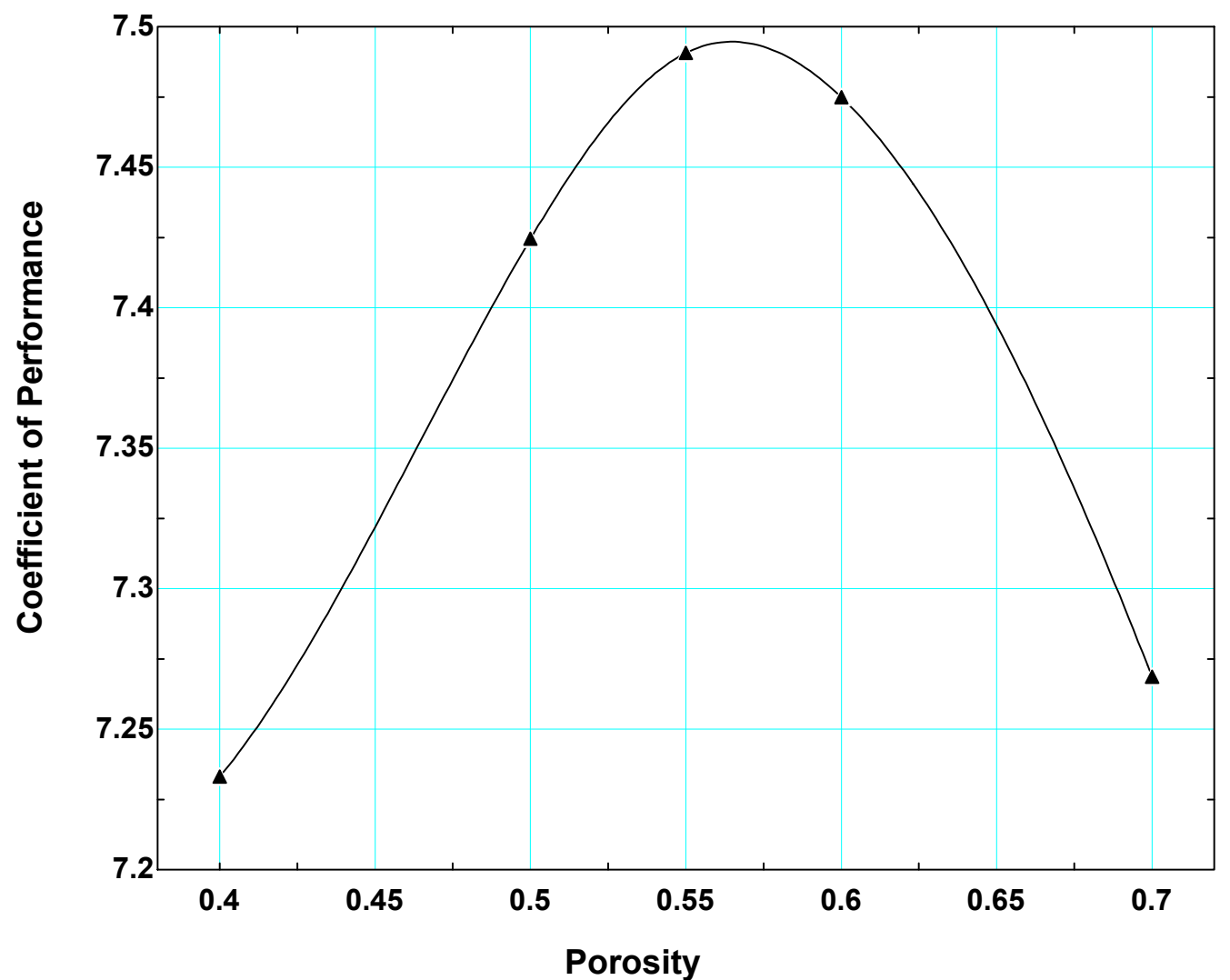

Figure 5.3. COP of a layered advanced regenerator producing $8.76 \mathrm{~kW}$ of refrigeration versus regenerator porosity. Note that an optimal porosity of 0.55 indicates that the channels and the material between the channels will be of nominally equal width.

\subsubsection{Advanced Regenerator Results}

The system studies for the advanced regenerator are carried out in the same way as the original regenerator configuration described in section 4.4.2; the results of the advanced regenerator AMRR simulations are compared to the predicted performance of a standard vapor compression system using the DOE/ORNL Heat Pump Design Model. The parameters used to carry out the comparison are given in Table 5.1 with the exception that the regenerator volume and aspect ratio are now design parameters. Therefore, the AMR bed model coupled to the heat exchanger models were exercised in order to 
determine how system performance varies with the regenerator volume and aspect ratio $(L / d)$

The DOE/ORNL Heat Pump Design Model predicts a COP of 3.10 for the baseline vapor compression cycle, including $0.59 \mathrm{~kW}$ of fan power. The AMR model was subsequently run at the same conditions and using the same heat exchanger sizes (as indicated by the $U A$ values in Table 5.1) and air flow rates. As in section 4.4.2, the fan power predicted by the Heat Pump Design Model was added to the total power predicted by the numerical model and used to correct the predicted COP for the AMR cycle.

Figure 5.4 illustrates the predicted $\mathrm{COP}$ as a function of regenerator volume for a layered regenerator with advanced geometry and materials; these curves were generated using a refrigeration capacity of $8.76 \mathrm{~kW}$ and the optimal aspect ratio for each volume. Figure 5.4 indicates that the AMR cycle with the advanced regenerator geometry and material is capable of producing the same cooling power as a regenerator comprised of packed GdEr spheres but at significantly lower regenerator volumes. A comparison of the performance of the packed sphere regenerators and the advanced regenerator as a function of volume is shown in Figure 5.5. The packed sphere regenerator with a layered bed is able to produce $8.76 \mathrm{~kW}$ of cooling power with a COP comparable to a vapor compression system using a regenerator volume of $5.5 \mathrm{~L}$ whereas the advanced regenerator is capable of producing the same cooling load using a regenerator volume of $0.65 \mathrm{~L}$ and operates at a higher COP. The advanced materials result in an order of magnitude improvement in necessary regenerator volume and a significant increase in performance, which clearly 
shows both the potential of the technology and the need for improved materials and geometries. The enhanced performance of the advanced regenerator is due to the higher magnetocaloric effect exhibited by the magnetic material, the higher cycle frequency used by the AMR, and the more efficient regenerator geometry.

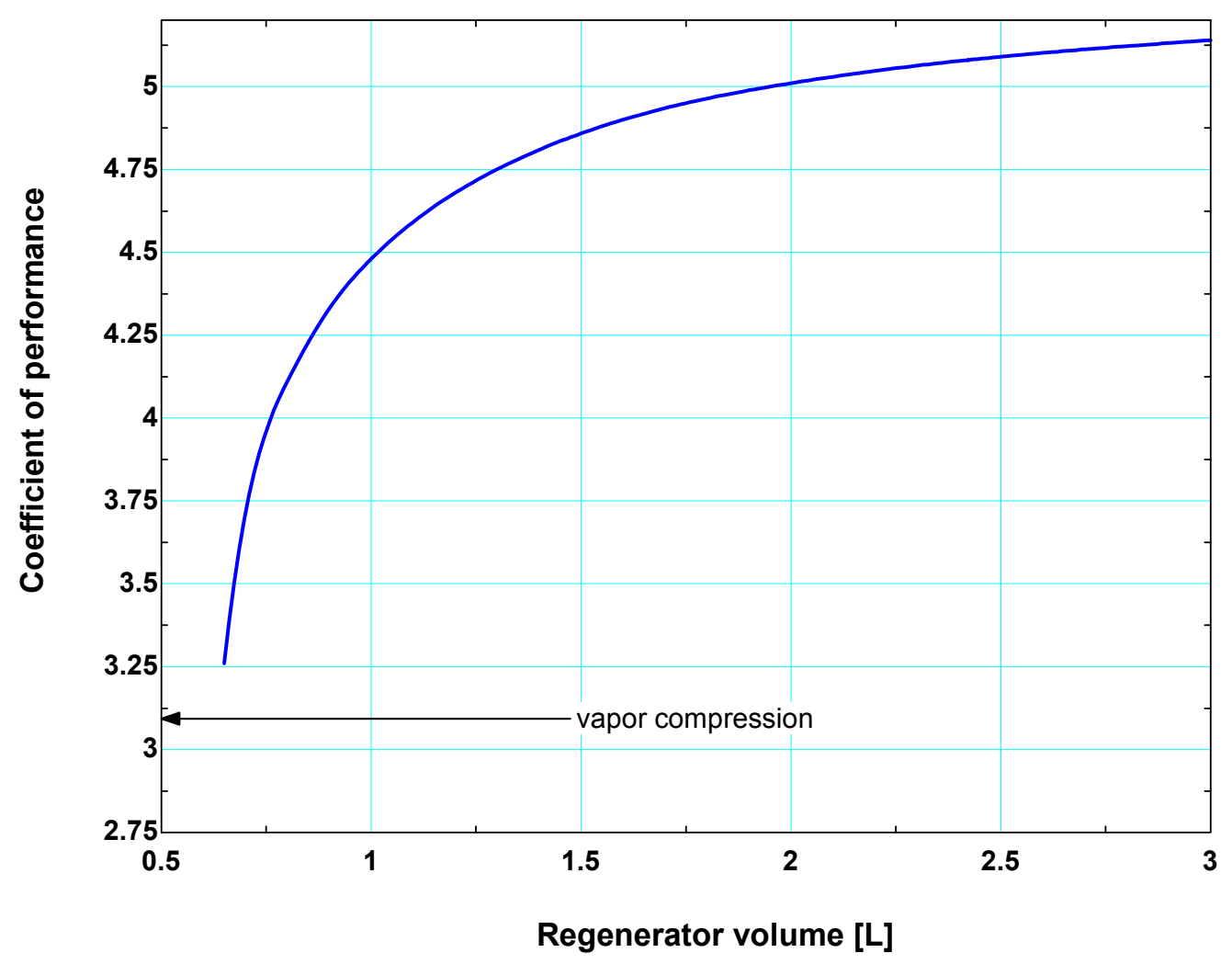

Figure 5.4. COP of an advanced regenerator capable of producing 8.76 $\mathrm{kW}$ of refrigeration at its optimal aspect ratio at conditions given in Table Table 5.1 with a porosity of 0.55. Also shown is the COP for a comparable vapor compression cycle. 


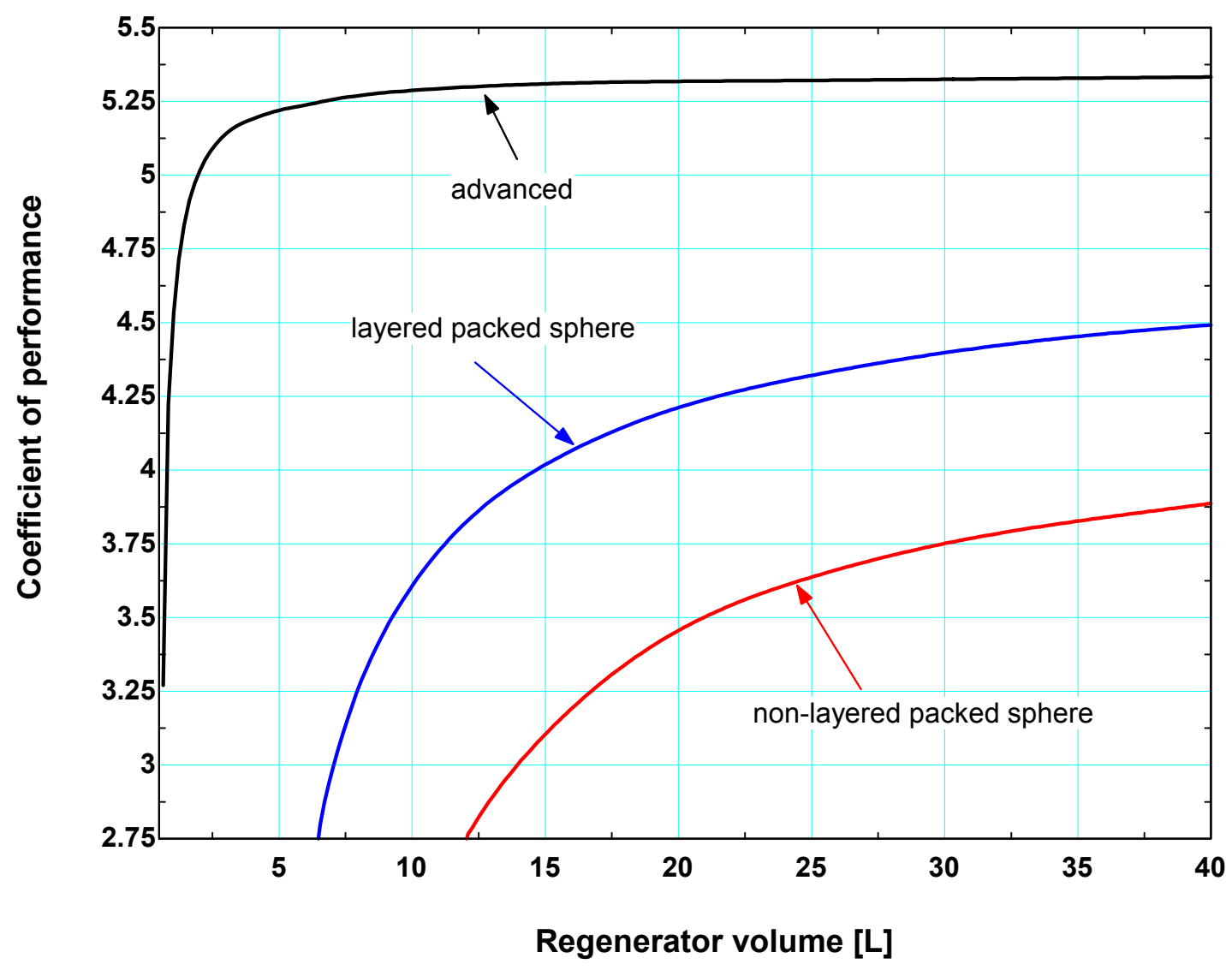

Figure 5.5. COP of an advanced regenerator and layered and nonlayered packed sphere regenerators each capable of producing $8.76 \mathrm{~kW}$ of refrigeration at its optimal aspect ratio.

\subsection{Sensitivity Analysis}

It is important to quantify the dominant losses in a well-designed AMRR system. This information can be used to assess risk areas in modeling, direct additional research, and provide insight into more advanced regenerator designs. The loss of performance that can be associated with each entropy generation mechanism have been individually assessed by "turning off" that specific loss mechanism in the model while leaving all other losses on. The change in the performance of the system that results from the 
deactivation of that particular loss mechanism provides a measure of its importance. This analysis is valid to the extent that the major loss mechanisms do not interact.

The major loss mechanisms in an AMRR for space conditioning that are included in the model developed for this project include pumping losses and losses due to viscous dissipation, axial conduction due to dispersion, axial conduction due to the static conduction through the regenerator bed (solid and fluid), heat transfer over a finite temperature difference in the regenerator, and heat exchanger losses in the hot and cold heat exchanger. The nominal AMRR design point used to carry out the parametric study is design case A from section 4.4.2 which is a spherical layered bed; the inputs for the design case are given in Table 5.2.

Table 5.2. Parameters used for space cooling parametric study.

\begin{tabular}{|l|l|l|l|}
\hline Parameter & Value & Parameter & Value \\
\hline heat rejection temp. & $308.2 \mathrm{~K}$ & hot heat exchanger UA & $1430 \mathrm{~W} / \mathrm{K}$ \\
\hline load temperature & $299.8 \mathrm{~K}$ & motor efficiency & 0.9 \\
\hline cooling capacity & $8.76 \mathrm{~kW}$ & pump efficiency & 0.7 \\
\hline maximum applied field & $1.5 \mathrm{Tesla}$ & heat transfer fluid & Water \\
\hline cold air mass flow rate & $0.57 \mathrm{~kg} / \mathrm{s}$ & number of beds & 6 \\
\hline hot air mass flow rate & $1.42 \mathrm{~kg} / \mathrm{s}$ & period & $0.2 \mathrm{sec}(5 \mathrm{~Hz})$ \\
\hline cold heat exchanger UA & $877.3 \mathrm{~W} / \mathrm{K}$ & sphere size for packing & $0.2 \mathrm{~mm}$ \\
\hline aspect ratio $(L / d)$ & 0.25 & regenerator volume & $10 \mathrm{~L}$ \\
\hline
\end{tabular}

Each loss mechanism was sequentially deactivated within the model and the mass flow rate was adjusted so that the same refrigeration power was provided. The change in the required input power indicates the work savings or the improvement in performance. The results are listed in Table 5.3; note that the input power to the fans was not considered in this study. In Table 5.3, the work savings that results from turning off each loss 
mechanism can be considered to be the amount of power input that was required to overcome the particular loss mechanism.

Table 5.3. Parametric study results for space cooling.

\begin{tabular}{|l|c|c|c|}
\hline case & COP & work input (kW) & work savings (kW) \\
\hline baseline & 5.18 & 1.69 & \\
\hline friction factor = 0 & 6.57 & 1.33 & 0.36 \\
\hline axial dispersion=0 & 5.48 & 1.60 & 0.09 \\
\hline static bed conductivity = 0 & 5.53 & 1.58 & 0.11 \\
\hline perfect heat transfer in bed & 6.52 & 1.34 & 0.35 \\
\hline $\begin{array}{l}\text { cold heat exchanger } \\
\text { effectiveness = 1 }\end{array}$ & 5.94 & 1.47 & 0.22 \\
\hline $\begin{array}{l}\text { hot heat exchanger } \\
\text { effectiveness = 1 }\end{array}$ & 6.33 & 1.38 & 0.31 \\
\hline
\end{tabular}

The Carnot coefficient of performance for a cooler operating between the cold and hot reservoirs specified for this application is 35.69 , which corresponds to a work input of $0.25 \mathrm{~kW}$. Subtracting the total work savings that is associated with all of the loss mechanisms $(1.43 \mathrm{~kW})$ from the baseline work input $(1.69 \mathrm{~kW})$ yields $0.26 \mathrm{~kW}$, which is very close to the Carnot work input. A source of entropy generation that cannot be easily turned off in the model is fluid mixing in the reservoirs - the entropy generated as fluid that is colder than the cold reservoir temperature is introduced to the cold reservoir during the hot-to-cold flow process and the same phenomenon during the cold-to-hot flow process. Therefore, this mechanism is not quantified in this sensitivity study; however, this mixing loss is also inherent to the AMRR cycle modeled in this project which is composed of separated magnetization/demagnetization and flow processes.

Table 5.3 indicates that the dominant losses in the AMRR system are related to pumping losses due to pressure drop across the regenerator and imperfect heat transfer within the regenerator. Conduction (static and dispersive) within the regenerator is also important 
as are the heat transfer losses within hot and cold heat exchangers. The relative equivalence of these losses is likely due to the optimization procedure that was carried out in order to develop design point A. Note that the dominance of the internal regenerator losses explains the performance improvement that can be obtained by using advanced regenerator configurations, as shown in the previous section. Also, as the losses within the bed are reduced the amount of heat that must be rejected is reduced and so the loss associated with the hot heat exchanger, which is also large as shown by Table 5.3 , can be reduced indirectly.

The sensitivity of the model to the various correlations and material properties that were used in its development was investigated using the same AMRR design point described in Table 5.2. Each input was changed by $20 \%$ and the change in the work input required by the cycle in order to provide the fixed refrigeration load was recorded. Changes in the predicted output for variations in each input are given in Table 5.4. In the table, $\Delta$ work input is the change in required input power resulting from a $20 \%$ change in the corresponding parameter.

Table 5.4. Model sensitivity to correlations and material properties.

\begin{tabular}{|l|c|c|c|c|}
\hline parameter & COP & work input (kW) & $\Delta$ work input (kW) & $\Delta$ work input (\%) \\
\hline baseline & 5.18 & 1.69 & \multicolumn{2}{|c|}{} \\
\hline Nusselt number & 5.46 & 1.61 & -0.09 & $-5.10 \%$ \\
\hline friction factor & 4.96 & 1.76 & 0.07 & $4.31 \%$ \\
\hline axial dispersion & 5.12 & 1.71 & 0.02 & $1.19 \%$ \\
\hline static bed conductivity & 5.11 & 1.71 & 0.02 & $1.28 \%$ \\
\hline fluid properties & 5.35 & 1.64 & -0.06 & $-3.30 \%$ \\
\hline $\begin{array}{l}\text { magnetic material } \\
\text { properties }\end{array}$ & 5.55 & 1.58 & -0.11 & $-6.67 \%$ \\
\hline
\end{tabular}




\subsection{Identification of Risk Areas}

Risk areas can be divided into two categories: losses that have been modeled but may be modeled incorrectly (e.g., errors or uncertainty in the heat transfer correlations) and losses that were not modeled.

\subsubsection{Modeled Phenomena}

Table 5.4 shows that the model is most sensitive to the Nusselt number correlation. An error of $20 \%$ in the Nusselt number correlation will lead to a $5.1 \%$ error in the predicted work input to the AMRR. The friction factor correlation is the next most influential correlation. Inaccuracies in the predictions of the Nusselt number or the friction factor in the bed can lead to significant errors in the predicted performance. Table 5.4 shows that axial dispersion and static thermal conductivity of the bed have a smaller impact on the predicted performance but can have a noticeable impact on model predictions.

Table 5.4 also shows that the material properties are an important input to the model. The fluid properties can have a significant impact on performance, but because the properties of most heat transfer fluids are well known and the polynomials used to fit them are highly accurate, the material properties are a low risk model input. The properties of the magnetic material have approximately double the impact of the fluid properties. There are several sources of error associated with the magnetic material properties which make these properties a larger modeling risk than the fluid properties. Experimentally determined magnetic material properties are interpolated with respect to temperature and magnetic field using a spline technique; properties are only available for 
0, 1, 2 and 5 Tesla magnetic fields. This double interpolation of data that is only available on a relatively coarse magnetic field grid may lead to errors in the material properties evaluated at the midpoints of the grid. In a layered bed, it is assumed that the magnitude and shape of the magnetic material properties for each alloy are constant but shifted in relation to the Curie temperature as the Curie temperature is shifted along the bed. This is not the case for all families of magnetic materials and could cause errors in the predicted magnetic material properties.

\subsubsection{Neglected Phenomena}

A number of assumptions were made in the construction of the AMRR model and the possible modeling risk of each assumption is considered here. A list of assumptions made in the model is given in section 2.2, and the assumptions that could have the greatest impact on model predictions are discussed below.

- Seal drag - an AMRR using a rotating bed and stationary magnet will need a dynamic seal in the flow distribution system. There is drag inherent in any dynamic seal and the power necessary to overcome the seal drag may be significant.

- Flow maldistribution - Nusselt number, friction factor, and dispersion correlations assume well distributed flow in the regenerator. A regenerator with a relatively small radius of curvature, a very low fluid flow rate, or other factors that cause poor flow distribution will exhibit behavior that significantly differs from correlations and result in bypass flows that are not modeled by the onedimensional regenerator model. 
- Lumped capacitance correction for high dwell ratios - the lumped fluid heat capacity correction factor was developed with zero dwell time between flow cycles (i.e., no zero flow periods), but dwell times in a practical regenerator may be significant. Interaction between the magnetic material and the heat transfer fluid during zero flow periods may cause errors in the lumped capacity correction factor which will cause errors in the calculated heat transfer coefficients between the regenerator bed and the fluid.

- Eddy currents in regenerator material - as the magnetic material in the regenerator enters and exits the magnetic field eddy currents are generated. The regenerator is heated by the dissipation of the induced eddy currents, which may significantly affect the system performance.

- Magnetic hysteresis - magnetic materials generally exhibit property hysteresis during magnetization and demagnetization. For Gadolinium and its alloys, the hysteresis is very small, but for compounds such as $\mathrm{La}\left(\mathrm{Fe}_{\mathrm{x}} \mathrm{Si}_{1-\mathrm{x}}\right)_{13} \mathrm{H}_{\mathrm{y}}$, the effect can cause significant irreversibilities in the cycle.

- Time varying boundary conditions - due to changes in the ambient temperature, changes in the operating condition and refrigeration load, as well as finite heat capacity of the hot and cold heat exchangers, the temperature of the air entering the heat exchangers and the temperature of the fluid in the AMRR reservoirs may not be constant in time. This effect may affect performance and has not been considered in this project.

- Pressure drop through external passages - pumping loss due to pressure drop is a significant loss mechanism in the AMRR. The current model does not account 
for pressure drop in the heat transfer fluid across the heat exchangers or through external fluid routing passages and flow control valves. The pump power required to move the fluid through these restrictions may be significant.

- Edge effects - because the magnet gap in a practical AMRR is likely to be relatively small. The small height relative to the width of the flow channel may make edge effects considerable. Porosity varies near the walls and flow distributions change, which could cause errors in correlations such as Nusselt number and friction factor.

The phenomena described in this section are not explicitly accounted for in the current model but could play an important role in practical AMRR systems. Experimentation with a passive regenerator presented in Chapter 3 showed good agreement with the predicted results which suggests that none of the effects listed above cause significant modeling errors for the passive case. However, effects associated with magnetization and demagnetization could not be investigated using the passive regenerator test bed. Also, the bed geometry in a practical AMRR may differ significantly from the passive regenerator bed that was tested. 


\section{Chapter $6 \quad$ Conclusions}

Magnetic cooling has the potential to become a practical alternative to current vapor compression refrigeration technology. The metallic refrigerant has essentially zero vapor pressure and therefore is ecologically sound with no Ozone Depletion Potential and zero direct Global Warming Potential. Design studies, presented in this thesis have shown that the active magnetic regenerative refrigeration cycle implemented with materials and geometries that are practical in the near-term have the potential to achieve efficiencies that are competitive with vapor compression technology provided sufficient regenerator volume is used. It should be noted that the regenerator volume required is substantially larger than the compressor which currently can be used to activate a vapor compression system. Therefore, even without considering the additional volume associated with the magnet and motor, these near-term AMRR systems are almost sure to be larger than a comparable vapor compression system. However, studies presented in this thesis also show that advances in magnetocaloric materials and regenerator geometry have the potential to result in a dramatic reduction in the volume of regenerator required and therefore may allow magnetic coolers to compete with vapor compression technology on a longer-term basis.

The most promising magnetic cooler is currently a rotary Active Magnetic Regenerative Refrigerator (AMRR) consisting of a layered regenerator bed. The numerical model presented in this thesis is capable of predicting the performance of a layered AMRR subjected to a temporally varying magnetic field and fluid mass flow. The model treats 
the regenerator bed as a one-dimensional matrix of magnetic material with a spatial variation in Curie temperature. The numerical model uses a fully implicit (in time and space) discretization of the governing energy equations. The resulting set of linearized equations is solved using sparse matrix decomposition in Matlab. The nonlinear aspects of the governing equations (e.g., temperature dependent fluid and magnetic property variations) are handled using a relaxation technique and so iteration is required to obtain the temperature profiles in the regenerator bed. The numerical model of the regenerator bed is coupled to models of auxiliary equipment such as heat exchangers, pumps, and motors in order to predict the performance of an AMRR in typical cooling applications. The cold heat exchanger model is also capable of predicting the loads related to moisture removal for space conditioning applications.

The model predictions were verified experimentally in the passive case against experimental data taken at the Astronautics Corporation in Madison, WI. A packed sphere regenerator consisting of a stainless steel matrix was tested at various mass flow rates, dwell ratios, and temperature spans. Model predictions showed good agreement with experimental data and so these experiments indicate that the numerical model is accurate in this passive limit.

Design studies show that a packed sphere regenerator consisting of layered and nonlayered alloys of gadolinium and erbium can achieve performance that is competitive with vapor compression technology for space cooling applications. The space cooling 
design study was performed at the baseline conditions used by the DOE/ORNL Heat Pump Design Model mark VI and the results from the numerical model were compared to the DOE/ORNL model predictions for a vapor compression air conditioner. In order to produce the same cooling load and achieve the same coefficient of performance as the vapor compression system, the layered regenerator required a volume of approximately 7.5 $\mathrm{L}$ and the non-layered bed required a volume of $15 \mathrm{~L}$.

The predicted performance of an AMRR in a refrigeration application is less attractive when compared to vapor compression systems. An ethylene glycol and water mixture must be used as the heat transfer fluid for this application due to the low temperatures. A non-layered regenerator bed was found to be unable to produce the desired $150 \mathrm{~W}$ load at a coefficient of performance equal to current vapor compression systems. A layered bed was able to match performance of a vapor compression refrigerator with a regenerator volume of approximately $1.3 \mathrm{~L}$. The refrigeration study shows that as the temperature span of the hot and cold reservoirs increases, the benefit derived from a layered bed increases.

This packed sphere regenerator with the erbium and gadolinium alloys represents a relatively near term technology; the Erbium/Gadolinium alloy has been fabricated and tested and can be made in spherical form. An advanced regenerator geometry (parallel plates) composed of an advanced material (Lanthanum Iron Silicon Hydride) at an elevated frequency ( $15 \mathrm{~Hz}$ vs $5 \mathrm{~Hz}$ for the previous) was studied to that may become 
available assess the long-term promise of magnetic refrigeration technology. The advanced regenerator showed significant improvements in performance, both in terms of the volume of regenerator material required and the coefficient of performance. The advanced regenerator produced the desired cooling load at a higher efficiency than the vapor compression system with a regenerator volume of $0.65 \mathrm{~L}$, which is significantly lower that a packed sphere regenerator consisting of layered gadolinium and erbium. The advanced regenerator study shows both the potential of the technology and the need for improved materials and geometries.

\section{Recommendations for Future Work}

The following recommendations relate to performance factors that were not or could not be appropriately modeled, due to lack of information. Additional data are needed to determine the effect of these factors on AMMR peformance.

- Test seal drag on the prototype AMR at Astronautics by measuring motor torque needed to turn the rotary regenerator in the absence of a magnetic field.

- Quantify pressure drop through external passages experimentally at Astronautics. The pressure drop data could be correlated and added to the model to account for all pressure drop in the system.

- Study the effect of dwell ratio on the lumped capacitance correction factor. The model used to develop the lumped capacitance correction factor could be modified to include dwell ratio as a parameter.

- Study edge effects in a practical residential regenerator bed. As the dimensions of a practical regenerator design become more established, edge effects can be 
studied in more depth in the literature. Experiments could also be performed at the passive regenerator test setup at Astronautics to determine how regenerator dimensions affect performance. 


\section{$\underline{\text { References }}$}

R. A. Ackermann, 1997, Cryogenic Regenerative Heat Exchaners, Plenum Press, New York.

J. A. Barclay, O. Moze, and L. Paterson, "A reciprocating magnetic refrigerator for 2-4

Koperation - initial results", Journal of Applied Physics, 50:5870, (1979).

D. A. Beard, "Taylor Dispersion of a Solute in a Microfluidic Channel," Journal of Aplied Physics, vol. 89, 4667 (2001).

P. E. Blumenfeld, F. C. Prenger, A. Sternberg, and C. Zimm, "High Temperature

Superconducting Magnetic Refrigeration", Advances in Cryogenic Engineering, 47:1019, 2002.

J. E. Braun, S. A. Klein, J. W. Mitchell, "Effectiveness Models for Cooling Towers," ASHRAE Transactions: Vol. 95 part 2, 1989 p164-174.

Y. Chen, F. Wang, B. Shen, F. Hu, J. Sun, G. Wang, and Z. Cheng, "Magnetic properties and magnetic entropy change of $L a F e_{11.5} S_{1.5} H_{y}{ }^{\prime \prime}$. J. Phys.: Condens. Matter,

15:7,L161, 2003.

G. D. Dragutinovic, and B. S. Baclic, 1998, Operation of Counterflow Regenerators, Computational Mechanics Inc., Billerica, $M A$.

S. Ergun, "Fluid Flow through Packed Column," Chemical Engineering Progress, Vol. 48, No. 2, 1952, p. 125-127.

S. Fujieda, Y. Hasegawa, A. Fujita, and K. Fukamichi, "Thermal Transport Properties of Magnetic Refrigerants $\mathrm{La}\left(\mathrm{Fe}_{\mathrm{x}} \mathrm{Si}_{1-\mathrm{x}}\right)_{13}$ and Their Hydrides, and $\mathrm{Gd}_{5} \mathrm{Si}_{2} \mathrm{Ge}_{2}$ and MnAs," Journal of Applied Physics, vol. 95 no. 5 (2004).

A. Fujita, S. Fujieda, Y. Hasegawa, and K. Fukamichi, "Itinerant-Electron Metamagnetic Transition and Large Magnetocaloric Effects in $\mathrm{La}\left(\mathrm{Fe}_{\mathrm{x}} \mathrm{Si}_{1-\mathrm{x}}\right)_{13}$ Compounds and Their Hydrides," Physical Review B no. 67, 104416 (2003) 
A. Gan, 1998, M.S. Thesis, Mechanical Engineering, University of Wisconsin Madison, "The Design of Better Household Refrigerators with Improved Energy Consumption."

W. F. Giauque, and D. P. MacDougall, "Attainment of temperatures below $1^{\circ}$ absolute by demagnetization of $\mathrm{Gd}_{2}\left(\mathrm{SO}_{4}\right)_{3} 8 \mathrm{H}_{2} \mathrm{O}^{\prime \prime}$, Phys. Rev., 43:7768, 1933.

E. A. Guggenheim, Thermodynamics, an Advanced Treatment for Chemists and Physicists, North Holland, Amsterdam, 1967.

G.R. Hadley, "Thermal Conductivity of Packed Metal Powders," International Journal of Heat and Mass Transfer, Vol. 29, No.6, 1986, p. 909-919.

F. Hu, B. Shen, J. Sun, and Z. Cheng, "Influence of negative lattice expansion and metamagnetic transition on magnetic entropy change in the compound $\mathrm{LaFe}_{11.4} \mathrm{Si}_{1.6}$ ", Applied Physics Letters, Vol. 78, No. 23, pg. 3675, 2001.

F. P. Incropera, and D. P. DeWitt, Fundamentals of Heat and Mass Transfer, John Wiley \& Sons, New York (1996).

Y. Jaluria, and K. Torrance, 2002, Computational Heat Transfer, $2^{\text {nd }}$ Edition, Taylor and Francis, New York.

D. Jaehnig, 1999, M.S. Thesis, Mechanical Engineering, University of Wisconsin Madison, "A Semi-Empirical Method for Modeling Reciprocating Compressors in Residential Refrigerators and Freezers."

C.P Jeffreson, "Prediction of Breakthrough Curves in Packed Beds," AIChE Journal, Vol. 18, No.2, Mar. 1972, p. 409.

D. L. Johnson, "Reciprocating Magnetic Refrigerator", Proceedings of the $3^{\text {rd }}$ Cryocooler Conference, Boulder, CO, September 17-18, pg. 33, 1984.

M. Kaviany, 1995, Principles of Heat Transfer in Porous Media, Springer, New York, NY, p. 33, 46-47, 130, 228-229.

W. M. Kays and A. L. London, Compact Heat Exchangers, McGraw-Hill Book Company, New York (1964). 
T. T. King, B. A. Rowlett, R. A. Ramirez, P. J. Shirron, E. R. Canavan, M. J. DiPirro, J. S. Panek, J. G. Tuttle, R. D. Shull, and R. A. Fry, "Rare-Earth Garnets and Perovskites for Space-Based ADR Cooling at High T and Low H", Advances in Cryogenic Engineering, 47:1191, 2002.

D. Kunii and J. M. Smith, "Heat Transfer Characteristics of Porous Rocks," AIChE Journal: 71, 1960.

A. F. Lacaze, A. A. Lacaze, R. Beranger, and G. Bon Mardion, "Thermodynamic analysis of a double-acting reciprocating magnetic refrigerator", Proceedings of the $9^{\text {th }}$ International Cryogenic Engineering Conference, Kobe, Japan, May 11-14, pg. 14, 1982.

K. Matsumoto, and T. Hashimoto, "Thermodynamic analysis of magnetically active regenerator", Proceedings of the International Congress of Cryogenics and Refrigeration, Hangzhou, China, May 22-26, pg. 110, 1989.

G.F. Nellis and S.A. Klein, "Regenerative heat exchangers with significant entrained fluid heat capacity," submitted to the International Journal of Heat and Mass Transfer, July 2004.

G. F. Nellis, and J. L. Smith, Jr., 1998, “An Experimental GM/Magnetic Refrigerator”, Advances in Cryogenic Engineering, Vol. 43, pp. 1767-1774.

T. Numazawa, H. Kimura, M. Sato, and H. Maeda, "Analysis of a magnetic refrigerator operating temperature between $10 \mathrm{~K}$ and $1.4 \mathrm{~K}^{\prime \prime}$, Proceedings of the $4^{\text {th }}$ International Cryocoolers Conference, Easton, MD, September 25-26, pg. 79, 1986.

G. Patton, G. Green, J. Stevens, and J. Humphrey, "Reciprocating Magnetic Refrigerator", Proceedings of the $4^{\text {th }}$ International Cryocooler Conference, Easton, MD, September 25-26, pg. 65, 1986.

V. K. Pecharsky, and K. A. Gschneidner, Jr., "Giant Magnetocaloric Effect in $\mathrm{Gd}_{5}\left(\mathrm{Si}_{2} \mathrm{Ge}_{2}\right)$ ", Physical Review Letters, Vol. 78, No. 23, pg. 4494, 1997.

K. Rice, DOE/ORNL Heat Pump Design Model, Mark V \& VI Version, http://www.ornl.gov/ wlj/hpdm/ 
W. M. Rohsenow, J. P. Hartnett and Y. I. Cho, 1998, Handbook of Heat Transfer, McGraw-Hill, New York, NY.

P. Seyfert, P. Bredy, and G. Claudet, "Construction and testing of a magnetic refrigeration device for the temperature range of 5 to $15 K^{\prime \prime}$, Proceedings of the $12^{\text {th }}$ International Cryogenic Engineering Conference, Southampton, UK, July 12-15, pg. 607, 1988.

$W$. A. Steyart, "Rotating Carnot-cycle magnetic refrigerators for use near $2 K^{\prime \prime}$, Journal of Applied Physics, 49:1227, 1978.

C. P. Taussig, G. R. Gallagher, J. L. Smith, Jr., and Y. Iwasa, "Magnetic refrigeration based on magnetically active regeneration", Proceedings of the $4^{\text {th }}$ International Cryocoolers Conference, Easton, MD, September 25-26, pg. 79, 1986.

O. Tegus, E. Bruck, K. H. Buschow, Jr., and F. R. de Boer, Nature, 415:150, 2002.

K. Vafai, 2000, Handbook of Porous Media, Marcel Dekker, Inc, New York, NY, p. 235237.

H. Wada, and Y. Tanabe, Appl. Phys. Lett., 79:3302, 1999.

N. Wakao and S. Kaguei, 1982, Heat and Mass Transfer in Packed Beds, Gordon and Breach Science Publishers, New York, NY, chapter 8.

W. Wu, "Room Temperature Magnetic Refrigerator using a 1.4 T Permanent Magnet Field Source", Abstract K7.004, American Physical Society Conference, Austin, TX, 2003.

C. B. Zimm, A. Sternberg, A. G. Jastrab, A. M. Boeder, L. M. Lawton, Jr., and J. J. Chell, 2002, "Rotating Bed Magnetic Refrigeration Apparatus", U.S. Patent Application, US 2002/0053209 A1. 


\section{Appendix A: Matlab Code}

\section{Regenerator Bed Model}

function[xr,tr,Tr,xf,tf,Tf,Tfn,Trn,mdot,muoH,TCurie,hf,sf,sr,Tds,hflux,sflux,dQ,dSgen1, Tfa,keff,Nuf,dQfluid,dSgen2, heat_load,ref_load,ref_cap,COP]=AMR_rev5_4_2hx(TC,T

H,mdot_amp,L,D,fluid);

$\% \%$ Bed paramters

$\operatorname{tau}=0.2 ; \% \mathrm{~s}$

muoH_max $=1.5 ; \%$ Tesla

$\mathrm{dh}=0.0002 ; \% \mathrm{~m}$

$\mathrm{Ac}=\mathrm{pi}^{*} \mathrm{D}^{\wedge} 2 / 4 ; \% \mathrm{~m}^{\wedge} 2$

as $=1 / \mathrm{dh} ; \% \mathrm{~m}^{\wedge} 2 / \mathrm{m}^{\wedge} 3$

eps $=0.36$;

$\mathrm{kr}=10.4 ; \% \mathrm{~W} / \mathrm{m}-\mathrm{K}$

bed $=1 ; \% 1=$ A $2=\mathrm{B}$

n_beds $=6$;

arc_mag $=180 ; \%$ degrees

L_mag $=$ arc_mag*n_beds $/ 360^{*} \mathrm{~L}$;

$\mathrm{L}$ ramp $=0.25 * \mathrm{~L}$ _mag; \%distance of magnet that shows a ramp function divided by magnet length

L_flow $=1 * \mathrm{~L} ; \%$ ratio of bed length to bed length in which there is flow

n_motor $=.9 ; \%$ electric motor efficiency

n_pump $=.7 ; \%$ pump efficiency

$\% \%$ Conditions

magfunc $=2 ; \% 1=$ rotating bed, $2=$ constant $m u o H$ across bed $3=$ constant muoH with spinning bed parameters

shwgraphs $=0 ; \% 0=$ don't show graphs $1=$ show graphs

htc $=1 ; \% 1$ for actual numbers, 2 for high value of $\mathrm{Nu}$

magprops $=2 ; \% 1$ for Curie Weiss, 2 for interpolated data, 3 for constant

cond $=1 ; \% 1$ for axial conduction 2 for no axial conduction

layer $=1 ; \% 0$ not layered $1=$ layered

$\mathrm{m}=84$

$\mathrm{n}=60$;

reltol $=0.005$;

$\mathrm{wt}=1$;

$\mathrm{N}=2 * \mathrm{~m} * \mathrm{n}+\mathrm{m}+\mathrm{n}$

$\mathrm{A}=\operatorname{spalloc}(\mathrm{N}, \mathrm{N}, 4 * \mathrm{~N})$;

$\mathrm{B}=\operatorname{spalloc}(\mathrm{N}, 1, \mathrm{~N})$;

modelcheck $=0 ; \% 1=$ show $\mathrm{T}$-s and enthalpy flux graphs $0=$ don't show them

$\mathrm{i}=0: \mathrm{n}$;

$\mathrm{xf}=\mathrm{L} * \mathrm{i} / \mathrm{n}$;

$\mathrm{j}=1: \mathrm{m}$; 
$\mathrm{tf}=\left(\mathrm{j}^{\prime}-0.5\right) * \operatorname{tau} / \mathrm{m}$

mdot=mdotfunction_k(tf,tau,L,mdot_amp,L_flow,n_beds);

$\mathrm{i}=1: \mathrm{n}$;

$\mathrm{xr}=\left(\mathrm{i}^{\prime}-0.5\right) * \mathrm{~L} / \mathrm{n}^{\prime}$;

$\mathrm{j}=0: \mathrm{m}$;

$\operatorname{tr}=j^{\prime} * \operatorname{tau} / \mathrm{m}$

muoH=muoHfunction_k2(xr,tr,tau,L,muoH_max,n_beds,L_ramp,arc_mag,L_mag,magfu nc);

$[$ TCurie $]=$ TCuriefunction $(\mathrm{xr}, \mathrm{L}, \mathrm{TC}, \mathrm{TH}$, layer $)$;

for $\mathrm{j}=0: \mathrm{m}$

$\operatorname{Trg}(:, \mathrm{j}+1)=\mathrm{TH}-\mathrm{xr} *(\mathrm{TH}-\mathrm{TC}) / \mathrm{L}$;

end

for $\mathrm{j}=1: \mathrm{m}$

$\operatorname{Tfg}(:, \mathrm{j})=\mathrm{TH}-\mathrm{xf}^{*}(\mathrm{TH}-\mathrm{TC}) / \mathrm{L}$;

end

$\%$ Calculate lumped cap correction factor

if(strcmp(fluid,'water'))

[muf,kf,cf,rhof,hf,sf]=props_water(Tfg);

elseif(strcmp(fluid,'air'))

[muf,kf,cf,rhof,hf,sf]=props_air(Tfg);

elseif(strcmp(fluid,'water_const'))

[muf,kf,cf,rhof,cfunc, T_ref]=fluidprops(Tfg);

elseif(strcmp(fluid,'eg50'))

[muf,kf,cf,rhof,hf,sf]=props_ethgly50_3(Tfg);

end

[cmuoH,dsdmuoH,rhor,sr]=magmatprops_2k2(Trg,TCurie,muoH);

cf_av $=$ mean $($ mean $(\mathrm{cf}))$;

$\mathrm{cmuoH}$ av $=$ mean $(\operatorname{mean}(\mathrm{cmuoH}))$;

$\mathrm{R}=($ rhof*eps*cf_av $) /\left(\right.$ rhor* $(1-\mathrm{eps}) * \mathrm{cmuoH} \_$av $)$;

$\mathrm{AF}=1+1.764 * \mathrm{R}+1.0064 * \mathrm{R}^{\wedge} 2 ; \%$ correction factor

done $=0$;

while $($ done $==0)$

if(strcmp(fluid,'water'))

[muf,kf,cf,rhof,hf,sf]=props_water(Tfg);

elseif(strcmp(fluid,'air'))

[muf,kf,cf,rhof,hf,sf]=props_air(Tfg);

elseif(strcmp(fluid,'water_const'))

[muf,kf,cf,rhof,cfunc,T_ref]=fluidprops(Tfg);

elseif(strcmp(fluid,'eg50'))

[muf,kf,cf,rhof,hf,sf]=props_ethgly50_3(Tfg);

end

for $\mathrm{i}=1: \mathrm{n}$ 


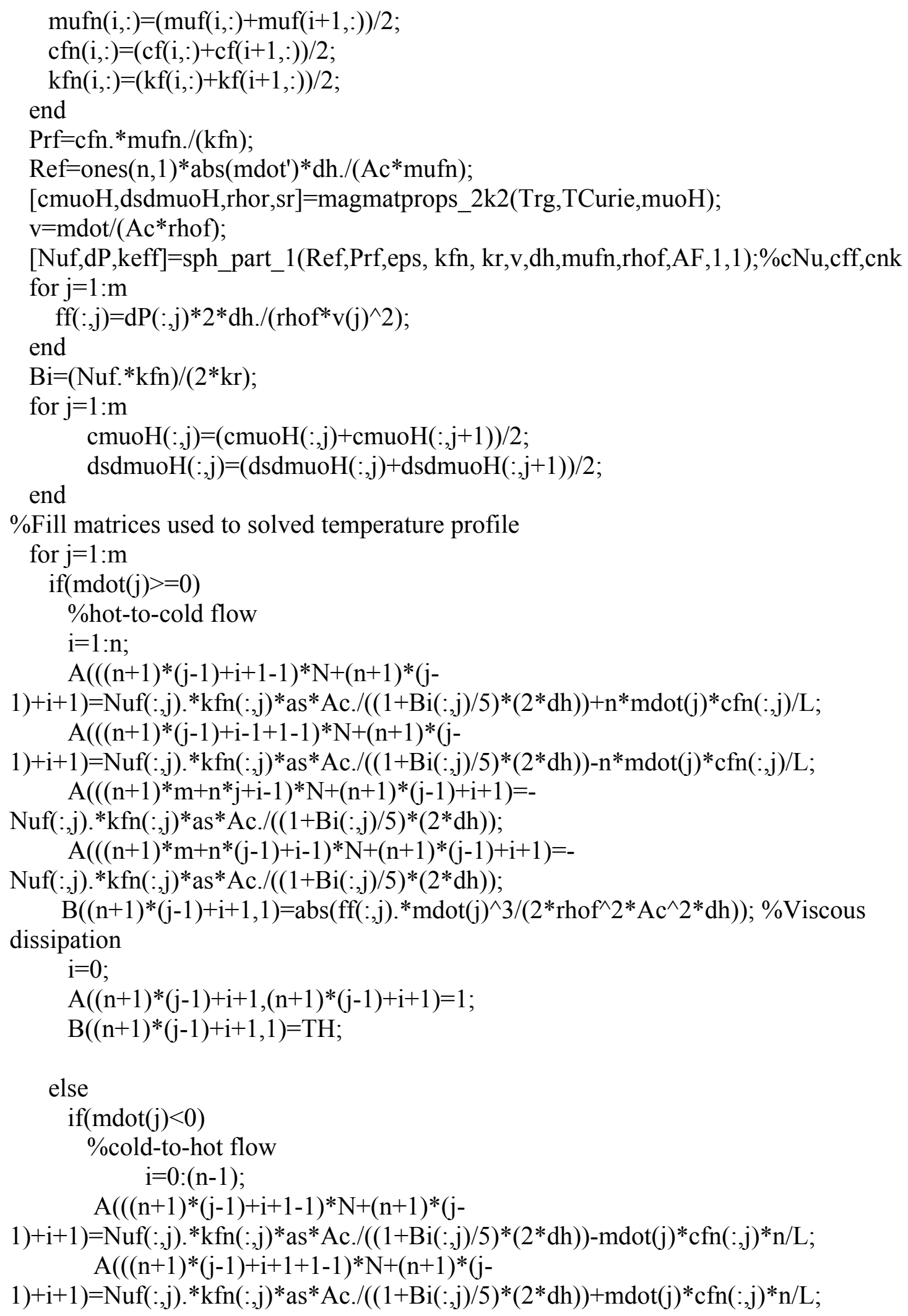




$$
\mathrm{A}(((\mathrm{n}+1) * \mathrm{~m}+\mathrm{n} * \mathrm{j}+\mathrm{i}+1-1) * \mathrm{~N}+(\mathrm{n}+1) *(\mathrm{j}-1)+\mathrm{i}+1)=-
$$

$\left.\operatorname{Nuf}(:, \mathrm{j}) .{ }^{*} \operatorname{kfn}(: \mathrm{j}) * a\right)^{*}$ Ac. $/((1+\operatorname{Bi}(:, \mathrm{j}) / 5) *(2 * \mathrm{dh}))$;

$\mathrm{A}(((\mathrm{n}+1) * \mathrm{~m}+\mathrm{n} *(\mathrm{j}-1)+\mathrm{i}+1-1) * \mathrm{~N}+(\mathrm{n}+1) *(\mathrm{j}-1)+\mathrm{i}+1)=-$

$\operatorname{Nuf}(:, \mathrm{j}) .{ }^{*} \mathrm{kfn}(: \mathrm{j}) *$ as $*$ Ac. $/((1+\mathrm{Bi}(:, \mathrm{j}) / 5) *(2 * \mathrm{dh}))$ dissipation

$B((n+1) *(j-1)+i+1,1)=\operatorname{abs}\left(f f(:, j) . * \operatorname{mdot}(j) \wedge 3 /\left(2 * \operatorname{rhof}^{\wedge} 2 * \operatorname{Ac}^{\wedge} 2 * d h\right)\right) ; \%$ viscous

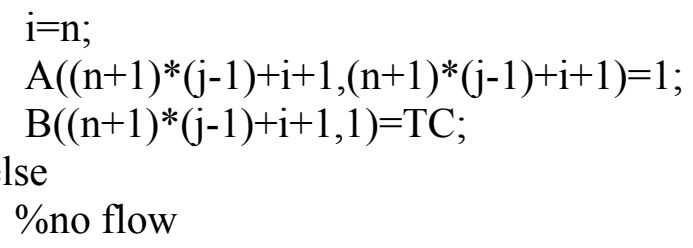


$\mathrm{A}(((\mathrm{n}+1) * \mathrm{~m}+\mathrm{n} * \mathrm{j}+\mathrm{i}-$

$\left.1)^{*} \mathrm{~N}+(\mathrm{n}+1)^{*} \mathrm{~m}+\mathrm{n}^{*} \mathrm{j}+\mathrm{i}\right)=\mathrm{Nuf}\left(\mathrm{i}^{\prime}, \mathrm{j}\right) .{ }^{*} \mathrm{kfn}\left(\mathrm{i}^{\prime}, \mathrm{j}\right) *$ as $*$ Ac. $/\left(\left(1+\mathrm{Bi}\left(\mathrm{i}^{\prime}, \mathrm{j}\right) / 5\right) *(2 * \mathrm{dh})\right)+$ Ac* $($ rhof*eps $*$

cfn(i',j)+(1-eps)*rhor*cmuoH(i',j))*m/tau+n^2*keff(i',j)*Ac/(2* $\left.\mathrm{L}^{\wedge} 2\right) ;$

$\mathrm{A}(((\mathrm{n}+1) * \mathrm{~m}+\mathrm{n} *(\mathrm{j}-1)+\mathrm{i}-$

$1) * \mathrm{~N}+(\mathrm{n}+1) * \mathrm{~m}+\mathrm{n} * \mathrm{j}+\mathrm{i})=\mathrm{Nuf}\left(\mathrm{i}^{\prime}, \mathrm{j}\right) . * \mathrm{kfn}\left(\mathrm{i}^{\prime}, \mathrm{j}\right) * a{ }^{*} \mathrm{Ac} . /\left(\left(1+\mathrm{Bi}\left(\mathrm{i}^{\prime}, \mathrm{j}\right) / 5\right) *(2 * \mathrm{dh})\right)-$

Ac*(rhof*eps*cfn(i',j)+(1-eps)*rhor*cmuoH(i',j))*m/tau+n^2*keff(i',j)*Ac/(2* L $\left.^{\wedge} 2\right)$;

$\mathrm{A}\left(((\mathrm{n}+1) * \mathrm{~m}+\mathrm{n} *(\mathrm{j}-1)+(\mathrm{i}-1)-1) * \mathrm{~N}+(\mathrm{n}+1) * \mathrm{~m}+\mathrm{n}^{*} \mathrm{j}+\mathrm{i}\right)=-\mathrm{n}^{\wedge} 2 * k \operatorname{keff}\left(\mathrm{i}^{\prime}, \mathrm{j}\right) * A c . /\left(2 * \mathrm{~L}^{\wedge} 2\right)$;

$A\left(((n+1) * m+n *(j)+(i-1)-1) * N+(n+1)^{*} m+n * j+i\right)=-n^{\wedge} 2 * k e f f\left(i^{\prime}, j\right) * A c . /\left(2^{*} L^{\wedge} 2\right)$;

end

$\mathrm{j}=0$;

$\mathrm{i}=1: \mathrm{n}$;

$A(((n+1) * m+n * j+i-1) * N+(n+1) * m+n * j+i)=1$;

$A(((n+1) * m+n * m+i-1) * N+(n+1) * m+n * j+i)=-1$;

$\mathrm{X}=\mathrm{A} \backslash \mathrm{B}$;

for $\mathrm{j}=1: \mathrm{m}$

$\mathrm{i}=0: \mathrm{n}$;

$\operatorname{Tf}(:, j)=\operatorname{full}(X((n+1) *(j-1)+i+1))$;

end

for $\mathrm{j}=0: \mathrm{m}$

$\mathrm{i}=1: \mathrm{n}$;

end

$$
\operatorname{Tr}(:, j+1)=f u l l(X((n+1) * m+n * j+i))
$$

$\operatorname{err}=\max (\max (\max (\operatorname{abs}(\operatorname{Tf}-\mathrm{Tfg}))), \max (\max (\operatorname{abs}(\operatorname{Tr}-\operatorname{Trg}))))$

if $($ err $<$ reltol $)$

if(strcmp(fluid,'water'))

[muf,kf,cf,rhof,hf,sf]=props_water(Tf);

elseif(strcmp(fluid,'air'))

[muf,kf,cf,rhof,hf,sf]=props_air(Tf);

elseif(strcmp(fluid,'water_const'))

[muf,kf,cf,rhof,hf,sf]=fluidprops(Tf);

elseif(strcmp(fluid,'eg50'))

[muf,kf,cf,rhof,hf,sf]=props_ethgly50_3(Tf);

end

[cmuoH,dsdmuoH,rhor,sr]=magmatprops_2k2(Trg,TCurie,muoH);

done $=1 ; \%$ Relaxation process complete

else

$\mathrm{Tfg}=\mathrm{Tf} * \mathrm{wt}+\mathrm{Tfg} *(1-\mathrm{wt})$;

$\operatorname{Trg}=\operatorname{Tr}^{*} \mathrm{wt}+\operatorname{Trg}^{*}(1-\mathrm{wt})$;

end $\%$ Update guess temp values

end

for $\mathrm{i}=1: \mathrm{n}$

for $\mathrm{j}=1: \mathrm{m}$

$\operatorname{Tfn}(i, j)=(\operatorname{Tf}(i, j)+\operatorname{Tf}(i+1, j)) / 2$;

$\operatorname{Trn}(\mathrm{i}, \mathrm{j})=(\operatorname{Tf}(\mathrm{i}, \mathrm{j})+\operatorname{Tr}(\mathrm{i}, \mathrm{j}+1)) / 2$;

end 
end

for $\mathrm{i}=1: \mathrm{m}$

if abs(mdot(i) $)<$ mdot_amp

$\operatorname{Tfa}(:, \mathrm{i})=\mathrm{Tfn}(:, \mathrm{i})$;

else

$\mathrm{Tfa}(:, \mathrm{i})=\mathrm{Tfn}(:, \mathrm{i})$;

end

end

Tout_avg=trapz $\left(\mathrm{tf}(1: \mathrm{m} / 2), \operatorname{Tf}(\mathrm{n}+1,1: \mathrm{m} / 2)^{\prime}\right) /(\mathrm{tf}(\mathrm{m} / 2,1)-\mathrm{tf}(1,1))$;

eff $=($ TH-Tout_avg $) /(\mathrm{TH}-\mathrm{TC})$;

$\mathrm{U}=$ mdot_amp ${ }^{*} \mathrm{cf}(1,1) * \operatorname{tau} /(2 * \mathrm{Ac} * \mathrm{~L} *(1-\mathrm{eps}) *$ rhor*cmuoH $(1,1))$;

Crat $=A c^{*} L^{*}(1-e p s) *$ rhor*cmuoH $(1,1) /($ mdot_amp*cf $(1,1) * \operatorname{tau} / 2)$;

$\%$ Calculate penetration depth

$\operatorname{delta}=2 *(\operatorname{tau} / 2 * \mathrm{kr} . /($ rhor* $\mathrm{cmuoH})) . \wedge 0.5$;

$\%$ Calculate regenerator bed capacity

for $\mathrm{i}=1: \mathrm{n}$

$\operatorname{dcapr}(\mathrm{i})=\operatorname{trapz}(\operatorname{tr}, \operatorname{cmuoH}(\mathrm{i},:)) / \operatorname{tau}$;

end

capr $=\operatorname{sum}($ dcapr $) *$ rhor*Ac*L $/ n$;

num $=$ mdot. ${ }^{*} \operatorname{cfn}(\mathrm{n} / 2,:)^{\prime} . /(\mathrm{capr} / \mathrm{tau})$;

$\%$ Calculate parameters for lumped heat capacitance corrections

$\mathrm{V}=\mathrm{Ac}^{*} \mathrm{~L}$;

$\operatorname{crtot}=$ mean $($ mean $(\mathrm{cmuoH}))$;

$\mathrm{cftot}=$ mean $($ mean $(\mathrm{cf}))$;

$\mathrm{Cr}=$ rhor*V*(1-eps $) *$ crtot;

$\mathrm{Cf}=\mathrm{rhof}^{*} \mathrm{~V}^{*}(\mathrm{eps}) *$ cftot;

$\mathrm{R}=\mathrm{Cf} / \mathrm{Cr}$

lambda $=$ tau*L_flow/(L*n_beds);

$\mathrm{U}=$ mdot_amp* ${ }^{*}$ ctot*lamb $\overline{\mathrm{d}} \mathrm{a} /(\mathrm{Cr}+\mathrm{Cf})$

$\mathrm{NTU}=\mathrm{Nuf}(1,19) * \mathrm{kf}(1,19) * \mathrm{Ac}^{*} \mathrm{~L} * \mathrm{as} /(\mathrm{dh} *$ mdot_amp*cf(1,19))

$\%$ Tds check

for $\mathrm{i}=1: \mathrm{n}$

$\operatorname{Tds}(\mathrm{i}, 1)=\operatorname{trapz}(\operatorname{sr}(\mathrm{i},:), \operatorname{Tr}(\mathrm{i},:)) *(1-\mathrm{eps}) *$ rhor*Ac; $\mathrm{dQ}(\mathrm{i}, 1)=\operatorname{Tds}(\mathrm{i}, 1) * \mathrm{~L} / \mathrm{n}$;

end

for $\mathrm{i}=1:(\mathrm{n}+1)$

hflux $(\mathrm{i}, 1)=\operatorname{sum}\left(\right.$ mdot. $^{*}$ hf $\left.(\mathrm{i},:)^{\prime}\right) * \operatorname{tau} / \mathrm{m}$;

$\operatorname{sflux}(\mathrm{i}, 1)=\operatorname{sum}\left(\right.$ mdot. $\left.^{*} \operatorname{sf}(\mathrm{i},:)^{\prime}\right){ }^{*} \mathrm{tau} / \mathrm{m}$;

end

$\%$ Calculate energy loss due to pressure drop across the bed and pumping 


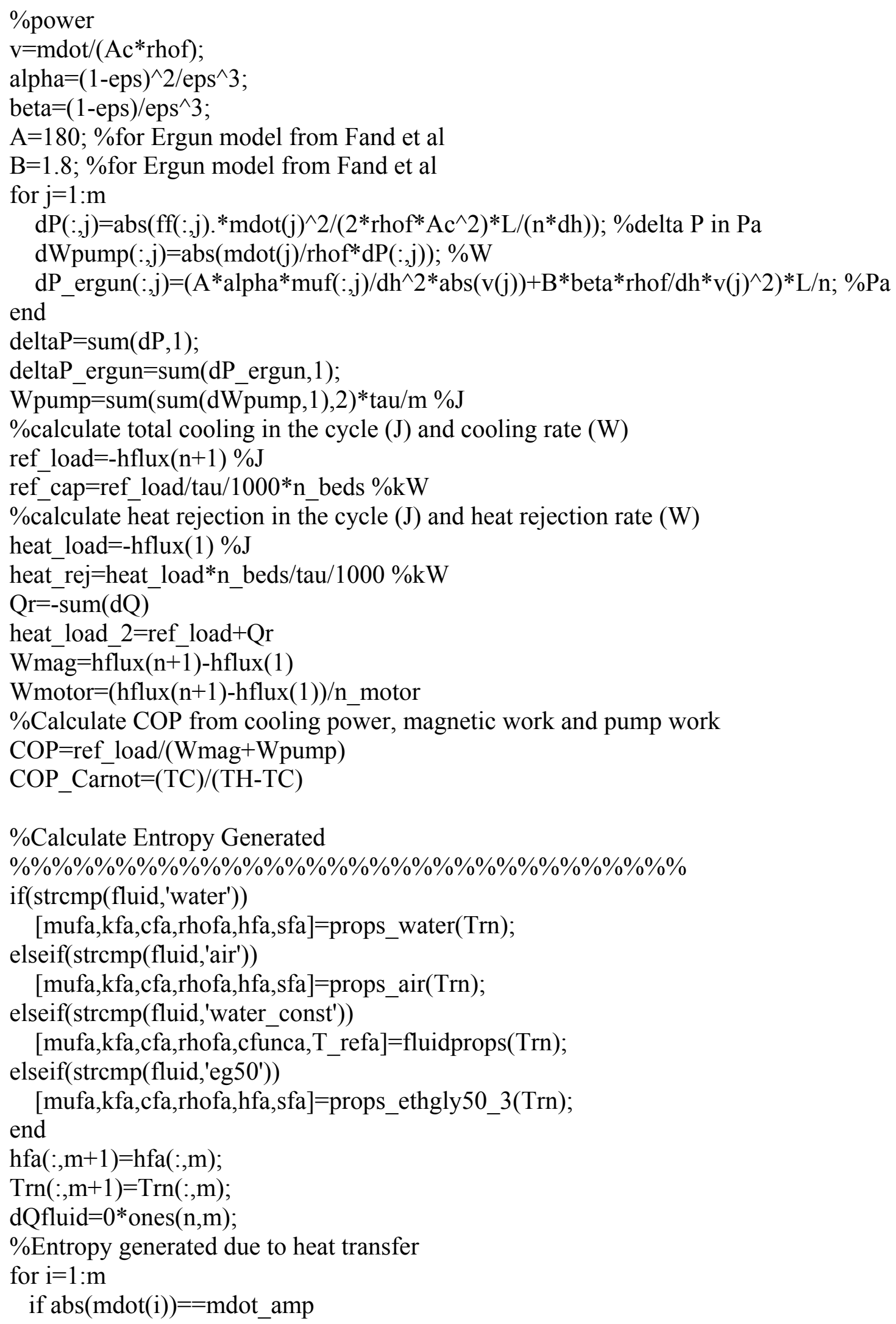


dSgen1(:,i)=Nuf(:,i).*kfn(:,i)*as*Ac/(dh).*(Tfn(:,i)-Trn(:,i)).*(1./Trn(:,i)-1./Tfn(:,i)); $\% \mathrm{~J} / \mathrm{K}$ differential $\mathrm{S}$ gen from bed to fluid heat transfer end

\%\%\%\%\%\%\%\%\%\%\%\%\%\%\%\%\%\%\%\%\%\%\%\%\%\%\%\%\%\%\%\%

$\mathrm{h}(:, \mathrm{i})=\mathrm{Nuf}(:, \mathrm{i}) .{ }^{*} \mathrm{kfn}(:, \mathrm{i}) / \mathrm{dh}$;

heff(:,i) $=\mathrm{h}(:, \mathrm{i}) . /(1+\mathrm{Bi}(:, \mathrm{i}) / 5)$;

dQfluid(:,i) $=(\mathrm{hfa}(:, \mathrm{i}+1)-\mathrm{hfa}(:, \mathrm{i})) *$ eps*Ac*L/n*rhof/(tau/m); \%W change in fluid internal energy during no flow conditiions

$\operatorname{deltaT}(:, \mathrm{i})=\mathrm{dQfluid}(:, \mathrm{i}) /\left(\operatorname{Nuf}(:, \mathrm{i}) .{ }^{*} \mathrm{kfn}(:, \mathrm{i}) * \mathrm{as}^{*} \mathrm{Ac} /(\mathrm{dh}) * \mathrm{~L} / \mathrm{n}\right)$;

dSgen2(:,i)=abs(dQfluid(:,i)).*abs(1./Trn(:,i)-1./(Trn(:,i)+deltaT(:,i))); \%W/K

end

Sgenlumped $=$ sum(sum(dSgen 2,1$), 2) * \operatorname{tau} / \mathrm{m} \% \mathrm{~J} / \mathrm{K}$

\%\%\%\%\%\%\%\%\%\%\%\%\%\%\%\%\%\%\%\%\%\%\%\%\%\%\%\%\%\%\%\%

\%Entropy generated due to axial conduction

for $\mathrm{i}=2: \mathrm{n}-1$

for $\mathrm{j}=2: \mathrm{m}$

Qcond $(\mathrm{i}, \mathrm{j})=\operatorname{Tr}(\mathrm{i}, \mathrm{j}) *\left(\mathrm{n}^{\wedge} 2 * \operatorname{keff}(\mathrm{i}, \mathrm{j}) * \mathrm{Ac} / \mathrm{L}^{\wedge} 2\right)+\operatorname{Tr}(\mathrm{i}, \mathrm{j}-1)^{*}\left(\mathrm{n}^{\wedge} 2 * \operatorname{keff}(\mathrm{i}, \mathrm{j}) * A c /\left(\mathrm{L}^{\wedge} 2\right)\right)-$

$\operatorname{Tr}(\mathrm{i}-1, \mathrm{j}-1) *\left(\mathrm{n}^{\wedge} 2 * \operatorname{keff}(\mathrm{i}, \mathrm{j}) * A c /\left(2 * \mathrm{~L}^{\wedge} 2\right)\right)-\operatorname{Tr}(\mathrm{i}-1, \mathrm{j}) *\left(\mathrm{n}^{\wedge} 2 * \operatorname{keff}(\mathrm{i}, \mathrm{j}) * A c /\left(2 * \mathrm{~L}^{\wedge} 2\right)\right)-\operatorname{Tr}(\mathrm{i}+1, \mathrm{j}-$

$1) *\left(n^{\wedge} 2 * \operatorname{keff}(i, j) * A c /\left(2 * L^{\wedge} 2\right)\right)-\operatorname{Tr}(i+1, j) *\left(n^{\wedge} 2 * \operatorname{keff}(i, j) * A c /\left(2 * L^{\wedge} 2\right)\right) ;$

end

dScond $(\mathrm{i}, 2: \mathrm{m})=\mathrm{Q} \operatorname{cond}(\mathrm{i}, 2: \mathrm{m}) . *(1 . / \operatorname{Tr}(\mathrm{i}+1,2: \mathrm{m})-1 . / \operatorname{Tr}(\mathrm{i}, 2: \mathrm{m}))$;

end

Scond $=\operatorname{trapz}((\mathrm{L} / \mathrm{n} * \operatorname{trapz}(\mathrm{dScond}, 1)), 2) * \operatorname{tau} / \mathrm{m} \% \mathrm{~J} / \mathrm{K}$

Sgentot=heat_load/TH-ref_load/TC\%-(sflux(n+1)-sflux(1))

SgenQ $=\operatorname{sum}((\mathrm{L} / \mathrm{n} * \operatorname{sum}(\mathrm{dSgen} 1,1)), 2) * \operatorname{tau} / \mathrm{m} \% * \mathrm{~L} \% \mathrm{~J} / \mathrm{K}$

Sgenbed $=(\operatorname{sflux}(\mathrm{n}+1)-\operatorname{sflux}(1)) \% \mathrm{~J} / \mathrm{K}$ total entropy generated in the bed

Sgenvisdis=Sgenbed-SgenQ-Scond $\% \mathrm{~J} / \mathrm{K}$ entropy generated by viscous dissipation

SgenmixH=heat_load/TH + sflux $(1) \% \mathrm{~J} / \mathrm{K}$ entropy generated due to mixing in the hot end SgenmixC $=$-ref_load/TC-sflux $(\mathrm{n}+1) \% \mathrm{~J} / \mathrm{K}$ entropy generated due to mixing in the cold end

\section{Heat Exhanger Model}

function[Tcin,Thin,Tmixh,Tmixc,TCR,THR,effc,effh,Qc,Qh,hhin,ref_cap,COP, Tcout, Thout, Tairoutc, Tairouth] $=$ hxmodel5(L,D,mdot_amp,fluid);

fluid

$\mathrm{TH}=308.2$;

$\mathrm{TC}=299.8$;

$\%$ mdot_amp $=0.6 ; \% \mathrm{~kg} / \mathrm{s}$

mdotairh $=1.416 ; \% \mathrm{~kg} / \mathrm{s}$

mdotairc $=0.5651 ; \% \mathrm{~kg} / \mathrm{s}$

cpair $=1027 ; \% \mathrm{~J} / \mathrm{kg}$

Cairh $=$ cpair*mdotairh;

Cairc $=$ cpair*mdotairc; 


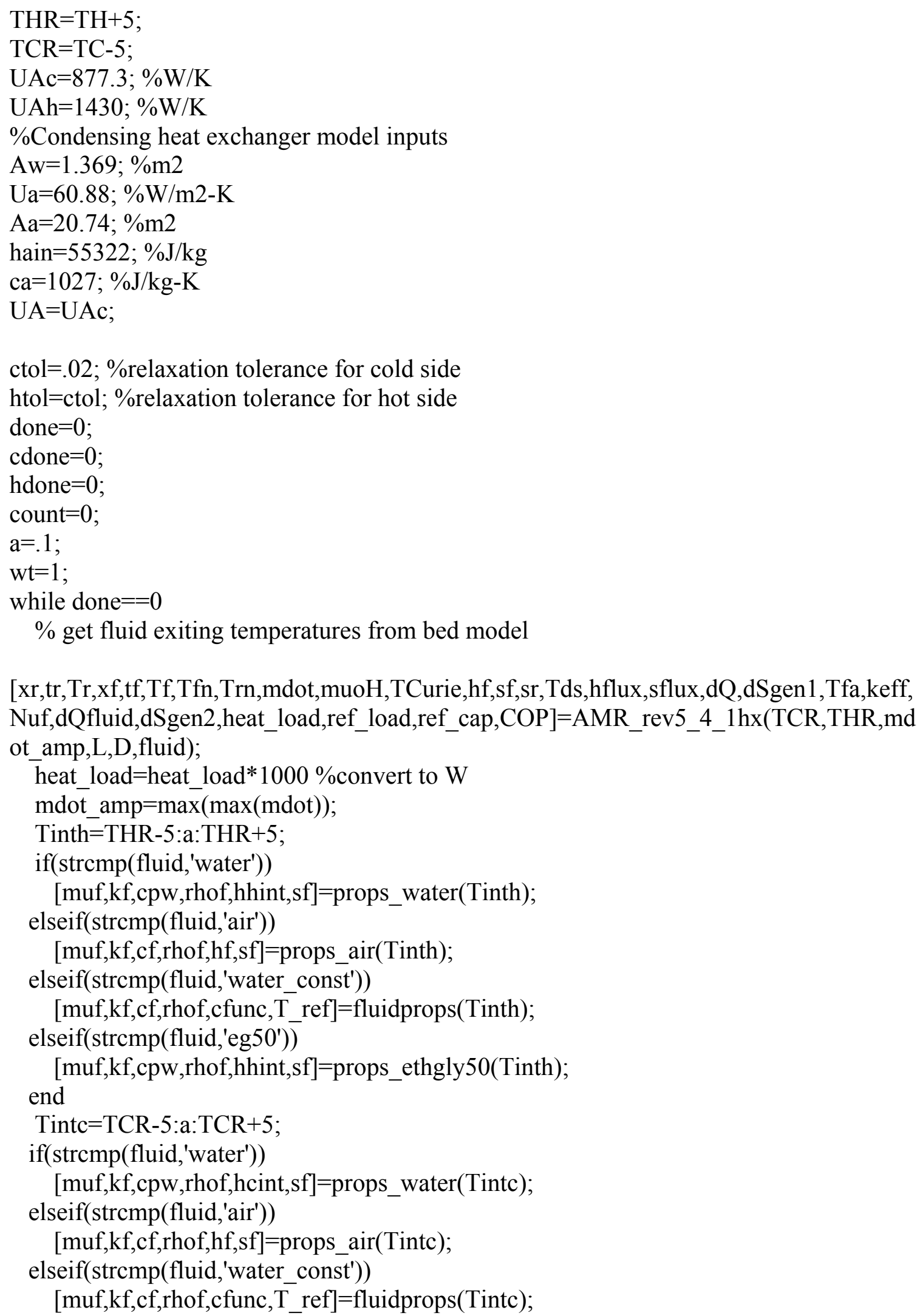


elseif(strcmp(fluid,'eg50'))

[muf,kf,cpw,rhof,hcint,sf]=props_ethgly50(Tintc);

end

$[\mathrm{n}, \mathrm{m}]=\operatorname{size}(\mathrm{Tf})$;

$\mathrm{j}=0$;

$\mathrm{k}=0$;

for $\mathrm{i}=1: \mathrm{m}$

if $\operatorname{mdot}(\mathrm{i})==$ mdot $\_$amp

$\mathrm{j}=\mathrm{j}+1$;

$\operatorname{Tcin}(j)=\operatorname{Tf}(n, i)$;

$h \operatorname{cin}(j)=h f(n, i)$;

elseif $\operatorname{mdot}(\mathrm{i})==-$ mdot_amp

$\mathrm{k}=\mathrm{k}+1$;

$\operatorname{Thin}(\mathrm{k})=\operatorname{Tf}(1, \mathrm{i})$;

$\operatorname{hhin}(\mathrm{k})=\mathrm{hf}(1, \mathrm{i})$;

end

end

\%determine enthalpy average fluid temp entering each $\mathrm{hx}$

hmixc=mean(hcin);

hmixh=mean(hhin);

Tmixc=interp1 (hcin,Tcin,hmixc)

Tmixh=interp1(hhin,Thin,hmixh)

[muf,kf,cpwaterh,rhof,hf,sf]=props_water(Tmixh);

[muf,kf,cpwaterc,rhof,hf,sf]=props_water(Tmixc);

$\mathrm{Cfh}=$ cpwaterh*mdot_amp;

if $\mathrm{Cfh}>=$ Cairh

$\mathrm{Crh}=\mathrm{Cairh} / \mathrm{Cfh}$;

Cminh=Cairh;

else

$\mathrm{Crh}=\mathrm{Cfh} / \mathrm{Cairh}$;

$\mathrm{Cminh}=\mathrm{Cfh}$;

end

$\mathrm{Cfc}=\mathrm{cpwaterc} *$ mdot_amp;

if $\mathrm{Cfc}>=$ Cairc

$\mathrm{Crc}=\mathrm{Cairc} / \mathrm{Cfc}$;

Cminc=Cairc;

else

$\mathrm{Crc}=\mathrm{Cfc} / \mathrm{Cairc}$;

$\mathrm{Cminc}=\mathrm{Cfc}$;

end

qmaxh $=$ Cminh $*($ Tmixh-TH $) ; \% \mathrm{~W}$

qmaxc $=$ Cminc $*($ TC - Tmixc $) ; \% \mathrm{~W}$

$\mathrm{NTUc}=\mathrm{UAc} / \mathrm{Cminc}$;

$\mathrm{NTUh}=\mathrm{UAh} / \mathrm{Cminh}$;

effc $=1-\exp \left(1 / \mathrm{Crc}^{*} \mathrm{NTUc}^{\wedge} .22 *\left(\exp \left(-\mathrm{Crc} *(\mathrm{NTUc})^{\wedge} .78\right)-1\right)\right)$; 
$\operatorname{effh}=1-\exp \left(1 / \mathrm{Crh}^{*} \mathrm{NTUh}^{\wedge} .22 *\left(\exp \left(-\mathrm{Crh}^{*}(\mathrm{NTUh})^{\wedge} .78\right)-1\right)\right)$

$\mathrm{NTUvc}=\mathrm{NTUh}$;

effvc $=1-\exp (-\mathrm{NTUvc})$;

$\mathrm{Qc}=$ effc* qmaxc $\% \mathrm{~W}$

$\mathrm{Qh}=\mathrm{effh} * \mathrm{qmaxh} \% \mathrm{~W}$

Tairouth $=\mathrm{TH}+\mathrm{Qh} / \mathrm{Cairh}$;

Tairoutc $=$ TC-Qc/Cairc;

hhout=hmixh-Qh/(mdot_amp)*(Tmixh-TH)/abs(Tmixh-TH);

Thout=interp1(hhint, Tinth,hhout)

hcout $=$ hmixc $+Q c /($ mdot_amp $) *(T C-T m i x c) / a b s(T m i x c-T C)$;

Tcout=interp1(hcint,Tintc,hcout)

$\%$ call condensing hx model

[Qc_cond,Tcoutcond] $=\mathrm{hx} \_$cond2(UA,Aw,Ua,Aa,mdotairc,mdot_amp, TC,hain,Tmixc,ca, cpwaterc);

Tcoutcond

Tcout $=\max ($ Tcoutcond,Tcout $) ; \%$ use model result that ends up with most heat $x$ fer errorc $=$ Tcout $-\mathrm{TCR}$

errorh $=$ THR-Thout

if abs(Tcout-TCR) $<$ (ctol)

cdone $=1$;

end

if abs(THR-Thout) $<$ (htol)

hdone $=1$;

end

if cdone $==1 \&$ hdone $==1$

done $=1 ; \%$ relaxation complete

else \%update guess values

$\mathrm{THR}=\mathrm{THR}+\mathrm{wt}^{*}$ (Thout-THR);

$\mathrm{TCR}=\mathrm{TCR}+\mathrm{wt} *($ Tcout$-\mathrm{TCR})$;

end

input $=[$ mdot_amp L D $]$

count $=$ count +1

end

\section{Bed Correlations Function}

function[Nu,dP_ergun,keff] $=$ sph_part_1(Re,Pr,eps, kf, kr,v,dh,muf,rhof,cNu,cff,cnk);

$[\mathrm{n} \mathrm{m}]=\operatorname{size}(\mathrm{Re})$; alpha $=(1 \text {-eps })^{\wedge} 2 /$ eps $^{\wedge} 3$;

beta $=(1-$ eps $) /$ eps $^{\wedge} 3$;

$\mathrm{A}=180 ; \%$ for Ergun model from Fand et al

$\mathrm{B}=1.8 ; \%$ for Ergun model from Fand et al

for $\mathrm{j}=1: \mathrm{m}$

dP_ergun $(:, j)=\operatorname{cff}\left(A^{*}\right.$ alpha*muf(:,j)/dh^2*abs(v(j))+B*beta*rhof/dh*v(j)^2); \%Pa/m 
end

$\mathrm{Nu}=\mathrm{cNu} *(2+1.1 *$ Re.^(0.6).*Pr.^(1/3)); \%Wakao 1982

if eps $<0.0827$

$\mathrm{a} 0=10^{\wedge}(-4.898 * \mathrm{eps})$

elseif eps $<0.298$

$\mathrm{a} 0=10^{\wedge}\left(-.405-3.154^{*}(\right.$ eps-0.827) $)$;

elseif eps $<0.58$

$\mathrm{a} 0=10^{\wedge}(-1.084-6.778 *($ eps- 0.298$))$;

end

$\mathrm{f} 0=0.8+0.1 *$ eps;

$\mathrm{kcond}=\mathrm{kf}$ * $((1-\mathrm{a} 0) *(\mathrm{eps} * \mathrm{f} 0+\mathrm{kr} . / \mathrm{kf} *(1-\mathrm{eps} * \mathrm{f} 0)) . /(1-\mathrm{eps} *(1-\mathrm{f} 0)+\mathrm{kr} . / \mathrm{kf} * \mathrm{eps} *(1-$

$\mathrm{f} 0))+\mathrm{a} 0 *(2 *(\mathrm{kr} . / \mathrm{kf}) . \wedge 2 *(1-\mathrm{eps})+(1+2 * \mathrm{eps}) * \mathrm{kr} . / \mathrm{kf}) . /((2+\mathrm{eps}) * \mathrm{kr} . / \mathrm{kf}+1-\mathrm{eps})) ; \%$ Hadley

(1986) from Kaviany

kdisp $=.75 *$ eps*kf. Pr.*Re;

keff $=\mathrm{cnk}^{*}(\mathrm{kcond}+\mathrm{kdisp})$;

\section{Magnetic Field Function}

function $[\mathrm{muoH}]=$ muoHfunction_k2(x,t,tau,L,muoH_max,n_beds,L_ramp,arc_mag,L_ma g,magfunc);

$[\mathrm{n}, \mathrm{g}]=\operatorname{size}(\mathrm{x})$;

$[\mathrm{m}, \mathrm{g}]=\operatorname{size}(\mathrm{t})$;

if magfunc $==1$

\%spatially varying spinning regenerator

v_mag $=$ n_beds*L/tau; \%for bed A

$\% \quad$ v_mag $=-n \_$beds $*$ L/tau; \% for bed B

$\% \quad$ x_0_mag $=\left(\mathrm{L} \_\right.$mag- $0.75 *$ tau $*$ v_mag $) ; \%$ for bed B

x_0_mag $=-\left(\mathrm{L}+0 * \mathrm{~L} \_\mathrm{mag}-0.65 * \mathrm{tau}^{*} \mathrm{v} \_\mathrm{mag}\right) ; \%$ for bed A

x_mag_f $=$ v_mag*t+x_0_mag;

x_mag_b $=x \_m a g \_f-L \_m a g$;

x_mag_2_f $=$ x_mag_f-n_beds*L;

x_mag_2_b $=$ x_mag_b-n_beds*L;

for $\mathrm{i}=1: \mathrm{n}$

for $\mathrm{j}=1: \mathrm{m}$

if $\left(x(i)<=\left(x \_m a g \_f(j)\right)\right) \&\left(x(i)>=\left(x \_m a g \_f(j)-L \_r a m p\right)\right)$; $\operatorname{muoH}(i, j)=$ muoH_max* $\left(x \_m a g \_f(j)-x(i)\right) / L \_r a m p ;$

elseif $\left(x(i)<=\left(x \_m a g \_f(j)-L \_r a m p\right)\right) \&\left(x(i)>=\left(x \_m a g \_b(j)+L \_r a m p\right)\right)$; $\operatorname{muoH}(\mathrm{i}, \mathrm{j})=$ muoH_max;

$\operatorname{elseif}\left(x(i)<=\left(x \_m a g \_b(j)+L \_r a m p\right)\right) \&\left(x(i)>=\left(x \_m a g \_b(j)\right)\right)$; $\operatorname{muoH}(\mathrm{i}, \mathrm{j})=$ muoH_max $*\left(1-\left(\mathrm{x} \_\right.\right.$mag_b$(\mathrm{j})+\mathrm{L} \_$ramp-x(i))/L_ramp $)$;

elseif $\left(\left(x(\mathrm{i})<=\left(\mathrm{x} \_\right.\right.\right.$mag_2_f $\left.\left.(\mathrm{j})\right)\right) \&\left(\mathrm{x}(\mathrm{i})>=\left(\mathrm{x} \_\right.\right.$mag 2 _f $(\mathrm{j})-\mathrm{L} \_$ramp $\left.\left.)\right)\right)$ $\operatorname{muoH}(i, j)=$ muoH_max $*\left(x \_m a g \_2 \_f(j)-x(i)\right) / L \_r a m p$;

elseif(x(i)<=(x_mag_2_f(j)-L_ramp $)) \&\left(x(i)>=\left(x \_m a g \_2 \_b(j)+L \_r a m p\right)\right)$; muoH $(\mathrm{i}, \mathrm{j})=$ muoH_max; 


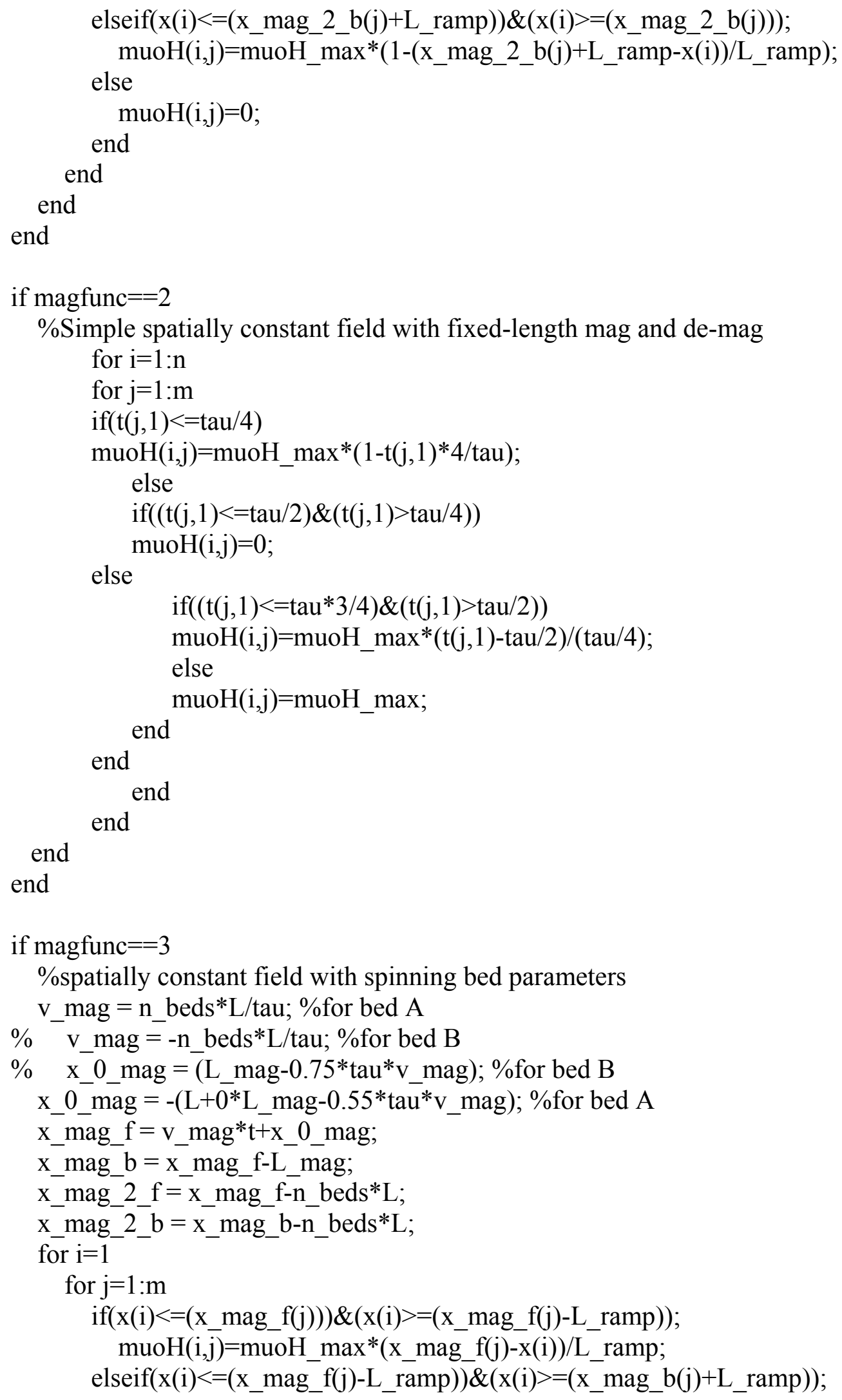




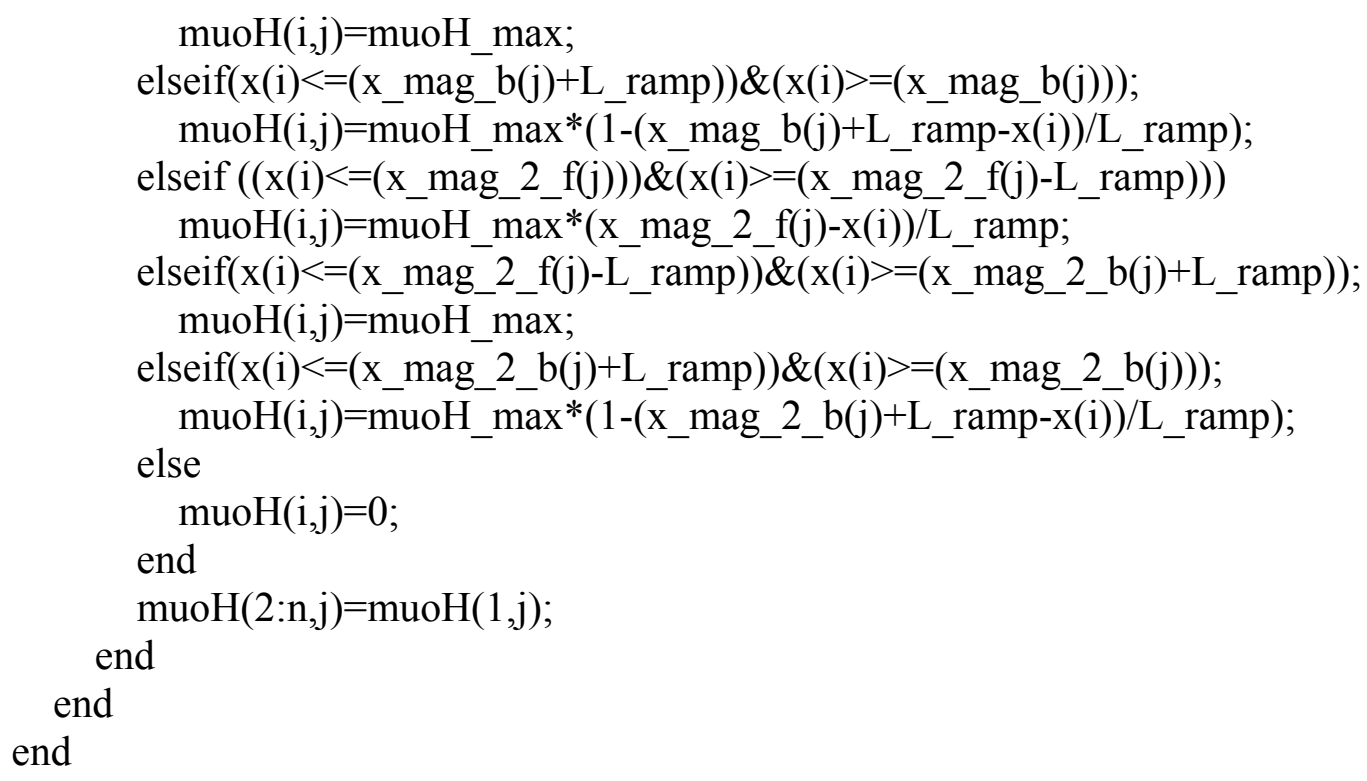

\section{Fluid Mass Flow Rate Function}

function[mdot] $=$ mdotfunction_k(t,tau,L,mdot_amp,Lf,n_bed);

mdot $=\operatorname{sign}(\operatorname{tau} / 2-\mathrm{t}) *$ mdot_amp; $\% \mathrm{~kg} / \mathrm{s}$

$[\mathrm{m}, \mathrm{g}]=\operatorname{size}(\mathrm{t})$;

set $=2$;

if $($ set $==1)$

$\%$ mass flow for $25 \%$ of the cycle

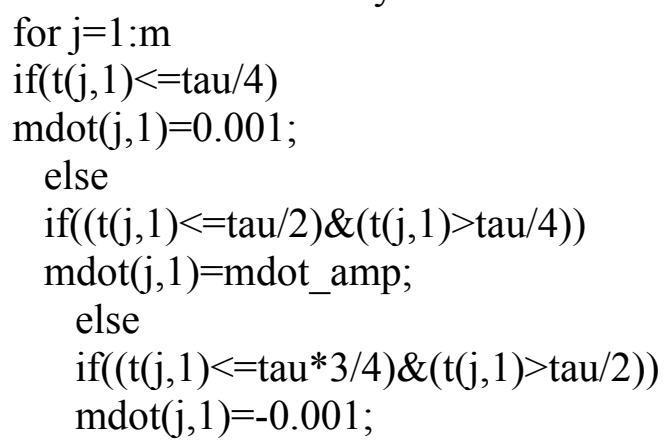




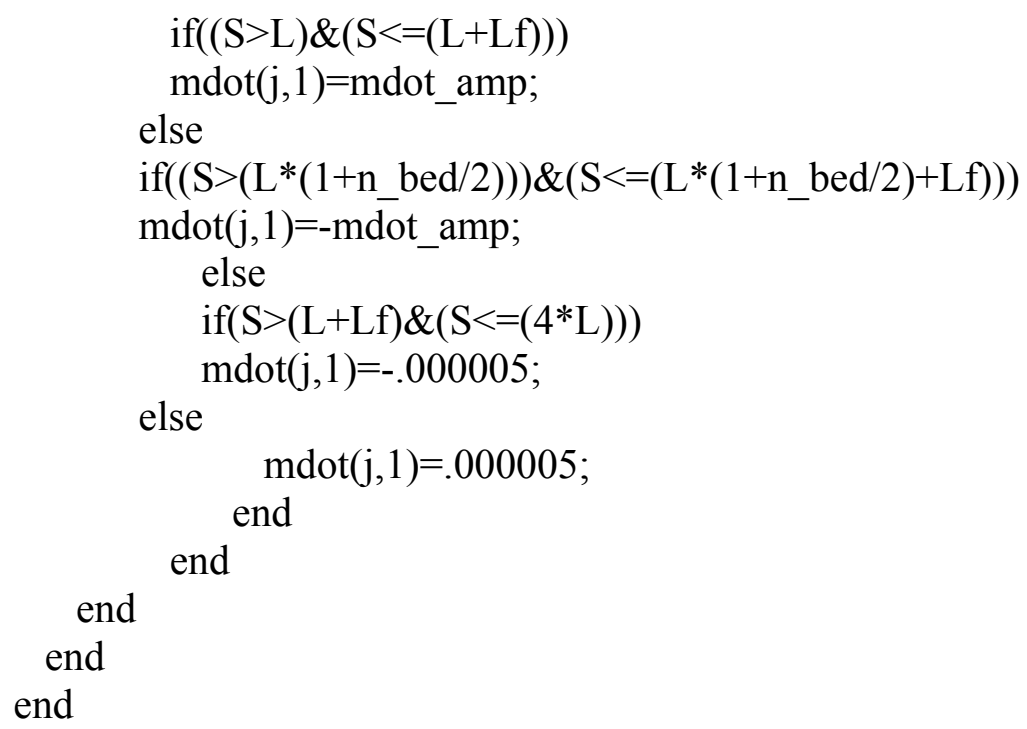


f_muf $=1.2 *[-0.605683313 .75601092-9.3240600211 .58832650-7.21611665$

1.80330228];

muf=polyval(f_muf,T_red); $\% \mathrm{~N}-\mathrm{s} / \mathrm{m}^{\wedge} 2$

$\mathrm{f}$ kf $=1.2 *[-0.7336194 \overline{3} 2.05522111-0.77427740]$;

kf=polyval(f_kf,T_red); \%W/m-K

f_cf $=1.2 *[-1288.185491 .12-7522.497526 .83]$;

f_Tcf=[-0.0000631041 $0.0735160042-27.52465536847526 .8319952701]$;

cf=polyval(f_cf,T_red); \% J/kg-K

cft=polyval(f_tcf,Tf); \%J/kg-K

cferrormax $=\max (\max (\operatorname{abs}(\mathrm{cf}-\mathrm{cft})))$;

rhof $=1.2 * 1000$;

int_cp=polyint(f_cf);

$\mathrm{hf}=($ polyval(int_cp,T_red)-polyval(int_cp, 1$))^{*} \mathrm{~T}$ _ref;

sf $=$ cf. $* \log ($ Tf./T_ref $)$;

\%\%\%\%\%\%\%\%\%\%\%\%\%\%\%\%\%\%\%\%\%\%\%\%\%\%\%\%\%\%

[n m] $=$ size(f_Tcf);

f_sf $=f \_T c f(1: m-1)$;

int_sf=polyint(f_sf);

$\mathrm{a}=\mathrm{f}-\mathrm{Tcf}(\mathrm{m})$;

sf_new $=$ (polyval(int_sf,Tf)-polyval(int_sf,T_ref) $)+\mathrm{a} * \log$ (Tf./T_ref);

\%\%\%\%\%\%\%\%\%\%\%\%\%\%\%\%\%\%\%\%\%\%\%\%\%\%\%\%\%\%\%\%

df_cf=polyder(f_Tcf);

dcf $=$ polyval(df_cf,Tf);

pcf=Tf./cf.*dcf; 